Gustavo Brattstroem Wagner

\title{
Análise Modal Operacional no Domínio do Tempo: Um Estudo Crítico dos Métodos de \\ Identificação
}

Dissertação de Mestrado

Dissertação apresentada como requisito parcial para obtenção do grau de Mestre pelo Programa de Pós-graduação em Engenharia Mecânica do Departamento de Engenharia Mecânica do Centro Técnico Científico da PUC-Rio.

Orientador : Prof. Rubens Sampaio Filho Coorientador: Profa. Roberta de Queiroz Lima 


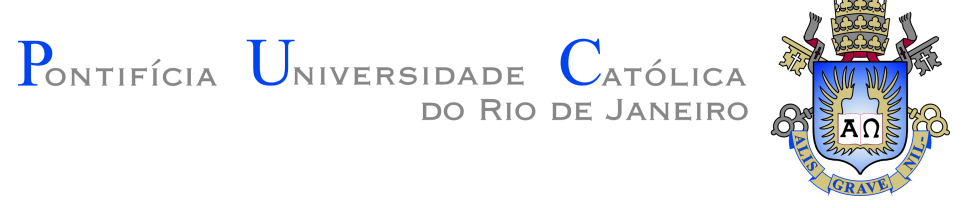

Gustavo Brattstroem Wagner

\begin{abstract}
Análise Modal Operacional no Domínio do Tempo: Um Estudo Crítico dos Métodos de Identificação
\end{abstract}

Dissertação apresentada como requisito parcial para obtenção do grau de Mestre pelo Programa de Pós-graduação em Engenharia Mecânica do Departamento de Engenharia Mecânica do Centro Técnico Científico da PUC-Rio. Aprovada pela Comissão Examinadora abaixo assinada.

Prof. Rubens Sampaio Filho

Orientador

Departamento de Engenharia Mecânica PUC-Rio

Profa. Roberta de Queiroz Lima

Coorientador

Departamento de Engenharia Mecânica PUC-Rio

Prof. Daniel Alves Castello

UFRJ

Prof. Thiago Gamboa Ritto

UFRJ

Prof. Márcio da Silveira Carvalho

Coordenador Setorial do Centro

Técnico Científico PUC-Rio

Rio de Janeiro, 22 de Agosto de 2017 
Todos os direitos reservados. É proibida a reprodução total ou parcial do trabalho sem autorização da universidade, do autor e do orientador.

\section{Gustavo Brattstroem Wagner}

Graduou-se em engenharia mecânica pela Pontifícia Universidade Católica do Rio de Janeiro.

Ficha Catalográfica

Wagner, Gustavo Brattstroem

Análise Modal Operacional no Domínio do Tempo: Um Estudo Crítico dos Métodos de Identificação/ Gustavo Brattstroem Wagner; orientador: Rubens Sampaio Filho; coorientador: Roberta de Queiroz Lima. - 2017.

v., 141 f: il. color. ; $30 \mathrm{~cm}$

Dissertação (mestrado) - Pontifícia Universidade Católica do Rio de Janeiro, Departamento de Engenharia Mecânica.

Inclui bibliografia

1. Engenharia Mecânica - Teses. 2. Análise Modal Operacional. 3. Identificação de Sistemas. 4. Vibrações Aleatórias. 5. Análise de Ruídos. 6. Testes Experimentais. I. Sampaio, Rubens. II. Lima, Roberta de Queiroz. III. Pontifícia Universidade Católica do Rio de Janeiro. Departamento de Engenharia Mecânica. IV. Título. 


\section{Agradecimentos}

Em primeiro lugar, gostaria de fazer um agradecimento especial ao meu orientador Prof. Rubens Sampaio pela oportunidade de desenvolver esta dissertação junto ao Laboratório de Vibrações. Gostaria de agradecer pela sugestão do tema de pesquisa, pelas inúmeras referências fornecidas, pela orientação e pelo exemplo de profissional.

Em segundo lugar, gostaria de agradecer a minha coorientadora Prof. Roberta Lima pelas várias discussões, críticas e ensinamentos nesses dois anos.

Ao Wagner, gostaria de agradecer pelo suporte no laboratório, pelo companheirismo e pela amizade construída durante esse mestrado.

Aos meus pais, gostaria de agradecer por todo apoio e carinho dado a mim durante toda a minha vida. Gostaria de agradecer por todos os sacrifícios feitos em prol do meu desenvolvimento pessoal e profissional.

Gostaria de agradecer também à minha namorada Juliana pela paciência durante os momentos mais difíceis e por me fazer ser sempre uma pessoa melhor.

Ao CNPq e à FAPERJ, eu gostaria de agradecer ao suporte financeiro durante meu mestrado. 


\section{Resumo}

Wagner, Gustavo Brattstroem; Sampaio, Rubens; Lima, Roberta de Queiroz. Análise Modal Operacional no Domínio do Tempo: Um Estudo Crítico dos Métodos de Identificação. Rio de Janeiro, 2017. 141p. Dissertação de Mestrado - Departamento de Engenharia Mecânica, Pontifícia Universidade Católica do Rio de Janeiro.

Análise modal consiste na caracterização de um sistema através dos seus parâmetros modais. Quando a principal excitação é causada pelo ambiente em que o sistema está inserido, essa caracterização é definida como análise modal operacional (OMA). Nestes casos, os forçamentos não são conhecidos (mensuráveis) e apenas as respostas são monitoradas. Por terem natureza aleatória, esses sinais precisam ser incorporados ao modelo numérico através de processos estocásticos. O principal objetivo desta dissertação consiste em descrever as técnicas de identificação em OMA. Para isso, duas vertentes foram criadas, uma teórica e outra experimental. Na parte teórica, as hipóteses necessárias para a identificação de um sistema por OMA são apresentadas. Uma análise dos erros causados por sinais ruidosos também é feita, permitindo que a sensibilidade dos métodos seja avaliada. Além de contemplar os principais métodos de identificação, dois novos métodos são propostos. Ambos foram desenvolvidos a partir da Decomposição Ortogonal Própria (POD) e combinam uma eficiência computacional com a possibilidade de quantificar as incertezas dos parâmetros. Na vertente experimental, o objetivo é ilustrar e validar a identificação de estruturas. Para isso, três diferentes bancadas foram criadas: um prédio de dois andares, uma pá eólica e uma ponte suspensa. Após a construção, essas estruturas foram devidamente instrumentadas por diferentes sensores. Um sistema de aquisição dados foi montado através de hardwares comerciais e analisados através de uma interfase gráfica desenvolvida especialmente para OMA pelo Laboratório de Vibrações.

\section{Palavras-chave}

Análise Modal Operacional; Identificação de Sistemas; Vibrações Aleatórias; Análise de Ruídos; Testes Experimentais; 


\section{Abstract}

Wagner, Gustavo Brattstroem; Sampaio, Rubens (Advisor); Lima, Roberta de Queiroz (Co-Advisor). Operational modal analysis in the time domain: a critical review of the identification methods. Rio de Janeiro, 2017. 141p. Dissertação de Mestrado

- Departamento de Engenharia Mecânica, Pontifícia Universidade Católica do Rio de Janeiro.

Modal analysis consists in the characterization of a system through its modal parameters. When the main excitation source is the system's environment, this characterization is defined as operational modal analysis (OMA). On those cases, the forces are unknown (not measured) and only the responses are monitored. Because of there random nature, those signals are incorporated into the numerical model as stochastic processes. The main goal of this dissertation is to describe the identification techniques in OMA. Therefore, two different approaches were created: a theoretical one and an experimental one. In the theoretical part, the required hypotheses for system's identification with OMA are presented. An analysis of the errors caused by noisy signals are also performed, allowing the method's sensibility to be evaluated. Besides the standard identification methods, two new ones are proposed. They both has been developed as extension of the Proper Orthogonal Decomposition (POD) and combine an efficient computational process with the possibility of quantify the parameters uncertainties. In the experimental approach, the goal is to illustrate and validate the identification of structures. Therefore, three different test bench were created: a two floor building, a wind turbine blade and a cable-stayed bridge. After their construction, those structures were proper instrumented with different sensors. A data acquisition system were built using commercial hardwares and analyzed through a graphic interface specially made for OMA and developed in the vibration laboratory.

\section{Keywords}

Operational Modal Analysis; System Identification; Random Vibration; Noise Analysis; Experimental Tests; 


\section{Sumário}

1 Introdução $\quad 13$

1.1 Aplicações de análise modal 13

$\begin{array}{lll}1.1 .1 & \text { Concepção do sistema } & 14\end{array}$

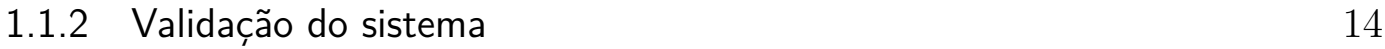

$\begin{array}{ll}\text { 1.1.3 Monitoramento do sistema } & 15\end{array}$

$\begin{array}{lll}1.2 & \text { Análise modal operacional } & 15\end{array}$

$\begin{array}{lll}1.3 & \text { Estrutura da dissertação } & 16\end{array}$

$\begin{array}{lll}1.4 & \text { Notação utilizada } & 18\end{array}$

2 Análise modal clássica $\quad 19$

2.1 Análise Modal de um sistema SDoF 20

2.1.1 Domínio do tempo 20

2.1.1.1 Resposta ao impulso unitário 21

2.1.1.2 Resposta a um forçamento generalizado 22

2.1.2 Domínio da frequência 24

2.1.2.1 Função de resposta em frequência 24

2.1.2.2 Propriedades e representações gráficas da FRF 26

2.1.3 Relação entre IRF e FRF 28

2.2 Análise Modal de um sistema MDoF 30

2.2.1 Resposta livre de um sistema MDoF sem amortecimento 31

2.2.2 Resposta de um sistema MDoF com amortecimento proporcional 35

2.2.3 Decomposição modal das FRFs e IRFs de um sistema MDoF com amortecimento viscoso proporcional $\quad 36$

2.2.4 Sistemas MDoF com amortecimento viscoso generalizado 39

3 Sinais aleatórios $\quad 47$

3.1 Função de correlação e densidade espectral de potência 48

3.2 Relação entre forçamento e resposta do sistema 49

3.3 Decomposição modal 51

3.3.1 Hipótese de forçamento por ruído branco 51

3.3.2 Hipótese de coordenadas modais não correlacionadas 58

4 Modelagens para a identificação de um sistema $\quad 62$

4.1 Modelo de espaço de estado $\quad 63$

4.1.1 Modelo contínuo 63

4.1.2 Modelo discreto 65

4.1.3 Parâmetros de Markov - Sistemas determinísticos 67

4.1.4 Parâmetros de Markov - Sistemas estocásticos 68

4.1.5 Domínio da frequência 69

$\begin{array}{ll}4.2 \text { Modelo ARMA } & 71\end{array}$

5 Influência do ruído nas funções de correlação $\quad 73$

5.1 Influência dos ruídos nas funções de correlação 73

5.1.1 Correlação da resposta do sistema com ruído 75 
5.1.2 Correlação da derivada da resposta do sistema com ruído

5.1.3 Correlação da integral da resposta do sistema com ruído 81

5.2 Conclusões 83

6 Métodos clássicos de identificação no domínio do tempo 85

6.1 Auto regressive / Poly Reference 86

6.2 Eigensystem Realization Algorithm 92

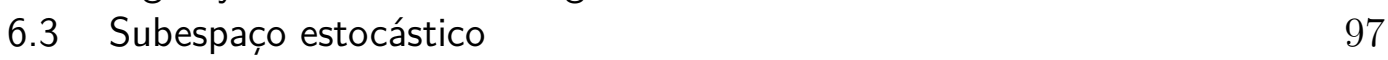

7 Novos métodos de identificação no domínio do tempo 108

$\begin{array}{lll}7.1 & \text { Smooth orthogonal decomposition } & 108\end{array}$

7.2 State-Variable Modal Decomposition 114

8 Conclusões $\quad 120$

$\begin{array}{ll}\text { Referências bibliográficas } & 123\end{array}$

$\begin{array}{lll}\text { A Sistema de aquisição de dados } & 130\end{array}$

$\begin{array}{lll}\text { B } & \text { Artigos produzidos } & 135\end{array}$ 


\section{Lista de figuras}

1.1 Interpretação de um sistema como um filtro 13

1.2 Hipótese sobre o forçamento em OMA 16

2.1 Representação de um forçamento como uma sequência de impulsos 23

2.2 Interpretação do sistema como um filtro no domínio do tempo. 24

2.3 Interpretação do sistema como um filtro no domínio da frequência. 26

2.4 Gráfico Bode de um sistema SDoF 27

2.5 Representação gráfica da parte real e imaginária da FRF 27

2.6 Gráfico de Nyquist 28

2.7 Exemplo de um sistem MDoF. 33

2.8 Decomposição modal da FRF de um sistema com amortecimento viscoso proporcional.

2.9 Decomposição modal da FRF de um sistema com amortecimento viscoso generalizado. $\quad 46$

3.1 Relação entre as estatísticas das forças e respostas do sistema. $\quad 47$

3.2 a) Realização do sinal $u(t)$. b) Estimativa da função de correlação.

c) Estimativa da densidade espectral de potência.

3.3 Exemplo da decomposição modal de uma PDS do sistema excitado por ruído branco.

3.4 Comparação entre a PDS da resposta do sistema calculada pelos parâmetros modais do sistema com a PSD calculada diretamente pelos sinais de resposta.

3.5 Exemplo da decomposição modal de uma PDS utilizando a hipótese de coordenadas modais não correlacionadas

5.1 Casos analisados

5.2 Respostas do sistema com adição de ruído

5.3 Correlações dos sinais: a) da resposta do sistema sem ruído, b) do ruído e c) da resposta do sistema com ruído

5.4 Distorção causada pelo ruído nas funções de correlação para diferentes proporções de ruído

5.5 Correlações do sinal após um processo de derivação: a) resposta do sistema sem ruído, b) ruído e c)resposta do sistema com ruído

5.6 Correlações do ruído após um processo de derivação com diferentes filtros

5.7 Correlações do sinal após o processo de integração: a)resposta do sistema sem ruído, b) ruído e c)resposta do sistema com ruído

5.8 Correlações do ruído após um processo de integração com diferentes filtros

6.1 Modelo de uma prédio de dois andares forçado pelo vento

6.2 Diagrama de estabilização do modelo do prédio utilizando o método de identificação AR/PR 
6.3 Formato dos modos de vibração do modelo do prédio identificados pelo método $A R / P R$

6.4 Bancada de uma ponte suspensa utilizada para ilustrar a identificação pelo método ERA

6.5 Diagrama de estabilização da bancada da ponte utilizando o método de identificação ERA

6.6 Formato dos modos de vibração da bancada da ponte identificados pelo método ERA

6.7 Pá eólica utilizada para ilustrar o método de identificação por subespaço estocástico

6.8 Posicionamento dos acelerômetros durante os quatro teste

6.9 Gráfico de convergência dos valores singulares de $\mathcal{H}$ utilizando diferentes números de colunas em $\mathcal{Y}_{p}$ e $\mathcal{Y}_{f}$

6.10 Diagramas de estabilização para os quatro testes feitos na pá eólica utilizando o método de identificação por subespaço estocástico

6.11 Formato dos modos de vibração da pá eólica identificados pelo método de subespaço estocástico

7.1 Ilustração do filtro passa banda que desliza sobre o espectro durante a identificação por SOD

7.2 Geometria e propriedades do modelo numérico de uma viga engatada utilizada para ilustrar a identificação por SOD

7.3 Diagrama de estabilidade obtida utilizando o filtro passa banda e o método de identificação SOD

7.4 Formato do modos de vibração identificados pelo método SOD 115

7.5 Sistema discreto de cinco graus de liberdade utilizado para ilustrar a identificação por SVMD

7.6 Exemplo de uma das funções de correlação obtidas na matriz $\mathbf{R}_{x x}\left(\tau_{k}\right) 118$

7.7 Histograma e envelope dos parâmetros modais identificados pelo método de SVMD

A.1 Diagrama de estabilidade obtida utilizando o filtro passa banda e o método de identificação SOD

A.2 Seleção dos parâmetros do teste no programa. 131

A.3 Adição dos sensores utilizados no teste. 131

A.4 Início da aquisição dos dados. 132

A.5 Gráfico das amostras obtidas no tempo. 132

A.6 Função de correlação das amostras coletadas. 133

A.7 Densidade espectral de potência das amostras coletadas. 133

A.8 Salvamento das amostras coletadas durante os testes. 134 


\section{Lista de tabelas}

2.1 Propriedades da transformada de Laplace 29

2.2 Parâmetros modais do sistema com amortecimento proporcional 39

2.3 Parâmetros modais do sistema com amortecimento viscoso generalizado

4.1 Comparação entre sistemas determinísticos e estocásticos $\quad 70$

6.1 Parâmetros modais identificados pelo método AR/PR 91

6.2 Parâmetros modais identificados pelo método ERA 97

6.3 Parâmetros modais identificados pelo método SSI - Teste 1

6.4 Parâmetros modais identificados pelo método SSI - Teste $2 \quad 104$

6.5 Parâmetros modais identificados pelo método SSI - Teste $3 \quad 106$

6.6 Parâmetros modais identificados pelo método SSI - Teste $4 \quad 106$

7.1 Frequências naturais da viga identificadas pelo método SOD 114

A.1 Equipamentos utilizados em cada um dos testes 130 


\section{Lista de Abreviaturas}
AR/PR - Auto regressive / Poly Reference
EMA - Análise modal experimental
ERA - Eigensystem realization algorithm
FRF - Função de resposta em frequência
IRF - Função de resposta ao impulso unitário
MAC - Modal assurance criterion
MDoF - Múltiplos graus de liberdade
OMA - Análise modal operacional
PSD - Densidade espectral de potência
SDoF - Um grau de liberdade
SOD - Smooth orthogonal decomposition
SSI - Identificação por subespaço estocástico
SVMD - State-variable modal decomposition 


\section{Introdução}

Para sistemas mecânicos, as relações entre os forçamentos aplicados e as respectivas respostas são definidas pelas leis de movimento. Quando um determinado sistema é modelado de forma linear e invariante no tempo, as relações entre essas duas grandezas são definidas de forma única. Por isso, sistemas desse tipo podem ser interpretado como filtros, onde sinais de força são processados e transformados em sinais de resposta, como mostra a Figura 1.1. A caracterização desses sistemas (filtros) pode ser realizada de diferentes formas. Um exemplo é pelos métodos de elementos finitos, onde estruturas contínuas são discretizadas e definidas através dos parâmetros de inércia, amortecimento e rigidez. Quando as frequências naturais, fatores de amortecimento e modos de vibração são utilizados, essa caracterização é chamada de análise modal [1].

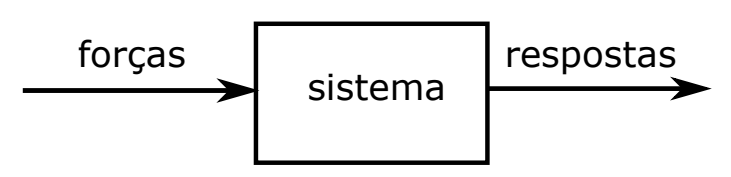

Figura 1.1: Interpretação de um sistema como um filtro

\section{1}

\section{Aplicações de análise modal}

Para diversos componentes mecânicos e estruturas, a análise modal pode ser aplicada em diferentes etapas: na concepção, na validação e durante operação. Em todas elas, a definição dos parâmetros modais é a principal tarefa, já que permite avaliar o bom funcionamento de um determinado sistema. A preocupação com os níveis de vibração e a confiabilidade de um componente estrutural tem se tornado cada vez mais importante nos dias de hoje. Por isso, a análise modal não tem sido apenas aplicada nas engenharias aeroespacial e mecânica, mas também na engenharia civil, nos problemas biomédicos, na acústica de instrumentos, em transporte e até mesmo em plantas nucleares [2]. 


\subsection{1}

\section{Concepção do sistema}

Quando componentes estruturais são projetados para atuar sob excitações dinâmicas, estudos sobre a sua eficiência, durabilidade e segurança precisam ser realizados. A análise modal, quando aplicada em modelos numéricos, permite estimar as frequências naturais e os modos de uma estrutura antes da sua construção. Dessa forma, alguns fenômenos indesejados, como a ressonância (caracterizado por um grande aumento nos níveis de vibração), podem ser evitados. Exemplos de estruturas em que isso acontece são pontes, aviões, rotores, etc. Em função das exigências imposta pelas normas, mercado e sociedade, a possibilidade de prever um bom funcionamento de um determinado componentes, ainda em sua etapa de concepção, resulta em um grande economia de tempo e recursos. Um bom estudo de caso é a ponte Millennium em Londres. Durante a sua inauguração em julho de 2000, constatou-se que a excitação causada pelo andar da pessoas fazia com que a mesma entrasse em ressonância. Por isso, a ponte precisou ser fechada até que esse problema fosse solucionado em 2002 com a adição de amortecedores [3].

\subsection{2}

\section{Validação do sistema}

Mesmo que um modelo numérico seja extremamente complexo e detalhado, ele continua sendo em uma representação matemática do sistema. Isso significa que algumas hipóteses ainda são realizadas e que podem divergir da realidade. Alguns exemplos de hipóteses na área de dinâmica de estruturas envolvem as propriedades dos materiais, condições de contorno, geometrias, etc. Por isso, após a construção de uma determinada estrutura, é geralmente necessário que os modelos numéricos sejam validados. Isso pode ser feito através da comparação entre os parâmetros modais obtidos numericamente e os identificados experimentalmente. Esse processo é chamado de teste modal e é a principal aplicação de análise modal $[4,5]$. Quando os parâmetros modais experimentais e numéricos divergem entre si, algumas técnicas para a atualização dos modelos precisam ser aplicadas $[6,7]$. Um exemplo desse procedimento ocorre na industria aeroespacial através do Ground Vibration Test (GVT). Nele, dado são coletados durante os testes de vibração no avião e utilizados para validar e atualizar seu modelo numérico. Essas informações são importantes para garantir a estabilidade aeroelástica da estrutura e definir os parâmetros para o Flutter Test [8]. 


\subsection{3}

\section{Monitoramento do sistema}

Uma outra aplicação da análise modal consiste no monitoramento da integridade estrutural (SHM) de um sistema [9, 10, 11, 12]. Neste caso, os parâmetros modais são quantificados periodicamente e comparados aos valores padrões (referentes ao sistema em perfeitas condições). Quando a diferença entre eles é significativa, há um indício de dano no sistema. Diferentes mecanismos são capazes de modificar dos parâmetros modais, sendo os principais deles: o aparecimento de trincas, a perda de torque nos parafusos, o excesso de corrosão, as modificações nas condições de contorno, etc. Através da alteração na massa ou da diminuição na rigidez, todos os parâmetros modais sofrem alteração. A localização onde o dano pode então ser definida pelos métodos de deteç̧ão que utilizam os parâmetros modais como fonte de informação. Exemplos onde o SHM tem sido aplicado nos últimos anos são as estruturas civis como pontes, passarelas e antigas construções [13, 14, 15, 16]. A grande preocupação nestes casos estão relacionados ao envelhecimento das estruturas, fazendo com que elas estejam próximo ao fim da vida útil determinada em projeto. Para que esse tempo seja prolongado, uma avaliação contínua da integridade estrutural tem sido realizada $[17,18]$.

\section{2}

\section{Análise modal operacional}

A análise modal operacional (OMA), tema desta dissertação, consiste na identificação de um sistema forçado pelo ambiente ou que está em condições operacionais. Essa técnica é indicada para as situações onde o forçamento desconhecido (não mensurável), causado pelo ambiente, é significativo e não pode ser eliminado (prazido para condições de laboratório). Esse tipo de excitação possuí uma natureza aleatória e precisa ser incorporado ao modelo do sistema na forma de processos estocásticos [19]

Para realizar a identificação de um sistema por OMA, algumas hipóteses são necessárias. A principal delas supõe que o forçamento aplicado é um processo estocástico cuja magnitude do espectro é diferente de zero para toda a banda de frequência analisada. Dessa forma, pode-se garantir que todos os modos existentes nessa banda serão excitados e consequentemente identificados. No desenvolvimento da teoria de OMA, uma hipótese ainda mais particular é geralmente feita: a do forçamento ser um ruído branco. A aplicação dessa hipótese é interessante pois permite um simplificação considerável na solução de alguma equações. Mesmo que esse tipo de forçamento não possa ser obtido na prática, ele continua sendo aplicável. Isso porque um filtro 
pode ser sempre utilizado na transformação de um ruído branco em um outro "colorido"(mais compatível com a realizada). A Figura 1.2 ilustra essa ideia. Quando a identificação do sistema é realizada utilizando essa hipótese, tanto os parâmetros relacionados ao filtro de forçamento quando os relacionados ao sistema físico são obtidos. A distinção entre eles só pode ser realizada pelo analista ao avaliar os respectivos resultados. Por exemplo, quando o fator de amortecimento é extremamente alto ou negativo, supõe-se que o respectivo parâmetro (polo) é vinculado ao filtro de forçamento, já que os valores típicos para o amortecimento em estruturas variam entre 0.5 e $3 \%$. Uma outra forma de classificar os parâmetros é através do formato dos modos identificados. Caso o modo tenha um formato realista (coerente com uma previsão numérica ou teórica), os parâmetros são relacionados ao sistema físico. Coso o contrário, são relacionados ao filtro de forçamento.

sistema identificado por OMA

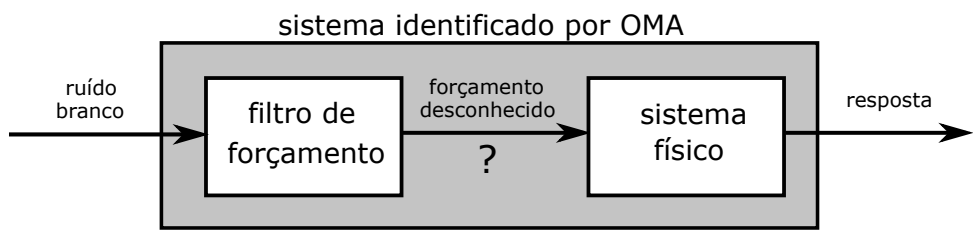

Figura 1.2: Hipótese sobre o forçamento em OMA

Ao ser comparada com a análise modal experimental (EMA) [20], onde a identificação é feita através do sinais de força e resposta, a análise modal operacional contém vantagens e desvantagens. As principais vantagens estão relacionadas à identificação de estruturas que não podem ser trazidas para laboratórios, como por exemplo, prédios, pontes, turbinas eólicas, satélites em orbita, etc. A aplicação de EMA nessas estruturas seriam altamente custosa e demorada, além de não garantir bons resultados devido à excitação do ambiente não considerada. Já as desvantagens estão relacionadas a precisão dos resultados. Por não poder garantir as características da excitação, os resultados podem muitas vezes não serem ideais. Além disso, os métodos de identificação são muitas vezes mais sofisticados e de difícil compreensão, já que envolvem conceitos estatísticos nos modelos e no tratamento dos forçamentos desconhecidos [21].

\section{3}

\section{Estrutura da dissertação}

Para que a identificação de sistemas utilizando OMA seja bem feita, uma base teórica precisa ser primeiro apresentada. No capítulo 2, a caracterização de sistemas lineares excitados por forças determinísticas é feita. Para isso, as funções de resposta ao impulso e as funções de resposta em frequência são 
definidas. Ambas são importantes porque possibilitam relacionar os sinais de força com os de resposta, além de poderem ser decompostas em relação ao parâmetros modais.

No capítulo 3, estuda-se sistemas lineares excitados por forças aleatórias. Neste caso, é preciso que as relações entre algumas estatísticas (correlações e densidades espectrais) das forças e das respostas do sistema sejam definidas. Além disso, algumas hipóteses necessárias em OMA sobre o sistema e o forçamento são apresentadas. Através dessas hipóteses é mostrado que as funções de correlação e de densidade espectral de potência das respostas de um sistema podem ser decompostas em relação aos parâmetros modais.

No capítulo 4 são apresentados alguns modelos do sistema que passaram por um processo de discretização em relação ao tempo. Esses modelos são importantes do ponto de vista experimental, já que permitem utilizar diretamente os sinais coletados durante os testes (digitais). Além disso, mostra-se como os parâmetros modais podem ser extraídos desses modelos.

Quando a análise modal operacional é realizada no domínio do tempo, as principais funções utilizadas para extrair informações sobre o sistema são as funções de correlação. Por isso, o capítulo 5 apresenta uma análise não encontrada na literatura sobre a distorção dessas funções quando estimada por sinais ruidosos. Isso permite entender como alguns métodos de identificação são mais sensíveis em relação ao ruído que outros.

O capítulo 6 apresenta alguns dos métodos de identificação no domínio do tempo mais populares em OMA. Ao fim de cada método, um exemplo experimental da identificação de uma estrutura é mostrado. Esses exemplos servem para validar a implementação dos métodos e mostrar um procedimento para a análise dos resultados.

O capítulo 7 contém a principal contribuição desta dissertação. Duas novas implementações são propostas para dois diferentes métodos. Os métodos foram desenvolvidos a partir da Decomposição Ortogonal Própria (POD) e por ainda estarem em desenvolvimento apresentam algumas limitações. As duas novas implementações permitem uma melhora no desempenho dos métodos em relação a sensibilidade do ruído e em relação a quantidade de modos identificados. Exemplos numéricos foram utilizados para ilustrar os métodos e as novas implementações.

O oitavo e último capítulo desta dissertação trata das principais conclusões feitas neste trabalho e discute possíveis trabalhos futuros.

Esta dissertação contém também dois anexos. O primeiro apresenta os equipamentos utilizados durante os testes experimentais, assim como um manual para a utilização da interface gráfica desenvolvida no Laboratório de 
Vibrações para a aquisição de dados em OMA. O segundo anexo apresenta a capa os artigos produzidos durante esse mestrado.

\section{4}

\section{Notação utilizada}

Durante esta dissertação, procurou-se ao máximo manter uma notação coerente em relação a dimensão dos parâmetros e funções. Quando escalares, utilizou-se letras minúsculas. Para vetores, utilizou-se letras minúsculas em negrito. Já para as matrizes, utilizou-se letras maiúsculas e em negrito.

Para as funções, quando definidas fora do domínio do tempo (Laplace ou da frequência), utilizou-se o acento til no topo das letras. Quando funções ou parâmetros foram estimados, o acento circunflexo foi utilizado. 


\section{2}

\section{Análise modal clássica}

No estudo da dinâmica, o principal objetivo consiste em descrever, ao longo do tempo, as respostas de um certo sistema em função dos forçamentos aplicados e condições iniciais. Já em análise modal, o objetivo passa a ser caracterizar esse sistema através dos seus parâmetros modais, isto é, através das suas frequências naturais, fatores de amortecimento e os modos de vibração. Pelo fato destes parâmetros serem ditos globais (dependerem do sistema como um todo), esse tipo de análise permite uma definição mais simples do sistema, já que necessita menos parâmetros para caracteriza-lo. Por exemplo, quando os sistemas são modelados pelo método de elementos finitos, em geral, um grande número de parâmetros de massa e rigidez são necessários. Assim, a identificação experimental destes parâmetros seria um tarefa difícil e trabalhosa. Já em análise modal, apenas poucos parâmetros são necessários para caracterizar esse mesmo sistema com precisão equivalente. Por isso, a identificação experimental de um sistema é realizada na maioria dos casos em função dos parâmetros modais.

Uma forma de interpretar um sistema consiste em analisá-lo como um filtro, onde os sinais de entrada (forçamentos) são processados e transformados em sinais de saída (deslocamentos, velocidades e acelerações). As relações entre esses sinais são estabelecidas através das chamadas funções de resposta ao impulso (IRF) e das funções de resposta em frequência (FRF). Diferentemente do deslocamento, velocidade ou aceleração do sistema ao longo do tempo, essas funções são independentes do forçamento e por isso são utilizadas na caracterização o sistema. A teoria por trás da análise modal consiste em decompor as IRF e as FRF em relação aos parâmetros modais. Dessa forma, esses parâmetros podem ser identificados experimentalmente através de métodos de ajustes de curvas nos dados coletados.

Este capítulo é dividido em duas partes. Na primeira, a análise modal de um sistema de um grau de liberdade (SDoF) é discutida. Este tipo de sistema, apesar de simples, é extremamente importante do ponto de vista teórico. Seus conceitos podem ser facilmente compreendidos e seus respectivos resultados são estendidos e utilizados em análise de sistemas mais complexos. Na segunda etapa, sistemas com múltiplos graus de liberdade (MDoF) passam 
a ser analisados. Este tipo de sistema, apesar de mais complexo, pode ser desacoplado e transformado em diversos sistemas SDOF, cujas soluções são simples e diretas. Neste capítulo, o objetivo é definir as suas respectivas IRF e FRF em função dos parâmetros modais. Três tipos de modelos para o amortecimento dos sistemas são tratados: sem amortecimento (sistemas conservativos), com amortecimento viscoso proporcional e com amortecimento viscoso generalizado. Outros mecanismos de dissipação, com por exemplo o amortecimento estrutural, podem ser encontrados em [2] e [22].

\section{1}

\section{Análise Modal de um sistema SDoF}

Nesta seção será feita a análise modal de um sistema SDoF tanto no domínio do tempo quanto no domínio da frequência. A relação existente entre elas também será apresentada. Um sistema SDoF (single degree of freedom) é todo sistema cuja dinâmica pode ser bem representada com apenas uma coordenada. Quando o sistema é linear e composto por um parâmetro de massa $m$, rigidez $k$ e amortecimento $c$, sua dinâmica é dada por

$$
m \ddot{q}(t)+c \dot{q}(t)+k q(t)=u(t) .
$$

A função $q(t)$ representa o deslocamento da massa e $u(t)$ o forçamento nela aplicado. Nesta dissertação só serão tratado sistemas lineares invariantes no tempo, ou seja, sistemas com coeficientes constantes.

\subsection{1}

\section{Domínio do tempo}

No domínio do tempo, a função de resposta ao impulso unitário (IRF) desempenha um papel fundamental na caracterização de um sistema. Uma excitação do tipo impulso consiste na aplicação de um forçamento de forma instantânea e pontual. Como visto a seguir, esse tipo de forçamento resulta em oscilações livres do sistema que podem ser escritas em função dos parâmetros modais. A partir da IRF é possível obter a resposta do sistema para qualquer forçamento. Isso porque um forçamento generalizado pode ser sempre interpretado como uma sequência de impulsos.

Ao supor que os sistemas são lineares, a hipótese de superposição das respostas pode ser aplicada [23]. Essa hipótese afirma que, se $q_{1}(t)$ é a resposta do sistema com forçamento $u_{1}(t)$ e $q_{2}(t)$ é a resposta do sistema com forçamento $u_{2}(t)$, então $q(t)=q_{1}(t)+q_{2}(t)$ é a resposta do sistema para um forçamento $u(t)=u_{1}(t)+u_{2}(t)$. O somatório das respostas de cada impulso pode ser realizada através do operador de convolução, definido mais a frente. 


\subsubsection{1}

\section{Resposta ao impulso unitário}

Uma das formas de se representar a função impulso unitário é dado pela equação

$$
\delta(t)=\lim _{\Delta t \rightarrow 0}\left\{\begin{array}{ll}
\frac{1}{\Delta t}, & t \leq\left|\frac{\Delta t}{2}\right| \\
0, & t>\left|\frac{\Delta t}{2}\right|
\end{array}=\left\{\begin{array}{ll}
+\infty, & t=0 \\
0, & t \neq 0
\end{array} .\right.\right.
$$

Isso significa que a função impulso unitário é nula em qualquer instante de tempo exceto em $t=0$, onde a função passa a ser infinito. O impulso é dito unitário quando sua integral em todo o domínio do tempo é igual a um, isto é,

$$
\int_{-\infty}^{+\infty} \delta(t) d t=1
$$

Ao adotar a lei de conservação de movimento para um sistema massa-modaamortecedor em um instante de tempo imediatamente antes do impulso, $t=0^{-}$, e outro imediatamente após, $t=0^{+}$, obtêm-se a seguinte relação

$$
\int_{0^{-}}^{0^{+}} \delta(t) d t=1=m \dot{q}\left(0^{+}\right)-m \dot{q}\left(0^{-}\right) .
$$

Supondo que o sistema esteja inicialmente em repouso, tem-se que $\dot{q}\left(0^{-}\right)=$ 0. Zerando este termo na Eq. (2-4), conclui-se que o impacto causa uma descontinuidade na velocidade, uma vez que em $t=0^{+}$a velocidade passa a ser

$$
\dot{q}\left(0^{+}\right)=\frac{1}{m} .
$$

Dessa forma, a resposta ao impulso unitário será equivalente à resposta do sistema livre (solução homogenia da Eq.(2-1)) com condições iniciais $q(0)=0$ e $\dot{q}(0)=1 / m$. A dinâmica do sistema é então equivalente a

$$
\begin{array}{r}
m \ddot{q}(t)+c \dot{q}(t)+k q(t)=0 \\
\text { cond. iniciais }=\left\{\begin{array}{l}
q(0)=0 \\
\dot{q}(0)=\frac{1}{m}
\end{array} .\right.
\end{array}
$$

Essa equação pode ser reescrita em função dos parâmetros modais do sistema ao dividir tudo por $m$, resultando em

$$
\ddot{q}(t)+2 \zeta \omega_{n} \dot{q}(t)+\omega_{n}^{2} q(t)=0,
$$

onde, $\zeta=\frac{c}{2 m \omega_{n}}$ é o fator de amortecimento e $\omega_{n}=\sqrt{\frac{k}{m}}$ a frequência natural do sistema conservativo associado. Para a solução da Eq. (2-6) ou Eq. (2-7), propõe-se uma função exponencial na forma

$$
q(t)=e^{\lambda t}
$$

Calculando a $1^{\mathrm{a}}$ e a $2^{\mathrm{a}}$ derivada da solução proposta e substituindo os respectivos resultado na equação da dinâmica Eq. (2-7), obtêm-se: 


$$
\left(\lambda^{2}+2 \zeta \omega_{n} \lambda+\omega_{n}^{2} \omega\right) e^{\lambda t}=0 .
$$

Considerando que $e^{\lambda t} \neq 0$, o polinômio característico do sistema, escrito em função dos parâmetros modais, é obtido:

$$
\lambda^{2}+2 \zeta \omega_{n} \lambda+\omega_{n}^{2} \omega=0
$$

As soluções $\lambda$ desse polinômio são chamadas de polos do sistema, e, para um sistema sub-amortecido $(\zeta<1)$, formam o par de complexos conjugados

$$
\left\{\begin{array}{l}
\lambda=-\zeta \omega_{n}+i \omega_{d} \\
\lambda^{*}=-\zeta \omega_{n}-i \omega_{d}
\end{array},\right.
$$

onde o termo $\omega_{d}=\omega_{n} \sqrt{1-\zeta^{2}}$ é chamado de frequência natural amortecida do sistema. Substituindo a Eq. (2-11) na Eq. (2-9) obtêm-se a solução livre do sistema na forma

$$
q(t)=A e^{\lambda t}+B e^{\lambda^{*} t}=e^{-\zeta \omega_{n} t}\left(A \cos \omega_{d} t+B \sin \omega_{d} t\right),
$$

onde $A$ e $B$ são as constantes a serem definidas pelas condições iniciais.

A resposta ao impulso unitário, definida como $h$, é obtida impondo as condições iniciais $q(0)=0$ e $\dot{q}(0)=1 / m$ em (2-12), resultando em $A=0$ e $B=\frac{1}{m \omega_{d}}[24]$. Assim, a resposta ao impulso unitário de um sistema SDoF com amortecimento viscoso é dado pela equação

$$
h(t)=\frac{e^{-\zeta \omega_{n} t}}{m \omega_{d}} \sin \omega_{d} t .
$$

Uma importante característica dessa função é o fato dela ser definida utilizando apenas os parâmetros modais. Por isso, ela pode ser aplicada para caracterizar e identificar o sistema.

\subsubsection{2}

\section{Resposta a um forçamento generalizado}

A resposta ao impulso unitário pode ser usada para o calculo da resposta do sistema à qualquer forçamento. Para isso, idealiza-se o forçamento como uma superposição de impulsos, transladados no tempo e com diferentes magnitudes. Essa idealização é mostrada na Figura 2.1. Devido a linearidade do sistema, a resposta será dada pela superposição das respostas aos impulsos. Dessa forma, calcula-se a resposta a cada um dos impulsos individualmente e em seguida os resultados são somados. Esse processo é realizado pelo operador de convolução mostrado a seguir.

O cálculo da resposta do sistema para o impulso em um instante de tempo $t_{i}$ é aproximadamente 


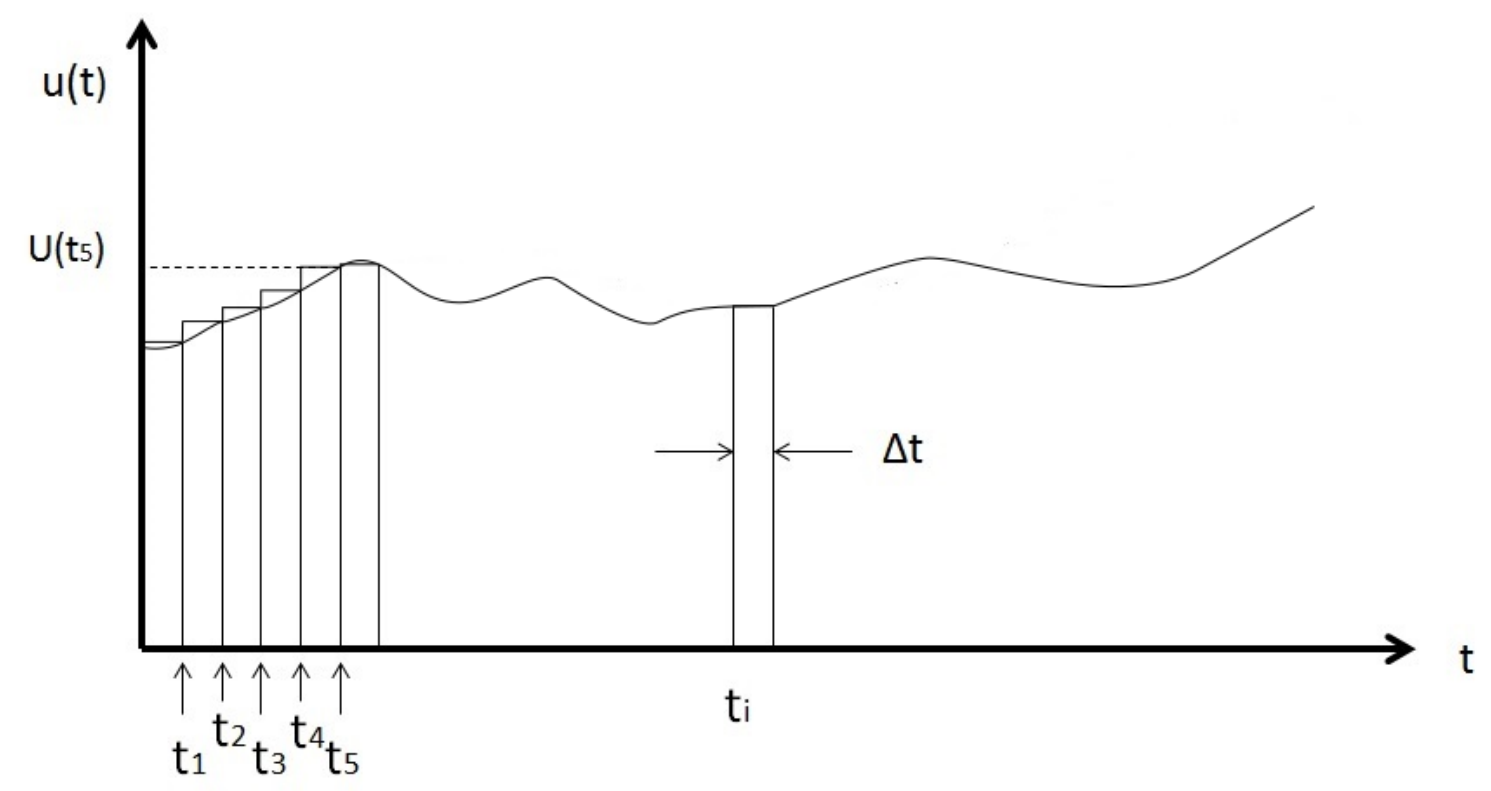

Figura 2.1: Representação de um forçamento como uma sequência de impulsos

$$
q_{i}(t) \approx\left[u\left(t_{i}\right) \Delta t\right] h\left(t-t_{i}\right)
$$

O termo $u\left(t_{i}\right) \Delta t$ representa a magnitude do impulso, visto que o mesmo não é mais necessariamente unitário. Essa magnitude é aproximada como a área dos retângulos formados na Figura 2.1. O termo $h\left(t-t_{i}\right)$ é a função definida na Eq. (2-2), no entanto transladado no tempo para o instante $t_{i}$.

Utilizando o princípio da superposição, a solução do forçamento generalizado é definido como a soma das respostas de cada um dos impulsos. Assim, a resposta final do sistema após $j$ intervalos de tempo é dado por

$$
q(t)=\sum_{i=1}^{j} q_{i}(t) \approx \sum_{i=1}^{j} u\left(t_{i}\right) h\left(t-t_{i}\right) \Delta t .
$$

Pela definição de impulso, o intervalo de tempo $\Delta t$ tende a zero. O somatório na Eq. (2-15) se torna então uma integral, e resulta em

$$
q(t)=\int_{0}^{t} u(\theta) h(t-\theta) d \theta=u(t) * h(t)
$$

Na equação acima, supõe-se que o forçamento seja nulo para $t<0$. A Eq. (2-16) é a definição de convolução entre o sinal do forçamento e a resposta ao impulso unitário de um sistema causal ${ }^{1}(h(t)=0$ para $t<0)$. Através dessa equação, pode-se observar como o sistema comporta-se como um filtro, processando um sinal de forçamento (entrada) em um de deslocamento (saída). Essa interpretação é esquematizada pela Figura 2.2.

\footnotetext{
${ }^{1}$ Em um sistema causal, os forçamentos futuros não influenciam o estado presente
} 


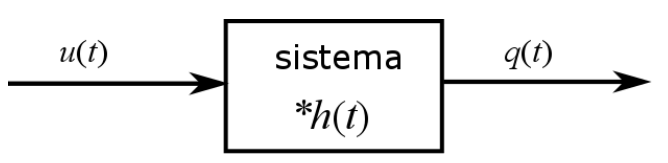

Figura 2.2: Interpretação do sistema como um filtro no domínio do tempo.

\subsection{2}

\section{Domínio da frequência}

No domínio da frequência, deseja-se avaliar o comportamento do sistema quando excitado por forçamentos harmônicos. Neste estudo existem duas características a serem avaliadas: a magnitude das oscilações e fase entre os sinais de forçamento e resposta do sistema. Essas informações são obtidas pela chamada função de resposta em frequência (FRF). Através dela é possível visualizar com mais facilidade as principais características modais do sistema.

\subsubsection{1}

\section{Função de resposta em frequência}

Seja $u(t)$ um forçamento harmônico na forma $u(t)=\tilde{u}(\omega) e^{i \omega t}$, onde $\tilde{u}(\omega)$ é uma função real que representa a amplitude desse forçamento em relação à frequência de forçamento $\omega$. Supondo que a resposta do sistema também seja harmônica e com a mesma frequência de forçamento, tem-se que $q(t)=\tilde{q}(\omega) e^{i \omega t}$. A função $\tilde{q}(\omega)$ é complexa e define a amplitude do movimento e a respectiva fase entre o forçamento e a resposta [2]. Substituindo $u(t)$ e $q(t)$ (após realizar as devidas derivadas) na Eq. (2-1) obtêm-se como resultado

$$
\underbrace{\left(-m \omega^{2}+c \omega i+k\right)}_{k_{\text {din }}} \tilde{q}(\omega) e^{i \omega t}=\tilde{u}(\omega) e^{i \omega t} .
$$

O termo $k_{d i n}$ é chamado de rigidez dinâmica do sistema e pode ser reescrito também em função dos parâmetros modais (frequência natural e fator de amortecimento)

$$
\begin{aligned}
k_{d i n} & =k\left[1-\left(\frac{\omega}{\omega_{n}}\right)^{2}+2 i \zeta\left(\frac{\omega}{\omega_{n}}\right)\right] \\
& =k\left(1-r^{2}+2 i \zeta r\right),
\end{aligned}
$$

onde $r=\frac{\omega}{\omega_{n}}$. Ao reescrever a Eq. (2-17) como a razão entre as amplitudes de deslocamento e de forçamento, obtêm-se

$$
\tilde{h}(\omega)=\frac{\tilde{q}(\omega)}{\tilde{u}(\omega)}=\frac{1}{k_{d i n}} .
$$


Existem diferentes formas de representar a função $\tilde{h}(\omega)$. Dentre elas, as mais populares são

$$
\tilde{h}(\omega)=\frac{1}{k-m \omega^{2}+i c \omega}=\frac{1}{k\left(1-r^{2}+2 i \zeta r\right)}=\frac{1}{m(i \omega-\lambda)\left(i \omega-\lambda^{*}\right)} .
$$

As constantes $\lambda$ e $\lambda^{*}$ na última igualdade são os mesmos polos do sistema definidos pela Eq. (2-11). A função $\tilde{h}(\omega)$ é chamada de função de resposta em frequência (FRF) do deslocamento de um sistema SDoF com amortecimento viscoso. Percebe-se que a função $\tilde{h}(\omega)$ na Eq. (2-20) é complexa e definida apenas pelos parâmetros do sistema, isto é, independente do forçamento. Para o caso particular em que o amortecimento é nulo, $\tilde{h}(\omega)$ se transforma em uma função real. A última igualdade na Eq. (2-20) pode ainda ser reescrita de forma fatorada, também chamada de forma residual, conforme a equação

$$
\tilde{h}(\omega)=\frac{R}{i \omega-\lambda}+\frac{R^{*}}{i \omega-\lambda^{*}},
$$

onde

$$
R=\frac{1}{2 m \omega_{d} i} .
$$

A constante $R$ é chamada de coeficiente residual ${ }^{1}$.

A FRF definida pela Eq. (2-20) permite avaliar as principais características do sistema sob forçamentos harmônicos: magnitude das oscilações e fase entre o deslocamento e o forçamento. Utilizando as propriedades dos números complexos, a magnitude e fase de $\tilde{h}(\omega)$ podem ser obtidos através das equações

$$
\begin{gathered}
|\tilde{h}(\omega)|=\frac{k}{\sqrt{\left(k-m \omega^{2}\right)^{2}+c^{2} \omega^{2}}}=\frac{1}{\sqrt{\left(1-r^{2}\right)^{2}+(2 \zeta r)^{2}}} \\
\angle \tilde{h}(\omega)=\tan ^{-1}\left[\frac{-c \omega}{k-m \omega^{2}}\right]=\tan ^{-1}\left[\frac{-2 \zeta r}{1-r^{2}}\right] .
\end{gathered}
$$

Seguindo o mesmo procedimento realizado para o deslocamento, a FRF do sistema em relação a sua velocidade e aceleração pode ser facilmente obtida. Para isso, basta perceber que a velocidade é expressa por $\dot{q}(t)=i \omega \tilde{q}(\omega) e^{i \omega t}$. Realizando a mesma operação para a aceleração (segunda derivada), as seguintes FRF são obtidas:

$$
\begin{array}{ll}
\text { Velocidade } & \Rightarrow \quad \tilde{h}_{v}(\omega)=\frac{i \omega}{k-m \omega^{2}+i c \omega}=\frac{i \omega}{k\left(1-r^{2}+2 i \zeta r\right)} \\
\text { Aceleração } \quad \Rightarrow \quad \tilde{h}_{a}(\omega)=\frac{-\omega^{2}}{k-m \omega^{2}+i c \omega}=\frac{-\omega^{2}}{k\left(1-r^{2}+2 i \zeta r\right)} .
\end{array}
$$

Ao utilizar as FRFs, pode-se utlizar mais uma vez a analogia de um filtro para os sistemas, como mostra a figura 2.3. Através da Eq. (2-19), o sinal da resposta do sistema no domínio da frequência pode ser obtido através do

\footnotetext{
${ }^{1}$ Apesar de definido por uma letra maiúscula, esse termo é um escalar
} 
produto entre o sinal de entrada no domínio da frequência e a FRF

$$
\tilde{q}(\omega)=\tilde{h}(\omega) \tilde{u}(\omega)
$$

Diferentemente da relação obtida no domínio do tempo, onde o operador de convolução é necessário, no domínio da frequência utiliza-se apenas uma multiplicação. Por isso, do ponto de vista computacional, o domínio da frequência é mais atraente.

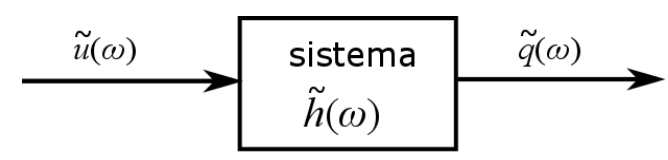

Figura 2.3: Interpretação do sistema como um filtro no domínio da frequência.

\subsubsection{2}

\section{Propriedades e representações gráficas da FRF}

As FRFs definidas pelas Eqs. (2-19/2-24/2-25) podem ser representadas graficamente de diferentes formas, onde cada uma delas evidencia de forma mais clara certa característica. A seguir, as representações mais comuns das FRFs são mostradas, assim como suas respectivas propriedades.

\section{Gráfico Bode}

O conjunto dos gráficos da magnitude e o da fase é chamado de gráfico Bode. Esta representação é a mais comum em análise modal, uma vez que ela ilustra de forma clara as características dos parâmetros modais.

Na Figura 2.4, o gráfico Bode de um sistema SDoF é mostrado de forma adimensional. Diferentes valores para o amortecimento são utilizados para mostrar a influência desses parâmetros na resposta do sistema. Na magnitude, percebe-se a existência de um pico nas proximidades de $r=1$. Esse fenômeno é chamado de ressonância, e ocorre quando a frequência de forçamento tem valor próximo ao da frequência natural do sistema. A ressonância é caracterizada pelo grande aumento da amplitude na resposta e uma mudança de fase. Percebe-se pelos gráficos que, na ressonância, a fase entre a resposta e o forçamento é de $\pi / 2$ rad. Para o sistema sem amortecimento, a mudança de fase ocorre de forma abrupta indo de 0 a $\pi$, além da sua magnitude tender a infinito. Já quando o amortecimento é alto, o ponto máximo da curva fica ligeiramente deslocado para a esquerda da frequência natural. $\mathrm{O}$ amortecimento da estrutura pode ser qualitativamente analisado através da relação entre a espessura e a altura dos picos. Percebe-se que quanto menor for o amortecimento, mais esbelto é o pico. 

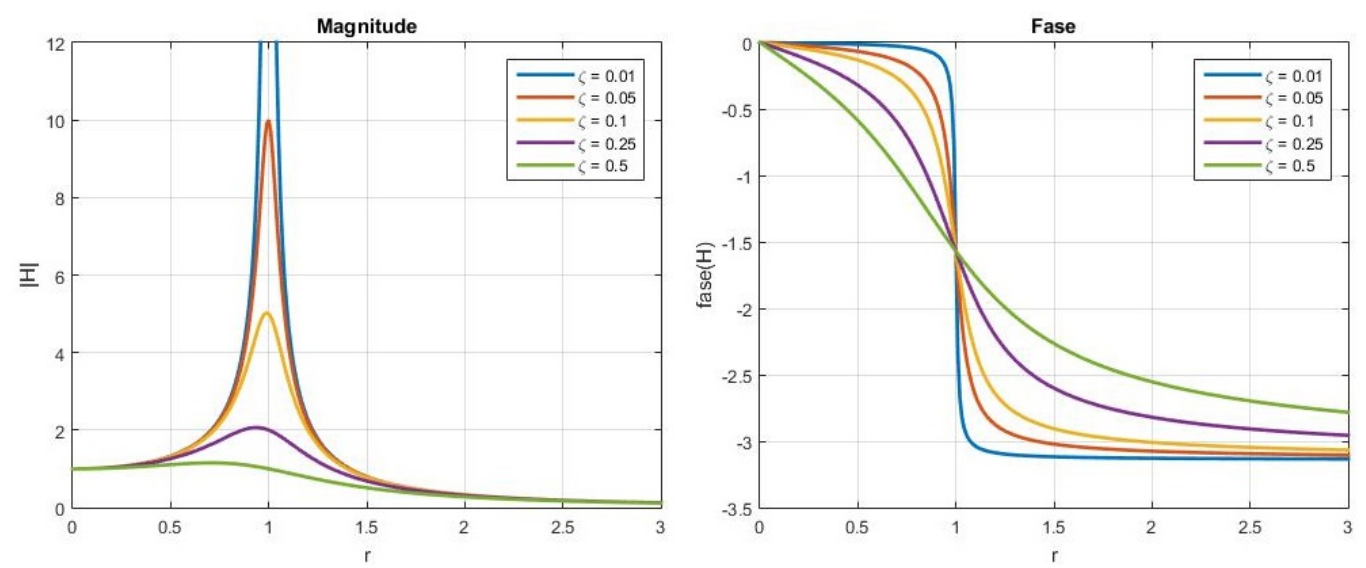

Figura 2.4: Gráfico Bode de um sistema SDoF

\section{Gráficos real × imaginário}

Ao separar a parte real da parte imaginária da FRF do deslocamento, obtêm-se

$$
\begin{aligned}
& \Re[\tilde{h}(\omega)]=\frac{k-\omega^{2} m}{\left(k-\omega^{2} m\right)^{2}+(\omega c)^{2}}=\frac{1-r^{2}}{\left(1-r^{2}\right)^{2}+(2 \zeta r)^{2}} \\
& \Im[\tilde{h}(\omega)]=\frac{-\omega c}{\left(k-\omega^{2} m\right)^{2}+(\omega c)^{2}}=\frac{-2 \zeta r}{\left(1-r^{2}\right)^{2}+(2 \zeta r)^{2}}
\end{aligned}
$$

Através da Eq. (2-28), percebe-se que, para a parte real da FRF ser zero, a frequência de forçamento $\omega$ precisa ser igual a $\omega_{n}$. Essa característica é então útil na identificação das frequências naturais. Os gráficos das partes reais e imaginárias da FRF são mostradas,novamente de forma adimensionais e com diferentes fatores de amortecimento, através da Figura 2.5.
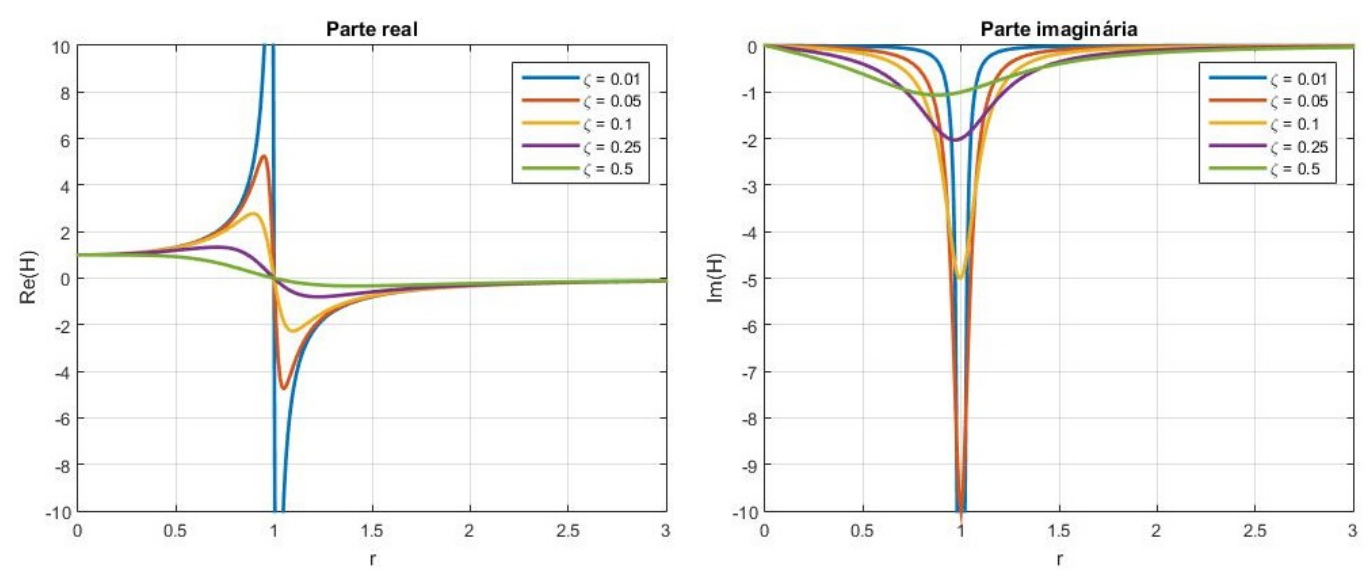

Figura 2.5: Representação gráfica da parte real e imaginária da FRF

\section{Gráfico Nyquist}

O gráfico de Nyquist consiste em plotar a parte imaginária da FRF em função da parte real. No caso de um sistema SDoF linear com amortecimento viscoso, 
percebe-se que os gráficos de Nyquist são aproximadamente um círculo. No entanto, pode-se mostrar que apenas a FRF da velocidade é de fato um. Ao explicitar suas partes real e imaginária, têm-se

$$
\begin{aligned}
& \Re\left[\tilde{h}_{v}(\omega)\right]=\frac{\omega^{2} c}{\left(k-m \omega^{2}\right)^{2}+(\omega c)^{2}} \\
& \Im\left[\tilde{h}_{v}(\omega)\right]=\frac{\omega\left(k-m \omega^{2}\right)}{\left(k-m \omega^{2}\right)^{2}+(\omega c)^{2}} .
\end{aligned}
$$

Através das Eqs. (2-30/2-31), pode-se verificar a relação

$$
\left(\Re\left[\tilde{h}_{v}(\omega)\right]-\frac{1}{2 c}\right)^{2}+\left(\Im\left[\tilde{h}_{v}(\omega)\right]\right)^{2}=\left(\frac{1}{2 c}\right)^{2} .
$$

A Eq. (2-32) representa um círculo centrado em $\left(\frac{1}{2 c}, 0\right)$ e com raio igual a $\frac{1}{2 c}$, como mostra a figura 2.6.

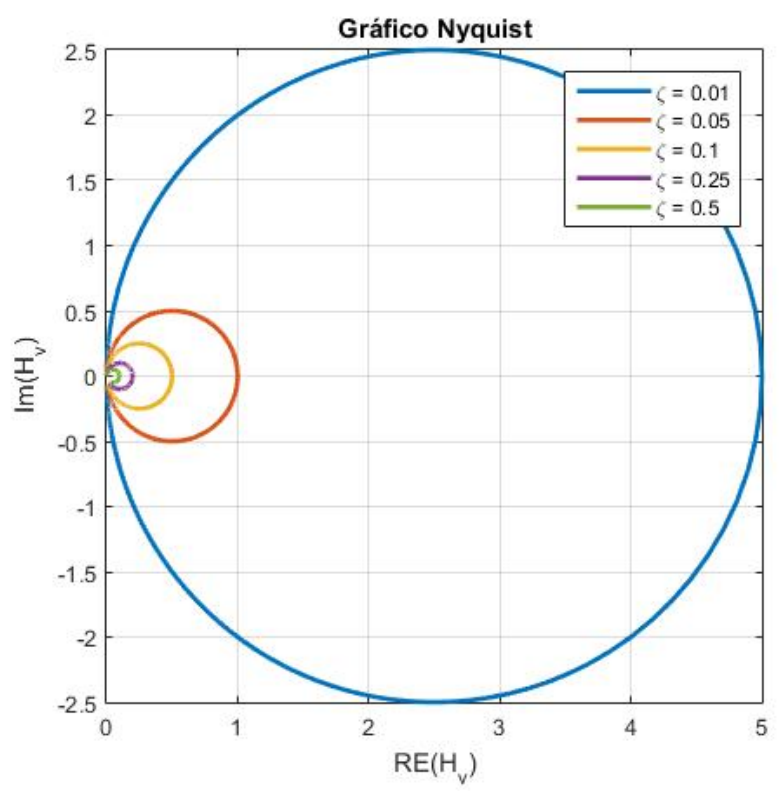

Figura 2.6: Gráfico de Nyquist

\subsection{3}

\section{Relação entre IRF e FRF}

Nas seções anteriores, mostrou-se que as funções de resposta ao impulso unitário e a de resposta em frequência caracterizam o sistema em função de seus parâmetros modais. Através da transformada de Fourier, é possível mostrar que ambas contém as mesmas informações sobre o sistema e estão apenas escritas em domínios diferentes. A seguir, mostra-se que, ao transformar a função de resposta ao impulso para o domínio da frequência, a função de resposta em frequência é obtida. Para isso, realiza-se primeiro a transformada de Laplace, 
e em seguida substitui-se a variável de Laplace $s$ por $i \omega$. A tabela 2.1 lista os principais pares da transformada de Laplace que serão utilizados.

Tabela 2.1: Propriedades da transformada de Laplace

\begin{tabular}{ccc}
\hline Descrição & $\begin{array}{c}\text { Domínio do tempo } \\
\boldsymbol{x}(\boldsymbol{t})\end{array}$ & $\begin{array}{c}\text { Domínio da Frequência } \\
\tilde{\boldsymbol{x}}(\boldsymbol{s})\end{array}$ \\
\hline Linearidade & $a x(t)+b f(t)$ & $a \tilde{x}(s)+b \tilde{f}(s)$ \\
Translação na frequência & $e^{i a t} x(t)$ & $\tilde{x}(s-a)$ \\
Transformada do seno & $x(t)=\sin (a t)$ & $\tilde{x}(s)=\frac{a}{s^{2}+a^{2}}$ \\
Convolução & $\int_{-\infty}^{+\infty} x(\tau) f(t-\tau) d \tau$ & $\tilde{x}(s) \tilde{f}(s)$ \\
\hline
\end{tabular}

Com as propriedade de linearidade, translação na frequência e da transformada do seno, a função de resposta ao impulso unitário definida em Eq. (2-13) é transformada para

$$
\begin{aligned}
\tilde{h}(s) & =\frac{1}{m \omega_{d}} \frac{\omega_{d}}{\left(s+\zeta \omega_{n}\right)^{2}+\omega_{d}^{2}} \\
& =\frac{1}{m\left[\left(s+\zeta \omega_{n}\right)^{2}+\omega_{d}^{2}\right]} .
\end{aligned}
$$

Substituindo a variável de Laplace $s$ por $i \omega$, obtêm-se a transformada de Fourier

$$
\begin{aligned}
\tilde{h}(\omega) & =\frac{1}{m\left[\left(i \omega+\zeta \omega_{n}\right)^{2}+\omega_{d}^{2}\right]} \\
& =\frac{1}{m\left[-\omega^{2}+2 \zeta \omega \omega_{n} i+\zeta^{2} \omega_{n}^{2}+\omega_{n}^{2}\left(1-\zeta^{2}\right)\right]} \\
& =\frac{1}{m\left[-\omega^{2}+2 \zeta \omega \omega_{n} i+\omega_{n}^{2}\right]} .
\end{aligned}
$$

Dividindo o numerado e denominador por $k$, a equação acima pode ser simplificada para

$$
\begin{aligned}
\tilde{h}(\omega) & =\frac{1 / k}{m / k\left[-\omega^{2}+2 \zeta \omega \omega_{n} i+\omega_{n}^{2}\right]} \\
& =\frac{1 / k}{1 / \omega_{n}^{2}\left[-\omega^{2}+2 \zeta \omega \omega_{n} i+\omega_{n}^{2}\right]} \\
& =\frac{1 / k}{-r^{2}+2 \zeta r i+1} .
\end{aligned}
$$

Ao comparar as Eqs. (2-20/2-35), percebe-se que ambas são iguais. Dessa forma, mostra-se a relação existente entre as funções IRF e FRF. 


\section{2}

\section{Análise Modal de um sistema MDoF}

Na grande maioria dos casos, uma modelagem utilizando apenas um grau de liberdade não resulta em uma boa descrição do movimento de um sistema. A modelagem utilizando apenas uma coordenada é em geral insuficiente e não é condiz com a realidade observada. Normalmente, quanto mais preciso os resultados precisam ser, mais coordenadas são necessárias no modelo do sistema. O motivo consiste no fato de que todo sistema é na verdade contínuo e por isso necessitaria infinitos graus de liberdade para obter as soluções exatas. Os sistemas que demandam mais de uma coordenada na sua modelagem são chamados de múltiplos graus de liberdade (MDoF) [25]. Esses modelos são mais complexos e sua análise modal é realizada como uma extensão da seção anterior.

Para os sistemas MDoF, um novo parâmetro chamado de modo de vibração surge para relacionar as diferentes coordenadas utilizadas no modelo. Os modos podem ser vetores reais ou complexos dependendo do tipo de amortecimento do sistema. Eles são responsáveis por caracterizar as formas espaciais nas quais o sistema vibra em uma dada frequência natural. Quando o amortecimento é negligenciado ou escrito de forma proporcional à massa e rigidez, os modos do sistema são definidos por coeficientes reais e são chamados de modos normais. Para um sistema com amortecimento generalizado, onde a modelagem proporcional não é mais possível, os modos do sistema passam a ser complexos. Isso resulta em uma defasagem entre as oscilações dos respectivos graus de liberdade, isto é, a amplitude máxima de vibração em diferentes posições da estrutura não ocorrem no mesmo tempo. Como visto a seguir, as respostas de um sistema podem ser sempre escritas como uma combinação linear dos modos. Isso significa que eles formam uma base para a resposta do sistema.

No início dessa seção serão apresentados o conceito de modos normais e sua importante propriedade de ortogonalidade. Em seguida, as FRFs e as FRIs são obtidas para um sistema com amortecimento proporcional. O principal objetivo de análise modal é conseguir caracterizar o sistema a partir dos parâmetros modais (frequências naturais, fatores de amortecimento e modos). Por isso mostra-se como essas FRFs e FRIs podem ser decompostas em função desses parâmetros.

Após apresentada a formulação para sistemas com amortecimento proporcional, será feita uma análise para sistemas com amortecimento viscoso generalizado. Para isso, uma modelagem chamada de estado de espaço precisa ser introduzida. No final desse capítulo, será mostrado que as FRFs e 
FRIs desse tipo de sistema também podem ser decompostas em função de seus parâmetros modais.

\subsection{1}

\section{Resposta livre de um sistema MDoF sem amortecimento}

A dinâmica de um sistema MDoS linear e sem amortecimento é dada por um sistema de equações diferenciais ordinárias escrita no forma matricial

$$
\mathcal{M} \ddot{\mathbf{q}}(t)+\mathcal{K} \mathbf{q}(t)=\mathbf{u}(t)
$$

onde $\mathcal{M}$ e $\mathcal{K} \in \mathbb{R}^{n \times n}$ são as matrizes de massa e rigidez, $\mathbf{q}(t)$ e $\mathbf{u}(t) \in \mathbb{R}^{n \times 1}$ os vetores de deslocamentos e forçamentos. A constante $n$ representa o número de graus de liberdade utilizado para modelar o sistema.

\section{Modos e frequência naturais}

Para a obtenção dos modos normais do sistema sem amortecimento e as respectivas frequências naturais, utiliza-se a hipótese de que a resposta livre é escrita como uma combinação linear dos modos. A resposta $\mathbf{q}(t)$ para a Eq. (2-36), com $\mathbf{u}(t)=0$, pode ser obtida pela expressão

$$
\mathbf{q}(t)=\boldsymbol{\psi} e^{i \omega t},
$$

onde $\boldsymbol{\psi}$ é um vetor de coeficiente reais. Ao substituir essa proposta de solução na Eq. (2-36), obtêm-se o seguinte problema de autovalor:

$$
\left(\mathcal{K}-\omega^{2} \mathcal{M}\right) \boldsymbol{\psi}=0
$$

A solução da Eq. (2-38) resulta em $n$ autovalores, $\omega_{r}^{2}$, e $n$ autovetores, $\boldsymbol{\psi}_{r}(r=$ $1,2, \ldots, n)$. Isso ocorre porque $\mathcal{K}$ e $\mathcal{M}$ são matrizes simétrica semi-positiva definida. As raízes quadrada dos autovalores, $\omega_{r}$, representam as frequência naturais do sistema e os autovetores $\boldsymbol{\psi}_{r}$ os modos. Supondo que as frequências naturais são distintas umas das outras, têm-se que os modos são ortogonais em relação a matriz de massa e rigidez. Assim, esses modos constroem uma base para o espaço resposta do sistema.

\section{Ortogonalidade dos modos}

A propriedade de ortogonalidade dos modos é dada em relação às matrizes de massa e rigidez. Ela pode ser demonstrada utilizando dois modos quaisquer e as respectivas frequências naturais na Eq. (2-37). O resultado utilizando o $i$-ésimo e o $j$-ésimo modo resultam em

$$
\left(\mathcal{K}-\omega_{i}^{2} \mathcal{M}\right) \boldsymbol{\psi}_{i}=0
$$




$$
\left(\mathcal{K}-\omega_{j}^{2} \mathcal{M}\right) \boldsymbol{\psi}_{j}=0 .
$$

Pré-multiplicando a Eq. (2-40) por $\boldsymbol{\psi}_{i}^{T}$ (onde ${ }^{T}$ significa transposta), obtêm-se

$$
\boldsymbol{\psi}_{i}^{T}\left(\mathcal{K}-\omega_{j}^{2} \mathcal{M}\right) \boldsymbol{\psi}_{j}=0
$$

Calculando a transposta ${ }^{1}$ da Eq. (2-39) e pós-multiplicando por $\boldsymbol{\psi}_{j}$, obtêm-se

$$
\boldsymbol{\psi}_{i}^{T}\left(\mathcal{K}-\omega_{i}^{2} \mathcal{M}\right) \boldsymbol{\psi}_{j}=0
$$

Ao subtrair a Eq. (2-41) da Eq. (2-42), obtêm-se

$$
\left(\omega_{j}^{2}-\omega_{i}^{2}\right) \boldsymbol{\psi}_{i}^{T} \mathcal{M} \boldsymbol{\psi}_{j}=0
$$

Supondo que as frequências naturais são distintas $\left(\omega_{i} \neq \omega_{j}\right)$, a Eq. (2-43) impõe que

$$
\boldsymbol{\psi}_{i}^{T} \mathcal{M} \boldsymbol{\psi}_{j}=0,
$$

Isso significa os modos são ortogonais em relação à matriz de massa. Substituindo a Eq. (2-44) na Eq. (2-41), obtêm-se

$$
\boldsymbol{\psi}_{i}^{T} \mathcal{K} \boldsymbol{\psi}_{j}=0
$$

que mostra a ortogonalidade dos modos também em relação à matriz de rigidez.

Criando-se uma matriz $\Omega^{2}$ cujos elementos da diagonal são as frequências naturais e uma matriz $\boldsymbol{\Psi}$ cujas colunas são os modos, tem-se

$$
\boldsymbol{\Omega}^{2}=\left[\begin{array}{cccc}
\omega_{1}^{2} & 0 & \ldots & 0 \\
0 & \omega_{2}^{2} & \ldots & 0 \\
\vdots & \vdots & \ddots & \vdots \\
0 & 0 & \ldots & \omega_{n}^{2}
\end{array}\right] \quad, \quad \Psi=\left[\begin{array}{llll}
\boldsymbol{\psi}_{1} & \boldsymbol{\psi}_{2} & \ldots & \boldsymbol{\psi}_{n}
\end{array}\right]
$$

Utilizando as matrizes acima, pode-se estender a Eq. (2-38) para

$$
\left(\mathcal{K}-\Omega^{2} \mathcal{M}\right) \Psi=0 .
$$

Pré-multiplicando a Eq. (2-47) por $\boldsymbol{\Psi}^{T}$, é possível diagonalizar as matrizes $\mathcal{M}$ e $\mathcal{K}$. Isto é,

$$
\begin{aligned}
\boldsymbol{\Psi}^{T}\left(\mathcal{K}-\Omega^{2} \mathcal{M}\right) \boldsymbol{\Psi} & =0 \\
\underbrace{\boldsymbol{\Psi}^{T} \mathcal{K} \boldsymbol{\Psi}}_{\mathbf{K}_{m}}-\boldsymbol{\Omega}^{2} \underbrace{\boldsymbol{\Psi}^{T} \mathcal{M} \boldsymbol{\Psi}}_{\mathbf{M}_{m}} & =0
\end{aligned}
$$

onde, $\mathbf{M}_{m}$ e $\mathbf{K}_{m}$ são matrizes diagonais chamadas de massa modal e rigidez modal, respectivamente. Seus elementos são definidos como

\footnotetext{
${ }^{1}$ Lembrando que $\mathcal{M}$ e $\mathcal{K}$ são simétricas e por isso $\mathcal{M}^{T}=\mathcal{M}$ e $\mathcal{K}^{T}=\mathcal{K}$. Através da
} álgebra linear, sabe-se que $(A B)^{T}=B^{T} A^{T}$. 


$$
\mathbf{M}_{m}=\left[\begin{array}{cccc}
m_{m, 1} & 0 & \ldots & 0 \\
0 & m_{m, 2} & \ldots & 0 \\
\vdots & \vdots & \ddots & \vdots \\
0 & 0 & \ldots & m_{m, n}
\end{array}\right], \quad \mathbf{K}_{m}=\left[\begin{array}{cccc}
k_{m, 1} & 0 & \ldots & 0 \\
0 & k_{m, 2} & \ldots & 0 \\
\vdots & \vdots & \ddots & \vdots \\
0 & 0 & \ldots & k_{m, n}
\end{array}\right]
$$

Através da Eq. (2-48) pode-se mostrar que as frequências naturais podem ser obtidas através de

$$
\Omega^{2}=\mathbf{K}_{m} \cdot \mathbf{M}_{m}^{-1}
$$

\section{Modos massa-normalizados}

O conjunto dos modos $\Psi$ calculado pelo do problema de autovalor (238) não é único, uma vez que seus múltiplos também são autovetores do problema. Uma representação única desses modos pode ser obtida através de uma normalização em função da matriz de massa modal. Isso é realizado através da equação

$$
\Theta=\Psi\left[\mathbf{M}_{m}\right]^{-\frac{1}{2}}
$$

Substituindo os modos normalizados $\Theta$ na Eq. (2-47) e pré multiplicando toda a equação por $\Theta^{T}$, têm-se como resultado

$$
\Theta^{T} \mathcal{K} \Theta-\Omega^{2} \underbrace{\Theta^{T} \mathcal{M} \Theta}_{\mathbf{I}}=0
$$

Isto é,

$$
\begin{gathered}
\boldsymbol{\Theta}^{T} \mathcal{M} \boldsymbol{\Theta}=\mathbf{I} \\
\boldsymbol{\Theta}^{T} \mathcal{K} \boldsymbol{\Theta}=\Omega^{2}
\end{gathered}
$$

Essas equações terão um papel importante na seção 2.2.3, onde será mostrado como as FRFs de um sistema MDoF podem ser decompostas em função dos parâmetros.

\section{Exemplo 2.1}

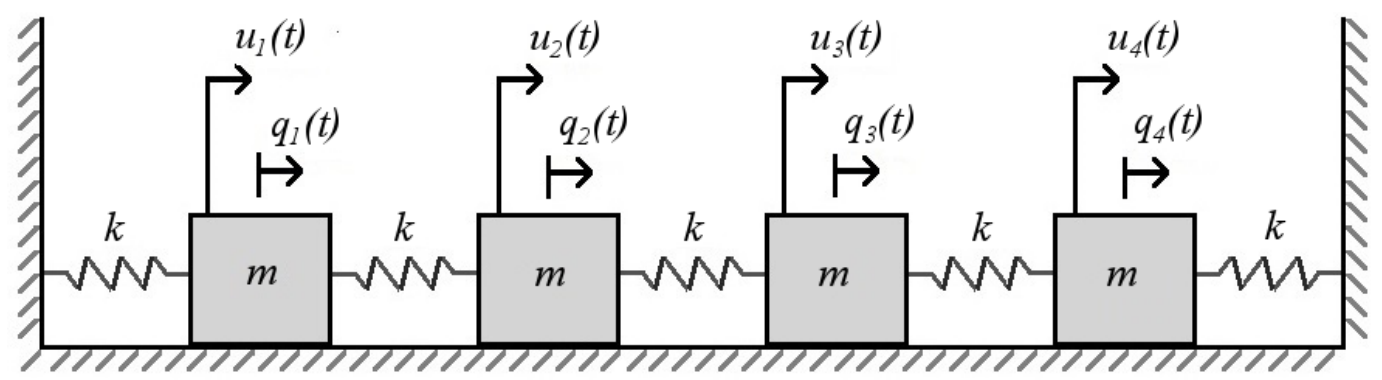

Figura 2.7: Exemplo de um sistem MDoF.

Para exemplificar as frequências naturais, modos e as matrizes de massa e rigidez modal, considere o sistema mostrado pela Figura 2.7. Adotando $k=1000 \mathrm{~N} / \mathrm{m}$ e $\mathrm{m} 1 \mathrm{~kg}$, obtêm-se as seguintes matrizes de massa e rigidez 


$$
\mathcal{M}=\left[\begin{array}{llll}
1 & 0 & 0 & 0 \\
0 & 1 & 0 & 0 \\
0 & 0 & 1 & 0 \\
0 & 0 & 0 & 1
\end{array}\right] \quad, \quad \mathcal{K}=\left[\begin{array}{cccc}
2000 & -1000 & 0 & 0 \\
-1000 & 2000 & -1000 & 0 \\
0 & -1000 & 2000 & -1000 \\
0 & 0 & -1000 & 2000
\end{array}\right]
$$

Substituindo essas matrizes na Eq. (2-36) e resolvendo o problema de autovalor da Eq. (2-38), obtêm-se

$$
\begin{aligned}
& \omega_{1}^{2}=381.967(\mathrm{rad} / \mathrm{s})^{2} \quad \omega_{2}^{2}=1381.97(\mathrm{rad} / \mathrm{s})^{2} \\
& \omega_{3}^{2}=2618.03(\mathrm{rad} / \mathrm{s})^{2} \quad \omega_{4}^{2}=3618.03(\mathrm{rad} / \mathrm{s})^{2}
\end{aligned}
$$

$e$

$$
\boldsymbol{\psi}_{1}=\left[\begin{array}{l}
0.37363 \\
0.60455 \\
0.60455 \\
0.37363
\end{array}\right] \quad \boldsymbol{\psi}_{2}=\left[\begin{array}{c}
-0.36180 \\
-0.22361 \\
0.22361 \\
0.36180
\end{array}\right] \quad \boldsymbol{\psi}=\left[\begin{array}{c}
-0.44721 \\
0.27639 \\
0.27639 \\
-0.44721
\end{array}\right] \quad \boldsymbol{\psi}=\left[\begin{array}{c}
-0.82706 \\
1.33821 \\
-1.33821 \\
0.82706
\end{array}\right]
$$

Escrevendo esses autovalores e autovetores na forma matricial, como definido em (2-46), têm-se

$$
\begin{gathered}
\boldsymbol{\Omega}^{2}=\left[\begin{array}{rccc}
381.967 & 0 & 0 & 0 \\
0 & 1381.97 & 0 & 0 \\
0 & 0 & 2618.03 & 0 \\
0 & 0 & 0 & 3618.03
\end{array}\right] \\
\Psi=\left[\begin{array}{cccc}
0.37363 & -0.36180 & -0.44721 & -0.82706 \\
0.60455 & -0.22361 & 0.27639 & 1.33821 \\
0.60455 & 0.22361 & 0.27639 & -1.33821 \\
0.37363 & 0.36180 & -0.44721 & 0.82706
\end{array}\right]
\end{gathered}
$$

Ao pré-multiplicar e pós-multiplicar $\mathcal{M}$ e $\mathcal{K}$ por $\boldsymbol{\Psi}^{T}$ e $\boldsymbol{\Psi}$, obtêm-se as matrizes modais $\mathbf{M}_{m}$ e $\mathbf{K}_{m}$.

$$
\begin{gathered}
\mathbf{M}_{m}=\boldsymbol{\Psi}^{T} \mathcal{M} \boldsymbol{\Psi}=\left[\begin{array}{cccc}
1.01017 & 0 & 0 & 0 \\
0 & 0.36180 & 0 & 0 \\
0 & 0 & 0.55279 & 0 \\
0 & 0 & 0 & 0.94966
\end{array}\right] \\
\mathbf{K}_{m}=\boldsymbol{\Psi}^{T} \mathcal{K} \boldsymbol{\Psi}=\left[\begin{array}{cccc}
385.851 & 0 & 0 & 0 \\
0 & 499.995 & 0 & 0 \\
0 & 0 & 1447.22 & 0 \\
0 & 0 & 0 & 3435.90
\end{array}\right]
\end{gathered}
$$

Os modos massa-normalizados podem ser obtidos através da Eq. (2-50), resultando em 


$$
\boldsymbol{\Theta}=\boldsymbol{\Psi}\left[\mathbf{M}_{m}\right]^{-\frac{1}{2}}=\left[\begin{array}{cccc}
0.37175 & -0.60150 & -0.60150 & -0.37175 \\
0.60150 & -0.37175 & 0.37175 & 0.60150 \\
0.60150 & 0.37175 & 0.37175 & -0.60150 \\
0.37175 & 0.60150 & -0.60150 & 0.37175
\end{array}\right]
$$

(Fim do exemplo)

\subsection{2}

\section{Resposta de um sistema MDoF com amortecimento proporcional}

A dinâmica de um sistema MDoF linear com amortecimento proporcional é ser expressa pela equação

$$
\mathcal{M} \ddot{\mathbf{q}}(t)+\mathcal{C}_{p} \dot{\mathbf{q}}(t)+\mathcal{K} \mathbf{q}(t)=\mathbf{u}(t)
$$

onde a matriz de amortecimento $\mathcal{C}_{p} \in \mathbb{R}^{n \times n}$ é definida como

$$
\mathcal{C}_{p}=\alpha \mathcal{M}+\beta \mathcal{K}
$$

Através dessa definição fica evidente que os modos do sistema conservativo associado também são ortogonais em relação a essa matriz de amortecimento, o que significa que $\mathcal{C}_{p}$ também pode ser diagonalizada através da equação

$$
\begin{aligned}
\boldsymbol{\Psi}^{T} \mathcal{C}_{p} \boldsymbol{\Psi} & =\alpha \boldsymbol{\Psi}^{T} \mathcal{M} \boldsymbol{\Psi}+\beta \boldsymbol{\Psi}^{T} \mathcal{K} \boldsymbol{\Psi} \\
& =\alpha \mathbf{M}_{m}+\beta \mathbf{K}_{m}=\mathbf{C}_{m}
\end{aligned}
$$

onde,

$$
\mathbf{C}_{m}=\left[\begin{array}{cccc}
c_{m, 1} & 0 & \ldots & 0 \\
0 & c_{m, 2} & \ldots & 0 \\
\vdots & \vdots & \ddots & \vdots \\
0 & 0 & \ldots & c_{m, n}
\end{array}\right]
$$

Utilizando os modos normais do sistema, uma mudança de variável pode ser criada, permitindo escrever as respostas do sistema em coordenadas modais. Essa mudança de variável é definida pela equação

$$
\mathbf{q}(t)=\mathbf{\Psi} \mathbf{z}(t) .
$$

Substituindo essa mudança de variável na Eq. (2-55), e pré-multiplicando por $\Psi^{T}$, obtêm-se como resultado

$$
\mathbf{M}_{m} \ddot{\mathbf{z}}(t)+\mathbf{C}_{m} \dot{\mathbf{z}}(t)+\mathbf{K}_{m} \mathbf{z}(t)=\mathbf{p}(t),
$$

onde $\mathbf{p}(t)=\boldsymbol{\Psi}^{T} \mathbf{u}(t)$. A Eq. (2-60) mostra que os modos normais podem ser usados para desacoplar o sistema. Como todas as matrizes na Eq. (2-60) são diagonais, o problema passa a ser composto por $n$ problemas de um grau de 
liberdade, como mostra a equação abaixo

$$
\left\{\begin{array}{c}
m_{m, 1} \ddot{z}_{1}(t)+c_{m, 1} \dot{z}_{1}(t)+k_{m, 1} z_{1}(t)=p_{1}(t) \\
m_{m, 2} \ddot{z}_{2}(t)+c_{m, 2} \dot{z}_{2}(t)+k_{m, 2} z_{2}(t)=p_{2}(t) \\
\vdots \\
m_{m, n} \ddot{z}_{n}(t)+c_{m, n} \dot{z}_{n}(t)+k_{m, n} z_{n}(t)=p_{n}(t)
\end{array} .\right.
$$

A solução destas equações, para condições iniciais nulas, foi estudada na seção 2.1 e tem a forma

$$
z_{r}(t)=h_{r}(t) * p_{r}(t),
$$

onde $h_{r}(t)$ é a resposta ao impulso unitário, definida pela Eq. (2-12), utilizando os os r-ésimos parâmetros modais $\left(m_{m, r}, c_{m, r}, k_{m, r} \rightarrow \omega_{r}, \zeta_{r}, \omega_{d, r}\right)$. Uma vez obtida todas as soluções $z_{r}(t)$, os resultados podem ser escritos nas coordenadas originais $\mathbf{q}(t)$ através da relação dada na Eq. (2-54).

\subsection{3}

\section{Decomposição modal das FRFs e IRFs de um sistema MDoF com amortecimento viscoso proporcional}

O objetivo desta seção é definir e mostrar como as funções de resposta em frequência (FRFs) e funções de resposta ao impulso (IRFs) podem ser escritas em função dos parâmetros modais. Essa propriedade é muito utilizada na análise modal experimental (EMA), onde as FRFs e/ou IRFs são obtidas experimentalmente e então os parâmetros modais são extraídos através de ajustes de curvas.

Utilizando a mudança de variável apresentada na Eq. (2-59), a resposta do sistema para um forçamento qualquer e condições iniciais nulas pode ser escrita como uma combinação linear dos modos, isto é

$$
\mathbf{q}(t)=\sum_{r=1}^{n} \boldsymbol{\psi}_{r} z_{r}(t)
$$

Substituindo as $n$ respostas do sistema em coordenadas modais, como definido na Eq. (2-62), a equação acima se transforma em

$$
\mathbf{q}(t)=\sum_{r=1}^{n} \boldsymbol{\psi}_{r} h_{r}(t) * p_{r}(t)
$$

Para manipular a Eq. (2-64) de forma mais simples, utiliza-se a transformada de Fourier, que transforma o operador de convolução em uma multiplicação. Assim, a Eq. (2-64) se transforma em

$$
\tilde{\mathbf{q}}(\omega)=\sum_{r=1}^{n} \boldsymbol{\psi}_{r} \tilde{h}_{r}(\omega) \tilde{p}_{r}(\omega)=\sum_{r=1}^{n} \boldsymbol{\psi}_{r} \tilde{h}_{r}(\omega) \boldsymbol{\psi}_{r}^{T} \tilde{\mathbf{u}}(\omega)
$$

As funções de resposta em frequência de cada modo, $\tilde{h}_{r}(\omega)$, quando escritas na 
forma fatorada (como definido pelas Eq. (2-20) e Eq. (2-21)), são equivalentes a

$$
\tilde{h}_{r}(\omega)=\frac{1}{2 i \omega_{d, r} m_{m, r}} \frac{1}{i \omega-\lambda_{r}}+\frac{1}{-2 i \omega_{d, r} m_{m, r}} \frac{1}{i \omega-\lambda_{r}^{*}},
$$

onde $\omega_{d, r}$ é a $r$-ésima frequência amortecida do sistema, $m_{m, r}$ é a $r$-ésima massa modal ( $r$-ésimo elemento na diagonal da matriz de massa modal $\mathbf{M}_{m}$ ) e $\lambda_{r}$ é o $r$-ésimo polo do sistema, definidos aqui novamente como

$$
\lambda_{r}=-\zeta_{r} \omega_{r}+i \omega_{d r}
$$

Substituindo a Eq. (2-66) na Eq. (2-65), obtêm-se

$$
\tilde{\mathbf{q}}(\omega)=\sum_{r=1}^{n}\left(\frac{\mathbf{A}_{r}}{i \omega-\lambda_{r}}+\frac{\mathbf{A}_{r}^{*}}{i \omega-\lambda_{r}^{*}}\right) \tilde{\mathbf{u}}(\omega),
$$

onde

$$
\mathbf{A}_{r}=\frac{\boldsymbol{\psi}_{r} \boldsymbol{\psi}_{r}^{T}}{2 i \omega_{d, r} m_{m, r}}=\frac{\boldsymbol{\psi}_{r} \boldsymbol{\psi}_{r}^{T}}{a_{r}}
$$

Através da Eq. (2-68), obtêm-se o termo que relaciona o sinal de forçamento com o sinal de resposta. Por estar tratando agora de um sistema de múltiplos graus de liberdade, essa relação é dada através de uma matriz, definida como

$$
\tilde{\mathbf{H}}(\omega)=\sum_{r=1}^{n}\left(\frac{\mathbf{A}_{r}}{i \omega-\lambda_{r}}+\frac{\mathbf{A}_{r}^{*}}{i \omega-\lambda_{r}^{*}}\right),
$$

de forma que

$$
\tilde{\mathbf{q}}(\omega)=\tilde{\mathbf{H}}(\omega) \tilde{\mathbf{u}}(\omega) \text {. }
$$

As componentes da matriz $\tilde{\mathbf{H}}(\omega)$, expressa na Eq. (2-70), são chamadas de funções de resposta em frequências (FRFs) de um sistema MDoF linear com amortecimento viscoso proporcional. Percebe-se que estas funções são definidas somente em pelos parâmetros modais do sistema (independente do forçamento). No formato em que as FRFs são apresentadas, uma importante característica dessas funções pode ser observada. Cada FRF (cada elemento da matriz $\tilde{\mathbf{H}}(\omega))$ é formada pela superposição da contribuição de cada modos. Quanto mais próximo a frequência $\omega$ estiver de uma das frequências naturais amortecidas, mais presente a parcela do respectivo modo estará na FRF.

Cada elemento da matriz $\tilde{\mathbf{H}}(\omega)$, definido a seguir por $\tilde{h}_{i j}(\omega)$, representa uma função que relaciona a amplitude de forçamento na posição $j$ com a amplitude da responta na posição $i$. Isto é, a matriz $\tilde{\mathbf{H}}(\omega)$ pode ser escrita na forma

$$
\tilde{\mathbf{H}}(\omega)=\left[\begin{array}{cccc}
\tilde{h}_{11}(\omega) & \tilde{h}_{12}(\omega) & \ldots & \tilde{h}_{1 n}(\omega) \\
\tilde{h}_{21}(\omega) & \tilde{h}_{22}(\omega) & \ldots & \tilde{h}_{2 n}(\omega) \\
\vdots & \vdots & \ddots & \vdots \\
\tilde{h}_{n 1}(\omega) & \tilde{h}_{n 2}(\omega) & \ldots & \tilde{h}_{n n}(\omega)
\end{array}\right]
$$

Se apenas uma força no ponto $j$ for aplicada e somente a amplitude da resposta no ponto $i$ for observada, a Eq. (2-71) se transforma em 


$$
\tilde{q}_{i}(\omega)=\tilde{h}_{i j}(\omega) \tilde{u}_{j}(\omega) \quad \Rightarrow \quad \tilde{h}_{i j}(\omega)=\frac{\tilde{q}_{i}(\omega)}{\tilde{u}_{j}(\omega)} .
$$

Neste caso, a relação entre as amplitudes têm a mesma definição da FRF para um sistema SDoF.

Como visto na seção 2.1, as funções de resposta ao impulso e as funções de resposta em frequência estão relacionadas através da transformada de Fourier. Como as FRFs já foram obtidas na Eq. (2-70), basta que a transformada inversa de Fourier seja aplicada para obter as IRFs. Realizando esta operação na Eq. (2-70), obtêm-se

$$
\mathbf{H}(t)=\sum_{r=1}^{n}\left(\mathbf{A}_{r} e^{\lambda_{r} t}+\mathbf{A}_{r}^{*} e^{\lambda_{r}^{*} t}\right) .
$$

A matriz acima contém então as as funções de resposta ao impulso unitário (IRFs). Essas funções relacionam os sinais de forçamento com os sinais de resposta (no domínio do tempo) através da equação

$$
\mathbf{q}(t)=\mathbf{H}(t) * \mathbf{u}(t)
$$

Através da Eq. (2-74), observar-se novamente que as funções de resposta ao impulso também podem ser escritas apenas em função dos parâmetros modais e que cada modo contribui com uma certa parcela na resposta final.

\section{Exemplo 2.2}

Considere o sistema do exemplo 2.1. Adiciona-se uma matriz de amortecimento proporcional conforme a Eq. (2-56), considerando $\alpha=0.2$ e $\beta=$ 0.0005. Utilizando o resultado da Eq. (2-57), a matriz de amortecimento pode ser diagonalizada pelos modos normais do sistema. Obtêm-se então

$$
\mathbf{C}_{m}=\left[\begin{array}{cccc}
0.3950 & 0 & 0 & 0 \\
0 & 0.2691 & 0 & 0 \\
0 & 0 & 0.5658 & 0 \\
0 & 0 & 0 & 1.1427
\end{array}\right]
$$

Como as matrizes $\mathbf{M}_{m}, \mathbf{C}_{m}$ e $\mathbf{K}_{m}$ são diagonais, a dinâmica do sistema passa a ser descrita por $n$ equações desacopladas, definidas pela Eq. (2-61). As frequências naturais e fatores de amortecimento podem ser calculadas através de

$$
\omega_{r}=\sqrt{\frac{k_{m, r}}{m_{m, r}}}, \quad \zeta_{r}=\frac{c_{m, r}}{2 \sqrt{k_{m, r} m_{m, r}}}, \quad r=1, \ldots, n .
$$

Os resultados para esse exemplo são mostrados na tabela 2.2, onde a frequência amortecida foi calculada através de $\omega_{d, r}=\omega_{r} \sqrt{1-\zeta_{r}^{2}}$.

Utilizando esses parâmetros modais, as FRFs do sistema podem ser calculadas pela Eq. (2-70). A Figura 2.8 mostra o gráfico da função $\tilde{h}_{11}(\omega)$ e a parcela de cada termo no somatório da Eq. (2-70), isto é, a parcela de 
Tabela 2.2: Parâmetros modais do sistema com amortecimento proporcional

\begin{tabular}{ccccc}
\hline & $1^{\mathrm{O}}$ modo & $2^{\mathrm{O}}$ modo & $3^{\mathrm{O}}$ modo & $4^{\mathrm{O}}$ modo \\
\hline$\omega_{r}(\mathrm{rad})$ & 19.5440 & 37.1748 & 51.1667 & 60.1501 \\
$\zeta_{r}(\%)$ & 1.00 & 1.20 & 1.47 & 1.67 \\
$\omega_{d, r}(\mathrm{rad})$ & 19.5430 & 37.1721 & 51.1612 & 60.1417 \\
\hline
\end{tabular}

contribuição de cada modo. Como previsto, na proximidade de cada frequência natural, existe um modo que se torna dominante. Sua magnitude aumenta bastante em relação às outras curvas nesta região, caracterizando o fenômeno de ressonância. (Fim do exemplo)

Decomposição modal da função $\mathrm{h}_{11}$

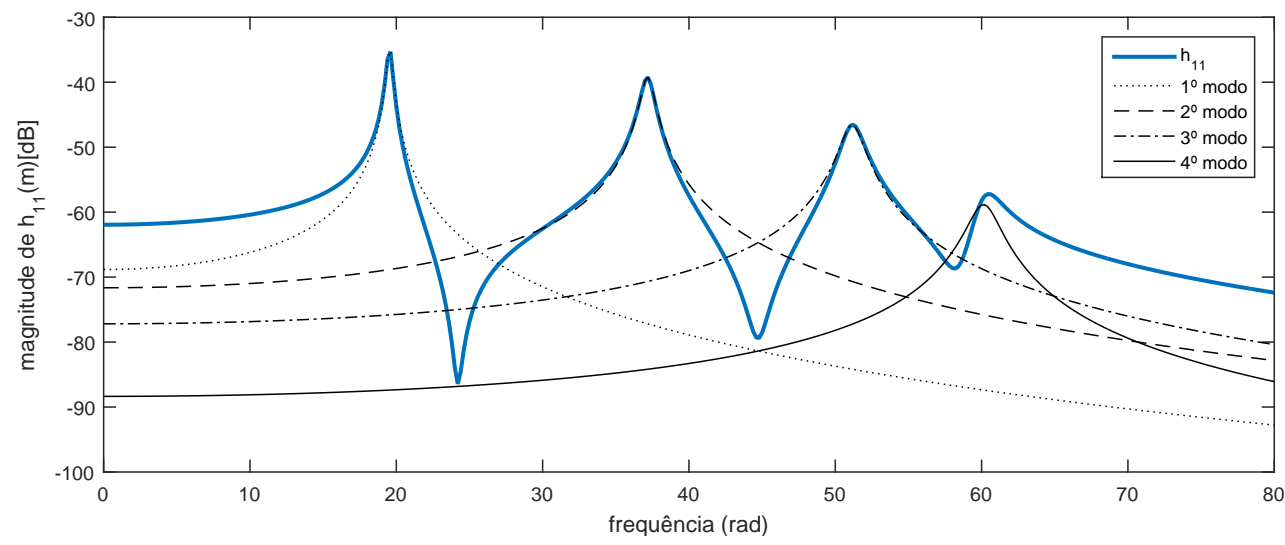

Figura 2.8: Decomposição modal da FRF de um sistema com amortecimento viscoso proporcional.

\subsection{4}

Sistemas MDoF com amortecimento viscoso generalizado

Considere um sistema MDoF linear cuja dinâmica é dada por:

$$
\mathcal{M} \ddot{\mathbf{q}}(t)+\mathcal{C} \dot{\mathbf{q}}(t)+\mathcal{K} \mathbf{q}(t)=\mathbf{u}(t) .
$$

Quando a matriz de amortecimento não pode ser modelada de forma proporcional à matriz de massa e rigidez, a dinâmica do sistema não pode ser desacoplada utilizando os modos normais. Isso acontece porque, o produto $\Psi^{T} \mathcal{C} \Psi$ não resulta em uma matriz diagonal. A alternativa para obter-se a resposta da Eq. (2-76) e decompor as FRFs e IFR em relação aos parâmetros modais consiste em transformar a Eq. (2-76) em um formato de espaço de estado descrito a seguir $[2,26,27]$.

Ao criar a nova variável de estado $\mathbf{x}(t)=\left[\mathbf{q}(t)^{T} \dot{\mathbf{q}}(t)^{T}\right]^{T}$, a Eq. $(2-76)$ 
pode ser reescrita na forma

$$
\begin{aligned}
\mathbf{A} \dot{\mathbf{x}}(t)+\mathbf{B} \mathbf{x}(t) & =\mathbf{f}(t) \\
\mathbf{q}(t) & =\mathbf{C x}(t)
\end{aligned}
$$

onde,

$$
\mathbf{A}=\left[\begin{array}{cc}
\mathcal{C} & \mathcal{M} \\
\mathcal{M} & 0
\end{array}\right], \mathbf{B}=\left[\begin{array}{cc}
\mathcal{K} & 0 \\
0 & -\mathcal{M}
\end{array}\right], \mathbf{f}(t)=\left[\begin{array}{c}
\mathbf{u}(t) \\
0
\end{array}\right], \mathbf{C}=\left[\begin{array}{ll}
\mathbf{I} & 0
\end{array}\right] .
$$

A matriz $\mathbf{I} \in \mathbb{R}^{\mathbf{n} \times \mathbf{n}}$ consiste na matriz identidade. Como pode ser observado, a Eq. (2-77) consiste em uma equação diferencial ordinária de primeira ordem, e não mais de segunda como na Eq. (2-76). No entanto, desvantagem está na dimensão das matrizes que compõem o modelo de espaço de estado, que passa a ser $2 n \times 2 n$.

A solução livre para o modelo de espaço de estado pode ser obtida considerando uma solução na forma

$$
\mathbf{x}(t)=\phi e^{\lambda t}
$$

Substituindo essa solução na Eq. (2-77), o seguinte problema de autovalor é criado:

$$
\lambda \mathbf{A} \phi+\mathbf{B} \phi=0
$$

Como os autovetores deste problema têm dimensão $2 n$, ele contém informações sobre o modos de vibração do deslocamento e velocidade, simultaneamente. Isso significa que esses autovetores são escritos na forma

$$
\phi_{r}=\left[\begin{array}{c}
\boldsymbol{\psi}_{r} \\
\lambda \boldsymbol{\psi}_{r}
\end{array}\right], \quad r=1, \ldots, 2 n
$$

As $n$ primeiras linhas representam o modo do deslocamento enquanto as últimas $n$ representam o modo de velocidade. Se considerarmos o caso onde os amortecimentos modais são subcríticos $\left(\zeta_{r}<1\right)$, pode-se mostrar que o problema de autovalor definido na Eq. (2-80) irá resultar $2 n$ autovalores e autovetores complexos, escritos na forma de pares conjugados.

Ao substituir a Eq. (2-81) na Eq. (2-79), e o respectivo resultado na Eq. (2-77), com $\mathbf{f}(t)=0$, observa-se a formação da seguinte equação

$$
\left(\lambda_{r}^{2} \mathcal{M}+\lambda_{r} \mathcal{C}+\mathcal{K}\right) \boldsymbol{\psi}_{r}=0
$$

Uma importante característica dessa equação é o fato dos modos serem compostos de termos complexos e de que se a Eq. (2-82) for multiplicada por $\boldsymbol{\psi}_{r}^{T}$, as matrizes $\mathcal{M}, \mathcal{C}$ e $\mathcal{K}$ não serão diagonalizadas.

No entanto, pode-se mostrar que os autovetores $\phi_{r}$ são ortogonais em 
relação às matrizes A e B. Segundo o mesmo desenvolvimento apresentado na seção 2.2.1, essa ortogonalidade pode ser mostrada utilizando os autovetores $\phi_{i}$ e $\phi_{j}$. O resultado são as duas equações

$$
\begin{gathered}
\left(\lambda_{i} \mathbf{A}+\mathbf{B}\right) \phi_{i}=0 \\
\left(\lambda_{j} \mathbf{A}+\mathbf{B}\right) \phi_{j}=0 .
\end{gathered}
$$

Ao pré-multiplicar a Eq. (2-84) por $\phi_{i}^{T}$, obtêm-se

$$
\phi_{i}^{T}\left(\lambda_{j} \mathbf{A}+\mathbf{B}\right) \phi_{j}=0 .
$$

Calculando a transposta ${ }^{1}$ da Eq. (2-83) e pós-multiplicando por $\phi_{j}$, obtêm-se

$$
\phi_{i}^{T}\left(\lambda_{i} \mathbf{A}+\mathbf{B}\right) \phi_{j}=0 .
$$

Subtraindo a Eq. (2-85) da Eq. (2-86), têm-se como resultado a equação

$$
\left(\lambda_{i}-\lambda_{j}\right) \boldsymbol{\phi}_{i}^{T} \mathbf{A} \boldsymbol{\phi}_{j}=0 .
$$

Supondo que $\lambda_{i} \neq \lambda_{j}$, conclui-se que $\boldsymbol{\phi}_{i}^{T} \mathbf{A} \phi_{j}=0$. Utilizando esse resultado na Eq. (2-85), conclui-se também que $\boldsymbol{\phi}_{i}^{T} \mathbf{B} \phi_{j}=0$.

Através dessa propriedade de ortogonalidade, os coeficientes modais do $r$-ésimo modo associados às matrizes $\mathbf{A}$ e $\mathbf{B}$ podem ser definidos como

$$
\left\{\begin{array}{l}
a_{r}=\boldsymbol{\phi}_{r}^{T} \mathbf{A} \boldsymbol{\phi}_{r}=2 \lambda_{r} \boldsymbol{\psi}_{r}^{T} \mathcal{M} \boldsymbol{\psi}_{r}+\boldsymbol{\psi}_{r}^{T} \mathcal{C} \boldsymbol{\psi}_{r} \\
b_{r}=\boldsymbol{\phi}_{r}^{T} \mathbf{B} \boldsymbol{\phi}_{r}=\boldsymbol{\psi}_{r}^{T} \mathcal{K} \boldsymbol{\psi}_{r}-\lambda_{r}^{2} \boldsymbol{\psi}_{r}^{T} \mathcal{M} \boldsymbol{\psi}_{r}
\end{array} \quad, \quad r=1, \ldots, 2 n\right.
$$

Ao substituir o $r$-ésimo modo na Eq. (2-80), e pré-multiplicar toda a equação pelo mesmo modo transposto, o resultado encontrado é

$$
\lambda_{r} \boldsymbol{\phi}_{r}^{T} \mathbf{A} \phi_{r}+\boldsymbol{\phi}_{r}^{T} \mathbf{B} \phi_{r}=\lambda_{r} a_{r}+b_{r}=0 .
$$

Ao substituir na equação acima as definições dos coeficientes modais $a_{r}$ e $b_{r}$ apresentados na Eq. (2-88), percebe-se que a Eq. (2-89) é na verdade a equação característica do $r$-ésimo modo, isto é

$$
\begin{gathered}
\lambda_{r} a_{r}+b_{r}=0 \\
\mathbb{1} \\
\lambda_{r}^{2} \boldsymbol{\psi}_{r}^{T} \mathcal{M} \boldsymbol{\psi}_{r}+\lambda_{r} \boldsymbol{\psi}_{r}^{T} \mathcal{C} \boldsymbol{\psi}_{r}+\boldsymbol{\psi}_{r}^{T} \mathcal{K} \boldsymbol{\psi}_{r}=0 .
\end{gathered}
$$

Utilizando todos os coeficientes modais $a_{r}$ e $b_{r}$ na Eq. (2-90), os resultados são os mesmos $2 n$ autovalores (polos do sistema) obtidos pelo problema de autovalor da Eq. (2-80). Esses autovalores também aparecem em pares

\footnotetext{
${ }^{1}$ Nota-se que como $\mathcal{M}, \mathcal{C}$ e $\mathcal{K}$ são simétricas, $\mathbf{A}^{T}=\mathbf{A}$ e $\mathbf{B}^{T}=\mathbf{B}$
} 
complexos conjugados, definidos a seguir como

$$
\left\{\begin{array}{l}
\lambda_{r} \\
\lambda_{r}^{*}
\end{array}=\sigma_{r} \pm i \omega_{d r}\right.
$$

Ao fazer uma analogia com os polos definidos pela Eq. (2-67) para o sistema com amortecimento viscoso proporcional, percebe-se que parâmetros modais equivalentes podem ser calculados através desses autovalores:

$$
\begin{gathered}
\omega_{n r}=\sqrt{\lambda_{r} \lambda_{r}^{*}}=\sqrt{\sigma_{r}^{2}+\omega_{d, r}^{2}} \\
\zeta_{r}=-\frac{\sigma_{r}}{\omega_{r}} .
\end{gathered}
$$

A Eq. (2-91) pode então ser reescrita como

$$
\left\{\begin{array}{l}
\lambda_{r} \\
\lambda_{r}^{*}
\end{array}=-\zeta_{r} \omega_{r} \pm i \omega_{d, r} .\right.
$$

Uma importante observação deve ser feita em relação a frequência natural equivalente definida na Eq. (2-92). Percebe-se que a ela é função do amortecimento do sistema, o que não é coerente com a definição previamente dada para a frequência natural.

Assim, como feito para a resposta do sistema com amortecimento proporcional, os estados $\mathbf{x}(t)$ do sistema também podem ser escritos como uma combinação linear dos autovetores $\phi_{r}$. Isso é feito através da mudança de variável

$$
\mathbf{x}(t)=\mathbf{\Phi} \mathbf{w}(t)=\sum_{r=1}^{2 n} \boldsymbol{\phi}_{r} w_{r}(t),
$$

onde $\boldsymbol{\Phi}=\left[\phi_{1} \phi_{2} \ldots \phi_{2 n}\right]$ é a base modal e $\mathbf{w}(t)$ é o vetor dos estados em coordenadas modais. Substituindo essa mudança de variável na Eq. (2-77), e multiplicando o resultado por $\boldsymbol{\Phi}^{T}$, as equações de espaço de estado são desacopladas e resultam em um conjunto de $2 n$ equações diferenciais ordinárias de primeira ordem

$$
\left\{\begin{array}{c}
a_{1} \dot{w}_{1}(t)+b_{1} w_{1}(t)=\phi_{1}^{T} \mathbf{f}(t) \\
a_{2} \dot{w}_{2}(t)+b_{2} w_{2}(t)=\phi_{2}^{T} \mathbf{f}(t) \\
\vdots \\
a_{2 n} \dot{w}_{2 n}(t)+b_{2 n} w_{2 n}(t)=\phi_{2 n}^{T} \mathbf{f}(t)
\end{array}\right.
$$

Cada uma dessas equações pode ser transformada para o domínio da frequência através da transformada de Fourier, resultando em

$$
\left(i \omega a_{r}+b_{r}\right) \tilde{w}_{r}(\omega)=\phi_{r}^{T} \tilde{\mathbf{f}}(\omega), \quad r=1, \ldots, 2 n .
$$


Explicitando o termo $\tilde{w}_{r}(\omega)$ e utilizando a expressão $\lambda_{r}=-b_{r} / a_{r}$ calculada a partir da Eq. (2-90), a Eq. (2-97) se transforma em

$$
\tilde{w}_{r}(\omega)=\frac{1}{a_{r}\left(i \omega-\lambda_{r}\right)} \boldsymbol{\phi}_{r}^{T} \tilde{\mathbf{f}}(\omega)
$$

Realizando agora a transformada de Fourier no dois lados da Eq. (2-95), e substituindo as funções $\tilde{w}_{r}(\omega)$ obtidos pela Eq. (2-98), a seguinte relação entre os sinais de força e estado é obtida

$$
\tilde{\mathbf{x}}(\omega)=\sum_{r=1}^{2 n} \phi_{r} \tilde{w}_{r}(\omega)=\sum_{r=1}^{2 n} \frac{\phi_{r} \phi_{r}^{T}}{a_{r}\left(i \omega-\lambda_{r}\right)} \tilde{\mathbf{f}}(\omega) .
$$

Como tanto os autovalores (polos) $\lambda_{r}$ quanto os autovetores (modos) $\boldsymbol{\psi}_{r}$ formam $n$ pares de complexos conjugados, a Eq. (2-99) pode ser reescrita como

$$
\tilde{\mathbf{x}}(\omega)=\sum_{r=1}^{n}\left(\frac{\phi_{r} \phi_{r}^{T}}{a_{r}\left(i \omega-\lambda_{r}\right)}+\frac{\phi_{r}^{*} \phi_{r}^{* T}}{a_{r}^{*}\left(i \omega-\lambda_{r}^{*}\right)}\right) \tilde{\mathbf{f}}(\omega)=\tilde{\mathbf{H}}_{x}(\omega) \tilde{\mathbf{f}}(\omega) .
$$

A Eq. (2-100) define a matriz $\tilde{\mathbf{H}}_{x}(\omega)$ com a função de resposta em frequência do modelo de espaço de estado do sistema, já que ela relaciona os sinais de forçamento com os estados do sistema. Como o objetivo é obter a função de resposta em frequência apenas do deslocamento do sistema e não do estado, basta que $n$ primeiras linhas e colunas da matriz $\tilde{\mathbf{H}}_{x}(\omega)$ sejam extraídas. Isso pode ser visto reescrevendo a Eq. (2-100) como

$$
\underbrace{\left[\begin{array}{c}
\tilde{\mathbf{q}}(\omega) \\
\tilde{\mathbf{q}}(\omega)
\end{array}\right]}_{\tilde{\mathbf{x}}(\omega)}=\underbrace{\left[\begin{array}{cc}
\tilde{\mathbf{H}}_{11}(\omega) & \tilde{\mathbf{H}}_{12}(\omega) \\
\tilde{\mathbf{H}}_{21}(\omega) & \tilde{\mathbf{H}}_{22}(\omega)
\end{array}\right]}_{\tilde{\mathbf{H}}_{x}(\omega)} \underbrace{\left[\begin{array}{c}
\tilde{\mathbf{u}}(\omega) \\
0
\end{array}\right]}_{\tilde{\mathbf{f}}(\omega)} .
$$

A função de resposta em frequência de um sistema linear com múltiplos graus de liberdade e amortecimento viscoso generalizado é dado então pela equação

$$
\tilde{\mathbf{H}}(\omega)=\tilde{\mathbf{H}}_{11}(\omega)=\sum_{r=1}^{n}\left(\frac{\mathbf{A}_{r}}{i \omega-\lambda_{r}}+\frac{\mathbf{A}_{r}^{*}}{i \omega-\lambda_{r}^{*}}\right),
$$

onde agora,

$$
\mathbf{A}_{r}=\frac{\boldsymbol{\psi}_{r} \boldsymbol{\psi}_{r}^{T}}{a_{r}}
$$

Vale lembrar que os modos $\boldsymbol{\psi}_{r}$ são obtidos pela Eq. (2-81) com as $n$ primeiras linhas do autovetor $\phi_{r}$. Nas FRFs da Eq. (2-102), todos os modos, coeficientes modais $a_{r}$ e polos são grandezas complexas. O mesmo não ocorre quando o sistema é modelado com amortecimento proporcional, onde apenas os polos são complexos. Supõe-se nesta dissertação que os modos possuem baixa complexidade, o que permite que os mesmos sejam aproximados e representados graficamente pelas partes reais.

Para obter as funções de resposta ao impulso unitário, basta que a transformada inversa de Fourier seja aplicada na Eq.(2-102), resultando em 


$$
\mathbf{H}(t)=\sum_{r=1}^{n}\left(\mathbf{A}_{r} e^{\lambda_{r} t}+\mathbf{A}_{r}^{*} e^{\lambda_{r}^{*} t}\right)
$$

onde neste caso $\mathbf{A}_{r}$ é definida como na Eq.(2-103).

Para um sistema com amortecimento viscoso generalizado, foi novamente possível decompor as FRFs e IRFs em função dos parâmetros modais do sistema, Eqs. (2-102/2-104) respectivamente. Além disso, esta formulação deixa claro que as FRFs e IRFs também são escritas como a superposição das contribuições de cada modo.

\section{Exemplo 2.3}

Considere novamente o sistema do Exemplo 2.2. No entanto, alguns coeficientes da matriz de amortecimento proporcional são alterados para que a mesma perca a proporcionalidade. Faz-se $\mathcal{C}_{34}=\mathcal{C}_{43}=0$, ou seja, a matriz de amortecimento passa a ser

$$
\mathcal{C}=\left[\begin{array}{cccc}
1.2 & -0.5 & 0 & 0 \\
-0.5 & 1.2 & -0.5 & 0 \\
0 & -0.5 & 1.2 & 0 \\
0 & 0 & 0 & 1.2
\end{array}\right]
$$

Como matriz não é proporcional à matriz de massa e rigidez, não pode ser diagonalizada pelos modos normais do sistema. Isto pode ser verificado pela expressão

$$
\boldsymbol{\Psi}^{T} \mathcal{C} \boldsymbol{\Psi}=\left[\begin{array}{cccc}
0.6146 & 0.2500 & -0.1118 & 0 \\
0.2500 & 1.1146 & 0 & -0.1118 \\
-0.1118 & 0 & 1.2854 & 0.2500 \\
0 & -0.1118 & 0.2500 & 1.7854
\end{array}\right]
$$

onde $\boldsymbol{\Psi}$ foi calculada através da Eq. (2-46). A alternativa para achar a solução da dinâmica do sistema é calcular as matrizes de espaço de estado $\mathbf{A} e \mathbf{B} e$ resolver o problema de autovalor definido pela Eq. (2-80). Os 8 autovalores $e$ autovetores calculados para este exemplo, escrito em pares de complexos 
conjugados, são

$$
\begin{array}{r}
\lambda_{1,2}=0.8927 \pm 60.1414 i, \quad \lambda_{3,4}=0.6427 \pm 51.1641 i \\
\lambda_{5,6}=0.5573 \pm 37.1696 i, \quad \lambda_{7,8}=0.3073 \pm 19.5422 i \\
\phi_{1,2}=\left[\begin{array}{c}
-0.0001 \pm 0.0101 i \\
0.0003 \pm 0.0163 i \\
-0.0006 \pm 0.0163 i \\
0.0006 \pm 0.0101 i \\
0.6061 \pm 0.0038 i \\
-0.9804 \pm 0.0019 i \\
0.9798 \pm 0.0202 i \\
-0.6053 \pm 0.0256 i
\end{array}\right], \phi_{3,4}=\left[\begin{array}{c}
0.0007 \pm 0.0191 i \\
-0.0007 \pm 0.0118 i \\
-0.0002 \pm 0.0118 \\
0.0004 \pm 0.0191 i \\
-0.9758 \pm 0.0242 i \\
0.6028 \pm 0.0261 i \\
0.6035 \pm 0.0012 i \\
-0.9763 \pm 0.0088 i
\end{array}\right] \\
\phi_{5,6}=\left[\begin{array}{c}
-0.0010 \pm 0.0263 i \\
-0.0008 \pm 0.0162 i \\
0.0003 \pm 0.0163 i \\
0.0007 \pm 0.0263 i \\
0.9774 \pm 0.0226 i \\
0.6039 \pm 0.0221 i \\
-0.6043 \pm 0.0039 i \\
-0.9777 \pm 0.0114 i
\end{array}\right], \phi_{7,8}=\left[\begin{array}{c}
0.0016 \pm 0.0302 i \\
-0.0028 \pm 0.0488 i \\
-0.0031 \pm 0.0488 i \\
-0.0021 \pm 0.0302 i \\
0.5902 \pm 0.0229 i \\
0.9549 \pm 0.0396 i \\
0.9547 \pm 0.0453 i \\
0.5899 \pm 0.0322 i
\end{array}\right]
\end{array}
$$

Ao realizar o produto $\boldsymbol{\Phi}^{T} \mathbf{A} \boldsymbol{\Phi}$, os coeficiente modais $a_{r}$ podem ser calculados conforme definido pela Eq. (2-88). Os resultados são

$$
\begin{aligned}
& a_{1,2}=-0.0049 \pm 0.0881 i, a_{3,4}=-0.0064 \pm 0.1027 i \\
& a_{5,6}=-0.0095 \pm 0.1418 i, a_{7,8}=-0.0313 \pm 0.2564 i .
\end{aligned}
$$

A partir dos polos $\lambda_{r}$ é possível então calcular os parâmetros modais do sistema pelas Eqs. (2-92/2-93). Os resultados são mostrados na tabela 2.3. Percebe-se que eles são muitos próximos aos já obtidos no Exemplo 2.2, mesmo que metodologia utilizada tenha sido totalmente diferente.

Tabela 2.3: Parâmetros modais do sistema com amortecimento viscoso generalizado

\begin{tabular}{ccccc}
\hline & $1^{\mathrm{O}}$ modo & $2^{\mathrm{O}}$ modo & $3^{\mathrm{O}}$ modo & $4^{\mathrm{O}}$ modo \\
\hline$\omega_{n r}(\mathrm{rad})$ & 19.5446 & 37.1737 & 51.1682 & 60.1480 \\
$\zeta_{r}(\%)$ & 1.57 & 1.50 & 1.26 & 1.48 \\
$\omega_{d r}(\mathrm{rad})$ & 19.5422 & 37.1696 & 51.1641 & 60.1414 \\
\hline
\end{tabular}

As funções de resposta em frequência podem ser calculadas a partir da Eq. (2-102). A figura 2.9 mostra o gráfico da função $h_{11}(\omega)$ e a parcela de 
cada termo do somatório da Eq. (2-102), isto é, a parcela de contribuição de cada modo. Assim no caso de amortecimento proporcional, próximo a cada frequência natural existe um modo que se torna dominante. Isto é, sua magnitude aumenta consideravelmente, caracterizando o fenômeno de ressonância. (Fim do exemplo)

Decomposição modal da função $h_{11}$

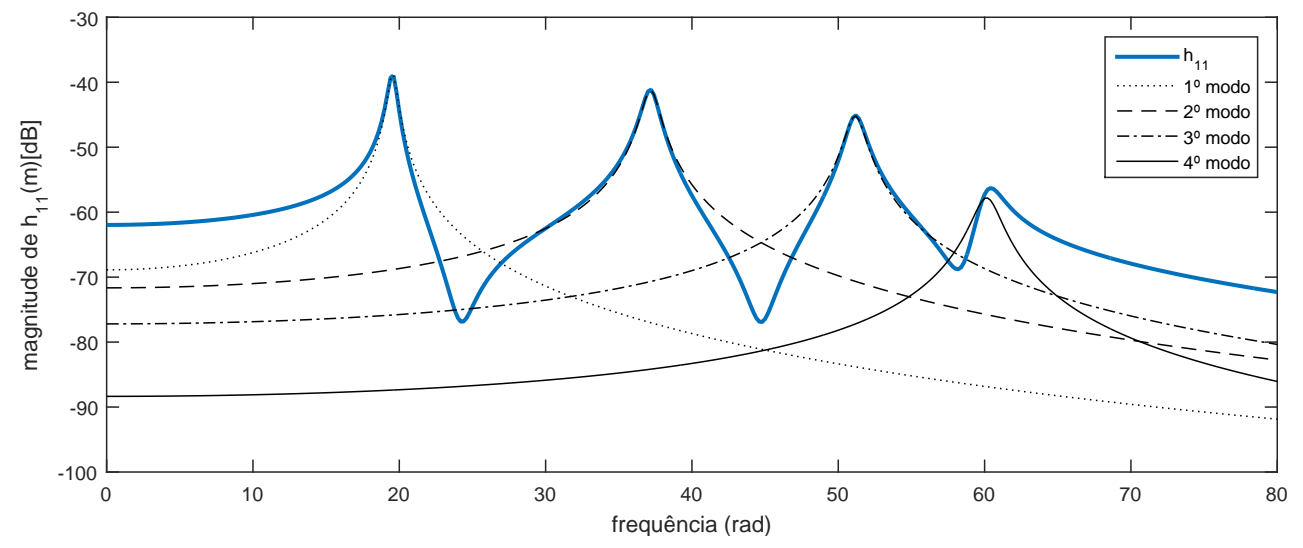

Figura 2.9: Decomposição modal da FRF de um sistema com amortecimento viscoso generalizado. 


\section{3}

\section{Sinais aleatórios}

O principal objetivo deste capítulo é mostrar como a análise modal pode ser aplicada em sistemas submetidos a forçamentos aleatórios (condições operacionais). Quando um sistema não se encontra em um laboratório, diversas forças do ambiente podem excitá-lo. Exemplos desses tipos de forçamento são o vento, ondas, tráfico de automóveis, vibrações de equipamentos ao redor, etc. Quando contribuição dessas forças são relevantes na resposta do sistema, elas não podem ser negligenciadas. Por isso, elas necessitam ser contabilizadas na dinâmica do sistema. Muitas vezes essas forças do ambiente são as únicas formas possíveis de excitação, como por exemplo em grandes estruturas civis.

Os sinais de entrada (forçamento) e saída (resposta) são modelados neste caso por processos estocásticos. Na primeira parte desse capítulo serão apresentadas as definições de duas estatísticas desses processos, as funções de correlação (domínio do tempo) e densidade espectral de potência (domínio da frequência) [28].

Em seguida, busca-se estabelecer a relação existente entre as estatísticas dos sinais de entrada e as estatísticas dos sinais de saída [30, 29], como mostra a Figura 3.1. No caso determinístico do capítulo anterior, essa relação era dada pelas funções de resposta ao impulso e função de resposta em frequência. Para o caso estocástico, esta relação é dada por funções que representam a energia do sistema, seja ela escrita no domínio do tempo quanto no domínio da frequência.

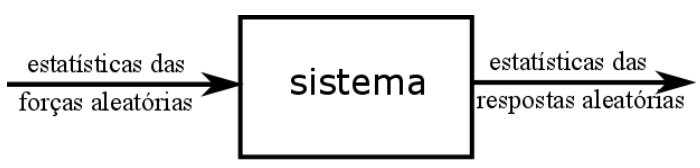

Figura 3.1: Relação entre as estatísticas das forças e respostas do sistema.

Usualmente, durante os testes modais de sistemas em condições operacionais, apenas os sinais de saída (resposta do sistema) podem ser medidos. Por isso, a terceira seção deste capítulo visa decompor as funções de correlação e densidade espectral de potência (PSD) dos sinais de resposta em relação aos parâmetros modais. Isso no entanto só é possível perante uma das seguintes hipóteses: o sinal de forçamento poder ser modelado como um ruído branco 
ou as respostas do sistema em coordenadas modais não serem correlacionadas. Para essas duas hipóteses, a identificação do sistema passa a ser realizada através de um ajuste de curvas das funções de correlação e PSDs.

Durante o desenvolvimento deste capítulo, utiliza-se também a hipótese dos sinais serem sempre considerados como processos fracamente estacionários. Isso significa que as médias e variâncias do processo são constantes, isto é, independente do instante de tempo.

\section{1}

\section{Função de correlação e densidade espectral de potência}

Seja $\mathbf{p}(t)$ e $\mathbf{s}(t), t \in \mathbb{R}$, dois processos estocásticos compostos por vetores aleatórios de dimensões $\mathbb{R}^{n p}$ e $\mathbb{R}^{n s}$, respectivamente. As funções de correlação entres esses dois processos (correlação cruzada), em instantes de tempo $t_{1}$ e $t_{2}$, são definidas como sendo

$$
\mathbf{R}_{p s}\left(t_{1}, t_{2}\right)=\mathbf{E}\left[\mathbf{p}\left(t_{1}\right) \mathbf{s}\left(t_{2}\right)^{T}\right],
$$

onde $\mathbf{R}_{p s}\left(t_{1}, t_{2}\right)$ é a matriz de correlação dos processos e tem dimensão $n p \times n s$. Cada elemento da matriz corresponde a correlação entre uma componente de $\mathbf{p}(t)$ em $t=t_{1}$ com outra de $\mathbf{s}(t)$ em $t=t_{2}$. Para o caso particular dos processos serem estacionários, a correlação deixa de ser uma função dos dois instantes de tempo e passa a ser função apenas da diferença entre eles, isto é, $\mathbf{R}_{p s}\left(t_{1}, t_{2}\right) \rightarrow \mathbf{R}_{p s}\left(t_{2}-t_{1}\right)$. Definindo a variável $\tau=t_{2}-t_{1}$, a correlação entre dois processos estacionários é dado pela equação

$$
\mathbf{R}_{p s}(\tau)=\mathbf{E}\left[\mathbf{p}(t) \mathbf{s}^{T}(t+\tau)\right] .
$$

Para cada valor de $\tau, \mathbf{R}_{p s}(\tau)$ corresponde a uma matriz de dimensão $n p \times n s$. Na prática, o cálculo das correlações pela Eq. (3-2) é impossível de ser realizado já que os processos só podem ser avaliados em um intervalo de tempo finito. Para sinais ergódicos, é possível então definir um estimador para as correlações, utilizando apenas um intervalo $T$ de observações. Em um sinal ergódico, as estatísticas das variáveis aleatórias em cada instante são iguais (visto que um processo ergódico é estacionário) e podem ser calculadas através de uma longa realização ao longo do tempo. O tamanho do intervalo de tempo utilizado pelo estimador deve ser grande o suficiente para que as funções de correlação tenham atingido uma convergência em seus valores. Esse estimador é definido como sendo

$$
\hat{\mathbf{R}}_{p s}(\tau)=\frac{1}{T} \int_{0}^{T} \mathbf{p}(t) \mathbf{s}^{T}(t+\tau) d t
$$

Uma importante vantagem desse estimador é o fato dele poder ser escrito através do operador de convolução. Para isso, é preciso transformar a variável 
de integração para $\theta=t+\tau$, resultando em

$$
\hat{\mathbf{R}}_{p s}(\tau)=\frac{1}{T} \int_{-T-\tau}^{-\tau} \mathbf{p}(-\theta-\tau) \mathbf{s}^{T}(-\theta) d \theta .
$$

Utilizando a premissa de estacionalidade dos processos e supondo que seus valores também são conhecidos fora do intervalo $t \in[0 ; T]$, pode-se transladar os limites de integração em um valor de $T+\tau$, resultando em

$$
\hat{\mathbf{R}}_{p s}(\tau)=\frac{1}{T} \int_{0}^{T} \mathbf{p}(-\theta-\tau) \mathbf{s}^{T}(-\theta) d \theta .
$$

Pode-se mostrar que a integral acima é uma convolução quando $T \rightarrow \infty$. A Eq. (3-5) pode então ser reescrita como

$$
\hat{\mathbf{R}}_{p s}(\tau)=\mathbf{p}(-t) * \mathbf{s}^{T}(t) .
$$

Para processos estacionários, a função densidade espectral de potência $\mathbf{G}_{p s}(\omega)$ é definida como sendo a transformada de Fourier da função de correlação [31], ou seja

$$
\mathbf{G}_{p s}(\omega)=\frac{1}{2 \pi} \int_{-\infty}^{\infty} \mathbf{R}_{p s}(\tau) e^{-i \omega \tau} d \tau
$$

A correlação pode ser definida como a transformada de Fourier inversa da densidade espectral de potência,

$$
\mathbf{R}_{p s}(\tau)=\int_{-\infty}^{\infty} \mathbf{G}_{p s}(\omega) e^{i \omega \tau} d \omega
$$

O par de Eqs. (3-7/3-8) forma a relação chamada de Wiener-Khinchine. Observe que tanto as funções de correlação quando as PSD contém a mesma quantidade de informações sobre o sinal. A única diferença entre elas é o domínio em são definidas.

Vale ressaltar que, caso o estimador para as funções de correlação definido pela Eq. (3-6) seja transformado para o domínio da frequência, o resultado será um estimador para a densidade espectral de potência. Assim, a PSD pode ser estimada através de

$$
\hat{\mathbf{G}}_{p s}(\omega)=\mathbf{P}^{*}(\omega) \mathbf{S}^{T}(\omega)
$$

\section{2}

\section{Relação entre forçamento e resposta do sistema}

Neste capítulo será apresentado a relação entre as estatísticas dos sinais de entrada e saída para sistemas lineares com múltiplos graus de liberdade. O caso de um grau de liberdade passa a ser então um caso particular. Alguns dos resultados apresentados no capítulo anterior serão utilizados ao longo das próximas seções.

Nas seções 2.2.3 e 2.2.4, a resposta no tempo para um sistema qualquer 
pôde ser escrita como um somatório das convoluções entre as respectivas respostas ao impulso e o forçamento. Isso foi mostrado na Eq.(2-75) e é repetido aqui por conveniência

$$
\mathbf{q}(t)=\mathbf{H}(t) * \mathbf{u}(t) .
$$

Ao calcular a transposta dessa equação, obtêm-se como resultado

$$
\mathbf{q}^{T}(t)=\mathbf{u}^{T}(t) * \mathbf{H}^{T}(t) .
$$

Utilizando a hipótese de que o forçamento $\mathbf{u}(t)$ é agora um processo estocástico fracamente estacionário, a resposta do sistema $\mathbf{q}(t)$ também passa a ser um processo do mesmo tipo. A autocorrelação da resposta do sistema pode ser calculada através da Eq. (3-2), resultando em

$$
\mathbf{R}_{q q}(t)=\mathbf{E}\left[\mathbf{q}(t) \mathbf{q}^{T}(t+\tau)\right] .
$$

Substituindo $\mathbf{q}^{T}(t)$ como definido na Eq. (3-11), obtêm-se

$$
\begin{aligned}
\mathbf{R}_{q q}(t) & =\mathbf{E}\left[\mathbf{q}(t)\left\{\mathbf{u}^{T}(t+\tau) * \mathbf{H}^{T}(t)\right\}\right] \\
& =\mathbf{E}\left[\mathbf{q}(t)\left\{\int_{-\infty}^{\infty} \mathbf{u}^{T}(t+\tau-\alpha) \mathbf{H}^{T}(\alpha) d \alpha\right\}\right] \\
& =\int_{-\infty}^{\infty} \mathbf{E}\left[\mathbf{q}(t) \mathbf{u}^{T}(t+\tau-\alpha)\right] \mathbf{H}^{T}(\alpha) d \alpha
\end{aligned}
$$

Como o termo $\mathbf{E}\left[\mathbf{q}(t) \mathbf{u}^{T}(t+\tau-\alpha)\right]$ é a correlação cruzada entre a resposta e o forçamento, ou seja, $\mathbf{R}_{q u}(\tau-\alpha)$, a Eq. (3-13) pode ser simplificada para

$$
\mathbf{R}_{q q}(t)=\int_{-\infty}^{\infty} \mathbf{R}_{q u}(\tau-\alpha) \mathbf{H}^{T}(\alpha) d \alpha=\mathbf{R}_{q u}(\tau) * \mathbf{H}^{T}(\tau)
$$

Utilizando a expressão da resposta do sistema definido na Eq. (3-10), o termo da correlação cruzada $\mathbf{R}_{q u}(\tau)$ pode ser escrito como

$$
\begin{aligned}
\mathbf{R}_{q u}(\tau) & =\mathbf{E}\left[\mathbf{q}(t) \mathbf{u}^{T}(t+\tau)\right] \\
& =\mathbf{E}\left[\left\{\int_{-\infty}^{\infty} \mathbf{H}(\alpha) \mathbf{u}(t-\alpha) d \alpha\right\} \mathbf{u}^{T}(t+\tau)\right] \\
& =\int_{-\infty}^{\infty} \mathbf{H}(\alpha) \mathbf{E}\left[\mathbf{u}(t-\alpha) \mathbf{u}^{T}(t+\tau)\right] d \alpha \\
& =\int_{-\infty}^{\infty} \mathbf{H}(\alpha) \mathbf{R}_{u u}(\tau+\alpha) d \alpha
\end{aligned}
$$

Propondo uma mudança na variável de integração na forma $\beta=-\alpha$, a Eq. (315) se torna

$$
\mathbf{R}_{q u}(\tau)=\int_{-\infty}^{\infty} \mathbf{H}(-\beta) \mathbf{R}_{u u}(\tau-\beta) d \beta=\mathbf{H}(-\tau) * \mathbf{R}_{u u}(\tau)
$$

Substituindo o resultado da Eq. (3-16) na Eq. (3-14), a seguinte relação entre as correlações do forçamento e resposta (sinais de entrada e saída) é obtida 


$$
\mathbf{R}_{q q}(\tau)=\mathbf{H}(-\tau) * \mathbf{R}_{u u}(\tau) * \mathbf{H}^{T}(\tau)
$$

Ao transformar essa relação para o domínio da frequência através da transformada de Fourier, obtêm-se a relação entre as PSD

$$
\mathbf{G}_{q q}(\omega)=\tilde{\mathbf{H}}^{*}(\omega) \mathbf{G}_{u u}(\omega) \tilde{\mathbf{H}}(\omega)
$$

O par de equações (3-17/3-18) constitui um resultado clássico em vibrações aleatórias. Essas equações relacionam as estatísticas dos sinais de entrada com os de saída do sistema, tanto no domínio do tempo quanto no domínio da frequência.

\section{3}

\section{Decomposição modal}

Em análise modal operacional, apenas as respostas do sistema a um forçamento aleatório são conhecidas. As forças que atuam no sistema são modeladas como processos estocásticos e devem atender algumas restrições, porém não precisam ser medidas. Como só a resposta do sistema é conhecida, as funções de resposta ao impulso unitário e as funções de resposta em frequência não podem ser calculadas experimentalmente. A identificação do sistema só é então possível caso alguma função gerada pelas respostas do sistema possa ser decomposta em relação aos parâmetros modais. Perante algumas hipóteses, as funções de correlação e de densidade espectrais de potência podem sofrer esse tipo de decomposição. Para isso, as hipóteses do sistema ser forçado por um ruído branco ou das coordenadas modais serem não correlacionadas são necessárias. A decomposição utilizando cada uma dessas hipóteses é mostrada nas próximas seções.

\subsection{1}

\section{Hipótese de forçamento por ruído branco}

Antes de realizar as decomposições modais mencionadas acima, é preciso apresentar a definição de ruído branco. Um ruído branco é um processo estocástico ergódico tal que sua densidade espectral é uma função constante em todo domínio da frequência. Sendo o forçamento $\mathbf{u}(t)$ um vetor composto de $n$ ruídos brancos, sua matriz de PSD é

$$
\mathbf{G}_{u u}(\omega)=\mathbf{G}_{0}, \quad-\infty<\omega<\infty,
$$

onde $\mathbf{G}_{0}$ é uma matriz constante e com dimensão $n \times n$. Caso os ruídos brancos no vetor $\mathbf{u}(t)$ não forem correlacionados entre si, a matriz $\mathbf{G}_{0}$ se torna diagonal. Ao substituir a PSD do ruído branco na Eq. (3-8), obtêm-se as funções de 
correlação na forma

$$
\begin{aligned}
\mathbf{R}_{u u}(\tau) & =\int_{-\infty}^{\infty} \mathbf{G}_{0} e^{i \omega \tau} d \omega \\
& =2 \pi \mathbf{G}_{0} \delta(\tau)
\end{aligned}
$$

onde $\delta(t)$ é a função delta de Dirac.

Pelo do teorema de Parseval [32], não é possível obter uma realização de um ruído branco propriamente dito. Isso porque a variância do sinal (integral da PSD em todo o domínio) tenderia a infinito, levando o processo a ter energia infinita. Uma forma de contornar esse problema é aproximar um ruído branco por um processo estocástico com PSD constante em uma banda de frequência e zero fora dela. Experimentalmente, tem-se como frequência limite da banda a frequência de Nyquist, que corresponde a metade da frequência de aquisição utilizada.

Suponha que $u_{i}(t)$ seja uma componente do vetor $\mathbf{u}(t)$. Define-se sua PSD como sendo constantes e igual a $G_{u 0}{ }^{1}$ no intervalo de frequência $\left(-f_{c}<f<f_{c}\right)$, onde $f_{c}$ é a frequência de corte do espectro, e zero fora desse intervalo. $\mathrm{O}$ teorema de Parseval mostra então que a variância deste processo é

$$
\sigma_{u}^{2}=2 G_{u 0} f_{c}
$$

Observe que a constante da densidade espectral é relacionado tanto com variância do sinal tanto com frequência de corte. Ela pode ser escrita como

$$
G_{u 0}=\frac{\sigma_{u}^{2}}{2 f_{c}}
$$

A correlação deste sinal pode ser aproximada por uma função delta quando a frequência de corte é relativamente alta, resultando em

$$
R_{u u}(\tau)=2 \pi \frac{\delta_{u}^{2}}{2 f_{c}} \delta(\tau)
$$

Quando utilizado todos os componentes do vetor $\mathbf{u}(t)$, as Eqs. (3-22/3-23) se transformam em

$$
\mathbf{R}_{u u}(\tau)=\pi \frac{\mathbf{C}_{u}}{f_{c}} \delta(\tau) ; \quad \mathbf{G}_{u u}(\omega)=\left\{\begin{array}{l}
\frac{\mathbf{C}_{u}}{2 f_{c}}, \text { para }|\omega| \leq f_{c} \\
0, \text { para }|\omega|>f_{c}
\end{array}\right.
$$

onde a matriz $\mathbf{C}_{u}$ é a matriz de covariância dos ruídos brancos. Duas importantes características podem ser observadas na Eq. (3-24). A primeira é que as funções de correlação em $\mathbf{R}_{u u}(\tau)$ são correlacionadas apenas em $\tau=0$. A segunda é que as PSDs em $\mathbf{G}_{u u}$ têm um valor constante em uma banda de frequência. Essas duas características serão utilizadas na decomposição modal

${ }^{1}$ Embora definido por uma letra maiúscula, esse termo é um escalar. 
após o exemplo.

\section{Exemplo 3.1}

Seja u(t) um ruído branco com média zero e variância igual a 100. Este processo corresponde ao vetor $\mathbf{u}(t)$ quando $n=1$. Uma longa realização do sinal foi feita com uma frequência de aquisição de $1 \mathrm{kHz}$ por 100 segundo, gerando um total de $10^{5}$ amostras. A frequência de corte para a estimativa da PSD é a frequência de Nyquist, que corresponde a $500 \mathrm{~Hz}$. A estimativa para a função de correlação e densidade espectral do processo foi realizada através do método de Welch, que segmenta a longa realização em pequenos blocos. Mais informações sobre o método é encontrado em [26]. Os resultados são mostrados na Figura 3.2. O gráfico no topo, mostra uma parte da longa realização de u(t). O gráfico no canto inferior direito mostra a estimativa da PSD, e como esperado, é uma função aproximadamente constante e com amplitude igual a $\frac{\sigma_{u}^{2}}{2 f_{c}}$ dentro da banda de frequência $(-500 \mathrm{~Hz}, 500 \mathrm{~Hz})$. Já gráfico do canto inferior esquerdo mostra a estimativa da função de correlação, e como esperado, tem o formato de um impulso unitário. Como estamos tratando de sinais discretos, a amplitude do pico não é infinito e sim 100, que corresponde a variância do sinal. (Fim do exemplo)
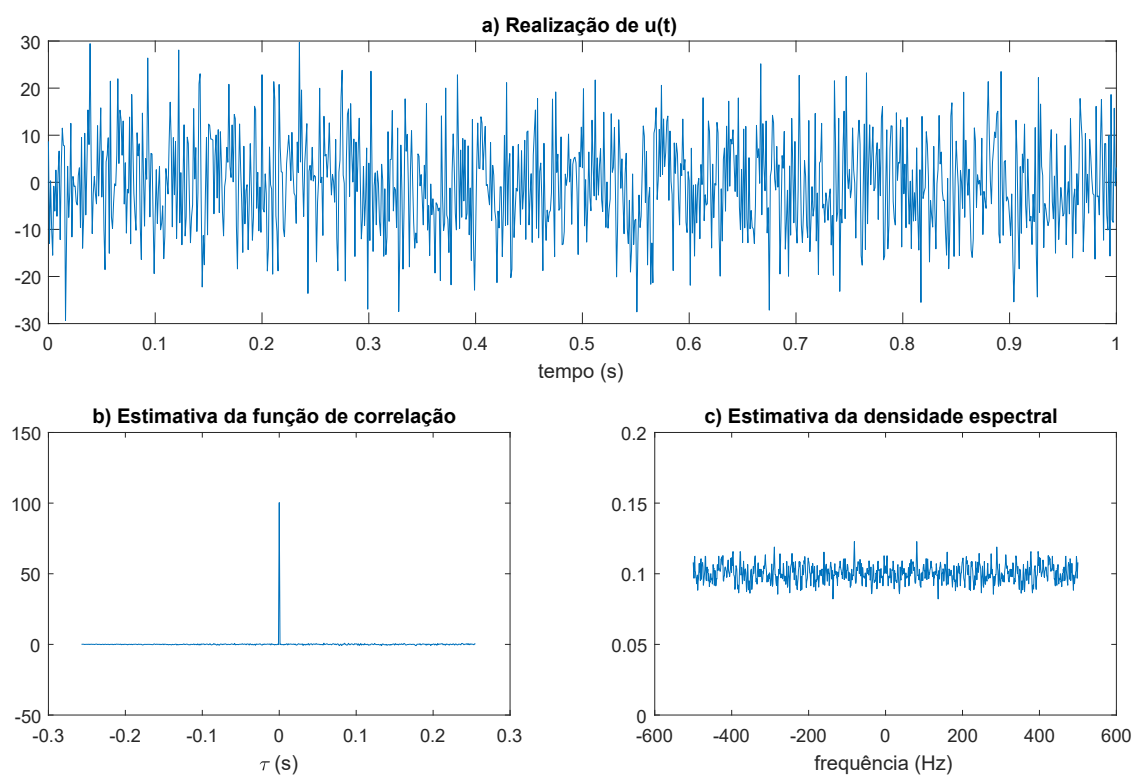

Figura 3.2: a) Realização do sinal $u(t)$. b) Estimativa da função de correlação. c) Estimativa da densidade espectral de potência.

Uma vez tendo definido o ruído branco e suas principais características, é possível agora estudar a resposta do sistema quando submetido a esse tipo de 
forçamento. O objetivo é descrever as funções de correlação e densidade espectral em relação aos parâmetros modais, realizando então uma decomposição modal. [33][34][35][36]

Sabendo que as FRFs definidas no capítulo anterior têm a propriedade de $\mathbf{H}^{*}(\omega)=\mathbf{H}(-\omega)$, a equação fundamental definida pela Eq. (3-18) pode ser reescrita como

$$
\mathbf{G}_{q q}(\omega)=\mathbf{H}(-\omega) \mathbf{G}_{u u}(\omega) \mathbf{H}(\omega) .
$$

No capítulo anterior, as FRFs dos sistema foram definidas para duas modelagens distintas: uma para o sistema com amortecimento proporcional, Eq. (2-70), e outra para o sistema com amortecimento viscoso generalizado, Eq. (2-102). Ambas equações têm mesma estrutura, o que torna indiferente o tipo de modelo que será utilizado a seguir. Substituindo uma dessas duas definições na Eq. (3-25), obtêm-se como resultado

$$
\begin{aligned}
\mathbf{G}_{q q}(\omega)= & {\left[\sum_{r=1}^{n}\left(\frac{\mathbf{A}_{r}}{-i \omega-\lambda_{r}}+\frac{\mathbf{A}_{r}^{*}}{-i \omega-\lambda_{r}^{*}}\right)\right] \mathbf{G}_{u u}\left[\sum_{r=1}^{n}\left(\frac{\mathbf{A}_{r}}{i \omega-\lambda_{r}}+\frac{\mathbf{A}_{r}^{*}}{i \omega-\lambda_{r}^{*}}\right)\right] } \\
= & \sum_{r=1}^{n} \sum_{s=1}^{n}\left(\frac{\mathbf{A}_{r}}{-i \omega-\lambda_{r}}+\frac{\mathbf{A}_{r}^{*}}{-i \omega-\lambda_{r}^{*}}\right) \mathbf{G}_{u u}\left(\frac{\mathbf{A}_{s}}{i \omega-\lambda_{s}}+\frac{\mathbf{A}_{s}^{*}}{i \omega-\lambda_{s}^{*}}\right) \\
= & \sum_{r=1}^{n} \sum_{s=1}^{n}\left(\frac{\mathbf{A}_{r} \mathbf{G}_{u u} \mathbf{A}_{s}}{\left(-i \omega-\lambda_{r}\right)\left(i \omega-\lambda_{s}\right)}+\frac{\mathbf{A}_{r} \mathbf{G}_{u u} \mathbf{A}_{s}^{*}}{\left(-i \omega-\lambda_{r}\right)\left(i \omega-\lambda_{s}^{*}\right)}+\right. \\
& \left.+\frac{\mathbf{A}_{r}^{*} \mathbf{G}_{u u} \mathbf{A}_{s}}{\left(-i \omega-\lambda_{r}^{*}\right)\left(i \omega-\lambda_{s}\right)}+\frac{\mathbf{A}_{r}^{*} \mathbf{G}_{u u} \mathbf{A}_{s}^{*}}{\left(-i \omega-\lambda_{r}^{*}\right)\left(i \omega-\lambda_{s}^{*}\right)}\right) .
\end{aligned}
$$

Para cada termo na Eq. (3-26), pode-se utilizar o método de Heaviside para calcular suas respectivas frações parciais. Os resultados para cada um dos termos são

$$
\begin{aligned}
\frac{\mathbf{A}_{r} \mathbf{G}_{u u} \mathbf{A}_{s}}{\left(-i \omega-\lambda_{r}\right)\left(i \omega-\lambda_{s}\right)} & =\frac{\mathbf{A}_{r} \mathbf{G}_{u u} \mathbf{A}_{s}}{-\lambda_{r}-\lambda_{s}}\left(\frac{1}{-i \omega-\lambda_{r}}+\frac{1}{i \omega-\lambda_{s}}\right) \\
\frac{\mathbf{A}_{r} \mathbf{G}_{u u} \mathbf{A}_{s}^{*}}{\left(-i \omega-\lambda_{r}\right)\left(i \omega-\lambda_{s}^{*}\right)} & =\frac{\mathbf{A}_{r} \mathbf{G}_{u u} \mathbf{A}_{s}^{*}}{-\lambda_{r}-\lambda_{s}^{*}}\left(\frac{1}{-i \omega-\lambda_{r}}+\frac{1}{i \omega-\lambda_{s}^{*}}\right) \\
\frac{\mathbf{A}_{r}^{*} \mathbf{G}_{u u} \mathbf{A}_{s}}{\left(-i \omega-\lambda_{r}^{*}\right)\left(i \omega-\lambda_{s}\right)} & =\frac{\mathbf{A}_{r}^{*} \mathbf{G}_{u u} \mathbf{A}_{s}}{-\lambda_{r}^{*}-\lambda_{s}}\left(\frac{1}{-i \omega-\lambda_{r}^{*}}+\frac{1}{i \omega-\lambda_{s}}\right) \\
\frac{\mathbf{A}_{r}^{*} \mathbf{G}_{u u} \mathbf{A}_{s}^{*}}{\left(-i \omega-\lambda_{r}^{*}\right)\left(i \omega-\lambda_{s}^{*}\right)} & =\frac{\mathbf{A}_{r}^{*} \mathbf{G}_{u u} \mathbf{A}_{s}^{*}}{-\lambda_{r}^{*}-\lambda_{s}^{*}}\left(\frac{1}{-i \omega-\lambda_{r}^{*}}+\frac{1}{i \omega-\lambda_{s}^{*}}\right)
\end{aligned}
$$


Substituindo essas frações parciais na Eq. (3-26), obtêm-se

$$
\begin{aligned}
\mathbf{G}_{q q}(\omega)=\sum_{r=1}^{n} \sum_{s=1}^{n} & \left(\left(\frac{\mathbf{A}_{r} \mathbf{G}_{u u} \mathbf{A}_{s}}{-\lambda_{r}-\lambda_{s}}+\frac{\mathbf{A}_{r} \mathbf{G}_{u u} \mathbf{A}_{s}^{*}}{-\lambda_{r}-\lambda_{s}^{*}}\right) \frac{1}{-i \omega-\lambda_{r}}+\right. \\
+ & \left(\frac{\mathbf{A}_{r}^{*} \mathbf{G}_{u u} \mathbf{A}_{s}}{-\lambda_{r}^{*}-\lambda_{s}}+\frac{\mathbf{A}_{r}^{*} \mathbf{G}_{u u} \mathbf{A}_{s}^{*}}{-\lambda_{r}^{*}-\lambda_{s}^{*}}\right) \frac{1}{-i \omega-\lambda_{r}^{*}}+ \\
& +\left(\frac{\mathbf{A}_{r} \mathbf{G}_{u u} \mathbf{A}_{s}}{-\lambda_{r}-\lambda_{s}}+\frac{\mathbf{A}_{r}^{*} \mathbf{G}_{u u} \mathbf{A}_{s}}{-\lambda_{r}^{*}-\lambda_{s}}\right) \frac{1}{i \omega-\lambda_{s}}+ \\
& \left.+\left(\frac{\mathbf{A}_{r} \mathbf{G}_{u u} \mathbf{A}_{s}^{*}}{-\lambda_{r}-\lambda_{s}^{*}}+\frac{\mathbf{A}_{r}^{*} \mathbf{G}_{u u} \mathbf{A}_{s}}{-\lambda_{r}^{*}-\lambda_{s}^{*}}\right) \frac{1}{i \omega-\lambda_{s}^{*}}\right)
\end{aligned}
$$

Uma matriz $\mathbf{B}_{r}$ pode então ser definida como a soma ponderada das matrizes residuais,

$$
\mathbf{B}_{r}=\sum_{s=1}^{n}\left(\frac{\mathbf{A}_{s}}{-\lambda_{r}-\lambda_{s}}+\frac{\mathbf{A}_{s}^{*}}{-\lambda_{r}-\lambda_{s}^{*}}\right), \quad r=1, \ldots, n .
$$

Substituindo essas matrizes na Eq. (3-28), pode-se reescrever as PSDs decomposta em relação aos parâmetros modais, isto é,

$$
\mathbf{G}_{q q}(\omega)=\sum_{r=1}^{n}\left(\frac{\mathbf{A}_{r} \mathbf{G}_{u u} \mathbf{B}_{r}}{-i \omega-\lambda_{r}}+\frac{\mathbf{A}_{r}^{*} \mathbf{G}_{u u} \mathbf{B}_{r}^{*}}{-i \omega-\lambda_{r}^{*}}+\frac{\mathbf{B}_{r} \mathbf{G}_{u u} \mathbf{A}_{r}}{i \omega-\lambda_{r}}+\frac{\mathbf{B}_{r}^{*} \mathbf{G}_{u u} \mathbf{A}_{r}^{*}}{i \omega-\lambda_{r}^{*}}\right)
$$

A Eq. (3-30) pode ser ainda reescrita na forma residual. Para isso, é necessário que vetores $\gamma_{r}$ seja definido. Esses vetores são chamados de vetores de participação modal e são definidos como

$$
\gamma_{r}=\mathbf{B}_{r} \mathbf{G}_{u u} \frac{\boldsymbol{\psi}_{r}}{a_{r}}, \quad r=1, \ldots, n .
$$

Como o nome já diz, esses vetores são responsáveis por quantificar a contribuição que cada modo nas respostas do sistema. Como pode ser verificado na Eq. (3-31), esses vetores de contribuição são função das PSDs dos forçamento, que são constantes no caso do ruído branco. Lembrando que as matrizes $\mathbf{A}_{r}$ são iguais a $\frac{\psi_{r} \psi_{r}^{T}}{a_{r}}$, os quatro termos da Eq. (3-30) podem ser reescritos utilizando o vetor de participação modal, resultando em

$$
\mathbf{G}_{q q}=\sum_{r=1}^{n}\left(\frac{\boldsymbol{\psi}_{r} \gamma_{r}^{T}}{-i \omega-\lambda_{r}}+\frac{\boldsymbol{\psi}_{r}^{*} \gamma_{r}^{H}}{-i \omega-\lambda_{r}^{*}}+\frac{\boldsymbol{\gamma}_{r} \boldsymbol{\psi}_{r}^{T}}{i \omega-\lambda_{r}}+\frac{\boldsymbol{\gamma}_{r}^{*} \boldsymbol{\psi}_{r}^{H}}{i \omega-\lambda_{r}^{*}}\right),
$$

onde $\square^{H}$ corresponde ao transposto do complexo conjugado.

Pelas Eqs. (3-30/3-32), pode-se observar que, dos quatro termos presentes no somatório, dois são dominantes próximo das frequência naturais positivas e dois próximo das frequência naturais negativas. O segundo termo dessas equações é dominantes próximo das frequência naturais positivas, visto que o denominador $-i \omega-\lambda_{n}^{*}$ é igual a $-i \omega+i \omega_{d r}+\zeta_{r} \omega_{n r}$, e por isso se torna pequeno quando $\omega \rightarrow \omega_{d r}$. O terceiro termo também é dominante próximo 
das frequência naturais positivas, já que o denominador $i \omega-\lambda_{r}$ é igual a $i \omega-i \omega_{d r}+\zeta_{r} \omega_{n r}$ e também se torna pequeno quando $\omega \rightarrow \omega_{d r}$. O primeiro e quarto termo são dominantes próximo das frequência naturais negativas. Isso pode ser explicado de forma semelhante ao que foi feito para aos outros dois termos.

Utilizando a relação de Wiener-Khinchine definida pelas Eqs. (3-7/38), as funções de correlação podem ser definidas através da transformada de Fourier. No entanto, como mostrado em [27], é necessário que as funções de correlação sejam divididas em duas expressões distintas, sendo uma para $\tau<0$ e outra para $\tau>0$. Assim, a relação de Wiener-Khinchine se transforma em

$$
\mathbf{G}_{q q}=\frac{1}{\pi} \int_{-\infty}^{0} \mathbf{R}_{q q-}(\tau) e^{-i \omega \tau} d \tau+\frac{1}{\pi} \int_{0}^{\infty} \mathbf{R}_{q q+}(\tau) e^{-i \omega \tau} d \tau
$$

Utilizando a expressão da densidade espectral definida pela Eq. (3-32) e a transformada de Fourier acima, pode-se mostrar que as funções de correlação são iguais a

$$
\begin{gathered}
\mathbf{R}_{q q-}=2 \pi \sum_{r=1}^{n}\left(\boldsymbol{\psi}_{r} \boldsymbol{\gamma}_{r}^{T} e^{-\lambda_{r} \tau}+\boldsymbol{\psi}_{r}^{*} \boldsymbol{\gamma}_{r}^{H} e^{-\lambda_{r}^{*} \tau}\right), \text { para } \tau \leq 0 \\
\mathbf{R}_{q q+}=2 \pi \sum_{r=1}^{n}\left(\boldsymbol{\gamma}_{r} \boldsymbol{\psi}_{r}^{T} e^{\lambda_{r} \tau}+\boldsymbol{\gamma}_{r}^{*} \boldsymbol{\psi}_{r}^{H} e^{\lambda_{r}^{*} \tau}\right), \text { para } \tau \geq 0
\end{gathered}
$$

O par de equações (3-34/3-35) é o principal resultado deste capítulo porque demonstra o formato de decaimento exponencial das funções de correlação. Além disso, elas mostram a decomposição modal necessária para os métodos de identificação. Ao comparar essas funções com as de responsa ao impulso (Eq. (2-74/2-104)), percebe-se que ambas têm mesma estrutura. Logo, os métodos de identificação de parâmetros utilizados na análise modal experimental podem ser aplicados diretamente na análise modal operacional. Para isso, basta substituir as funções de resposta ao impulso pelas funções de correlação. Além disso, as Eqs. (3-34/3-35) mostram a importante propriedade de simetria das funções de correlação, isto é, $\mathbf{R}(-\tau)=\mathbf{R}^{T}(\tau)$. Percebe-se também o fato de que o valor máximo das correlações ocorre quando $\tau=0$.

\section{Exemplo 3.2}

Para exemplificar a decomposição modal da PSD da resposta de um sistema excitado por ruídos brancos, utiliza-se a seguir o sistema do Exemplo 2.2. Considere que cada grau de liberdade é exitado por ruídos brancos nãocorrelacionados entre si, com distribuição Gaussiana, média zero e desvio padrão igual a 100 N. Assim, a matriz de covariância desse forçamento passa a ser 


$$
\mathbf{C}_{u 0}=100^{2} \times\left[\begin{array}{cccc}
1 & 0 & 0 & 0 \\
0 & 1 & 0 & 0 \\
0 & 0 & 1 & 0 \\
0 & 0 & 0 & 1
\end{array}\right]
$$

Sendo a frequência de amostragem utilizada para gerar as realizações dos ruídos brancos igual a $f_{s}=50 \mathrm{~Hz}$, a PSD do forçamento é calculada conforme a Eq. (3-24), onde a frequência de Nyquist foi adotada como a frequência de corte $\left(f_{c}=25 \mathrm{~Hz}\right)$. Assim, o resultado obtido é

$$
\mathbf{G}_{u u}(\omega)=\left[\begin{array}{cccc}
400 & 0 & 0 & 0 \\
0 & 400 & 0 & 0 \\
0 & 0 & 400 & 0 \\
0 & 0 & 0 & 400
\end{array}\right]
$$

Utilizando as frequências naturais e os fatores de amortecimentos listados na Tabela 2.2 e os modos normais obtidos no Exemplo 2.1, os polos $\lambda_{r} e$ as matrizes residuais $\mathbf{A}_{r}$ podem ser calculadas através das Eqs. (2-67/2-69), respectivamente. Uma vez que todos esses termos tenham sido calculados, as matrizes $\mathbf{B}_{r}$ podem ser calculadas pela Eq. (3-29). Finalmente, as PSDs do sistema podem ser calculadas através dos parâmetros modais utilizando a Eq. (3-30).

A Figura 3.3 mostra o resultado obtido para a PDS do primeiro grau de liberdade (primeira linha e coluna da matriz $\mathbf{G}_{q q}(\omega)$ ). Na mesma figura, a contribuição de cada modo também é mostrada. Assim como para as FRFs, as PSDs também apresentam um modo dominante próximo as respectivas frequências naturais.

Decomposição modal da função $G_{q q, 11}$

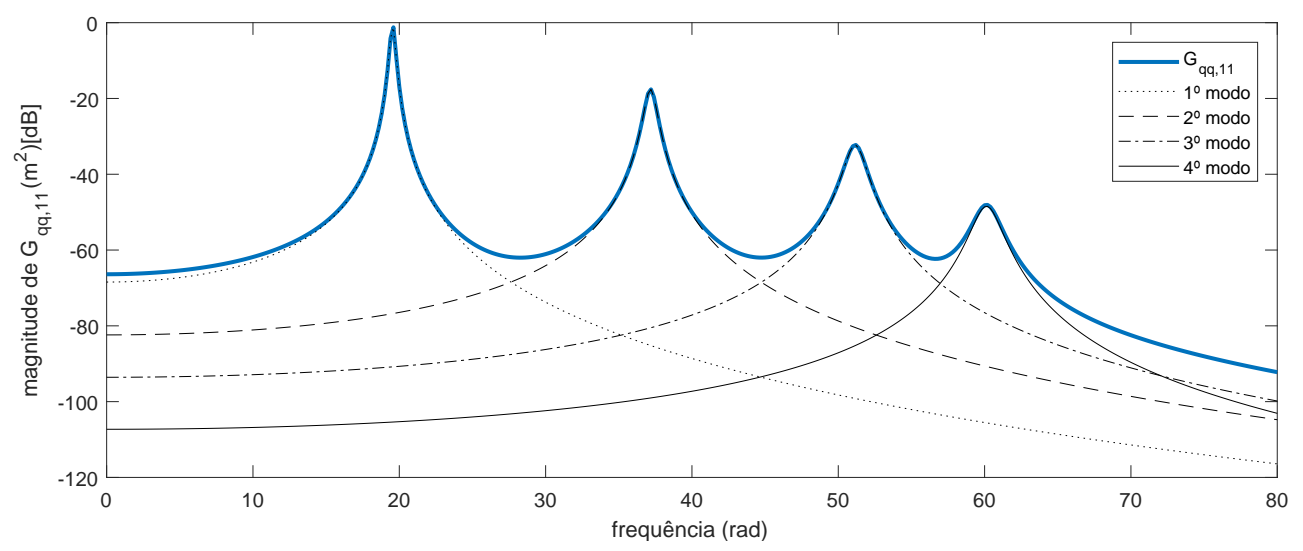

Figura 3.3: Exemplo da decomposição modal de uma PDS do sistema excitado por ruído branco.

Para validar o resultado obtido pela decomposição modal, a mesma PSD 
pode ser estimada diretamente do sinal de resposta do sistema. Para isso, uma longa realização de $\mathbf{u}(t)$ foi feita utilizando $N_{s}=2^{18}$ amostras. A partir desse forçamento, o sinal da resposta $\mathbf{q}(t)$ pode ser obtido através de uma simulação. Segmentando essa longa resposta do sistema em pequenos blocos, a matriz de densidade espectral $\mathbf{G}_{q q}$ pode ser estimada utilizando o método de Welch. $O$ resultado é mostrado pela curva vermelha na Figura 3.4. Percebe-se que nesta figura ambas as funções são iguais, validando assim a decomposição modal apresentada pelas Eqs. (3-30/3-32). (Fim do exemplo)

Validação da decomposição modas da PSD

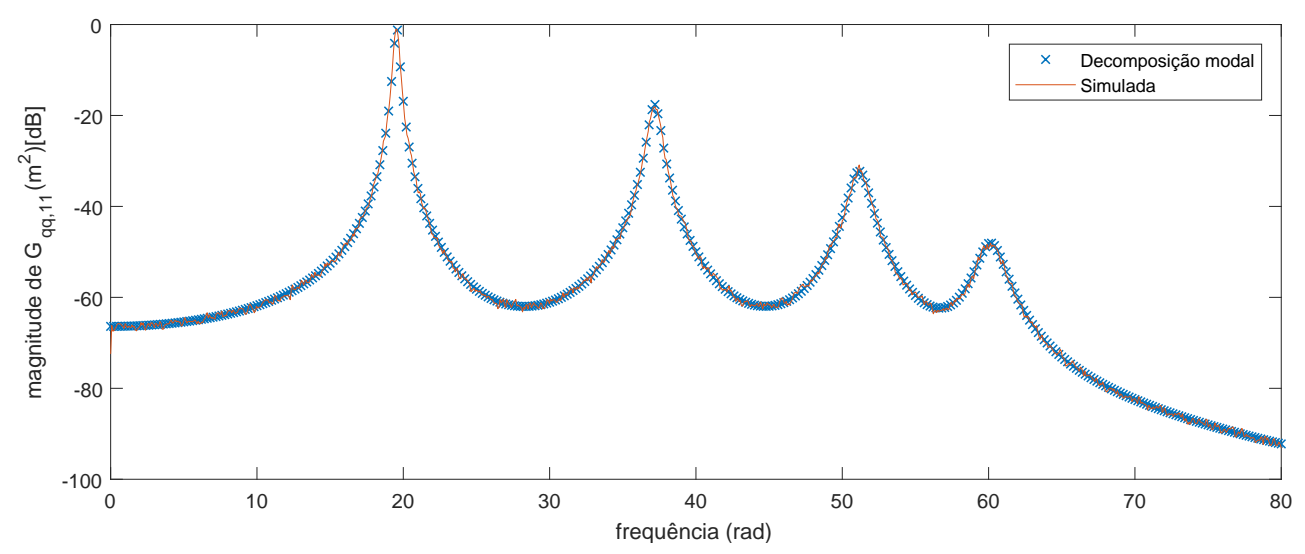

Figura 3.4: Comparação entre a PDS da resposta do sistema calculada pelos parâmetros modais do sistema com a PSD calculada diretamente pelos sinais de resposta.

\subsection{2}

\section{Hipótese de coordenadas modais não correlacionadas}

Quando a hipótese do sistema ser forçado por um ruído branco não é válida, existe uma segunda forma de decompor as funções de correlação e as PSDs em relação aos parâmetros modais. No entanto, essa alternativa exige uma outra hipótese, a das respostas do sistema serem não correlacionadas quando escritas em coordenadas modais. Como mostrado a seguir, isso é aproximadamente verdade para sistemas que apresentam baixo amortecimento ou modos bem separados (frequências naturais relativamente distantes uma da outra). Isso pode ser verificado através das matrizes de correlação e de PSD escritas em coordenadas modais, pois elas se aproximam de matrizes diagonais. Para simplificar a explicação, a hipótese do sistema ser modelado com amortecimento proporcional é utilizada.

Na seção 2.2.2, mostrou-se através das Eqs. (2-59/2-60/2-61) que os modos normais do sistema permitem o desacoplamento das equações diferenciais. Reescrevendo a Eq. (2-62) para todos os modos e de forma matricial, obtêm-se 
a equação

$$
\mathbf{z}(t)=\left[h_{r}(t)\right] * \mathbf{p}(t) .
$$

A matriz $\left[h_{r}(t)\right]$ acima corresponde a uma matriz diagonal cujos elementos são as respostas ao impulso unitário escritas com os respectivos parâmetros modais. Isto é, cada elemento da diagonal é uma função escrita na forma

$$
h_{r}(t)=\frac{e^{-\zeta_{r} \omega_{r} t}}{m_{m, r} \omega_{d, r}} \sin \omega_{d, r} t, \quad r=1, \ldots, n .
$$

Transformado a Eq. (3-36) para o domínio da frequência, têm-se como resultado a equação

$$
\tilde{\mathbf{z}}(\omega)=\left[\tilde{h}_{r}(\omega)\right] \tilde{\mathbf{p}}(\omega) .
$$

Cada componente do vetor de coordenadas modais $\tilde{\mathbf{z}}(\omega)$ pode então ser escrito, utilizando a Eq. (2-19), como

$$
\tilde{z}_{r}(\omega)=\tilde{h}_{r}(\omega) \tilde{p}_{r}(\omega)=\frac{\tilde{p}_{r}(\omega)}{m_{m, r}\left(i \omega-\lambda_{r}\right)\left(i \omega-\lambda_{r}^{*}\right)} .
$$

Novamente, o parâmetro $\lambda$ corresponde ao $r$-ésimo polo do sistema e $m_{r m}$ a $r$-ésima massa modal.

Ao utilizar o estimador da densidade espectral definido pela Eq. (3-9), a PSD da resposta do sistema em coordenadas modais pode ser obtida através da equação

$$
\mathbf{G}_{z z}(\omega)=\tilde{\mathbf{z}}^{*}(\omega) \tilde{\mathbf{z}}^{T}(\omega)
$$

Para o elemento da linha $i$ e coluna $j$ da matriz $\mathbf{G}_{z z}(\omega)$, sua respectiva densidade espectral é obtida a partir do produto $\tilde{z}_{i}^{*}(\omega) \tilde{z}_{j}(\omega)$, onde $\tilde{z}_{i}(\omega)$ e $\tilde{z}_{j}(\omega)$ são definidos pela Eq. (3-39). Quando o sistema analisado tem baixo amortecimento, cada uma das funções $\tilde{z}_{r}(\omega)$ é relevante apenas em uma estreita faixa de frequência, próxima a sua respectiva frequência natural. Quando os modos são bem separados, os valores dessas frequências são distantes, fazendo com que $\tilde{z}_{i}^{*}(\omega) \tilde{z}_{j}^{*}(\omega) \approx 0$ para $i \neq j$. Isso significa que, quando o sistema apresenta baixo amortecimento ou tem modos bem separados, a matriz com as densidades espectrais de potência é aproximadamente diagonal. Através da relação de Wiener-Khinchine, pode-se concluir então que a matriz de correlação também é aproximadamente diagonal.

Essa conclusão permite a decomposição das funções de correlação em relação a aos parâmetros modais. A resposta do sistema em coordenadas modais pode ser reescrita nas coordenadas originais através da Eq. (2-59), repetida aqui por conveniência,

$$
\mathbf{q}(t)=\mathbf{\Psi} \mathbf{z}(t)
$$

Utilizando a expressão acima, pode-se calcular a matriz de correlação da 
respostas $\mathbf{R}_{q q}$ como sendo

$$
\begin{aligned}
\mathbf{R}_{q q}(\tau) & =\mathbf{E}\left[\mathbf{q}(t) \mathbf{q}^{T}(t+\tau)\right] \\
& =\boldsymbol{\Psi} \mathbf{E}\left[\mathbf{z}(t) \mathbf{z}^{T}(t+\tau)\right] \boldsymbol{\Psi}^{T} \\
& =\boldsymbol{\Psi} \mathbf{R}_{z z}(\tau) \boldsymbol{\Psi}^{T}
\end{aligned}
$$

Utilizando a hipótese de que as coordenadas modais não são correlacionadas, a matriz $\mathbf{R}_{z z}$ é dita diagonal. Assim, pode-se reescrever a Eq. (3-42) na forma

$$
\mathbf{R}_{q q}(\tau)=\sum_{r=1}^{n} \boldsymbol{\psi}_{r} \boldsymbol{\psi}_{r}^{T} R_{z z, r}(\tau)
$$

onde as funções $R_{z z, r}(\tau)$ correspondem aos $n$ termos da diagonal de $\mathbf{R}_{z z}(\tau)$. A equação (3-43) está escrita como uma decomposição modal, visto que cada termo na somatória é expresso pelos parâmetros de um respectivo modo. Transformando a Eq. (3-43) para o domínio da frequência, a matriz de PSD também pode ser escrita de forma decomposta. O resultado é a equação

$$
\mathbf{G}_{q q}(\omega)=\sum_{r=1}^{n} \boldsymbol{\psi}_{r} \boldsymbol{\psi}_{r}^{T} G_{z z, r}(\tau)
$$

\section{Exemplo 3.3}

Este exemplo tem como objetivo ilustrar a decomposição modal pela hipótese de coordenadas modais não correlacionadas. Para isso, serão utilizados o mesmo sistema e forçamento do Exemplo 3.2 será novamente utilizado. Obtendo a resposta do deslocamento $\mathbf{q}(t)$ através de uma simulação após a longa realização do forçamento $\mathbf{u}(t)$, essa mesma resposta pôde ser transformada para coordenadas modais através da Eq. (3-41) e pelos modos normais do sistema obtidos no Exemplo 2.1. Uma vez que $\mathbf{z}(t)$ tenha sido obtido, a matriz $\mathbf{G}_{q q}(\omega)$ pode ser estimada ao dividir o longo sinal em pequenos blocos e aplicar o método de Welch [26]. Como discutido acima, essa matriz é aproximadamente diagonal. A decomposição modal das PSDs podem então ser feita pela Eq. (344). Para o sistema e forçamento deste exemplo, o resultado para a PSD do primeiro grau de liberdade é mostrado na Figura 3.5. Percebe-se que as curvas são muito semelhantes as da Figura 3.2, logo as mesmas conclusão são feitas. (Fim do exemplo) 


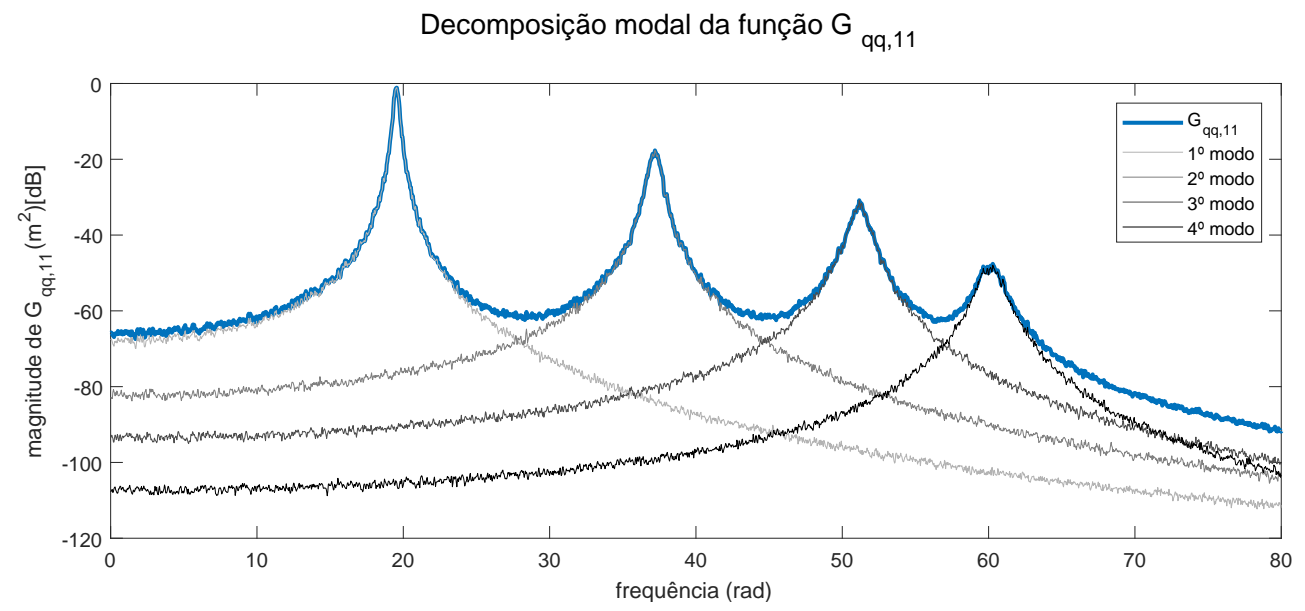

Figura 3.5: Exemplo da decomposição modal de uma PDS utilizando a hipótese de coordenadas modais não correlacionadas 


\section{4}

\section{Modelagens para a identificação de um sistema}

Modelar um sistema consiste em descrever seus respectivos fenômenos e respostas através de equações matemáticas. Na engenharia, deseja-se que estes modelos sejam o mais simples possível, mas que ainda assim consigam descrever com certa precisão o comportamento analisado. A procura pela simplicidade do modelo está relacionada com o desejo de desenvolver um processo de análise eficiente, sem que os erros nos resultados ultrapassem certa tolerância. Cabe ao analista responsável determinar o quão complexo e refinado o modelo deve ser.

A escolha de uma modelagem apropriada consiste na correta definição da família de modelos, e consequentemente na teoria associada a ela. Em análise modal, o processo de identificação é a principal etapa e tem seus respectivos métodos desenvolvidos em função do modelo do sistema. Por isso, uma boa compreensão dos modelos é essencial para o pleno entendimento desses métodos. Este capítulo inicia com a descrição das modelagens utilizadas pelos métodos de identificação mais populares e que serão apresentados nos capítulos 6 e 7 .

$\mathrm{Na}$ análise modal operacional, é preciso que os modelos apresentem processos estocásticos para contemplar a aleatoriedade do sistema. Assim, os forçamentos do ambiente (desconhecidos e não mensuráveis) e os ruídos dos sensores podem passar a ser incorporados no modelo. Não são descartados nos modelos os forçamentos determinísticos, uma vez que eles podem ser usados em conjunto com os forçamentos estocásticos para obter melhores resultados. Esse processo é conhecido na literatura como OMAX (Operational Modal Analysis with eXogenous inputs). Além disso, analogias entre os sistemas determinísticos e estocásticos podem ser realizadas. Essa comparação facilita a compreensão dos métodos de identificação, já que muitos foram estendidos da análise modal experimental (EMA).

O primeiro modelo analisado neste capítulo é o de espaço de estado e tem sua origem na teoria de controle. A modelagem apresentada neste capítulo se difere um pouco da já introduzida pela Eq. (2-77). Ela é utilizada em métodos de identificação de parâmetros como o ERA (Eigensystem Realization Algorithm) e o de subespaço estocástico. O segundo modelo analisado será o 
ARMA (Auto-Regression Moving Average).

\section{1}

\section{Modelo de espaço de estado}

Uma estrutura é sempre contínua e não linear. No entanto, muitas vezes, os modelos matemáticos utilizados para descrever o comportamento dessas estruturas contém simplificações tais como a discretização espacial e a linearização do sistema. Um exemplo é método de elementos finitos, que aproxima um sistema contínuo por outro similar com número finito de graus de liberdade (coordenadas). O modelo de um sistema linear unidirecional discretizado no espaço é representado, na maioria dos casos, por um sistema de equações diferenciais ordinárias de segunda ordem na forma

$$
\mathcal{M} \ddot{\mathbf{q}}(t)+\mathcal{C} \dot{\mathbf{q}}(t)+\mathcal{K} \mathbf{q}(t)=\mathcal{B} \mathbf{u}(t),
$$

onde $\mathcal{M}, \mathcal{C}$ e $\mathcal{K} \in \mathbb{R}^{n \times n}$ são novamente as matrizes de inércia, amortecimento e rigidez, respectivamente, e $n$ é o número de graus de liberdade do sistema linear. O vetor $\mathbf{q}(t) \in \mathbb{R}^{n}$ representa os deslocamentos dos respectivos graus de liberdade, e $\mathcal{B} \in \mathbb{R}^{n \times n_{i}}$ é a matriz de influência que determina a posição onde os forçamentos $\mathbf{u}(t) \in \mathbb{R}^{n_{i} \times 1}$ estão sendo aplicados. A constante $n_{i}$ representa o número dos forçamentos atuantes no modelo discretizado.

A equação (4-1) consiste em um sistema de equações diferenciais de segunda ordem e pode ser transformado em um de primeira ordem de diversas formas, sendo uma delas a já apresentada pela Eq. (2-77). Nas próximas seções desse capítulo, o sistema de primeira ordem será apresentado primeiro no domínio do tempo de forma contínua e depois de forma discretizada. Sua versão no domínio da frequência também será apresentada para que a chamada função de transferência possa ser parametrizada e os parâmetros modais extraídos.

\subsection{1}

\section{Modelo contínuo}

Utilizando a Eq. (4-1), um sistema pode ser modelado na forma de estado de espaço através de,

$$
\dot{\mathbf{x}}(t)=\mathbf{A}_{c} \mathbf{x}(t)+\mathbf{B}_{c} \mathbf{u}(t)
$$

onde,

$$
\mathbf{x}=\left[\begin{array}{c}
\mathbf{q} \\
\dot{\mathbf{q}}
\end{array}\right], \quad \mathbf{A}_{c}=\left[\begin{array}{cc}
0 & \mathbf{I} \\
-\mathcal{M}^{-1} \mathcal{K} & -\mathcal{M}^{-1} \mathcal{C}
\end{array}\right], \quad \mathbf{B}_{c}=\left[\begin{array}{c}
0 \\
\mathcal{M}^{-1} \mathcal{B}
\end{array}\right]
$$

O vetor $\mathbf{x}(t) \in \mathbb{R}^{m}$ é novamente chamado de vetor de estado, onde $m=2 n$ é a ordem do sistema de espaço de estado e é geralmente um importante 
parâmetro a ser observado durante a identificação de parâmetros. A matriz $\mathbf{A}_{c} \in \mathbb{R}^{m \times m}$ é chamada de matriz dinâmica do sistema e contém informações sobre os parâmetros modais (frequência natural, fator de amortecimento e modos) através de seus autovalores e autovetores. A matriz $\mathbf{B}_{c}$ é a matriz de entrada que representa a influência do forçamento determinístico nos estados do sistema. O subscrito $\square_{c}$ na Eq. (4-2) significa que o sistema é contínuo no tempo.

Quando realizada algumas observações no sistema, sensores são sempre utilizados para medir suas grandezas físicas como aceleração, deslocamento, deformação, etc. As observações podem ser definidas, na maioria dos casos, em função das variáveis de deslocamento, velocidade e aceleração. Dessa forma, pode-se montar a equação

$$
\mathbf{y}(t)=\mathbf{P}_{a} \ddot{\mathbf{q}}(t)+\mathbf{P}_{v} \dot{\mathbf{q}}(t)+\mathbf{P}_{d} \mathbf{q}(t),
$$

onde $\mathbf{y}(t) \in \mathbb{R}^{n_{o} \times 1}$ é o vetor de observações e $n_{o}$ é o número de observações (saídas ou respostas) medidas pelos sensores. As matrizes $\mathbf{P}_{a}, \mathbf{P}_{v}$ e $\mathbf{P}_{d} \in \mathbb{R}^{n_{o} \times n}$ são as matrizes de influência para a aceleração, velocidade e deslocamento. Ao explicitar $\ddot{\mathbf{q}}(t)$ na Eq. (4-1) e substituir o resultado na Eq. (4-3), obtêm-se

$$
\mathbf{y}(t)=\mathbf{P}_{a} \mathcal{M}^{-1}[\mathcal{B} \mathbf{u}(t)-\mathcal{C} \dot{\mathbf{q}}(t)-\mathcal{K} \mathbf{q}(t)]+\mathbf{P}_{v} \dot{\mathbf{q}}(t)+\mathbf{P}_{d} \mathbf{q}(t),
$$

onde percebe-se que as observações são expressas apenas em função dos estados $\mathbf{x}(t)$ (deslocamento e velocidade) e das entradas $\mathbf{u}(t)$. Dessa forma, pode-se reescrever a Eq. (4-4) de forma semelhante a Eq. (4-2) como sendo

$$
\mathbf{y}(t)=\mathbf{C x}(t)+\mathbf{D} \mathbf{u}(t)
$$

onde,

$$
\mathbf{C}=\left[\begin{array}{lll}
\mathbf{P}_{d}-\mathbf{P}_{a} \mathcal{M}^{-1} \mathcal{K} & \mathbf{P}_{v}-\mathbf{P}_{a} \mathcal{M}^{-1} \mathcal{C}
\end{array}\right], \quad \mathbf{D}=\mathbf{P}_{a} \mathcal{M}^{-1} \mathcal{B}
$$

O par composto pelas Eq. (4-2) e Eq. (4-5) representa a modelagem de espaço de estado no tempo contínuo. Mesmo que coerente com a natureza dos fenômenos físicos do sistema, essa modelagem contínua não sendo suficiente na prática, já que durante a aquisição e processamento do sinal existe sempre uma etapa de digitalização dos dados. Por este motivo, será apresentado a seguir uma versão discretizada no tempo para modelo de estado de espaço conforme mostrado em [37]. Para isso, é necessário que a hipótese de ordem zero entre amostras consecutivas seja imposta. Essa hipótese permite grande simplificação e consiste na ideia de que os valores de uma determinada variável se mantém constante entre as amostras consecutivas. Quando o intervalo de tempo entre essas amostras é relativamente pequeno, os erros inseridos por 
essa hipótese são desprezíveis.

\subsection{2}

\section{Modelo discreto}

Conhecendo as condições iniciais do sistema $\mathbf{x}\left(t_{0}\right)$ em um instante $t=t_{0}$ e o forçamento $\mathbf{u}(t)$, a solução geral para a equação diferencial ordinária (4-2) pode ser expressa, para $t>t_{0}$, como sendo a soma da solução homogênea com uma particular, resultando em

$$
\mathbf{x}(t)=e^{\mathbf{A}_{c}\left(t-t_{0}\right)} \mathbf{x}\left(t_{0}\right)+\int_{t_{0}}^{t} e^{\mathbf{A}_{c}(t-\tau)} \mathbf{B}_{c} \mathbf{u}(\tau) d \tau .
$$

Através dessa função, pode-se expressar a evolução do estado do sistema em função das condições iniciais e forçamento. Ao supor que os estados e forçamentos podem ser avaliados em intervalos de tempo $\Delta t$ igualmente espaçados, pode-se reescrever (4-6) na sua forma discreta. Substituindo $t=$ $(k+1) \Delta t$ e $t_{0}=k \Delta t$ em $(4-6)$, obtêm-se

$$
\mathbf{x}[(k+1) \Delta t]=e^{\mathbf{A}_{c} \Delta t} \mathbf{x}(k \Delta t)+\int_{k \Delta t}^{(k+1) \Delta t} e^{\mathbf{A}_{c}[(k+1) \Delta t-\tau]} \mathbf{B}_{c} \mathbf{u}(\tau) d \tau .
$$

Supondo a hipótese de ordem zero para $\mathbf{u}(\tau)$ e de que $\mathbf{B}_{c}$ é constante, a equação (4-7) pode ser reescrita utilizando uma mudança de variável $\tau^{\prime}=(k+1) \Delta t-\tau$, resultando em

$$
\mathbf{x}[(k+1) \Delta t]=e^{\mathbf{A}_{c} \Delta t} \mathbf{x}(k \Delta t)+\left[\int_{0}^{\Delta t} e^{\mathbf{A}_{c} \tau^{\prime}} d \tau^{\prime} \mathbf{B}_{c}\right] \mathbf{u}(k \Delta t) .
$$

Ao definir

$$
\begin{aligned}
\mathbf{A} & =e^{\mathbf{A}_{c} \Delta t} \\
\mathbf{B} & =\int_{0}^{\Delta t} e^{\mathbf{A}_{c} \tau^{\prime}} d \tau^{\prime} \mathbf{B}_{c} \\
\mathbf{x}(k+1) & =\mathbf{x}[(k+1) \Delta t] \\
\mathbf{u}(k) & =\mathbf{u}(k \Delta t)
\end{aligned}
$$

a equação (4-8) pode ser reescrita em uma forma compacta através de

$$
\mathbf{x}(k+1)=\mathbf{A} \mathbf{x}(k)+\mathbf{B u}(k) ; \quad k=0,1,2, \ldots
$$

Utilizando as mesmas hipóteses de discretização feitas acima, a equação (4-5) também pode ser reescrita na forma

$$
\mathbf{y}(k)=\mathbf{C x}(k)+\mathbf{D u}(k) .
$$

As equações (4-10) e (4-11) representam a modelagem de espaço de estado no tempo discreto. Através dela, é possível relacionar diretamente os 
sinais discretos da entrada com os de saída obtidos durante experimentos ou simulações. As relações entre as matrizes da modelagem contínua e discreta, definidas pela Eq. (4-9), são importantes durante o processo de identificação, já que os métodos identificam o sistema discreto e o que se deseja são os parâmetros do sistema contínuo.

As modelagens apresentadas até então não contemplam as possíveis excitações aleatórias e incertezas dos sensores, fenômenos comuns em análise modal. Por este motivo, modifica-se o modelo de estado de espaço discreto adicionando dois vetores, $\mathbf{w}(k) \in \mathbb{R}^{m}$ e $\mathbf{v}(k) \in \mathbb{R}^{n_{o}}$. As componentes desses vetores consistem em processos estocásticos classificados como ruídos brancos não correlacionados e de média zero. Assim, as Eqs. (4-10/4-11) se tornam

$$
\begin{gathered}
\mathbf{x}(k+1)=\mathbf{A} \mathbf{x}(k)+\mathbf{B u}(k)+\mathbf{w}(k) \\
\mathbf{y}(k)=\mathbf{C x}(k)+\mathbf{D u}(k)+\mathbf{v}(k) .
\end{gathered}
$$

O vetor $\mathbf{w}(k)$ representa os possíveis forçamentos aleatórios do ambiente (vento, ondas, movimentos sísmicos, excitações de base, etc), enquanto o vetor $\mathbf{v}(k)$ representa apenas os ruídos dos sensores. As matrizes de covariância entre esses processos são definidos como

$$
\mathbf{E}\left(\left[\begin{array}{l}
\mathbf{w}(p) \\
\mathbf{v}(p)
\end{array}\right]\left[\begin{array}{ll}
\mathbf{w}(g)^{T} & \mathbf{v}(g)^{T}
\end{array}\right]\right)=\left[\begin{array}{cc}
Q & S \\
S^{T} & R
\end{array}\right] \delta_{p g},
$$

onde $\mathbf{E}(\square)$ significa valor esperado e $\delta_{p g}$ o operador delta de Kronecker.

Como apenas sistemas lineares invariantes no tempo são tratados nesta dissertação, pode-se dividir os estados $\mathbf{x}(k)$ e as observações do sistema $\mathbf{y}(k)$ em relação as suas contribuições determinísticas e estocásticas,

$$
\mathbf{x}(k)=\mathbf{x}^{d}(k)+\mathbf{x}^{s}(k) \quad e \quad \mathbf{y}(k)=\mathbf{y}^{d}(k)+\mathbf{y}^{s}(k) .
$$

As Eqs. (4-12/4-13) podem então ser decompostas em dois subsistemas, sendo um deles determinístico

$$
\begin{gathered}
\mathbf{x}^{d}(k+1)=\mathbf{A} \mathbf{x}^{d}(k)+\mathbf{B u}(k) \\
\mathbf{y}^{d}(k)=\mathbf{C} \mathbf{x}^{d}(k)+\mathbf{D u}(k),
\end{gathered}
$$

e outro estocástico,

$$
\begin{gathered}
\mathbf{x}^{s}(k+1)=\mathbf{A} \mathbf{x}^{s}(k)+\mathbf{w}(k) \\
\mathbf{y}^{s}(k)=\mathbf{C} \mathbf{x}^{s}(k)+\mathbf{v}(k) .
\end{gathered}
$$

A comparação feita a seguir entre esses dois subsistemas facilitará a compreensão de como os métodos de identificação dos sistemas determinísticos 
(desenvolvidos para análise modal experimental) podem ser estendidos aos métodos de identificação dos sistemas estocásticos (análise modal operacional). No domínio do tempo, os métodos de identificação de sistemas determinísticos utilizam as funções de resposta ao impulso unitário, enquanto para os sistemas estocásticos as funções de correlação dos sinais de saída são utilizadas. A justificativa para a utilização dessas funções é a possibilidade de decompôlas em relação aos parâmetros modais, como mostrado nos capítulos 2 e 3 . A seguir, ambas as funções são representadas de forma similar, através dos chamados parâmetros de Markov. [37][38][39].

\subsection{3}

\section{Parâmetros de Markov - Sistemas determinísticos}

Para o sistema determinístico, é possível relacionar os sinais de entrada e saída através das funções de resposta ao pulso unitário. Quando estritas no modelo de estado de espaço, essas funções podem ser expressas como uma sequência ponderada dos sinais de entrada, onde os pesos são os chamados parâmetros de Markov.

A resposta de um sistema com condições iniciais nulas pode ser obtida através das Eq. (4-15/4-16), resultando em

$$
\begin{aligned}
\mathbf{x}^{d}(0) & =0 \\
\mathbf{y}^{d}(0) & =\mathbf{D u}(0) \\
\mathbf{x}^{d}(1) & =\mathbf{B u}(0) \\
\mathbf{y}^{d}(1) & =\mathbf{C B u}(0)+\mathbf{D u}(1) \\
\mathbf{x}^{d}(2) & =\mathbf{A B u}(0)+\mathbf{B u}(1) \\
\mathbf{y}^{d}(2) & =\mathbf{C A B u}(0)+\mathbf{C B u}(1)+\mathbf{D}(2) \\
\vdots & \\
\mathbf{x}^{d}(k) & =\sum_{i=1}^{k} \mathbf{A}^{i-1} \mathbf{B u}(k-i) \\
\mathbf{y}^{d}(k) & =\sum_{i=1}^{k} \mathbf{C A}^{i-1} \mathbf{B u}(k-i)+\mathbf{D u}(k)
\end{aligned}
$$

Se para cada entrada for realizado um pulso inicial de forma independentes, isso é, $u_{i}(0)=1\left(i=1,2, \ldots, n_{i}\right)$ e $u_{i}(k)=0(k=1,2, \ldots)$, a resposta da Eq. (4-20) pode ser escrita como 


$$
\mathbf{y}^{d}(k)=\sum_{i=0}^{k} \mathbf{Y}(i) \mathbf{u}(k-i)
$$

onde

$$
\mathbf{Y}(0)=\mathbf{D}, \mathbf{Y}(1)=\mathbf{C B}, \mathbf{Y}(2)=\mathbf{C A B}, \ldots, \mathbf{Y}(i)=\mathbf{C A}^{i-1} \mathbf{B}
$$

são matrizes constantes chamadas de parâmetros de Markov. Essas matrizes representam a resposta ao pulso unitário do sistema, e por isso devem ser únicas.

\subsection{4}

\section{Parâmetros de Markov - Sistemas estocásticos}

Em relação ao sistema estocástico, será mostrado a seguir que as matrizes de correlação dos sinais de saída possuem a mesma estrutura e informação que os parâmetros de Markov definidos anteriormente. Para tal demonstração, algumas definições precisam ser feitas.

Supõe-se primeiramente que as componentes do vetor de estado estocástico, $\mathbf{x}^{s}(k)$, são processos estacionários e com média zero, resultando em

$$
\begin{aligned}
\mathbf{E}\left[\mathbf{x}^{s}(k)\right] & =0 \\
\mathbf{E}\left[\mathbf{x}^{s}(k) \mathbf{x}^{s^{T}}(k)\right] & \triangleq \mathbf{R}_{x x}^{s},
\end{aligned}
$$

onde a matriz de correlação dos estados $\mathbf{R}_{x x}^{s}$ é constante (independente do tempo). Além disso, supõe-se também que os vetores $\mathbf{w}(k)$ e $\mathbf{v}(k)$ não são correlacionados aos estados $\mathbf{x}^{s}(k)$ em um mesmo instante de tempo. Isso é representado pela equação

$$
\begin{aligned}
& \mathbf{E}\left[\mathbf{x}^{s}(k) \mathbf{w}^{T}(k)\right]=0, \\
& \mathbf{E}\left[\mathbf{x}^{s}(k) \mathbf{v}^{T}(k)\right]=0 .
\end{aligned}
$$

Utilizando essas duas hipóteses, algumas matrizes de correlação são definidas como feito em [38]. Inicia-se com a matriz de correlação dos vetores de estado em um mesmo instante, dada pela equação de Lyapunov

$$
\begin{aligned}
\mathbf{R}_{x x}^{s} & =\mathbf{E}\left[\mathbf{x}^{s}(k+1)\left[\mathbf{x}^{s^{T}}(k+1)\right]\right] \\
& =\mathbf{E}\left[\left[\mathbf{A} \mathbf{x}^{s}(k)+\mathbf{w}(k)\right] \cdot\left[\mathbf{A} \mathbf{x}^{s}(k)+\mathbf{w}(k)\right]^{T}\right] \\
& =\mathbf{A E}\left[\mathbf{x}^{s}(k) \mathbf{x}^{s^{T}}(k)\right] \mathbf{A}^{T}+\mathbf{E}\left[\mathbf{w}^{T}(k) \mathbf{w}(k)\right] \\
& =\mathbf{A} \mathbf{R}_{x x}^{s} \mathbf{A}^{T}+\mathbf{Q}
\end{aligned}
$$

As matrizes de correlação entre os vetores de estado no instante $k+1$ com os 
vetores de saída no instante $k$ são definidos como sendo

$$
\begin{aligned}
\mathbf{G} & \triangleq \mathbf{E}\left[\mathbf{x}^{s}(k+1) \mathbf{y}^{s^{T}}(k)\right] \\
& =\mathbf{E}\left[\left[\mathbf{A} \mathbf{x}^{s}(k+1)+\mathbf{w}(k)\right] \cdot\left[\mathbf{C} \mathbf{x}^{s}(k)+\mathbf{v}(k)\right]^{T}\right] \\
& =\mathbf{A} \mathbf{E}\left[\mathbf{x}^{s}(k) \mathbf{x}^{s^{T}}(k)\right] \mathbf{C}^{T}+\mathbf{E}\left[\mathbf{w}(k) \mathbf{v}^{T}(k)\right] \\
& =\mathbf{A} \mathbf{R}_{x x}^{s} \mathbf{C}^{T}+\mathbf{S}
\end{aligned}
$$

Já as matrizes de correlação entre os vetores de saída são definidos como

$$
\mathbf{R}_{y y}^{s}\left(\tau_{k}\right) \triangleq \mathbf{E}\left[\mathbf{y}^{s}\left(k+\tau_{k}\right) \mathbf{y}^{s^{T}}(k)\right]
$$

onde $\tau_{k}$ é um número inteiro que representa a defasagem discreta entre os sinais no cálculo da correlação. Para $\tau_{k}=0$, têm-se

$$
\begin{aligned}
\mathbf{R}_{y y}^{s}(0) & =\mathbf{E}\left[\mathbf{y}^{s}(k) \mathbf{y}^{s^{T}}(k)\right] \\
& =\mathbf{E}\left[\left[\mathbf{C} \mathbf{x}^{s}(k)+\mathbf{v}(k)\right] \cdot\left[\mathbf{C} \mathbf{x}^{s}(k)+\mathbf{v}(k)\right]^{T}\right] \\
& =\mathbf{C E}\left[\mathbf{x}^{s}(k) \mathbf{x}^{s^{T}}(k)\right] \mathbf{C}^{T}+\mathbf{E}\left[\mathbf{v}(k) \mathbf{v}^{T}(k)\right] \\
& =\mathbf{C R}_{x x}^{s} \mathbf{C}^{T}+R .
\end{aligned}
$$

Já para $\tau_{k}>0$, utilizando a Eq. (4-25), obtêm-se

$$
\mathbf{R}_{y y}^{s}\left(\tau_{k}\right)=\mathbf{C A}^{\tau_{k}-1} \mathbf{G}
$$

Ao comparar a Eq. (4-22) com a Eq. (4-28), percebe-se que ambas possuem a mesma estrutura. Além disso ambas contêm as mesmas informações modais já que estes parâmetros estão contidos em A como será mostrado mais adiante. Este fato demonstra que, para os métodos de identificação de sistemas no domínio do tempo que utilizam a modelagem de espaço de estado, pode-se substituir as funções de resposta ao impulso pelas funções de correlação dos sinais de saída quando realizado uma análise modal operacional. Essa mesma conclusão foi obtida através das decomposições modais feitas nos capítulos 2 e 3. A Tabela 4.1 apresenta uma comparação mais direta entre os dois modelos de estado de espaço.

\subsection{5}

\section{Domínio da frequência}

Para extrair os parâmetros modais a partir da modelagem de espaço de estado, a transformada- $z$ das Eqs. (4-17/4-18) são necessárias. O objetivo é obter uma expressão para a função de transferência e em seguida calcular os polos do sistema. Ao realizar a transformada em ambos os lados das Eqs. 
Tabela 4.1: Comparação entre sistemas determinísticos e estocásticos

\begin{tabular}{|c|c|}
\hline Sistema determinístico & Sistema estocásticos \\
\hline $\mathbf{A}, \mathbf{C}, \mathbf{B}$ e D & $\mathbf{A}, \mathbf{C}, \mathbf{G}$ e $\boldsymbol{\Lambda}_{0}$ \\
$\mathbf{Y}(0)=D$ & $\mathbf{R}_{y y}^{s}(0)=\mathbf{C R}_{x x}^{s} \mathbf{C}^{T}+R$ \\
$\mathbf{Y}(0)=\mathbf{C A}^{i-1} \mathbf{B}$ & $\mathbf{R}_{y y}^{s}\left(\tau_{k}\right)=\mathbf{C A}^{\tau_{k}-1} \mathbf{G}$ \\
\hline
\end{tabular}

(4-17/4-18), obtêm-se

$$
\begin{aligned}
z \tilde{\mathbf{x}}(z) & =\mathbf{A} \tilde{\mathbf{x}}(z)+\tilde{\mathbf{w}}(z) \\
\tilde{\mathbf{y}}(z) & =\mathbf{C} \tilde{\mathbf{x}}(z)+\tilde{\mathbf{v}}(z)
\end{aligned}
$$

Explicitando $\tilde{\mathbf{x}}(z)$ na Eq. (4-29) e substituindo o resultado na Eq. (4-30), obtêm-se

$$
\begin{aligned}
\tilde{\mathbf{y}}(z) & =\mathbf{C}(z \mathbf{I}-\mathbf{A})^{-1} \tilde{\mathbf{w}}(z)+\tilde{\mathbf{v}}(z) \\
& =\tilde{\mathbf{H}}(z) \tilde{\mathbf{w}}(z)+\tilde{\mathbf{v}}(z),
\end{aligned}
$$

onde I é a matriz identidade e $\tilde{\mathbf{H}}(z)$ a função de transferência. Pela regra de Cramer, observa-se que

$$
\tilde{\mathbf{H}}(z)=\mathbf{C} \frac{\operatorname{adj}(z \mathbf{I}-\mathbf{A})}{\operatorname{det}(z \mathbf{I}-\mathbf{A})},
$$

onde $\operatorname{det}(\square)$ representa o determinante e $\operatorname{adj}(\square)$ a matriz adjunta. O denominador da função de transferência é então definido por $\operatorname{det}(z \mathbf{I}-\mathbf{A})$, que ao ser igualado a zero corresponde ao polinômio característico de A. Dessa forma, os polos da função de transferência são obtido realizando o cálculo dos autovalores de $\mathbf{A}$.

Ao definir $\lambda_{i}$ e $\phi_{i}$ como os autovalores e autovetores de $\mathbf{A}$, os parâmetros modais do sistema podem ser extraídos através de

$$
\mu_{i}=\frac{\log \left(\lambda_{i}\right)}{\Delta t}, \quad f_{i}=\frac{\left|\mu_{i}\right|}{2 \pi}, \quad \zeta_{i}=\frac{-\operatorname{Re}\left(\mu_{i}\right)}{\left|\mu_{i}\right|}, \quad \psi_{i}=\mathbf{C} \phi_{i} ; \quad i=1, \ldots, n
$$

onde $\mu_{i}$ são os polos do sistema no modelo contínuo, $f_{i}$ as frequências naturais, $\zeta_{i}$ os fatores de amortecimento e $\psi_{i}$ os modos. Devido ao modelo de estado de espaço, os $m$ autovalores aparecem em pares de complexos conjugados. Como $m=2 n$, apenas $n$ (números de graus de liberdade) parâmetros modais podem ser obtidos. 


\section{2}

\section{Modelo ARMA}

Um outro tipo de modelagem muito utilizada tanto em processamento de sinais quanto em análise modal é chamada de ARMA [14][27][31]. Essa modelagem consiste em descrever a resposta de um sistema a partir das equações de diferenças

$$
\mathbf{y}(k)=\sum_{r=1}^{N} \mathbf{A}_{r} \mathbf{y}(k-r)+\sum_{r=0}^{M} \mathbf{B}_{r} \mathbf{u}(k-r),
$$

onde $\mathbf{u}(k)$ e $\mathbf{y}(k)$ são os sinais discretos da entrada e de resposta, respectivamente. As matrizes $\mathbf{A}_{r} \in \mathbb{R}^{n_{o} \times n_{o}}$ correspondem à parte $\mathrm{AR}$ (autoregressive) do modelo, uma vez que a resposta em um determinado instante é modelado como uma regressão das respostas em instantes anteriores. Estas matrizes contêm as informações modais do sistema e são usadas como fonte para a identificação dos parâmetros. Já as matrizes $\mathbf{B}_{r} \in \mathbb{R}^{n_{o} \times n_{i}}$ correspondem à parte MA (moving average) do modelo e representam a parte estatística e do forçamento que atua no sistema. Os parâmetros $N$ e $M$ neste caso correspondem ao número de matrizes necessárias para a boa representação do sistema.

Ao realizar a transformada- $z$ da Eq. (4-33), obtêm-se como resultado

$$
\tilde{\mathbf{y}}(z)=\sum_{r=1}^{N} \mathbf{A}_{r} z^{-r} \tilde{\mathbf{y}}(z)+\sum_{r=0}^{M} \mathbf{B}_{r} z^{-r} \tilde{\mathbf{u}}(z),
$$

onde na equação acima, a propriedade de translação no tempo da transformada-z foi utilizada. Rearrumando a equação como uma razão entre as transformadas dos sinais de resposta com as dos sinais de entrada, tem-se a função de transferência do sistema na forma

$$
\tilde{\mathbf{H}}(z)=\frac{\tilde{\mathbf{y}}(z)}{\tilde{\mathbf{u}}(z)}=\frac{\sum_{r=0}^{M} \mathbf{B}_{r}^{-r}}{1-\sum_{r=1}^{N} \mathbf{A}_{r} z^{-r}} .
$$

Através da Eq. (4-36), nota-se que apenas as matrizes $\mathbf{A}_{r}$ compõem o denominador e consequentemente somente elas interferem nos valores dos polos do sistema.

A extração dos parâmetros modais no modelo ARMA pode ser demonstrada através da resposta livre do sistema. Omitindo todos os sinais de entrada (forçamento), a Eq. (4-33) se reduz a

$$
\mathbf{y}(k)-\mathbf{A}_{1} \mathbf{y}(k-1)+\cdots+\mathbf{A}_{N} \mathbf{y}(k-N)=0 .
$$

Agrupando as últimas $\mathrm{N}$ respostas do sistema em um único vetor, obtêm-se 


$$
\mathbf{y}_{d}(k)=\left[\begin{array}{c}
\mathbf{y}(k-N+1) \\
\vdots \\
\mathbf{y}(k-1) \\
\mathbf{y}(k)
\end{array}\right] .
$$

Conhecido $\mathbf{y}(k)$, a resposta do sistema no passo seguinte, $\mathbf{y}(k+1)$, pode ser obtida através de uma matriz $\mathbf{A}_{d}$, chamada de matriz de acompanhamento, cuja forma é dada por

$$
\mathbf{A}_{d}=\left[\begin{array}{ccccc}
0 & \mathbf{I} & 0 & \ldots & 0 \\
0 & 0 & \mathbf{I} & \ldots & 0 \\
\vdots & \vdots & \vdots & \ddots & \vdots \\
0 & 0 & 0 & \ldots & \mathbf{I} \\
\mathbf{A}_{N} & \mathbf{A}_{N-1} & \mathbf{A}_{N-2} & \ldots & \mathbf{A}_{1}
\end{array}\right]
$$

I neste caso é a matriz identidade com dimensões iguais as das matrizes $\mathbf{A}_{r}$. Utilizando essa matriz de acompanhamento, pode-se transformar o vetor de resposta $\mathbf{y}_{d}$ de um instante $k$ para o próximo instante $k+1$. Isso é feito através da equação

$$
\mathbf{y}_{d}(k+1)=\mathbf{A}_{d} \mathbf{y}_{d}(k) .
$$

A relação encontrada na Eq. (4-40) é utilizada a seguir para mostrar como os parâmetros modais podem ser extraídos através de autovalores e autovetores da matriz $\mathbf{A}_{d}$. Para isso, a hipótese de que a resposta de um sistema pode ser escrita como uma superposição de modos é utilizada. Se apenas o $i$-ésimo modo for excitado, tem-se a resposta do sistema definida como

$$
\mathbf{y}(k)=\boldsymbol{\psi}_{i} \lambda_{i}^{k},
$$

onde $\boldsymbol{\psi}_{i}$ é o $i$-ésimo modo do sistema e $\lambda_{i}=e^{\mu_{i} \Delta t}$ o polo do sistema discreto associado ao $i$-ésimo polo contínuo $\mu_{i}$. Substituindo a Eq. (4-41) na Eq. (4-38) obtêm-se

$$
\mathbf{y}_{d, i}(k)=\left[\begin{array}{c}
\boldsymbol{\psi}_{i} \mu_{i}^{-N+1} \\
\vdots \\
\boldsymbol{\psi}_{i} \mu_{i}^{-1} \\
\boldsymbol{\psi}_{i}
\end{array}\right] \mu_{i}^{k}=\boldsymbol{\psi}_{d, i} \mu_{i}^{k}
$$

Ao substituir o resultado acima na Eq. (4-40), o seguinte problema de autovalor é obtido:

$$
\mu_{i} \boldsymbol{\psi}_{i, d}=\mathbf{A}_{d} \boldsymbol{\psi}_{i, d}
$$

A equação (4-43) relaciona os parâmetros modais do sistema com os autovalores e autovetores da matriz de acompanhamento $\mathbf{A}_{d}$. O formato do i-ésimo modo é obtidos como as últimas $n_{o}$ linhas do autovetor $\boldsymbol{\psi}_{i, d}$. 


\section{5}

\section{Influência do ruído nas funções de correlação}

Os métodos de identificação no domínio do tempo, como por exemplo o método de subespaço estocástico, ERA, ARMA e de decomposições ortogonais, utilizam as funções de correlação como fonte de informação. Durante os experimentos, os sinais observados possuem sempre uma certa quantidade ruídos devido aos sensores e a eletrônica envolvida. Neste capítulo realizase um estudo sobre a influência que esses ruídos exercem nas estimativas das funções de correlações. Dependendo do tipo resposta medida (deslocamento, velocidade ou aceleração), mostra-se que o ruído do sensor deforma as funções de correlação de diferente formas. Isso ocorre pela necessidade de passar os sinais por filtros de integração ou derivação.

Esse capítulo inicia definindo a modelagem para este tipo de ruído. Em seguida mostra-se como esse ruído deforma as funções de correlação, tenha o sinal passado ou não por filtros de integração ou derivação. Durante esta análise, uma importante propriedade das derivadas das funções de correlações é apresentada. Devido a inevitável digitalização dos sinais durante a aquisição de dados em um experimento, o estudo é realizado com sinais discretos no tempo. Nesta dissertação, as integrações e derivações são realizadas por diferentes filtros, cujas demonstrações estão fora do escopo deste trabalho e podem ser encontradas em [26][27][40][41][42].

\section{1}

\section{Influência dos ruídos nas funções de correlação}

Os deslocamentos dos graus de liberdade de um sistema são novamente representados aqui pelo vetor deslocamento $\mathbf{q}(k) \in \mathbb{R}^{n}$. Os possíveis ruídos dos sensores encontrados nas observações do sistema são representados pelo vetor $\mathbf{v}(k) \in \mathbb{R}^{n}$, que ao ser somado à resposta do sistema, resulta em

$$
\mathbf{y}(k)=\mathbf{q}(k)+\mathbf{v}(k) .
$$

O vetor $\mathbf{y}(k) \in \mathbb{R}^{n}$ representa o sinal discreto da resposta do sistema quando observada por sensores de deslocamento. Por simplicidade supõem-se que todos os graus de liberdade foram observados $\left(n_{o}=n\right)$. Quando os sensores utilizados medem velocidade ou aceleração, a Eq. (5-1) se transforma em 


$$
\dot{\mathbf{y}}(k)=\dot{\mathbf{q}}(k)+\mathbf{v}(k)
$$

$\mathrm{Ou}$

$$
\ddot{\mathbf{y}}(k)=\ddot{\mathbf{q}}(k)+\mathbf{v}(k) .
$$

Observa-se pelas Eqs. (5-2/5-3) que a modelagem do ruído no sinal é o mesmo, independente do tipo de resposta medida. Este ruído é geralmente modelado como um ruído branco, de média zero e não-correlacionado com os sinais de resposta do sistema. Uma importante característica do ruído branco é o fato de ser um processo ergódico. Pela definição, as estatísticas das variáveis aleatórias deste processo em cada instante de tempo são ditas iguais as estatísticas de uma realização do mesmo processo ao longo do tempo.

Pelas propriedades de um ruído branco discutidas na seção 3.3.1, o cálculo da matrizes de correlação do vetor $\mathbf{v}(k)$ é dado por

$$
\mathbf{R}_{v v}\left(\tau_{k}\right)=\mathbf{E}\left[\mathbf{v}(k) \mathbf{v}^{T}\left(k+\tau_{k}\right)\right]=\mathbf{Q}_{v} \delta\left(\tau_{k}\right),
$$

onde $\mathbf{Q}_{v} \in \mathbb{R}^{n \times n}$ é a matriz de covariância dos ruídos e $\delta\left(\tau_{k}\right)$ é a função impulso unitário discreto. Quando os ruídos não são correlacionados entre si, a matriz $\mathbf{Q}_{v}$ se torna diagonal.

A modelagem de um ruído é independente do tipo de resposta medida, como definido pelas Eqs. (5-1/5-2/5-3). Quando os sinais são medidos por diferentes tipos de sensores e convertidos para a mesma grandeza (deslocamento, velocidade ou aceleração), as características dos ruídos também são modificadas e consequentemente suas funções de correlação. Isso ocorre devido ao processo de integração ou derivação e pode apresentar diferentes resultados dependendo do filtro escolhido. Quando a correlação do deslocamento de um sistema é calculada a partir de um sinal medido por sensores de deslocamento, a distorção causada pelo ruído será diferente do caso onde o sinal é medido por sensores de aceleração e integrado duas vezes.

A seguir, serão analisados três casos onde as funções de correlação da velocidade de um sistema são calculadas, conforme mostra a Figura 5.1. Para o primeiro caso, o sinal da velocidade do sistema é obtido diretamente através de uma simulação. Em seguida o ruído é adicionado ao sinal e a função de correlação é estimada. Já no segundo caso, o sinal do deslocamento do sistema é obtido através de uma simulação. Em seguida o ruído é adicionado para só depois o sinal ser processado por um filtro de derivação. Após o sinal da velocidade ter sido obtido, a função de correlação é estimada. No terceiro e último caso, a aceleração do sistema é obtida através de uma simulação e o ruído é então adicionado. O sinal resultante é processado por um filtro de integração para só depois a função de correlação da velocidade ser estimada. Os três exemplos estudados ilustram como o ruído pode distorcer as funções 
de correlação após um processamento de integração ou derivação.

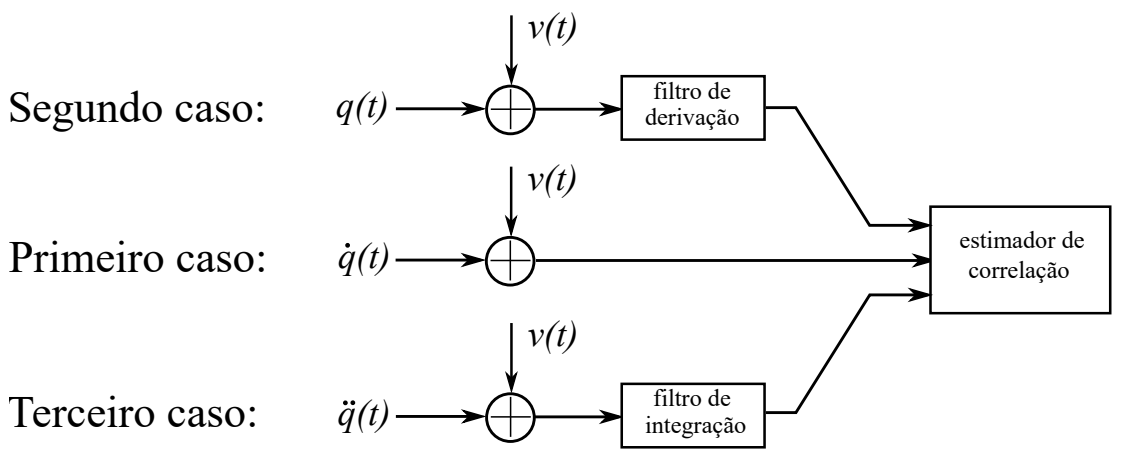

Figura 5.1: Casos analisados

Para exemplificar os resultados obtidos, realiza-se simulações numéricas para um sistema massa-mola-amortecedor de um grau de liberdade. A adição dos ruídos é realizado após as respectivas respostas terem sido ser obtidas. O ruído é modelado como um ruído branco, de média zero e distribuição de probabilidade Gaussiana. Seu desvio padrão é proporcional ao desvio padrão da resposta do sistema, variando entre $0 \%$ a $50 \%$ conforme mostra a Figura 5.2. Nessa seção, todas as funções de correlação são estimadas utilizando o método de Welch.

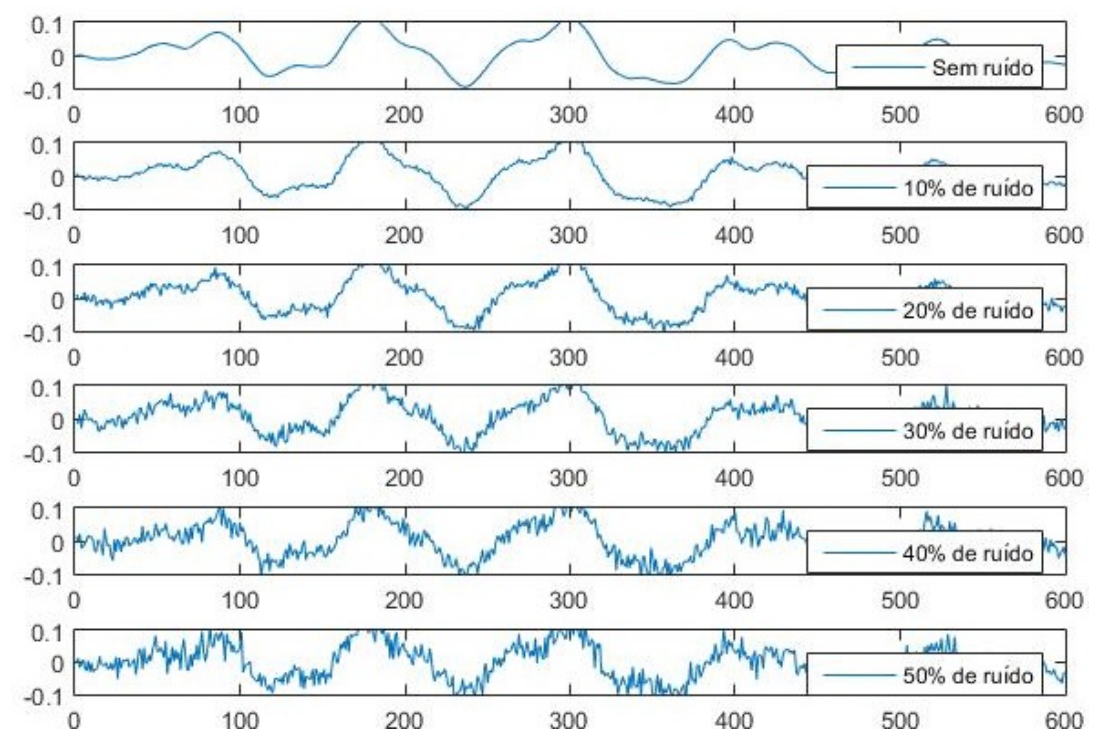

Figura 5.2: Respostas do sistema com adição de ruído

\subsection{1}

\section{Correlação da resposta do sistema com ruído}

Imagina-se que a velocidade de um sistema seja adquirida diretamente pelos sensores e que o ruído presente no sinal seja o ruído branco descrito acima. O sinal resultante consiste na soma de um sinal estacionário, mais um 
processo ergódico, como mostrado pela Eq. (4-2). A correlação desse sinal é calculada através da equação

$$
\begin{aligned}
\mathbf{R}_{\dot{y} \dot{y}}\left(\tau_{k}\right)= & \mathbf{E}\left[\dot{\mathbf{y}}(k) \dot{\mathbf{y}}^{T}\left(k+\tau_{k}\right)\right] \\
= & \mathbf{E}\left[[\dot{\mathbf{q}}(k)+\mathbf{v}(k)]\left[\dot{\mathbf{q}}^{T}\left(k+\tau_{k}\right)+\mathbf{v}^{T}\left(k+\tau_{k}\right)\right]\right] \\
= & \mathbf{E}\left[\dot{\mathbf{q}}(k) \dot{\mathbf{q}}^{T}\left(k+\tau_{k}\right)\right]+\mathbf{E}\left[\dot{\mathbf{q}}(k) \mathbf{v}^{T}\left(k+\tau_{k}\right)\right]+ \\
& +\mathbf{E}\left[\mathbf{v}(k) \dot{\mathbf{q}}^{T}\left(k+\tau_{k}\right)\right]+\mathbf{E}\left[\mathbf{v}(k) \mathbf{v}^{T}\left(k+\tau_{k}\right)\right] .
\end{aligned}
$$

Utilizando a hipótese de que o os sinais do ruído e as respostas do sistema não serem correlacionados, a Eq. (5-5) se reduz a

$$
\begin{aligned}
\mathbf{R}_{\dot{y} \dot{y}}\left(\tau_{k}\right) & =\mathbf{E}\left[\dot{\mathbf{q}}(k) \dot{\mathbf{q}}^{T}\left(k+\tau_{k}\right)\right]+\mathbf{E}\left[\mathbf{v}(k) \mathbf{v}^{T}\left(k+\tau_{k}\right)\right] \\
& =\mathbf{R}_{\dot{q} \dot{q}}\left(\tau_{k}\right)+\mathbf{R}_{v v}\left(\tau_{k}\right)
\end{aligned}
$$

As componentes $\mathbf{E}\left[\dot{\mathbf{q}}(k) \mathbf{v}^{T}\left(k+\tau_{k}\right)\right]$ e $\mathbf{E}\left[\mathbf{v}(k) \dot{\mathbf{q}}^{T}\left(k+\tau_{k}\right)\right]$ só se tornam zero quando uma quantidade suficiente de dados é utilizada. Esse número de amostras é proporcional ao desvio padrão do ruído, isto é, quanto maior for sua presença, mais amostras devem ser utilizadas para estimar as correlações.

A Eq. (5-6) monstra que as correlações dos sinais observados (medidos) correspondem a soma das correlações da velocidade com as correlações dos ruídos. Assim, a distorção causado pelo ruído é a sua própria matriz de correlações, que, através da Eq. (5-4), corresponde a um impulso unitário com magnitude igual a sua matriz de covariância. Isso significa que apenas em $\tau_{k}=0$ a correlação é permanentemente distorcida, independente do número de amostras utilizadas.

\section{Exemplo 5.1}

Para mostrar o resultado acima, simulou-se a resposta de um sistema massamola-amortecedor com $m=10 \mathrm{~kg}, c=25 \mathrm{~kg} / \mathrm{s}$ e $\mathrm{k}=40 \mathrm{kN} / \mathrm{m}$ e forçamento igual a uma realização de um ruído branco com média zero, desvio padrão de $100 N$ e distribuição de probabilidade Gaussiana. Após o cálculo da velocidade do sistema, sua correlação sem ruído foi estimada e é mostrada pela Figura 5.3a. Uma vez conhecido o sinal de velocidade, calculou-se o seu desvio padrão e em seguida gerou-se uma realização de um ruído branco com desvio padrão igual a 30\% do desvio padrão da velocidade. A função de correlação deste ruído foi estimada e é mostrado pela Figura 5.3b. Percebe-se que, como esperado, a função tem a forma de um impulso unitário e com magnitude igual a $9 \%$ do valor máximo da correlação da velocidade. Isso ocorre, porque no valor máximo $\left(\tau_{k}=0\right)$ o valor da correlação é igual a variância do sinal. Como o 
desvio padrão do ruído é $30 \%$ do desvio padrão da velocidade, a relação entre as variâncias é de $9 \%\left(0.3^{2}\right)$. Quando o sinal do ruido é somado ao da velocidade, tem-se um típico sinal experimental. A correlação deste sinal é mostrada na Figura 5.3c e mostra ser exatamente o resultado obtido pela Eq. (5-6). Percebese então que, em um sinal experimental, a função de correlação é afetada pelo ruído de forma permanente apenas em $\tau_{k}=0$.
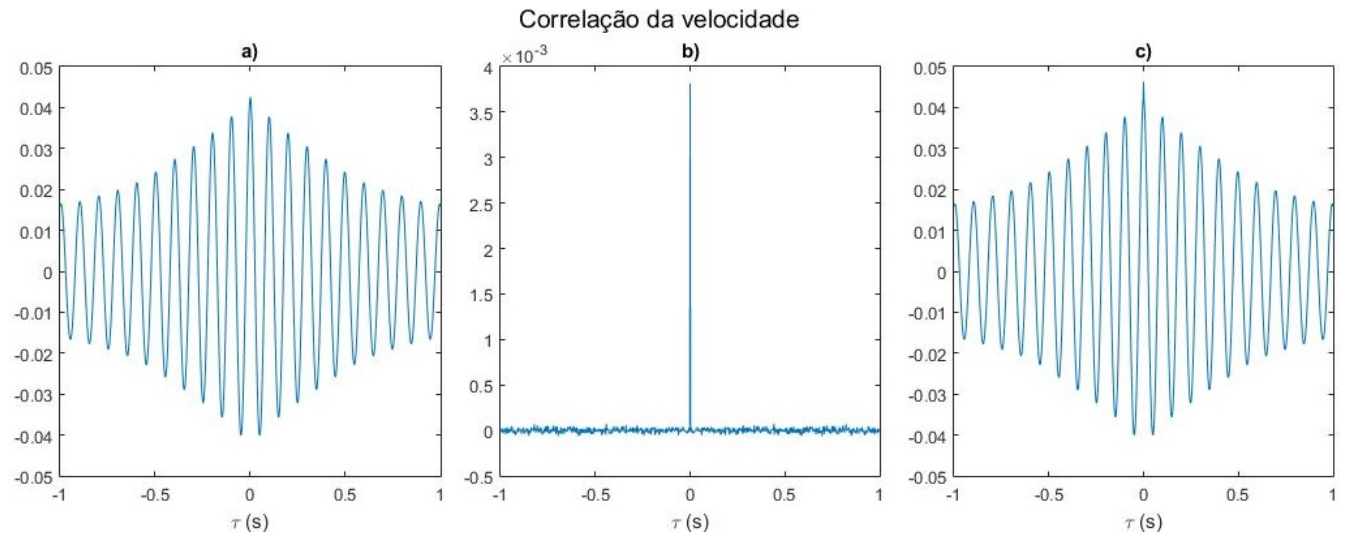

Figura 5.3: Correlações dos sinais: a) da resposta do sistema sem ruído, b) do ruído e c) da resposta do sistema com ruído

Para melhor demonstrar essa distorção, gerou-se sinais de ruído com diferentes valores para a porcentagem do seu desvio padrão. O resultado é mostrado na Figura 5.4. Percebe-se que a magnitude da distorção é proporcional ao quadrado da porcentagem do desvio padrão (variância). (Fim do exemplo)

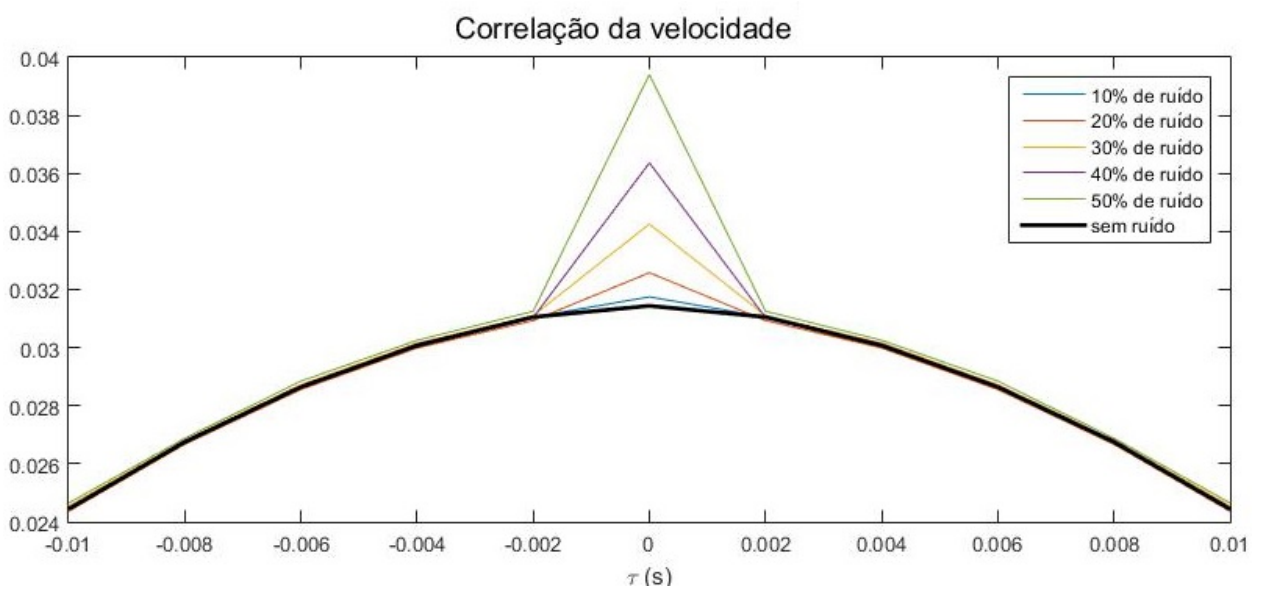

Figura 5.4: Distorção causada pelo ruído nas funções de correlação para diferentes proporções de ruído 


\section{1 .2}

\section{Correlação da derivada da resposta do sistema com ruído}

A seguir, repete-se a análise da seção anterior, no entanto partindo agora do sinal de deslocamento. Para obter a correlação da velocidade, é preciso então que o sinal medido seja processado por um filtro de derivação. Deseja-se analisar como a derivação afeta o ruído do sinal e consequentemente sua função de correlação.

Os sinais de deslocamento mais os de ruído são definidos pela Eq. (5-1), e repetido aqui por conveniência,

$$
\mathbf{y}(k)=\mathbf{x}(k)+\mathbf{v}(k) .
$$

Sendo a derivação um operador linear, ao aplica-lo na Eq. (5-7) obtêm-se

$$
\dot{\mathbf{y}}(k)=\dot{\mathbf{x}}(k)+\dot{\mathbf{v}}(k) .
$$

Nota-se que a Eq. (5-8) se diferencia da Eq. (5-2) pelo fato do ruído também ter passado pelo processo de derivação, não podendo mais ser classificado como um ruído branco. No domínio da frequência, a operação de derivação corresponde a multiplicar o respectivo espectro do sinal por $i \omega$, onde $i=\sqrt{-1}$ e $\omega$ é o valor da frequência. Dessa forma, o espectro do ruído branco não é mais constante após a derivação, e sim uma reta crescente. Por isso, pode-se afirmar que a derivação potencializa as frequências mais altas e suaviza as mais baixas.

A seguir utiliza-se uma simples aproximação para a derivada. Essa aproximação ajuda a compreender os efeitos causados pela derivação. Esse simples filtro possuí a forma

$$
\dot{\mathbf{s}}(k)=[\mathbf{s}(k)-\mathbf{s}(k-1)] f_{s},
$$

onde $\mathbf{s}(k)$ é um processo estacionário genérico e $f_{s}$ a frequência de amostragem. Para compreender os efeitos da derivação neste tipo de processo, calcula-se a seguir a média e variância da derivada do processo em função da média e variância do processo original. Para isso, observa-se pela Eq. (5-9) que a derivada é aproximada por uma soma de dois vetores aleatórias. Sendo eles parte e um processos estacionário, suas estatísticas são independente do instante de tempo. Assim, os valores esperados das derivadas são calculados por

$$
\begin{aligned}
\boldsymbol{\mu}_{\dot{s}} & =\mathbf{E}[\dot{\mathbf{s}}(k)] \\
& =f_{s} \mathbf{E}[\mathbf{s}(k)]-f_{s} \mathbf{E}[\mathbf{s}(k-1)] \\
& =\left(f_{s}-f_{s}\right) \boldsymbol{\mu}_{s}=0
\end{aligned}
$$


Onde $\boldsymbol{\mu}_{s}$ e $\boldsymbol{\mu}_{\dot{s}}$ são as médias do processo e de suas derivadas, respectivamente. A Eq. (5-10) mostra que o valores esperados de $\dot{\mathbf{s}}(k)$ são sempre zero, mesmo que $\boldsymbol{\mu}_{s}$ não seja. A partir desse resultado, o cálculo das variâncias é feito através de

$$
\begin{aligned}
\boldsymbol{\sigma}_{\dot{s}}^{2} & =\mathbf{E}\left[\left(\dot{\mathbf{s}}(k)-\boldsymbol{\mu}_{\dot{s}}\right)^{2}\right] \\
& =f_{s}^{2} \mathbf{E}\left[(\mathbf{s}(k)-\mathbf{s}(k-1))^{2}\right] \\
& =f_{s}^{2}\left\{\mathbf{E}\left[\mathbf{s}^{2}(k)\right]-2 \mathbf{E}[\mathbf{s}(k) \mathbf{s}(k-1)]+\mathbf{E}\left[\mathbf{s}^{2}(k-1)\right]\right\} \\
& =2 f_{s}^{2} \boldsymbol{\sigma}_{s}^{2}-2 f_{s} \mathbf{E}[\mathbf{s}(k) \mathbf{s}(k-1)],
\end{aligned}
$$

onde $\boldsymbol{\sigma}_{\dot{s}}^{2}$ são as variâncias do processo diferenciado. Se o sinal analisado for o ruído branco $\mathbf{v}(k)$, o termo $\mathbf{E}[\mathbf{s}(k) \mathbf{s}(k-1)]$ se torna zero, já que o ruído branco só é correlacionado com ele mesmo no instante de tempo. As variâncias da derivada dos ruídos são então definidas como

$$
\boldsymbol{\sigma}_{\dot{v}}^{2}=2 f_{s}^{2} \boldsymbol{\sigma}_{v}^{2}
$$

Isso significa que a relação entre as variâncias de um processo estacionário e sua derivada é dado pelo fator $2 f_{s}^{2}$. O cálculo das correlações das velocidades observadas, obtidas após uma derivação, é dado por

$$
\begin{aligned}
\mathbf{R}_{\dot{y} \dot{y}}\left(\tau_{k}\right)= & \mathbf{E}\left[\dot{\mathbf{y}}(k) \dot{\mathbf{y}}^{T}\left(k+\tau_{k}\right)\right] \\
= & \mathbf{E}\left[[\dot{\mathbf{q}}(k)+\dot{\mathbf{v}}(k)]\left[\dot{\mathbf{q}}^{T}\left(k+\tau_{k}\right)+\dot{\mathbf{v}}^{T}\left(k+\tau_{k}\right)\right]\right] \\
= & \mathbf{E}\left[\dot{\mathbf{q}}(k) \dot{\mathbf{q}}^{T}\left(k+\tau_{k}\right)\right]+\mathbf{E}\left[\dot{\mathbf{q}}(k) \dot{\mathbf{v}}^{T}\left(k+\tau_{k}\right)\right]+ \\
& +\mathbf{E}\left[\dot{\mathbf{v}}(k) \dot{\mathbf{q}}^{T}\left(k+\tau_{k}\right)\right]+\mathbf{E}\left[\dot{\mathbf{v}}(k) \dot{\mathbf{v}}^{T}\left(k+\tau_{k}\right)\right] \\
= & \mathbf{E}\left[\dot{\mathbf{q}}(k) \dot{\mathbf{q}}^{T}\left(k+\tau_{k}\right)\right]+\mathbf{E}\left[\dot{\mathbf{v}}(k) \dot{\mathbf{v}}^{T}\left(k+\tau_{k}\right)\right] \\
= & \mathbf{R}_{\dot{q} \dot{q}}\left(\tau_{k}\right)+\mathbf{R}_{\dot{v} \dot{v}}(\tau)
\end{aligned}
$$

Na quarta igualdade da eq.(18) utilizou-se novamente a hipótese de que $\dot{\mathbf{q}}(k)$ e $\dot{\mathbf{v}}(k)$ não serem correlacionados. No entanto, para que $\mathbf{E}\left[\dot{\mathbf{q}}(k) \dot{\mathbf{v}}^{T}\left(k+\tau_{k}\right)\right]$ e $\mathbf{E}\left[\dot{\mathbf{v}}(k) \dot{\mathbf{q}}^{T}\left(k+\tau_{k}\right)\right]$ sejam iguais a zero, mais amostras devem ser usadas, uma vez que as variâncias de $\dot{\mathbf{v}}(k)$ são maiores do que as variâncias de $\mathbf{v}(k)$.

Uma importante propriedade das correlações de processos estacionários (como por exemplo a resposta de um sistema excitado por um ruído branco) consiste nas relações entre suas derivadas. Em [43] e [44], mostra-se que a correlação de um processo estacionário e suas derivadas são relacionados através da equação

$$
\frac{d^{2}}{d \tau_{k}^{2}} \mathbf{R}_{s s}\left(\tau_{k}\right)=\mathbf{R}_{\ddot{s} s}\left(\tau_{k}\right)=-\mathbf{R}_{\dot{s} \dot{s}}\left(\tau_{k}\right),
$$


onde $\mathbf{s}(k)$ é novamente um processo estacionário qualquer. A relação acima é importante em análise modal quando os dados adquiridos durante um certo experimento precisam passar por processamentos de integração ou derivação. Esse é o caso, por exemplo, quando a aceleração de um sistema é medida por acelerômetros e se deseja calcular as funções de correlação do deslocamento.

A matriz de correlação da derivada do ruído pode ser aproximada por essa propriedade. Assim, a correlação da derivada do ruído é obtida através de

$$
\mathbf{R}_{\dot{v} \dot{v}}\left(\tau_{k}\right)=-\frac{d^{2} \mathbf{R}_{v v}\left(\tau_{k}\right)}{d \tau_{k}^{2}}=-\mathbf{G}_{v} \frac{d^{2} \delta\left(\tau_{k}\right)}{d \tau_{k}^{2}}
$$

A derivação do impulso unitário é conhecida, em processamento de sinais, como a função duoblet. Se o simples filtro mostrado na Eq. (5-9) for expandido para um de segunda ordem e aplicado a um impulso unitário, o resultado obtido é

$$
\frac{d^{2} \delta\left(\tau_{k}\right)}{d \tau_{k}^{2}}=f_{s}^{2}\left[-\delta\left(\tau_{k}-1 / f_{s}\right)+2 \delta\left(\tau_{k}\right)-\delta\left(\tau_{k}+1 / f_{s}\right)\right]
$$

Substituindo a Eq. (5-16) na Eq. (5-15) obtêm-se como resultado

$$
\mathbf{R}_{\dot{v} v}\left(\tau_{k}\right)= \begin{cases}-\mathbf{Q}_{v} \delta\left(\tau_{k}\right) f_{s}^{2} & , \tau_{k}=-1 \\ 2 \mathbf{Q}_{v} \delta\left(\tau_{k}\right) f_{s}^{2} & , \tau_{k}=0 \\ -\mathbf{Q}_{v} \delta\left(\tau_{k}\right) f_{s}^{2} & , \tau_{k}=1 \\ 0 & , \text { outros valores }\end{cases}
$$

Esse resultado mostra que após um processo de derivação o ruído distorce a correlação apenas nas amostras próximas de zero. Para este simples filtro, mais dois instantes de tempo são afetados pelo ruído, $\tau_{k}=-1$ e $\tau_{k}=1$.

\section{Exemplo 5.2}

Para exemplificar este resultado, uma simulação do mesmo sistema do exemplo anterior foi realizada. No entanto, obteve-se dessa vez o deslocamento do sistema. Em seguida, adicionou-se o ruído e o sinal resultante foi processado por um filtro de derivação. Uma vez obtido o respectivo sinal da velocidade, estimou-se a função de correlação. Para o caso onde não há ruído, a função de correlação é mostrado pela Figura 5.5a. Observa-se que esta função é a mesma obtida no exemplo anterior, já que o mesmo sistema e forçamento foram utilizados. Já a correlação do ruído, após o processo de derivação, apresenta uma forma diferente do exemplo anterior e pode ser vista pela Figura 5.5b. Percebe-se que o resultado é coerente com a Eq. (5-17). Nota-se que a ordem de grandeza desta função é muito maior quando comparado com a mostrada pela Figura 5.5b. Isso ocorre pelo fator $f_{s}^{2}$ na Eq. (5-17). Por fim, a correlação do sinal com ruído após o processo de derivação é mostrada na Figura 5.4c. 
Nota-se que essa correlação é igual a soma da correlação do sinal sem ruído com a correlação do ruído, como definido pela Eq. (5-13). A única parte permanentemente deformada pelo ruído consiste na região próxima de $\tau_{k}=0$.
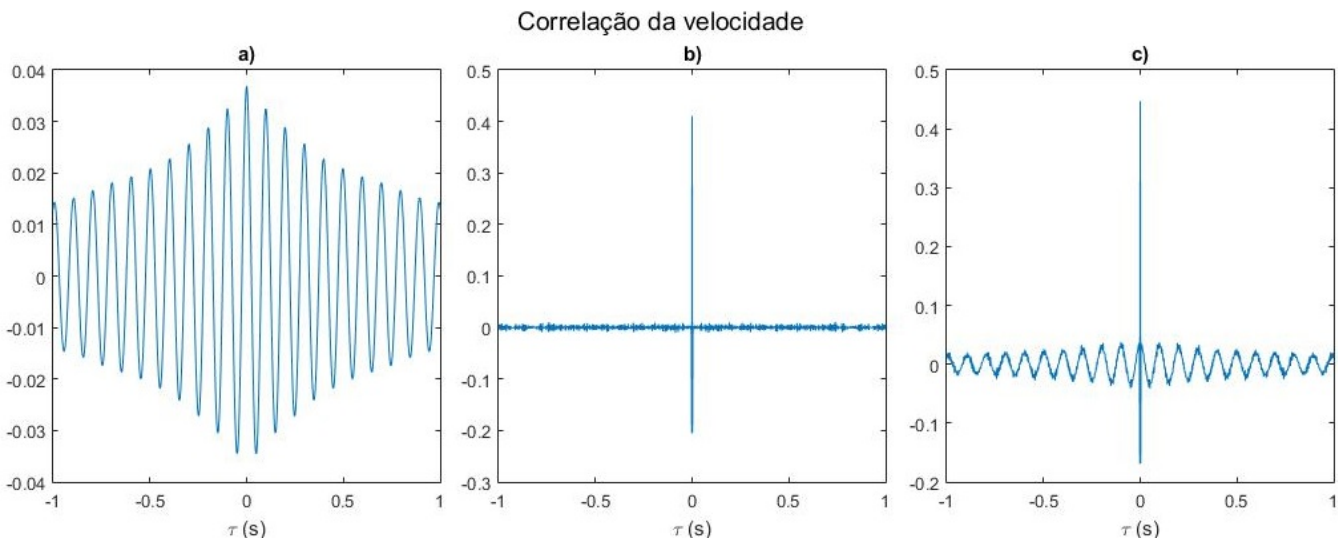

Figura 5.5: Correlações do sinal após um processo de derivação: a) resposta do sistema sem ruído, b) ruído e c)resposta do sistema com ruído

A derivada do sinal mostrados na Figura 5.5 foi realizada pelo simples filtro apresentado na Eq. (5-9). No entanto, referências como [26][27][32] propõem filtros mais complexos que resultam em menores erros. As correlações do ruído após a utilização destes filtros são mostrados na Figura 5.6. Percebese que a diferença entre os resultados é pequena e que em todos eles os valores relevantes estão somente próximo a zero. (Fim do exemplo)
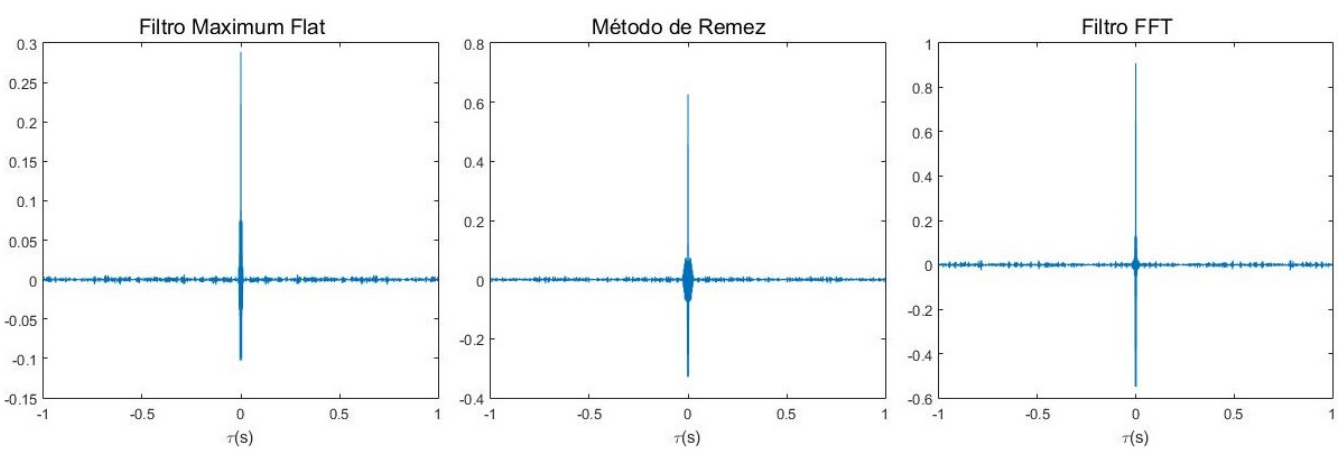

Figura 5.6: Correlações do ruído após um processo de derivação com diferentes filtros

\subsection{3}

\section{Correlação da integral da resposta do sistema com ruído}

Seguindo o mesmo procedimento apresentado nas seções anteriores, a função de correlação da velocidade é novamente calculada. No entanto, partese desta vez do sinal de aceleração do sistema. O ruído é então adicionado a 
esse sinal e em seguida o processo de integração é realizado. Só então a função de correlação é calculada. Seja $\ddot{\mathbf{y}}(k)$ o sinal resultante da soma da aceleração do sistema com um ruído, como mostrado pela Eq. (5-3) e repetida abaixo por conveniência,

$$
\ddot{\mathbf{y}}(k)=\ddot{\mathbf{x}}(k)+\mathbf{v}(k) .
$$

Ao realizar o processo de integração deste sinal, obtêm-se

$$
\dot{\mathbf{y}}(k)=\dot{\mathbf{x}}(k)+\mathbf{V}(k),
$$

onde o sinal $\mathbf{V}(k) \in \mathbb{R}^{n}$ corresponde a integral do ruído branco, que, se modelado com distribuição gaussiana, corresponde a um processo chamado de Wiener (Brownian Motion). No entanto, como as integrais são aproximadas nessa dissertação por filtros, o sinal resultante não pode ser classificado como um processo de Wiener propriamente dito. Quando visto no domínio da frequência, a integração consiste em dividir o espectro por $i \omega$. Isso significa que, ao integrar, as frequência mais baixas são potencializadas enquanto as mais altas são atenuadas. Ao dividir o espectro por $\omega$, um problema numérico surge ao se aproximar de DC (frequência zero). Para contornar este problema, é comum, em processamento de sinais, utilizar filtros passa alta antes da integração. A frequência de corte deste filtro precisa ser baixa o suficiente para não interferir nos resultados.

As correlações dos sinais de velocidade definidas pela Eq. (5-19) são calculadas através de equação

$$
\begin{aligned}
\mathbf{R}_{\dot{y} \dot{y}}\left(\tau_{k}\right)= & \mathbf{E}\left[\dot{\mathbf{y}}(k) \dot{\mathbf{y}}^{T}\left(k+\tau_{k}\right)\right] \\
= & \mathbf{E}\left[[\dot{\mathbf{x}}(k)+\mathbf{V}(k)]\left[\dot{\mathbf{x}}^{T}\left(k+\tau_{k}\right)+\mathbf{V}^{T}\left(k+\tau_{k}\right)\right]\right] \\
= & \mathbf{E}\left[\dot{\mathbf{x}}(k) \dot{\mathbf{x}}^{T}\left(k+\tau_{k}\right)\right]+\mathbf{E}\left[\dot{\mathbf{x}}(k) \mathbf{V}^{T}\left(k+\tau_{k}\right)\right]+ \\
& +\mathbf{E}\left[\mathbf{V}(k) \dot{\mathbf{x}}^{T}\left(k+\tau_{k}\right)\right]+\mathbf{E}\left[\mathbf{V}(k) \mathbf{V}^{T}\left(k+\tau_{k}\right)\right] \\
= & \mathbf{E}\left[\dot{\mathbf{x}}(k) \dot{\mathbf{x}}^{T}\left(k+\tau_{k}\right)\right]+\mathbf{E}\left[\mathbf{V}(k) \mathbf{V}^{T}\left(k+\tau_{k}\right)\right] \\
= & \mathbf{R}_{\dot{x} \dot{x}}\left(\tau_{k}\right)+\mathbf{R}_{V V}\left(\tau_{k}\right) .
\end{aligned}
$$

Mais uma vez utilizou-se a hipótese dos sinais $\dot{\mathbf{x}}(k)$ e $\mathbf{V}(k)$ não serem correlacionados.

\section{Exemplo 5.3}

Este exemplo utiliza o mesmo sistema e forçamento dos exemplos anteriores. Para realizar a integração dos sinais, quatro diferentes filtro foram adotados: regra do trapézio, passa baixa de primeira ordem, IIR de oitava ordem e um filtro FFT [26][27]. O resultado das correlações com o filtro da regra do trapézio é mostradas na Figura 5.7. Através da Figura 5.7a, observa-se que a correlação 
do sinal da velocidade sem ruído é a mesma obtida nas Figuras 5.3 a e 5.5a. Esse fato valida o filtro como um operador de integração. Já a Figura $5.7 b$ ilustra a correlação do ruído após a integração. Nota-se que nesta função as maiores magnitudes continuam próximas a zero, no entanto em banda de $\tau_{k}$ maior. Ao olhar a escala deste gráfico, percebe-se que a ordem de grandeza é bastante inferior quando comparada à correlação da velocidade. Isso significa que, ao contrário da derivação, o processo de integração diminuí a variância do ruído. Assim, a deformação causada pelo ruído é pequena e não pode se observada pela Figura 5.7c.
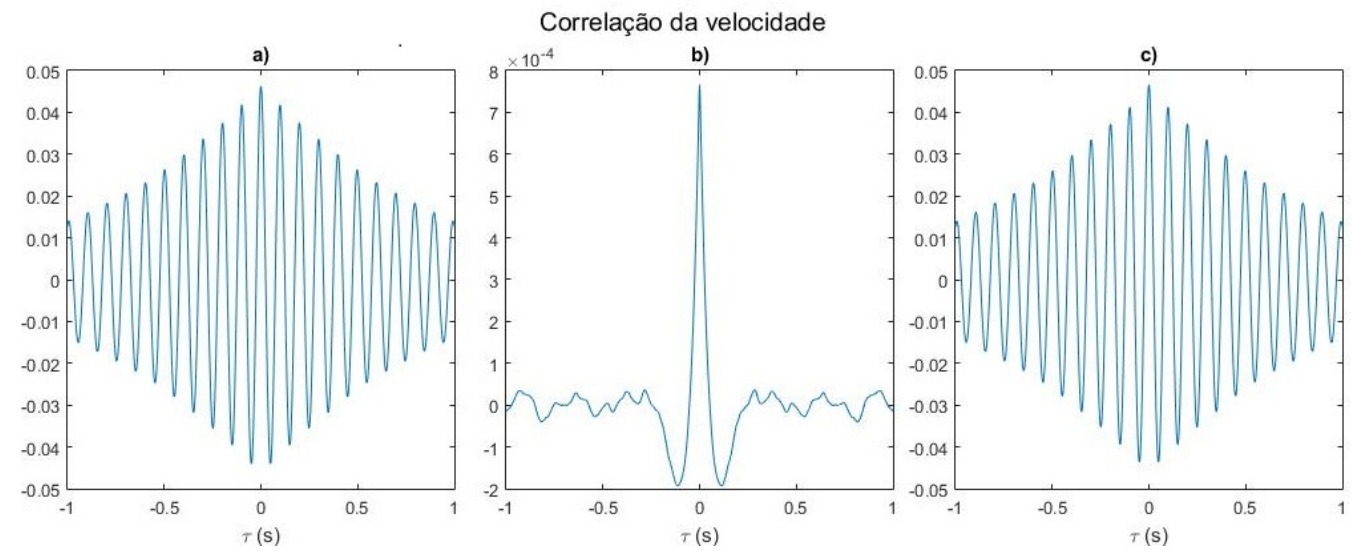

Figura 5.7: Correlações do sinal após o processo de integração: a)resposta do sistema sem ruído, b) ruído e c)resposta do sistema com ruído

O filtro que representa a regra do trapézio não é um filtro aconselhado por não ser muito preciso quando a frequência de aquisição é baixa. Por isso, os resultados das correlações do sinal de ruído utilizando os outros filtros citados acima são mostrados na Figura 5.8. Percebe-se que os resultados são semelhantes, apenas com algumas variações de amplitude. Como explicado anteriormente, todos os sinais passaram por um filtro passa alta antes do filtro de integração. A frequência de corte deste filtro foi escolhido como $1 \mathrm{~Hz}$, que corresponde a um valor bem inferior que a frequência natural do sistema, que é de $10 \mathrm{~Hz}$. (Fim do exemplo)

\section{2}

\section{Conclusões}

Após uma análise da distorção que o ruído pode causar nas funções de correlação de um sinal, algumas observações podem ser feitas.

- As distorções causadas pelo ruído são as próprias funções de correlação dos ruídos. 

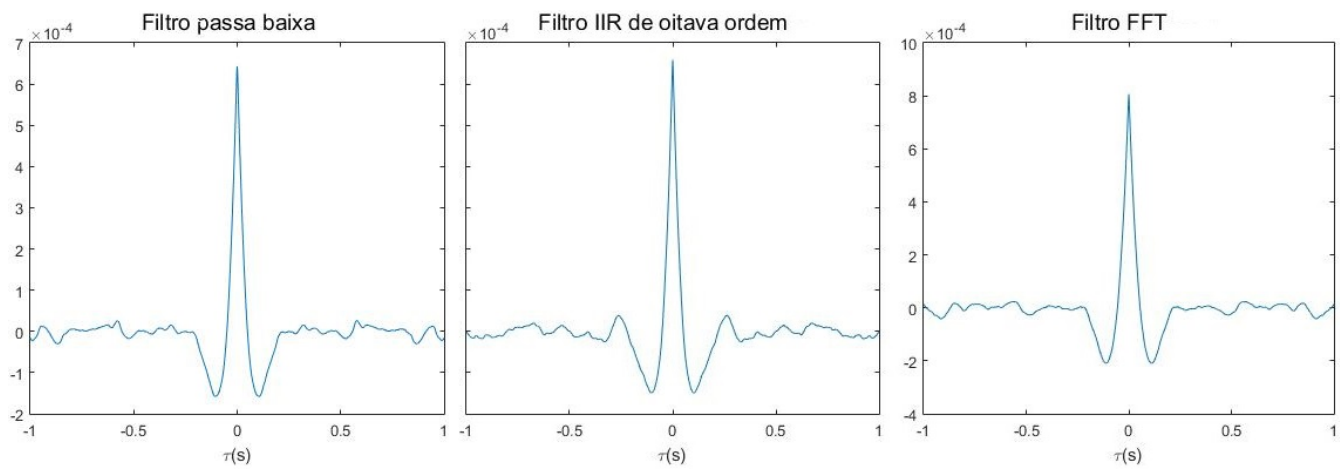

Figura 5.8: Correlações do ruído após um processo de integração com diferentes filtros

- As correlações do ruído possuem valores significativos apenas em regiões próximas a zero, independente se o sinal passou por um processo de integração ou derivação.

- Quando o sinal passa por um processo de derivação, a variância tende a ser aumentada, fazendo com que a distorção causada pelo ruído nas correlações sejam mais relevantes. O processo inverso ocorre para no caso da integração. No entanto, para a integração, a banda de $\tau_{k}$ onde a função de correlação do ruído tem valores diferentes de zero é maior.

Para futuros trabalhos, é necessário que um estudo mais aprofundado das propriedades e classificações dos sinais após integrações e derivações seja feito. 


\section{Métodos clássicos de identificação no domínio do tempo}

Quando a análise modal é aplicada experimentalmente, o principal objetivo consiste em quantificar os parâmetros modais do sistema através de testes de vibrações. Nesta dissertação, a identificação destes parâmetros será realizada através da análise modal operacional, onde apenas as respostas de um sistema são utilizadas. Além disso, somente os métodos no domínio do tempo serão discutidos. Para que eles possam ser compreendidos e corretamente aplicados, todos os resultados obtidos nos capítulos anteriores precisam ser utilizados.

No capítulo 2, mostrou-se que as IRFs e as FRFs definiam um sistema e podiam ser escritas em função dos parâmetros modais. No domínio do tempo, mostrou-se que as IRFs podiam ser expressas como um somatório de decaimentos exponenciais, onde cada termo era definido pelos parâmetros de apenas um respectivo modo. No capítulo 3, analisou-se o comportamento dos mesmos tipos de sistemas, no entanto quando submetidos a forçamentos aleatórios. Utilizando algumas hipóteses para esse forçamento e para o sistema, mostrou-se que as funções de correlação da resposta possuem um estrutura semelhante às das IRFs. Isso significa que as correlações também podem ser escritas como um somatório de decaimentos exponenciais e que cada termo também é definido por um dos respectivos modos do sistema. O capítulo 4 foi responsável por introduzir os modelos de espaço de estado e ARMA que serão utilizados pelos métodos de identificação deste capítulo. Ambos os modelos foram definidos de forma discreta, visto que este é o tipo de sinal que se obtêm durante um experimentos. No capítulo 5, foi feito um estudo de como possíveis ruídos podem distorcer as funções de correlação. Esse estudo é levado em consideração quando se analisa a sensibilidade de cada um dos método em relação aos ruídos.

Neste capítulo, três diferentes métodos de identificação no domínio do tempo serão apresentados. Os métodos AR/PR (auto regressive / Poly Reference), ERA (Eigensystem Realization Algorithm) e o de subespaço estocástico foram escolhidos por serem os mais aplicados atualmente. Para cada um deles, um estrutura real foi utilizada ilustrar o procedimento de identificação do ponto de vista experimental, assim como validar a implementação dos algoríti- 
mos. Esses exemplos permitem também uma discussão de como os resultados devem ser analisados.

\section{1}

\section{Auto regressive / Poly Reference}

Considerado um dos mais simples métodos de identificação, o AR/PR é assim denominado pois utiliza uma modelagem AR (auto regressive) das funções de correlações e identifica as respectivas matrizes utilizando uma técnica PR (poly reference). Esse método utiliza os resultados obtidos na seção 4.2 , onde mostrou-se que a resposta livre de um sistema pode ser modelado pela parte $\mathrm{AR}$ e que os respectivos parâmetros modais podem ser extraídos utilizando uma matriz de acompanhamento ( $\mathbf{A}_{d}$ definida na Eq. (4-38)).

Como mostrado no capítulo 3 , as funções de correlação possuem o mesmo formato das respostas livres de um sistema. Por isso, o mesmo modelo AR pode ser aplicado ambas funções com apenas pequenas modificações. Através da Eq. (4-36), o modelo AR foi utilizado para descrever o decaimento exponencial da resposta livre de um sistema. Esse modelo é repetido a seguir utilizando $N$ matrizes AR. Além disso, as modificações necessárias para utilizá-lo em OMA também são mostradas, isso é, um adaptação para utilizar as funções de correlação.

$$
\begin{array}{r}
\mathbf{y}(k)-\mathbf{A}_{1} \mathbf{y}(k-1)+\cdots+\mathbf{A}_{N} \mathbf{y}(k-N)=0 \\
\Downarrow \\
\mathbf{R}_{y y}\left(\tau_{k}\right)-\mathbf{A}_{1} \mathbf{R}_{y y}\left(\tau_{k}-1\right)+\cdots+\mathbf{A}_{N} \mathbf{R}_{y y}\left(\tau_{k}-N\right)=0
\end{array}
$$

Na equação acima, $\mathbf{y}(k) \in \mathbb{R}^{n_{o}}$ é o vetor das $n_{o}$ respostas medidas nos instantes de tempo $t_{k}\left(t_{k}=k \Delta t\right)$. Já as matrizes $\mathbf{R}_{y y}\left(\tau_{k}\right) \in \mathbb{R}^{n_{o} \times n_{o}}$ representam matrizes de correlação dessas respostas, calculadas com uma defasagem de amostras $\tau_{k}$ entre os sinais através da equação

$$
\mathbf{R}_{y y}\left(\tau_{k}\right)=\mathbf{E}\left[\mathbf{y}(k) \mathbf{y}^{T}\left(k+\tau_{k}\right)\right]
$$

A única mudança feita na Eq. (6-1) foi a transformação do vetor $\mathbf{y}(k)$ em uma matriz $\mathbf{R}_{y y}\left(\tau_{k}\right)$. No entanto, as matrizes $\mathbf{A}_{\mathbf{r}}, r=1, \ldots, N$ do modelo AR continuam tendo o mesmo formato. Por isso, o mesmo procedimento utilizado para obter os parâmetros modais apresentado na seção 4.2 pode continuar sendo usado a seguir.

Para que os parâmetros modais sejam identificados basta então que todas as matrizes do modelo AR sejam conhecidas. A técnica utilizada para essa identificação é chamada de PR (poly reference) e será apresentada a seguir. 
Para isso, é necessário que uma arrumação matricial chamada de Hankel sejam definidas. Uma matriz de Hankel é uma matriz de blocos na qual cada elemento (vetor ou matriz) localizado diretamente a esquerda ou abaixo tenha um acréscimo de tempo. Para o método AR/PR, duas matrizes de Hankel precisam ser construídas a partir das matrizes de correlações. São elas

$$
\mathcal{R}_{1 \mid N}=\left[\begin{array}{cccc}
\mathbf{R}_{y y}(1) & \mathbf{R}_{y y}(2) & \ldots & \mathbf{R}_{y y}\left(N_{s}-N\right) \\
\mathbf{R}_{y y}(2) & \mathbf{R}_{y y}(3) & \ldots & \mathbf{R}_{y y}\left(N_{s}-N+1\right) \\
\vdots & \vdots & \ddots & \vdots \\
\mathbf{R}_{y y}(N) & \mathbf{R}_{y y}(N+1) & \ldots & \mathbf{R}_{y y}\left(N_{s}-1\right) .
\end{array}\right]
$$

$\mathrm{e}$

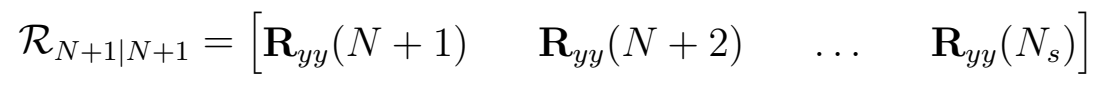

Os subscritos 1| $N$ na Eq. (6-3) e $N+1 \mid N+1$ na Eq. (6-4) indicam a defasagem de amostras $\tau_{k}$ da primeira e última correlação utilizada na primeira coluna das matrizes de Hankel. Isso significa que, na Eq. (6-3), $N$ blocos de linhas foram utilizados e que as respectivas correlações na primeira coluna foram calculadas utilizando uma defasagem $\tau_{k}$ variando de 1 a $N$. Já para a Eq. (64), apenas um bloco de linhas foi utilizado. A correlação utilizada na primeira coluna tem a defasagem $\tau_{k}$ igual a $N+1$. O número de blocos de linhas na Eq. (6-3) é igual a quantidade de matrizes AR utilizadas no modelo da Eq. (6-1). Esse número precisa ser escolhida pelo analista e está relacionada com a quantidade de parâmetros modais necessários para uma boa caracterização do sistema, que a princípio não é conhecido.

Como mostrado pelas Eqs. (6-3,/6-4), as matrizes $\mathcal{R}_{1 \mid N}$ e $\mathcal{R}_{N+1 \mid N+1}$ contém $N_{s}-N$ blocos de colunas. O valor de $N_{s}$ está relacionado com a quantidade de diferentes defasagens de tempo utilizadas para calcular as matrizes de correlação. Normalmente, esse número precisa ser grande, fazendo com que as matrizes de Hankel tenham um forma retangular acentuada. Uma melhor análise da influência desse parâmetro será feita mais adiante. As dimensões finais das duas matrizes de Hankel são: $N n_{o} \times\left(N_{s}-N\right) n_{o}$ para a matriz $\mathcal{R}_{1 \mid N}$ e $n_{o} \times\left(N_{s}-N\right) n_{o}$ para $\mathcal{R}_{N+1 \mid N+1}$.

Utilizando as matrizes de Hankel, o modelo AR mostrado na Eq. (6-1) pode ser reescrito para $N_{s}-N$ valores de $\tau_{k}$ utilizando a equação

$$
\mathcal{R}_{N+1 \mid N+1}-\mathcal{A R}_{1 \mid N}=0 \Leftrightarrow \mathcal{A R}_{1 \mid N}=\mathcal{R}_{N+1 \mid N+1},
$$

onde $\mathcal{A}$ é definida como sendo

$$
\mathcal{A}=\left[\begin{array}{llll}
\mathbf{A}_{N} & \mathbf{A}_{N-1} & \ldots & \mathbf{A}_{1}
\end{array}\right]
$$


Ao calcular a transposta da Eq. (6-5), um problema super determinado é criado e pode ser resolvido através de métodos de mínimos quadrados ou decomposição por valores singulares. A estimativa $\hat{\mathcal{A}}$ para a matriz $\mathcal{A}$ pode então ser feita através da equação

$$
\begin{gathered}
\mathcal{R}_{1 \mid N}^{T} \mathcal{A}^{T}=\mathcal{R}_{N+1 \mid N+1}^{T} \\
\Downarrow \\
\hat{\mathcal{A}}=\mathcal{R}_{N+1 \mid N+1} \mathcal{R}_{1 \mid N}^{\dagger},
\end{gathered}
$$

onde $\mathcal{R}_{1 \mid N}^{\dagger}$ é a inversa generalizada da matriz $\mathcal{R}_{1 \mid N}$. Como as matrizes de correlação têm dimensão $n_{o} \times n_{o}$, o problema só é super determinado quando a quantidade de blocos de linhas e colunas das matrizes de Hankel respeitam a seguinte relação $\left(N_{s}-N\right)>N$. No entanto, como existe sempre uma certa quantidade de ruído nos sinais e erros no cálculo das correlações, a estimativa da matriz $\mathcal{A}$ só é bem feita quando o sistema é exageradamente super determinado. Isso significa que as dimensões das matrizes de Hankel devem respeitar a condição

$$
\left(N_{s}-N\right) \gg N
$$

Uma fez estimado $\hat{\mathcal{A}}$, a matriz de acompanhamento $\mathbf{A}_{d}$ do modelo AR pode ser construída utilizando a Eq. (4-38), isto é

$$
\mathbf{A}_{d}=\left[\begin{array}{ccccc}
0 & \mathbf{I} & 0 & \ldots & 0 \\
0 & 0 & \mathbf{I} & \ldots & 0 \\
\vdots & \vdots & \vdots & \ddots & \vdots \\
0 & 0 & 0 & \ldots & \mathbf{I} \\
\mathbf{A}_{N} & \mathbf{A}_{N-1} & \mathbf{A}_{N-2} & \ldots & \mathbf{A}_{1}
\end{array}\right]
$$

Percebe-se que o último bloco de linhas nessa matriz corresponde a própria matriz $\mathcal{A}$. Os parâmetros modais são então obtidos através dos autovalores e autovetores de $\mathbf{A}_{d}$ (resultado mostrado na seção 4.2). Como a matriz de acompanhamento têm dimensão $n_{o} N \times n_{o} N$, o seguinte problema de autovalor é criado

$$
\mathbf{A}_{d} \boldsymbol{\psi}_{i, d}=\mu_{i} \boldsymbol{\psi}_{i, d}, \quad i=1, \ldots, n_{o} N
$$

Os parâmetros de frequência natural e fator de amortecimento são obtidos da mesma forma como mostrado pela Eq. (4-32), repetida abaixo

$$
\mu_{i}=\frac{\log \left(\lambda_{i}\right)}{\Delta t}, \quad f_{i}=\frac{\left|\mu_{i}\right|}{2 \pi}, \quad \zeta_{i}=\frac{-\operatorname{Re}\left(\mu_{i}\right)}{\left|\mu_{i}\right|}, \quad i=1, \ldots, n_{o} N
$$

onde $\mu_{i}$ correspondem os polos do sistema associados ao modelo contínuo, $f_{i}$ as frequências naturais e $\zeta_{i}$ os fatores de amortecimento. Os modos do sistema 
são obtidos pela últimas $n_{o}$ linhas do autovetor $\boldsymbol{\psi}_{i, d}$. Como a Eq. (6-10) resulta em $n_{o} N$ autovalores e autovetores, é preciso que este número seja maior que a quantidades de modos necessários para caracterizar bem o sistema. Como esse número de modos não é conhecido a princípio, a identificação deve ser realizada diversas vezes para valores crescentes de $N$. As respectivas frequências natural obtidas nesse processo podem ser marcadas juntas em um mesmo gráfico, construindo assim um diagrama de estabilidade. Uma melhor descrição é feita a seguir utilizando os resultados do exemplo abaixo.

\section{Exemplo 6.1}

Para exemplificar este método de identificação, uma estrutura que simula um prédio de dois andares foi utilizada [45]. Uma foto e o desenho desta estrutura é mostra a Figura 6.1. Dois sensores a laser foram utilizados para medir os deslocamentos, em ambos os andares, e de forma unidirecional. Para excitar o sistema, foi introduzido um vento em sua direção. Na prática, esse tipo de força é comumente encontrado, como por exemplo no caso de grandes prédios, pontes, antenas, etc. A força gerada pela interação fluido-estrutura é considerada aleatória e não pode ser medida de forma precisa, e por isso apenas a análise modal operacional pode ser aplicada. Para aumentar a magnitude da força gerada pelo vento, uma folha plástica foi acrescentada à estrutura para simular as paredes de um prédio.

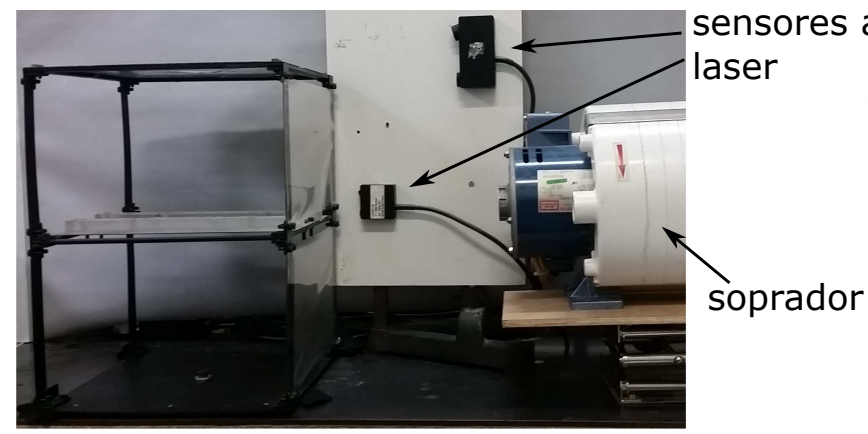

a)

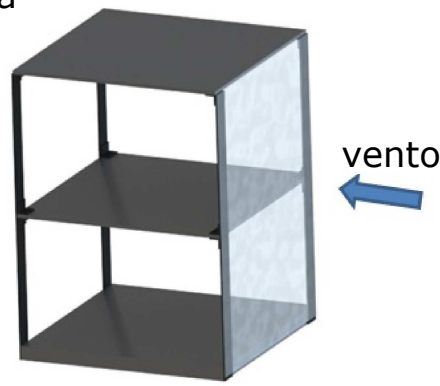

b)

Figura 6.1: Modelo de uma prédio de dois andares forçado pelo vento

No planejamento de um experimento, a banda de frequência a ser analisada deve ser sempre escolhida primeiro. A definição deste parâmetro é geralmente feita com base em um conhecimento prévio da estrutura, como por exemplo por simulações numéricas. Para este modelo em questão, a banda de frequência escolhida foi de 0 a $15 \mathrm{~Hz}$. Em seguida, a frequência de aquisição foi definida com um valor de $150 \mathrm{~Hz}$, para resultar em fator de oversampling (frequência de aquisição dividida pela máxima frequência analisada) igual a 10. 
O número de amostras necessárias para a identificação depende da quantidade de parâmetros modais do sistema (número de matrizes $A R$ ) e a relação sinal/ruído. Essa afirmação está relacionada com a Eq. (6-8), visto que o número de colunas na matrizes de Hankel esta relacionado com a quantidade de amostras (númeor de correlações que se pode estimar). Caso a identificação não resulte em bons valores, o número de amostras deve ser novamente avaliado e provavelmente aumentado. Para este exemplo, 204.600 amostras foram utilizadas. O calculo das matrizes de correlações foi feito para $\tau_{k}$ variando entre 0 e 1024.

Utilizando o procedimento descrito nessa seção, os parâmetros modais da bancada foram identificados diversas vezes para diferentes números de blocos de linhas $N$ na matriz de Hankel $\mathcal{R}_{1 \mid N}$. Essa variação ocorreu para valores entre de 1 a 20, e é equivalente ao mesmo aumento da quantidade de matrizes AR no modelo da Eq. (6-3). Para cada polo identificado (autovalor da Eq. (6-10)), a respectiva frequência natural (calculada pela Eq. (6-11)) foi marcada na Figura 6.2 em função do valor de $N$ utilizado (eixo vertical). Como descrito anteriormente, essa figura é chamada e diagrama de estabilização. Para esse exemplo, três diferentes classificações para os polos do sistema foram utilizadas. Os polos estáveis são aqueles cujas frequências naturais e fatores de amortecimento têm valores iguais aos já identificados utilizando menos uma matriz AR (menos um bloco de linha na matriz de Hankel). Já os polos classificados como "frequência estável", são aqueles onde apenas as frequências naturais têm valores iguais ao do modelo anterior. Caso nem as respectivas frequências naturais nem os fatores de amortecimento tenham valores iguais, os polos são classificados como instáveis.

Como pode ser observado na Figura 6.2, os polos estáveis tendem a criar uma linha vertical, o que indicam que esses polos estão realmente relacionados com a estrutura. Independente da quantidade de matrizes AR utilizadas no modelos, os parâmetros modais da estrutura devem ser sempre identificados. Já os polos instáveis não apresentam esse mesmo comportamento. Isso porque eles estão vinculados a possíveis erros numéricos e aos ruído do sinal. Por isso, para cada novo valor de $N$, polos com diferentes valores são obtidos. Ao fundo da figura, os valores singulares das matrizes $\mathbf{G}_{y y}$ (densidades espectrais de potência das respostas do sistema) são plotados para cada valor de $\omega$. Isso serve pra comparar a posição dos picos nos espectros com as linhas estáveis dos polos. Neste exemplo, os picos dos espectros e as linha estáveis se coincidiram, o que indica uma boa identificação dos parâmetros. Os valore finais das frequências naturais e fatores de amortecimento para essa duas linha estáveis são mostradas na tabela 6.1 
Tabela 6.1: Parâmetros modais identificados pelo método AR/PR

\begin{tabular}{ccc}
\hline Modo & Frequência Natural [Hz] & Fator de Amortecimento [\%] \\
$1^{\mathrm{o}}$ & 3.381 & 0.93 \\
$2^{\mathrm{o}}$ & 8.624 & 0.45 \\
\hline
\end{tabular}

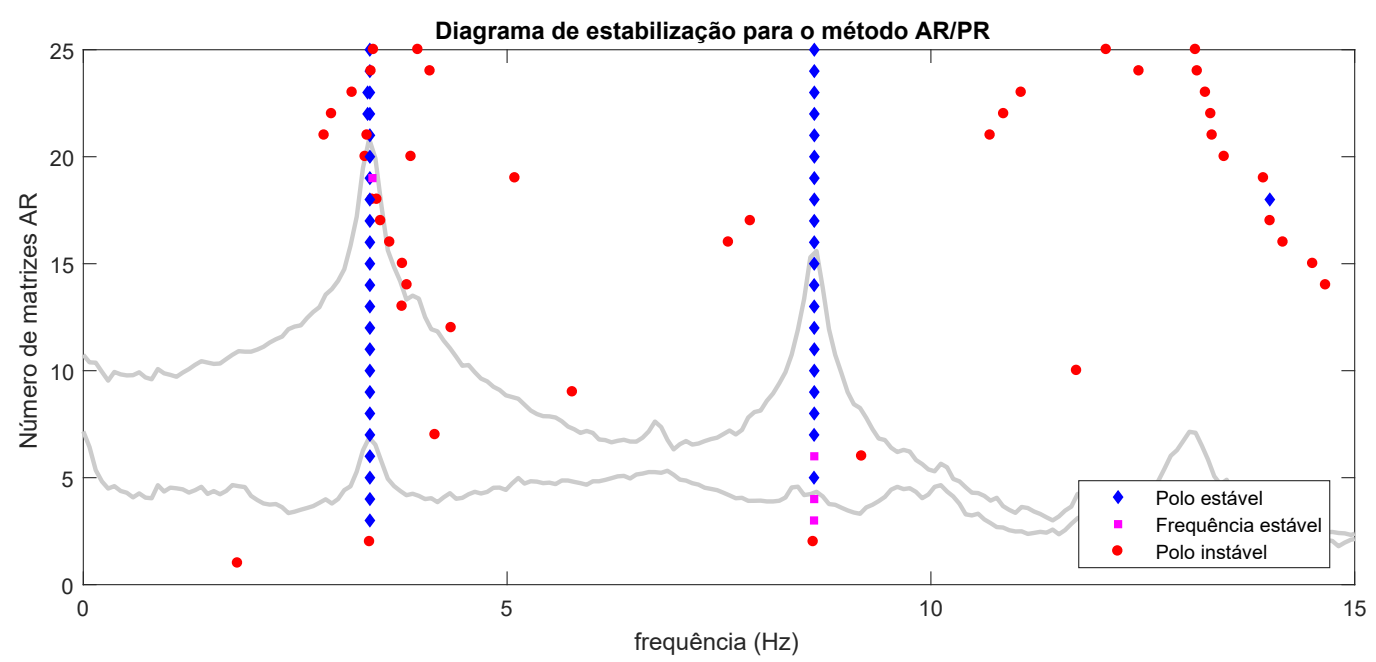

Figura 6.2: Diagrama de estabilização do modelo do prédio utilizando o método de identificação $\mathrm{AR} / \mathrm{PR}$

Por último, o formato do modos associados aos polos das linha estáveis são mostrados pela Figura 6.3. Esses modos são iguais ao de um modelo de dois graus de liberdade, e por isso são considerados realistas. Assim, a identificação de todos os parâmetros modais pelo método $A R / P R$ foi considerada boa. (Fim do exemplo)
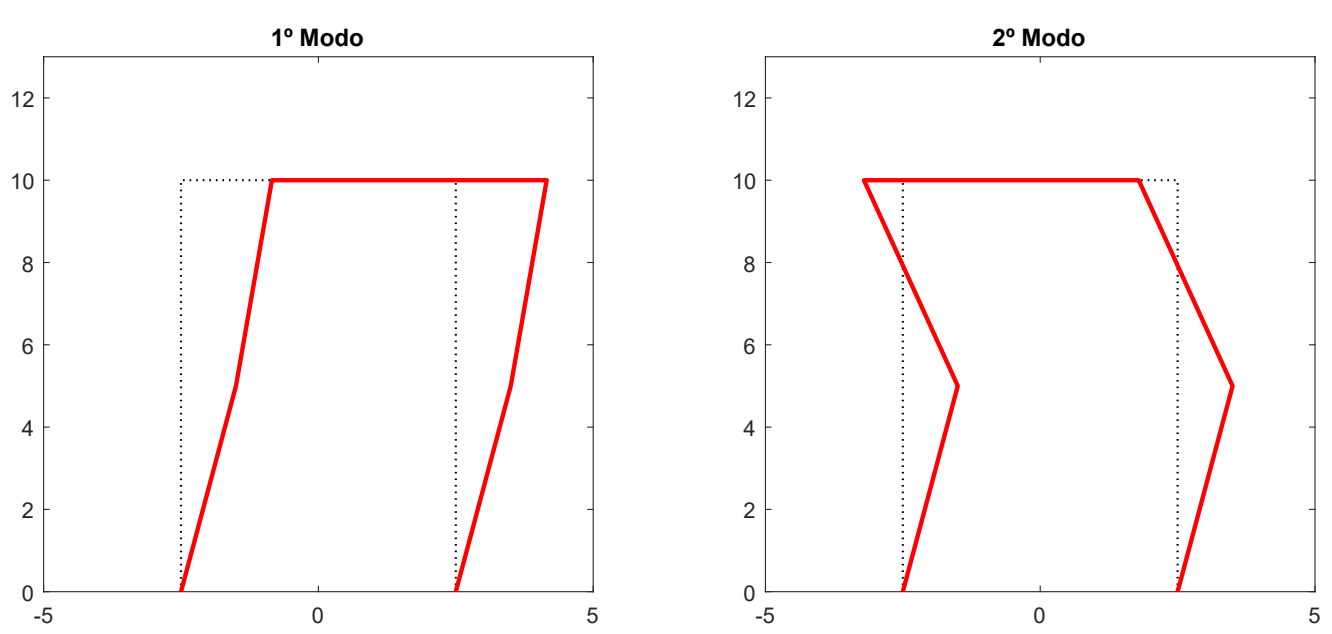

Figura 6.3: Formato dos modos de vibração do modelo do prédio identificados pelo método $\mathrm{AR} / \mathrm{PR}$ 


\section{2}

\section{Eigensystem Realization Algorithm}

O método de Eigensystem Realization Algorithm (ERA) foi desenvolvido nos anos oitenta por J.N. Jang e R.S. Pappa [46, 47, 48] a partir da teoria de controle. Para uma revisão completa do método, a referência [37] é indicada.

Originalmente, esse método foi desenvolvido para análise modal experimental (EMA) e por isso as funções de resposta ao impulso eram utilizadas. Para a utilização deste método em OMA, basta que essas funções sejam substituídas pelas de correlação da resposta do sistema. O método inicia novamente com a construção de duas matrizes de Hankel. Neste caso, essas matrizes são definidas como

$$
\mathcal{R}_{1 \mid N}=\left[\begin{array}{cccc}
\mathbf{R}_{y y}(1) & \mathbf{R}_{y y}(2) & \ldots & \mathbf{R}_{y y}\left(N_{s}-N\right) \\
\mathbf{R}_{y y}(2) & \mathbf{R}_{y y}(3) & \ldots & \mathbf{R}_{y y}\left(N_{s}-N+1\right) \\
\vdots & \vdots & \ddots & \vdots \\
\mathbf{R}_{y y}(N) & \mathbf{R}_{y y}(N+1) & \ldots & \mathbf{R}_{y y}\left(N_{s}-1\right) .
\end{array}\right]
$$

e

$$
\mathcal{R}_{2 \mid N+1}=\left[\begin{array}{cccc}
\mathbf{R}_{y y}(2) & \mathbf{R}_{y y}(3) & \ldots & \mathbf{R}_{y y}\left(N_{s}-N+1\right) \\
\mathbf{R}_{y y}(3) & \mathbf{R}_{y y}(4) & \ldots & \mathbf{R}_{y y}\left(N_{s}-N+2\right) \\
\vdots & \vdots & \ddots & \vdots \\
\mathbf{R}_{y y}(N+1) & \mathbf{R}_{y y}(N+2) & \ldots & \mathbf{R}_{y y}\left(N_{s}\right) .
\end{array}\right]
$$

Desta vez, percebe-se que ambas as matrizes têm mesma dimensão $N n_{o} \times$ $\left(N_{s}-N\right) n_{o}$. A única diferença consiste no valor de $\tau_{k}$ da primeira correlação na primeira coluna, fazendo com que toda a matriz $\mathcal{R}_{2 \mid N}$ esteja uma amostra a frente de $\mathcal{R}_{1 \mid N-1}$. Como mostrado na seção 4.1.4, as funções de correlação podem ser escritas em termos das matrizes de espaço de estado através da Eq. (4-27), repetida abaixo.

$$
\mathbf{R}_{y y}\left(\tau_{k}\right)=\mathbf{C A}^{\tau_{k}-1} \mathbf{G} .
$$

Utilizando essa expressão, as duas matrizes de Hankel podem ser reescritas na forma

$$
\mathcal{R}_{1 \mid N-1}=\boldsymbol{\Gamma} \mathcal{O} ; \quad \mathcal{R}_{2 \mid N}=\Gamma \mathbf{A} \mathcal{O},
$$

onde,

$$
\boldsymbol{\Gamma}=\left[\begin{array}{c}
\mathbf{C} \\
\mathbf{C A} \\
\mathbf{C A}^{2} \\
\vdots \\
\mathbf{C A}^{N-1}
\end{array}\right] ; \quad \mathcal{O}=\left[\begin{array}{lllll}
\mathbf{G} & \mathbf{A G} & \mathbf{A}^{2} \mathbf{G} & \ldots & \mathbf{A}^{N_{s}-N-1} \mathbf{G}
\end{array}\right]
$$


A matriz $\Gamma$ é chamada de matriz de observabilidade do sistema, enquanto a matriz $\mathcal{O}$ pode ser pensada como a matriz de controlabilidade revertida do sistema estocástico. A principal ideia do ERA consiste em realizar a decomposição por valores singulares (SVD) da matriz $\mathcal{R}_{1 \mid N-1}$ para depois estimar as matrizes de observabilidade e controlabilidade. A SVD da matriz $\mathcal{R}_{1 \mid N-1}$ pode então ser expressa por

$$
\mathcal{R}_{1 \mid N-1}=\mathbf{U S V}^{T}
$$

onde $\mathbf{U}$ e $\mathbf{V}$ são matrizes que contém os vetores singulares arrumados em colunas e $\mathbf{S}$ uma matriz diagonal com os respectivos valores singulares. Como a matriz A do modelo de espaço de estado tem posto igual a $2 n$ (duas vezes a ordem do sistema), tanto a matriz de observabilidade quanto a matriz de controlabilidade também terão posto $2 n$. Como $\mathcal{R}_{1 \mid N-1}$ é definida pela Eq. (615) como o produto destas duas matrizes, ela também terá posto igual a $2 n$ e consequentemente esta mesma quantidade de valores singulares não nulos. Na prática, esse valor pode ser um pouco maior por conta dos ruídos nos sinais de resposta do sistema. A definição de quantos valores singulares estão realmente relacionados ao sistema e quantos estão relacionados aos ruídos pode ser feita através de um diagrama de estabilização semelhante ao apresentado no Exemplo 6.1. A Eq. (6-17) pode então ser reescrita de forma compacta através de

$$
\mathcal{R}_{1 \mid N-1}=\mathbf{U}_{n} \mathbf{S}_{n} \mathbf{V}_{n}^{T},
$$

onde $\mathbf{U}_{n}$ e $\mathbf{V}_{n}$ são as matrizes contendo as $n$ primeiras colunas de $\mathbf{U}$ e $\mathbf{V}$, respectivamente. A matriz $\mathbf{S}_{n}$ é uma matriz diagonal contendo apenas os $n$ primeiros valores singulares da matriz $\mathbf{S}$.

Utilizando a Eq. (6-15) e a expressão para a matriz $\mathcal{R}_{1 \mid N-1}$ definida na Eq.(6-18), pode-se estimas as matrizes de observabilidade e controlabilidade através das equações

$$
\begin{gathered}
\hat{\boldsymbol{\Gamma}}=\mathbf{U}_{n} \mathbf{S}_{n}^{1 / 2} \\
\hat{\mathcal{O}}=\mathbf{S}_{n}^{1 / 2} \mathbf{V}_{n}^{T} .
\end{gathered}
$$

Utilizando as estimativas acima e a matriz de Hankel $\mathcal{R}_{2 \mid N}$ definida na Eq. (6-15), a matriz A do modelo de espaço de estado pode ser estimada através da equação

$$
\hat{\mathbf{A}}=\hat{\boldsymbol{\Gamma}}^{\dagger} \mathcal{R}_{1 \mid N-1} \hat{\mathcal{O}}^{\dagger},
$$

onde $\hat{\boldsymbol{\Gamma}}^{\dagger}$ e $\hat{\mathcal{O}}^{\dagger}$ são as inversas generalizadas das matrizes $\hat{\boldsymbol{\Gamma}}$ e $\hat{\mathcal{O}}$, respectivamente. Uma vez estimada a matriz A e seus respectivos autovalores e autovetores, os parâmetros modais do sistema podem ser calculados através da Eq.(4-32). 
Como dito anteriormente, os sinais de um experimento contém sempre uma parcela de ruido, o que pode dificultar na escolha de quantos valores singulares precisam ser utilizados nas Eqs. (6-19/6-20). Por isso, é indicado que a identificação seja realizada diversas vezes para um número crescentes de valores singulares. Os respectivos resultados devem então ser marcados em um gráfico em função da possível ordem do sistema (número de valores singulares), construindo assim um diagrama de estabilização semelhante ao apresentado na seção anterior.

\section{Exemplo 6.2}

Para exemplificar o método ERA, a identificação dos parâmetros de um modelo de ponte suspensa foi feita. A estrutura testada é mostrada pela Figura 6.4. Neste exemplo, a banda de frequência analisada foi definida entre $0-100 \mathrm{~Hz}$. A frequência de aquisição foi escolhida como $1 \mathrm{kHz}$ para manter o fator de oversampling igual a 10. Para excitar a bancada, utilizou-se novamente o vento, que desta vez foi gerado por um ventilador de parede. Para medir as respectivas aceleração, oito acelerômetros triaxiais foram utilizados. Esses sensores foram posicionados ao longo eixo longitudinal da ponte de forma igualmente espaçados, como mostra a Figura 6.4. Dessa forma, apenas modos de flexão vertical e horizontal podem ser observados. Os modos de torção não foram analisados neste exemplo. Durante os testes, somente dois eixos dos acelerômetros foram utilizadas, totalizando assim 16 canais de medição.

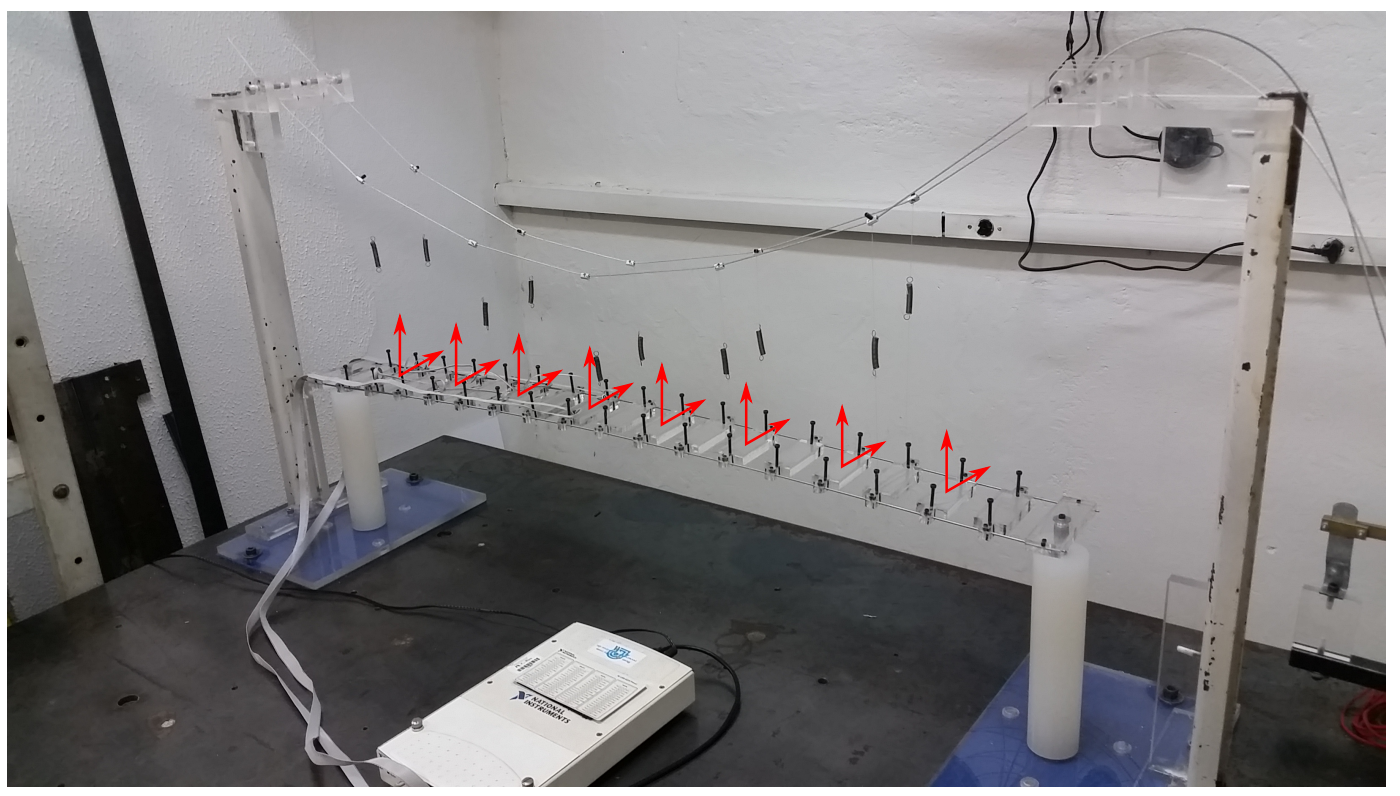

Figura 6.4: Bancada de uma ponte suspensa utilizada para ilustrar a identificação pelo método ERA

Durante o teste, foram medidas 204.800 amostras. O cálculo das correlações foi realizado para valores de $\tau_{k}$ variando entre 1 e 1025. Para o método 
ERA, as matrizes de Hankel foram construídas com $N$ igual a 10. Logo, o número máximo de blocos de colunas que se pôde usar foi $1014(1025-N-1)$. Como cada matriz de correlação tem dimensão $16 \times 16$ (duas direções em cada um dos oito acelerômetros), as dimensões reais das matrizes $\mathcal{R}_{1 \mid N}$ e $\mathcal{R}_{2 \mid N+1}$ são $160 \times 16224$. Por isso, ao realizar a SVD da matriz $\mathcal{R}_{1 \mid N}$ (Eq. (6-17)), a quantidade máxima de valores singulares que se pode obter é 160. Caso esse número não seja suficiente para construir um bom diagrama de estabilização, um número maior para $N$ deve ser escolhido.

Ao truncar a decomposição por valores singulares com $n$ (ordem do modelo de estado de espaço) na Eq. (6-18) variando entre 1 e 160, o diagrama de estabilização mostrado pela Figura 6.5 é obtido. Neste diagrama, inúmeras linhas de polos estáveis foram criadas. Para ter certeza que essas linhas estão relacionadas ao sistema, é preciso observar o formato dos respectivos modos.

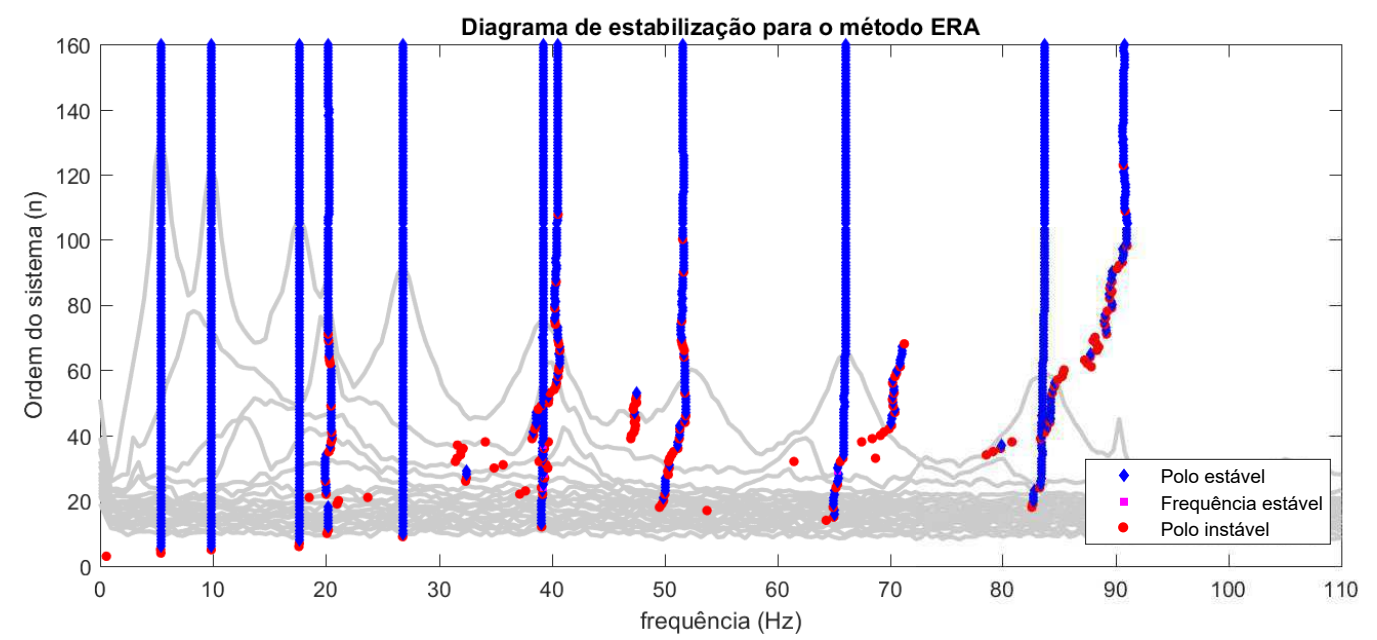

Figura 6.5: Diagrama de estabilização da bancada da ponte utilizando o método de identificação ERA

Para as onze linha estáveis no diagrama, os respectivos modos são mostradas na Figura 6.6. Como pode ser visto, o único modo que não foi bem identificado é o referente à frequência natural de valor $40.5 \mathrm{~Hz}$. Um dos motivos para essa identificação ter sido ruim é o fato de haver dois modos próximos um do outro $(39.25$ e $40.5 \mathrm{~Hz})$. Isso dificulta bastante o processo de identificação, pois inviabiliza a hipótese de coordenadas modais não correlacionadas feita no capítulo 3. A identificação só seria então possível caso o forçamento fosse mais semelhante a um ruído branco.

Para todos os outro modos, foi possivel identificar claramente os primeiros oito modos de flexão verticais, e dois modos de flexão horizontais (primeiro e possivelmente o quinto). Nota-se que alguns modos de flexão horizontal estão faltando. A principal explicação para isso é o fato do vento não ter conseguido 
excitar esses modos, uma vez que não há picos nos espectros da Figura 6.5 para esses modos.
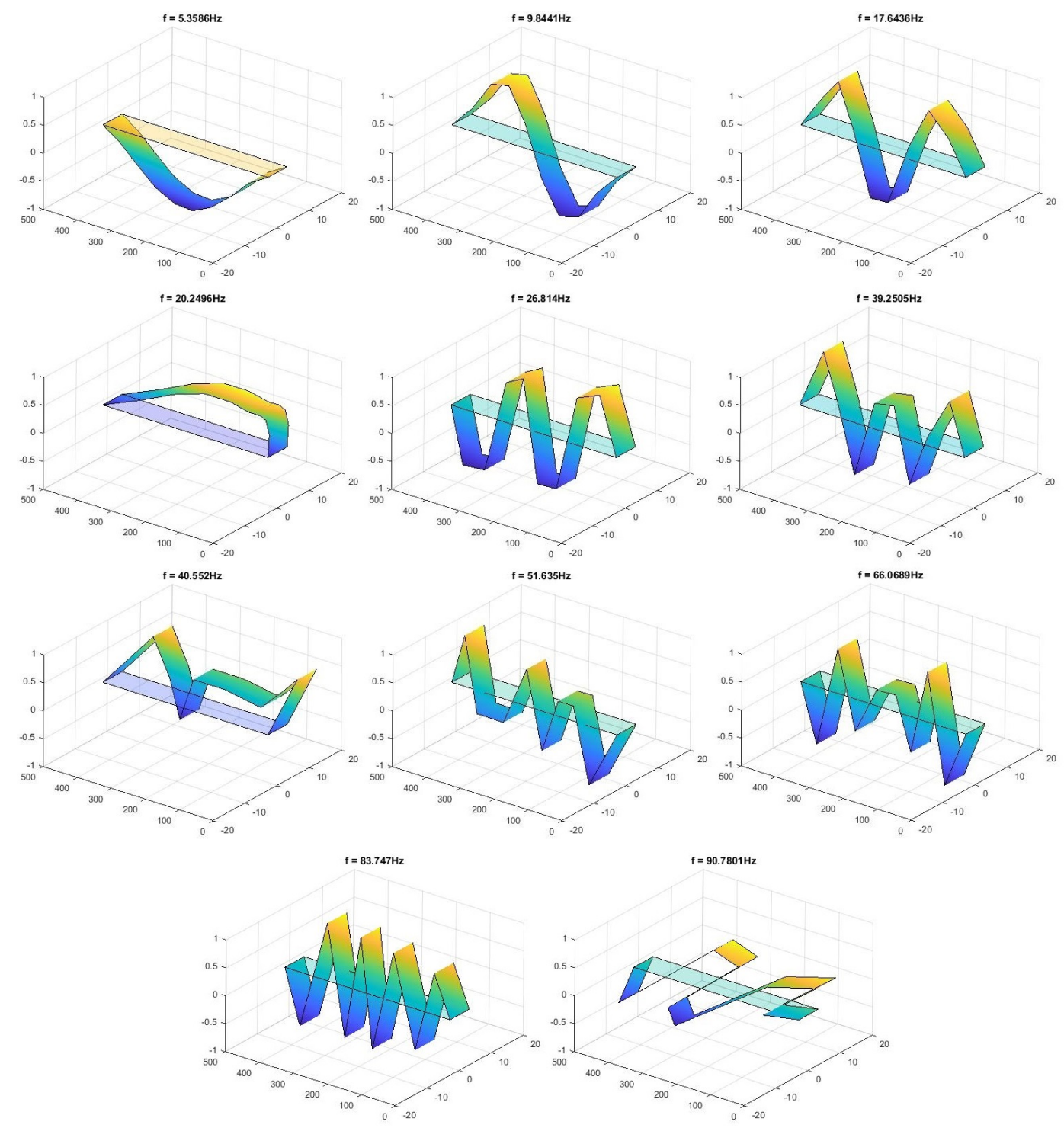

Figura 6.6: Formato dos modos de vibração da bancada da ponte identificados pelo método ERA

Os valores das frequências naturais e dos fatores de amortecimento identificados são mostrados na Tabela 6.2, assim como as respectivas classificações dos modos. Os valores encontrados para os fatores de amortecimento são ligeiramente altos para o que se costuma obter em análise modal de estruturas. Por isso, alguns outros métodos de identificação deveriam ser utilizados para garantir que esses valores estejam corretos. Percebe-se que os modos de flexão horizontal possuem um valor mais alto para o amortecimento quando comparados com os de flexão vertical, o que pode indicar um erro na identificação. (Fim do exemplo) 
Tabela 6.2: Parâmetros modais identificados pelo método ERA

\begin{tabular}{cccc}
\hline Modo & $\begin{array}{c}\text { Frequência } \\
\text { Natural }[\mathbf{H z}]\end{array}$ & $\begin{array}{c}\text { Fator de } \\
\text { Amortecimento }[\%]\end{array}$ & $\begin{array}{c}\text { Classificação } \\
\text { do modo }\end{array}$ \\
\hline $1^{\mathrm{O}}$ & 5.358 & 2.91 & $1^{\mathrm{o}}$ flex. vertical \\
$2^{\mathrm{o}}$ & 9.844 & 2.07 & $2^{\mathrm{o}}$ flex. vertical \\
$3^{\mathrm{o}}$ & 17.644 & 2.82 & $3^{\mathrm{o}}$ flex. vertical \\
$4^{\mathrm{o}}$ & 20.250 & 4.17 & $1^{\mathrm{O}}$ flex. horizontal \\
$5^{\mathrm{o}}$ & 26.814 & 2.90 & $4^{\mathrm{o}}$ flex. vertical \\
$6^{\mathrm{o}}$ & 39.251 & 3.56 & $5^{\mathrm{o}}$ flex. vertical \\
$7^{\mathrm{o}}$ & 40.552 & 9.74 & Indefinido \\
$8^{\mathrm{o}}$ & 51.635 & 7.88 & $6^{\mathrm{o}}$ flex. vertical \\
$9^{\mathrm{o}}$ & 66.069 & 3.26 & $7^{\mathrm{o}}$ flex. vertical \\
$10^{\mathrm{o}}$ & 83.747 & 3.26 & $8^{\mathrm{o}}$ flex.vertical \\
$11^{\mathrm{o}}$ & 90.780 & 11.49 & $4^{\mathrm{o}}$ ou $5^{\mathrm{o}}$ flex. horizontal \\
\hline
\end{tabular}

\section{3}

\section{Subespaço estocástico}

Entre todos os métodos de identificação no domínio do tempo, o método de subespaço estocástico (SSI) é sem dúvida o mais utilizado em análise modal operacional. No entanto, este método é também o mais complexo e de certa forma o mais abstrato. Na literatura, existem diversos algorítimos que realizam a identificação por subespaço estocástico. Nessa dissertação apenas os dois mais populares serão apresentados: o covariance-driven e o data-driven. Um estudo completo sobre esses algorítimos é encontrada em [38]. Atualmente, as pesquisas sobre SSI têm focado na quantificação de incertezas dos parâmetros modais identificados $[49,50]$.

Todos os algorítimos de SSI utilizam o modelo de espaço de estado definido pelas Eqs. (4-17/4-18), e repetidos a seguir por conveniência

$$
\begin{aligned}
\mathbf{x}(k+1) & =\mathbf{A} \mathbf{x}(k)+\mathbf{w}(k) \\
\mathbf{y}(k) & =\mathbf{C x}(k)+\mathbf{v}(k)
\end{aligned}
$$

Para uma compreensão mais fácil do método, o modelo apresentado acima precisa ser estendido para um novo formato. Para isso, as variáveis do modelo precisam ser rearrumadas em matrizes de Hankel. Uma escolha apropriada para as dimensões dessas matrizes é fundamental para o bom desempenho do método e serão discutidos mais a frente. A seguir, oito matrizes de Hankel são definidas utilizando as quatro variáveis do modelo $(\mathbf{x}(k), \mathbf{y}(k), \mathbf{w}(k), \mathbf{v}(k))$ : 


$$
\begin{aligned}
& \mathcal{Y}_{p}=\frac{1}{\sqrt{N}} \mathcal{Y}_{0 \mid q-1}=\left[\begin{array}{cccc}
\mathbf{y}(0) & \mathbf{y}(1) & \cdots & \mathbf{y}(N-1) \\
\mathbf{y}(1) & \mathbf{y}(2) & \ldots & \mathbf{y}(N) \\
\vdots & \vdots & \ddots & \vdots \\
\mathbf{y}(q-1) & \mathbf{y}(q) & \cdots & \mathbf{y}(q+N-2)
\end{array}\right] \\
& \mathcal{Y}_{f}=\frac{1}{\sqrt{N}} \mathcal{Y}_{q \mid 2 q-1}=\left[\begin{array}{cccc}
\mathbf{y}(q) & \mathbf{y}(q+1) & \ldots & \mathbf{y}(q+N-1) \\
\mathbf{y}(q+1) & \mathbf{y}(q+2) & \ldots & \mathbf{y}(q+N) \\
\vdots & \vdots & \ddots & \vdots \\
\mathbf{y}(2 q-1) & \mathbf{y}(2 q) & \ldots & \mathbf{y}(2 q+N-2)
\end{array}\right] \\
& \mathcal{W}_{p}=\frac{1}{\sqrt{N}} \mathcal{W}_{0 \mid q-1}=\left[\begin{array}{cccc}
\mathbf{w}(0) & \mathbf{w}(1) & \ldots & \mathbf{w}(N-1) \\
\mathbf{w}(1) & \mathbf{w}(2) & \ldots & \mathbf{w}(N) \\
\vdots & \vdots & \ddots & \vdots \\
\mathbf{w}(q-1) & \mathbf{w}(q) & \ldots & \mathbf{w}(q+N-2)
\end{array}\right] \\
& \mathcal{W}_{f}=\frac{1}{\sqrt{N}} \mathcal{W}_{q \mid 2 q-1}=\left[\begin{array}{cccc}
\mathbf{w}(q) & \mathbf{w}(q+1) & \ldots & \mathbf{w}(q+N-1) \\
\mathbf{w}(q+1) & \mathbf{w}(q+2) & \ldots & \mathbf{w}(q+N) \\
\vdots & \vdots & \ddots & \vdots \\
\mathbf{w}(2 q-1) & \mathbf{w}(2 q) & \ldots & \mathbf{w}(2 q+N-2)
\end{array}\right] \\
& \mathcal{V}_{p}=\frac{1}{\sqrt{N}} \mathcal{V}_{0 \mid q-1}=\left[\begin{array}{cccc}
\mathbf{v}(0) & \mathbf{v}(1) & \ldots & \mathbf{v}(N-1) \\
\mathbf{v}(1) & \mathbf{v}(2) & \ldots & \mathbf{v}(N) \\
\vdots & \vdots & \ddots & \vdots \\
\mathbf{v}(q-1) & \mathbf{v}(q) & \ldots & \mathbf{v}(q+N-2)
\end{array}\right] \\
& \mathcal{V}_{f}=\frac{1}{\sqrt{N}} \mathcal{V}_{q \mid 2 q-1}=\left[\begin{array}{cccc}
\mathbf{v}(q) & \mathbf{v}(q+1) & \ldots & \mathbf{v}(q+N-1) \\
\mathbf{v}(q+1) & \mathbf{v}(q+2) & \ldots & \mathbf{v}(q+N) \\
\vdots & \vdots & \ddots & \vdots \\
\mathbf{v}(2 q-1) & \mathbf{v}(2 q) & \ldots & \mathbf{v}(2 q+N-2)
\end{array}\right] \\
& \mathcal{X}_{p}=\frac{1}{\sqrt{N}} \mathcal{X}_{0 \mid 0}=\left[\begin{array}{llll}
\mathbf{x}(0) & \mathbf{x}(1) & \ldots & \mathbf{x}(N-1)
\end{array}\right] \\
& \mathcal{X}_{f}=\frac{1}{\sqrt{N}} \mathcal{X}_{q \mid q}=\left[\begin{array}{llll}
\mathbf{x}(q) & \mathbf{x}(q+1) & \ldots & \mathbf{x}(q+N-1)
\end{array}\right]
\end{aligned}
$$

Pode-se notar que cada uma das variáveis formam duas matrize, uma chamada de passado (com subscrito $\square_{p}$ ) e outra chamada de futuro (com subscrito $\square_{f}$ ). Para as matrizes $\mathcal{Y}_{p}, \mathcal{Y}_{f}, \mathcal{W}_{p}, \mathcal{W}_{f}, \mathcal{V}_{p}$ e $\mathcal{V}_{f}$, o que diferencia 
a noção de passado e futuro é o instante de tempo $q$ (lembrando que cada elemento destas matrizes são vetores). Utilizando essas oito matrizes de Hankel, o modelo de espaço de estado apresentado pela Eq. (6-22) pode ser reescrito na forma

$$
\begin{aligned}
\mathcal{X}_{f} & =\mathbf{A}^{q} \mathcal{X}_{p}+\boldsymbol{\Lambda} \mathcal{W}_{p} \\
\mathcal{Y}_{p} & =\boldsymbol{\Gamma} \mathcal{X}_{p}+\mathbf{H} \mathcal{W}_{p}+\mathcal{V}_{p} \\
\mathcal{Y}_{f} & =\boldsymbol{\Gamma} \mathcal{X}_{f}+\mathbf{H} \mathcal{W}_{f}+\mathcal{V}_{f}
\end{aligned}
$$

onde,

$$
\begin{gathered}
\boldsymbol{\Gamma}=\left[\begin{array}{c}
\mathbf{C} \\
\mathbf{C A} \\
\mathbf{C A}^{2} \\
\vdots \\
\mathbf{C A}^{q-1}
\end{array}\right] ; \quad \mathbf{H}=\left[\begin{array}{cccccc}
0 & 0 & 0 & \ldots & 0 \\
\mathbf{C} & 0 & 0 & \ldots & 0 \\
\mathbf{C A} & \mathbf{C} & 0 & \ldots & 0 \\
\vdots & \vdots & \vdots & \ddots & \vdots \\
\mathbf{C A}^{q-2} & \mathbf{C A}^{q-3} & \mathbf{C A}^{q-4} & \ldots & 0
\end{array}\right] \\
\Lambda=\left[\begin{array}{lllll}
\mathbf{A}^{q-1} & \mathbf{A}^{q-2} & \ldots & \mathbf{A} & \mathbf{I}
\end{array}\right] .
\end{gathered}
$$

Na equação (6-32), a matriz $\boldsymbol{\Gamma}$ é mais uma vez a matriz de observabilidade. Nos métodos de subespaço estocástico, o principal objetivo consiste em estimar $\Gamma$ para que depois as matrizes A e $\mathbf{C}$ possam ser extraídas. Para mostrar como isso pode ser feito utilizando apenas o sinal de resposta $\mathbf{y}(k)$, os algorítimos de cavariance-driven e data-driven serão apresentados a seguir divididos em três etapas [50,51]. A diferença entre esses dois algorítimos ocorre apenas durante a segunda etapa.

- Primeira etapa: Montagem das matrizes $\mathcal{Y}_{p}$ e $\mathcal{Y}_{f}$

Como em OMA as respostas do sistema são as únicas informações coletadas durante os experimentos, o método de subespaço estocástico inicia com a construção das matrizes de Hankel $\mathcal{Y}_{p}$ e $\mathcal{Y}_{f}$. Para isso, é necessário que o analista escolha corretamente a quantidade de blocos de linhas $q$ e o número de colunas $N$. Um procedimento para a escolha desses parâmetros será discutido mais a frente.

\section{- Segunda etapa: cálculo da matriz $\mathcal{H}$}

Essa etapa consiste em calcular uma matriz específica, aqui chamada de $\mathcal{H}$, que contém uma importante propriedade de fatoração. Os algorítimos de covariance-driven e data-driven se diferenciam apenas na forma com que essa matriz é calculada. A principal ideia é construir a matriz $\mathcal{H} \in \mathbb{R}^{q n_{o} \times q n_{o}}$ de forma que

$$
\mathcal{H}=\Gamma \mathrm{Z}
$$


onde $\mathbf{Z}$ é uma matriz constante que dependerá do algorítimo escolhido. Através dessa fatoração, a matriz de observabilidade pode ser estimada como sendo uma base para do espaço coluna de $\mathcal{H}$.

O algorítimo de covariance-driven calcula a matriz $\mathcal{H}$ através da equação

$$
\mathcal{H}_{\text {cov }}=\mathcal{Y}_{f} \mathcal{Y}_{p}^{T}
$$

Uma importante obervação é o fato do produto entre as matrizes de Hankel resultar em estimativas para as matrizes de correlação arrumadas em uma matriz de bloco Toeplitz [38, 50], isto é

$$
\mathcal{Y}_{f} \mathcal{Y}_{p}^{T}=\left[\begin{array}{cccc}
\mathbf{R}_{y y}(q) & \mathbf{R}_{y y}(q-1) & \ldots & \mathbf{R}_{y y}(1) \\
\mathbf{R}_{y y}(q+1) & \mathbf{R}_{y y}(q) & \ldots & \mathbf{R}_{y y}(2) \\
\vdots & \vdots & \ddots & \vdots \\
\mathbf{R}_{y y}(2 q-1) & \mathbf{R}_{y y}(2 q-2) & \ldots & \mathbf{R}_{y y}(q)
\end{array}\right]
$$

Utilizando a extensão do modelo de espaço de estado definida pela Eq. (6-31), a definição de $\mathcal{H}_{\text {cov }}$ pode ser reescrita na forma

$$
\mathcal{H}_{\text {cov }}=\left(\boldsymbol{\Gamma} \mathcal{X}_{f}+\mathbf{H} \mathcal{W}_{f}+\mathcal{V}_{f}\right) \mathcal{Y}_{p}^{T}
$$

Supondo que as respostas do sistema não sejam correlacionadas com os futuros forçamentos e com os ruídos dos sensores, pode-se impor que $\mathcal{W}_{f} \mathcal{Y}_{p}^{T}=\mathcal{V}_{f} \mathcal{Y}_{p}^{T}=0$. Assim, a Eq. (6-36) se resume a

$$
\mathcal{H}_{\text {cov }}=\Gamma \mathcal{X}_{f} \mathcal{Y}_{p}^{T}=\Gamma \mathbf{Z}_{\text {cov }}
$$

Já no algorítimo data-driven, o cálculo de $\mathcal{H}$ é feito através de projeções ortogonais do espaço linha da matriz $\mathcal{Y}_{f}$ no espaço coluna da matriz $\mathcal{Y}_{p}$. Isso pode ser realizado através da equação

$$
\mathcal{H}_{\text {dat }}=\operatorname{Proj}_{\mathcal{Y}_{p}}\left(\mathcal{Y}_{f}\right)=\mathcal{Y}_{f} \mathcal{Y}_{p}^{T}\left(\mathcal{Y}_{p} \mathcal{Y}_{p}^{T}\right)^{\dagger} \mathcal{Y}_{p}
$$

Utilizando a Eq. (6-34), pode-se reescrever essa projeção como sendo

$$
\begin{aligned}
\mathcal{H}_{\text {dat }} & =\mathcal{H}_{\text {cov }}\left(\mathcal{Y}_{p} \mathcal{Y}_{p}^{T}\right)^{\dagger} \mathcal{Y}_{p} \\
& =\Gamma \mathbf{Z}_{\text {cov }}\left(\mathcal{Y}_{p} \mathcal{Y}_{p}^{T}\right)^{\dagger} \mathcal{Y}_{p} \\
& =\Gamma \mathbf{Z}_{\text {dat }}
\end{aligned}
$$

Dessa forma, mostra-se que $\mathcal{H}_{d a t}$ também possui a fatoração exigida.

\section{- Terceira etapa: identificação dos parâmetros modais}

Independente do algorítimo escolhido, Overschee e De Moor [38] provam que $\mathcal{H}$ e $\boldsymbol{\Gamma}$ possuem o mesmo espaço coluna. Supondo que o sistema 
seja completamente observável, é esperado que a matriz $\Gamma$ tenha posto completo e seja igual a $n$, ordem do modelo de espaço de estado. Por isso, após uma decomposição por valores singulares de $\mathcal{H}$, apenas $n$ valores singulares não-zeros deveriam ser obtidos. Como em um caso experimental os sinais têm sempre ruído, essa quantidade de valores singulares pode ser maior. Como visto nas duas seções anteriores, a melhor forma de escolher a verdadeira ordem do sistema é através dos diagramas de estabilização.

Supondo que $\mathcal{H}$ tenha sido obtida corretamente, sua decomposição por valores singulares pode ser escrita na forma reduzida através da equação

$$
\mathcal{H}=\left[\begin{array}{ll}
\mathbf{U}_{1} & \mathbf{U}_{2}
\end{array}\right]\left[\begin{array}{cc}
\mathbf{S}_{1} & 0 \\
0 & 0
\end{array}\right]\left[\begin{array}{c}
\mathbf{V}_{1}^{T} \\
\mathbf{V}_{2}^{T}
\end{array}\right]=\mathbf{U}_{1} \mathbf{S}_{1} \mathbf{V}_{1}^{T}
$$

Uma estimativa para a matriz de observabilidade pode então ser obtida por

$$
\hat{\Gamma}=\mathbf{U}_{1} \mathbf{S}_{1}^{1 / 2}
$$

Utilizando a definição da matriz $\Gamma$ na Eq. (6-32), a matriz de saída $\mathbf{C}$ pode ser identificada de forma imediata como sendo as primeiras $n_{o}$ (quantidade de repostas medidas) linhas de $\hat{\boldsymbol{\Gamma}}$. A matriz $\mathbf{A}$ pode ser estimada utilizando um método de mínimos quadrados. Definindo $\bar{\Gamma}$ e $\underline{\boldsymbol{\Gamma}}$ como sendo a matriz $\hat{\boldsymbol{\Gamma}}$ sem as primeiras e últimas $n_{o}$ linhas, respectivamente, têm-se

$$
\overline{\boldsymbol{\Gamma}}=\left[\begin{array}{c}
\mathrm{CA} \\
\vdots \\
\mathrm{CA}^{q-1}
\end{array}\right] ; \quad \underline{\boldsymbol{\Gamma}}=\left[\begin{array}{c}
\mathrm{C} \\
\vdots \\
\mathrm{CA}^{q-2}
\end{array}\right]
$$

A matriz A pode então ser estimada através da equação

$$
\hat{\mathbf{A}}=\underline{\Gamma}^{\dagger} \overline{\boldsymbol{\Gamma}} .
$$

Uma vez obtida as matrizes $\hat{\mathbf{C}}$ e $\hat{\mathbf{A}}$, os parâmetros modais do sistema podem ser identificados utilizando a Eq. (4-32).

Durante a primeira etapa do método, as matrizes $\mathcal{Y}_{p}$ e $\mathcal{Y}_{f}$ precisam ser corretamente construídas. Uma escolha apropriada para as dimensões dessas matrizes é essencial para o bom funcionamento do método. A seguir, um procedimento de como selecionar a quantidade de blocos de linhas $q$ e a quantidade de colunas $N$ é apresentado.

Durante a segunda etapa, foi mostrado como a matriz $\mathcal{H}$ é obtida e que sua respectiva dimensão é $q n_{o} \times q n_{o}$. Por isso, quando realizado sua SVD na Eq. (6-40), a quantidade máxima de valores singulares que se pode obter é $q n_{o}$. Para que todos os modos do sistema sejam obtidos, é necessário que $q$ 
seja pelo menos maior que $n / n_{o}$. No entanto, como em um caso real a ordem $n$ não é conhecida, é recomendado que $q$ seja grande o suficiente até que valores singulares próximos a zero sejam obtidos. Isso indica que todos os modos do sistema já forma obtidos e que aumentar ainda mais a quantidade de polos não traz novas informações. Esse procedimento é ilustrado pelo diagrama de estabilização.

Para determinar se o número de colunas utilizadas no método foi satisfatório, a decomposição por valores singulares de $\mathcal{H}$ deve ser realizada diversas vezes. Durante a segunda etapa, mostrou-se que $\mathcal{H}$ foi obtida através de produtos entre as matrizes $\mathcal{Y}_{p}$ e $\mathcal{Y}_{f}$. Esses produtos resultam em estimativas para as matrizes de correlação utilizando $N$ amostras. Os valores reais dessas correlações só são obtidas quando $N \rightarrow \infty$, o que significa que $N$ precisa ser grande o suficiente até que essas matrizes de correlação, e consequentemente os valores singulares de $\mathcal{H}$, convirjam para o valor correto. Um procedimento para definir se $N$ é suficientemente grande consiste em calcular os valores singulares de $\mathcal{H}$ repetidamente com valores crescentes de $N$ até que eles se estabilizem. Esse procedimento é ilustrado pela Figura 6.9. Neste caso, $N=5 \times 10^{4}$ é suficiente para estabilizar os valores singulares.

\section{Exemplo 6.3}

Nesta seção, utilizou-se uma pequena pá de turbina eólica para exemplificar o método de identificação por subespaço estocástico. Neste exemplo, apenas o algorítimo de covariance-driven será ilustrado. A estrutura testada foi engastada em um dos lados e mantida livre do outro, como mostra a Figura 6.7. A fonte de excitação escolhida foi novamente o vento gerado pelo soprador apresentado no Exemplo 6.1.

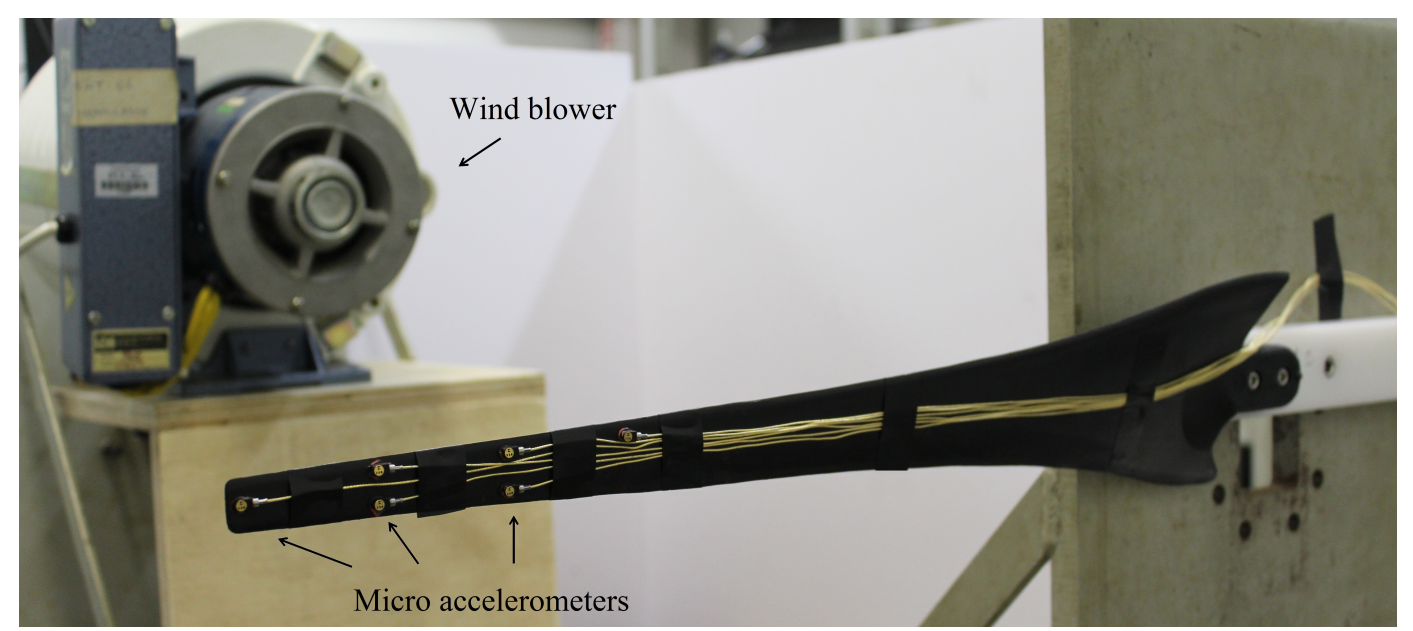

Figura 6.7: Pá eólica utilizada para ilustrar o método de identificação por subespaço estocástico 
As resposta do sistema foram medidas por seis micro acelerômetros piezoelétricos em dezenove diferentes posições. Como somente seis acelerômetros estavam disponiveis, quatro diferentes testes foram realizados individualmente como mostrado pela Figura 6.8. Um sensor de referência, marcado na figura por uma estrela, foi mantido na mesma posição durante todos os quatro teste para poder definir todos os modos com a mesma escala. A localização deste acelerômetro foi definida considerando as possíveis amplitudes de todos os modos, inclusive os de torção. Uma importante característica desta localização é o fato dela não poder ser um ponto nodal em nenhum dos modos da estrutura.

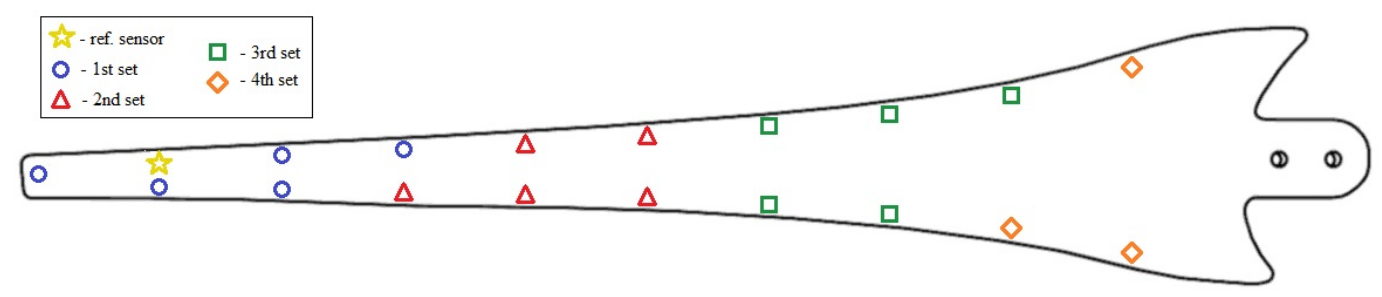

Figura 6.8: Posicionamento dos acelerômetros durante os quatro teste

Neste exemplo, a frequência de interesse foi definida como sendo entre $0-500 \mathrm{~Hz}$. Para obter um fator 10 de oversampling, a frequência de aquisição foi então escolhida com sendo igual a $5 \mathrm{kHz}$. Em cada um dos teste, 409.600 amostras de cada um dos sensores foram coletadas. Definiu-se a quantidade de blocos de linha $q$ para as matrizes de Hankel igual a 30. Como $n_{o}=6$, a máxima ordem que se pôde identificar para o sistema foi 180. Em relação ao número de colunas, utilizou-se todas as amostras disponíveis, fazendo com que $N=409.541$. Esse valor é suficiente ao analisar o diagrama de estabilização dos valores singulares de $\mathcal{H}$.

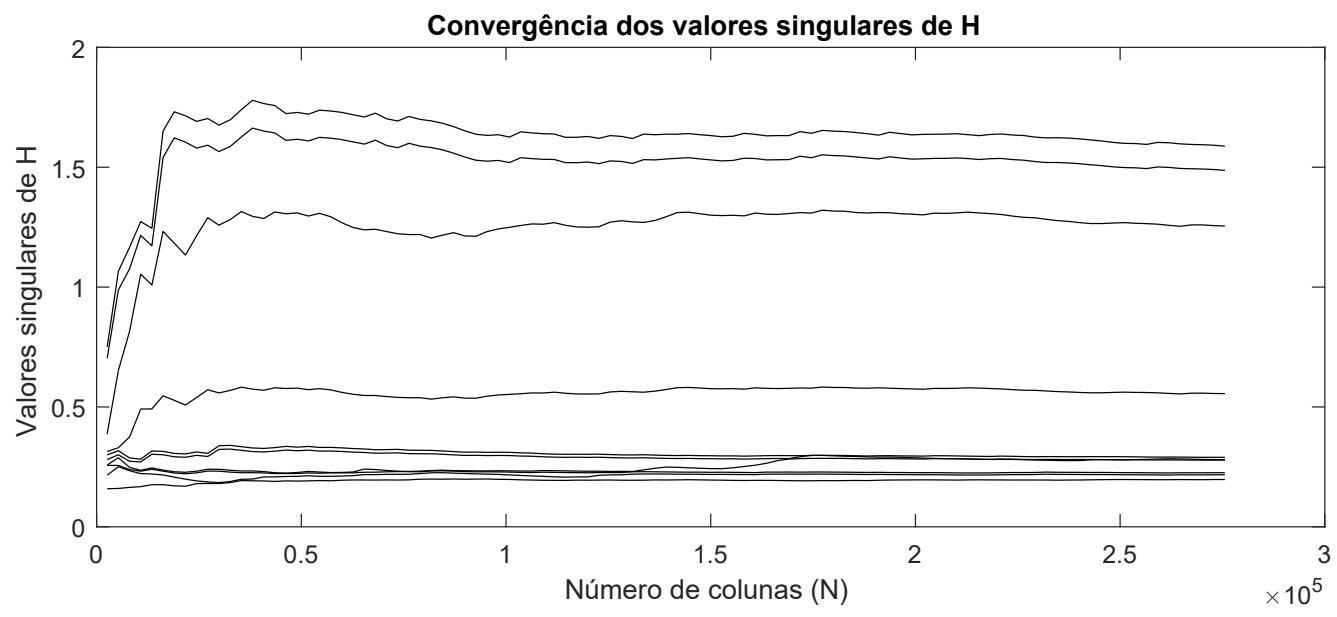

Figura 6.9: Gráfico de convergência dos valores singulares de $\mathcal{H}$ utilizando diferentes números de colunas em $\mathcal{Y}_{p}$ e $\mathcal{Y}_{f}$ 
Um diagrama de estabilização para os parâmetros modais foi construído para cada um dos testes realizados na estrutura, variando os valores de $n$ (ordem da identificação) entre 1 e 180. Os resultados para cada um dos testes são mostrados na Figura 6.10. Percebe-se que em todos os diagramas, aproximadamente seis linhas estáveis foram criadas. Em particular, a primeira linha não poderia ser considerada estável, pois existe muitos polos instáveis em sua composição. Mesmo assim, supõe-se que estes polos estão sim vinculados a um dos modos do sistema, uma vez que as respectivas frequências naturais estão alinhadas a um dos picos dos espectros. O principal motivo para a identificação deste modo não ter sido bem feita é o fato dos acelerômetros não funcionarem bem em frequências abaixo de $20 \mathrm{~Hz}$.

Os respectivos valores encontrados para as frequências naturais e fatores de amortecimento dos quatro testes são mostrados pelas tabelas 6.3-6. Os valores paras as frequências naturais foram próximas em todos os testes enquanto os de amortecimento não. O fator de amortecimento de uma estrutura é sempre um parâmetro mais difícil de ser estimado. Para este caso em particular, o motivo encontrado para a diferença entre os valores consiste nos diferentes posicionamentos dos cabos dos acelerômetros em cada um dos testes.

Tabela 6.3: Parâmetros modais identificados pelo método SSI - Teste 1

\begin{tabular}{cccc}
\hline Modo & $\begin{array}{c}\text { Frequência } \\
\text { Natural }[\mathbf{H z}]\end{array}$ & $\begin{array}{c}\text { Fator de } \\
\text { Amortecimento }[\%]\end{array}$ & $\begin{array}{c}\text { Classificação } \\
\text { do modo }\end{array}$ \\
\hline $1^{\mathrm{o}}$ & 18.95 & 0.88 & $1^{\mathrm{o}}$ flex. horizontal \\
$2^{\mathrm{o}}$ & 63.87 & 1.04 & $2^{\mathrm{o}}$ flex. horizontal \\
$3^{\mathrm{o}}$ & 146.47 & 1.57 & $3^{\mathrm{o}}$ flex. horizontal \\
$4^{\mathrm{o}}$ & 250.30 & 1.51 & $4^{\mathrm{o}}$ flex. horizontal \\
$5^{\mathrm{o}}$ & 290.45 & 0.89 & $1^{\mathrm{o}}$ torção \\
$6^{\mathrm{o}}$ & 376.82 & 2.01 & $5^{\mathrm{o}}$ flex. horizontal \\
\hline
\end{tabular}

Tabela 6.4: Parâmetros modais identificados pelo método SSI - Teste 2

\begin{tabular}{cccc}
\hline Modo & $\begin{array}{c}\text { Frequência } \\
\text { Natural }[\mathbf{H z}]\end{array}$ & $\begin{array}{c}\text { Fator de } \\
\text { Amortecimento [\%] }\end{array}$ & $\begin{array}{c}\text { Classificação } \\
\text { do modo }\end{array}$ \\
\hline $1^{\mathrm{O}}$ & 18.94 & 1.07 & $1^{\mathrm{O}}$ flex. horizontal \\
$2^{\mathrm{O}}$ & 63.87 & 0.93 & $2^{\mathrm{O}}$ flex. horizontal \\
$3^{\mathrm{O}}$ & 145.68 & 2.23 & $3^{\mathrm{O}}$ flex. horizontal \\
$4^{\mathrm{O}}$ & 248.32 & 1.18 & $4^{\mathrm{O}}$ flex. horizontal \\
$5^{\mathrm{o}}$ & 283.46 & 1.01 & $1^{\mathrm{o}}$ torção \\
$6^{\mathrm{O}}$ & 375.70 & 1.58 & $5^{\mathrm{o}}$ flex. horizontal \\
\hline
\end{tabular}

Os respectivos modos associados a essas frequências naturais são mostrados pela Figura 6.11. Percebe-se que, assim como nos diagramas de estabilização, o primeiro modo não é bem identificado. Mesmo assim, consegue-se 

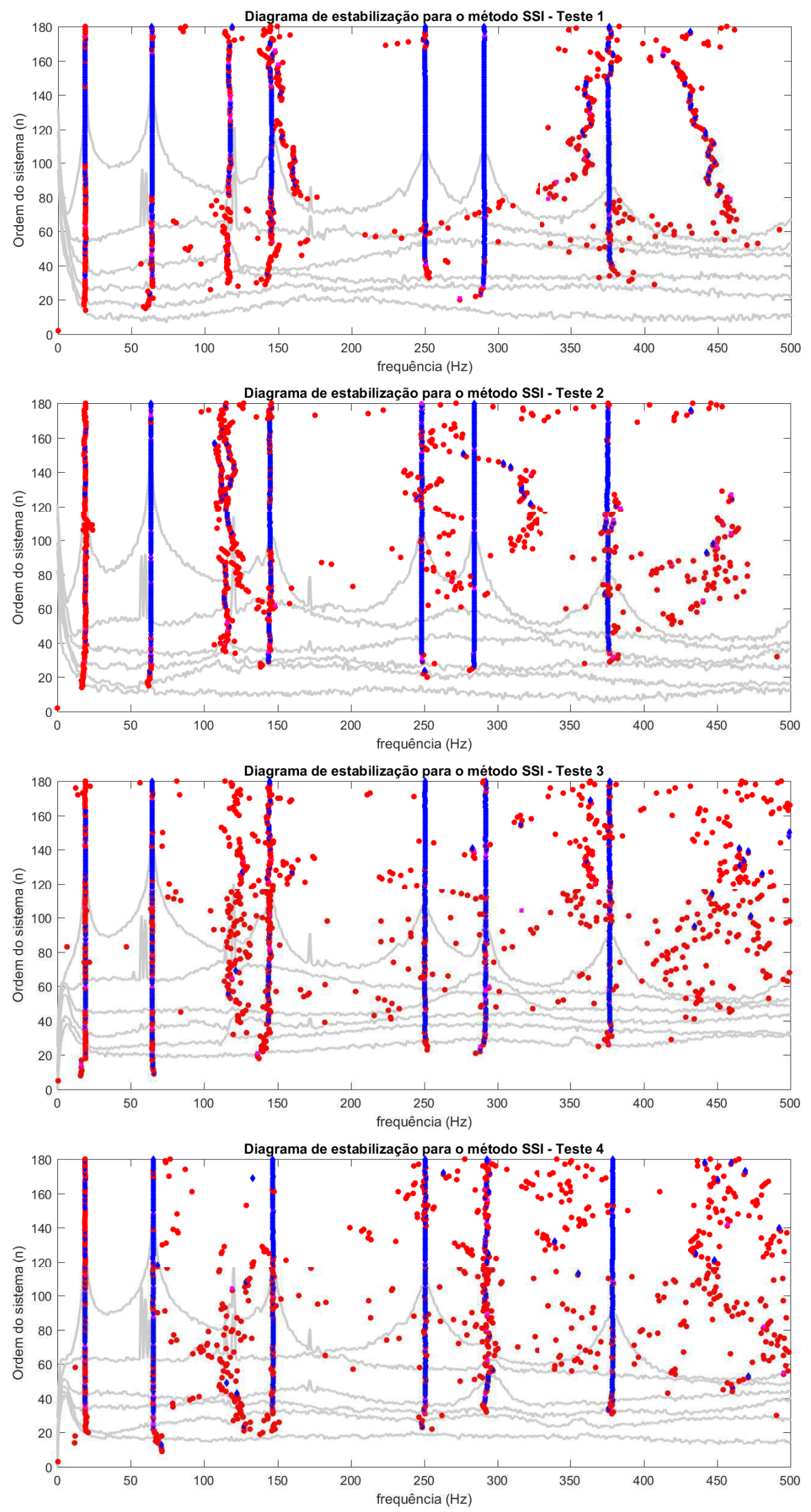

Figura 6.10: Diagramas de estabilização para os quatro testes feitos na pá eólica utilizando o método de identificação por subespaço estocástico 
Tabela 6.5: Parâmetros modais identificados pelo método SSI - Teste 3

\begin{tabular}{cccc}
\hline Modo & $\begin{array}{c}\text { Frequência } \\
\text { Natural }[\mathbf{H z}]\end{array}$ & $\begin{array}{c}\text { Fator de } \\
\text { Amortecimento [\%] }\end{array}$ & $\begin{array}{c}\text { Classificação } \\
\text { do modo }\end{array}$ \\
\hline $1^{\mathrm{O}}$ & 19.06 & 1.17 & $1^{\mathrm{O}}$ flex. horizontal \\
$2^{\mathrm{o}}$ & 64.55 & 0.84 & $2^{\mathrm{o}}$ flex. horizontal \\
$3^{\mathrm{O}}$ & 144.92 & 2.70 & $3^{\mathrm{o}}$ flex. horizontal \\
$4^{\mathrm{o}}$ & 250.40 & 1.80 & $4^{\mathrm{o}}$ flex. horizontal \\
$5^{\mathrm{o}}$ & 191.98 & 0.75 & $1^{\mathrm{o}}$ torção \\
$6^{\mathrm{o}}$ & 377.77 & 1.26 & $5^{\mathrm{o}}$ flex. horizontal \\
\hline
\end{tabular}

Tabela 6.6: Parâmetros modais identificados pelo método SSI - Teste 4

\begin{tabular}{cccc}
\hline Modo & $\begin{array}{c}\text { Frequência } \\
\text { Natural }[\mathbf{H z}]\end{array}$ & $\begin{array}{c}\text { Fator de } \\
\text { Amortecimento [\%] }\end{array}$ & $\begin{array}{c}\text { Classificação } \\
\text { do modo }\end{array}$ \\
\hline $1^{\mathrm{O}}$ & 19.08 & 1.26 & $1^{\mathrm{O}}$ flex. horizontal \\
$2^{\mathrm{o}}$ & 65.35 & 1.02 & $2^{\mathrm{o}}$ flex. horizontal \\
$3^{\mathrm{O}}$ & 146.92 & 1.35 & $3^{\mathrm{o}}$ flex. horizontal \\
$4^{\mathrm{o}}$ & 250.72 & 1.30 & $4^{\mathrm{o}}$ flex. horizontal \\
$5^{\mathrm{o}}$ & 294.91 & 0.99 & $1^{\mathrm{o}}$ torção \\
$6^{\mathrm{o}}$ & 378.52 & 1.04 & $5^{\mathrm{o}}$ flex. horizontal \\
\hline
\end{tabular}

defini-lo como sendo o primeiro modo de flexão horizontal da pá. Todos os outros modos foram bem identificados. No total, identificou-se os cinco primeiros modos de flexão horizontal e o primeiro modo de torção da pá. (Fim do exemplo) 

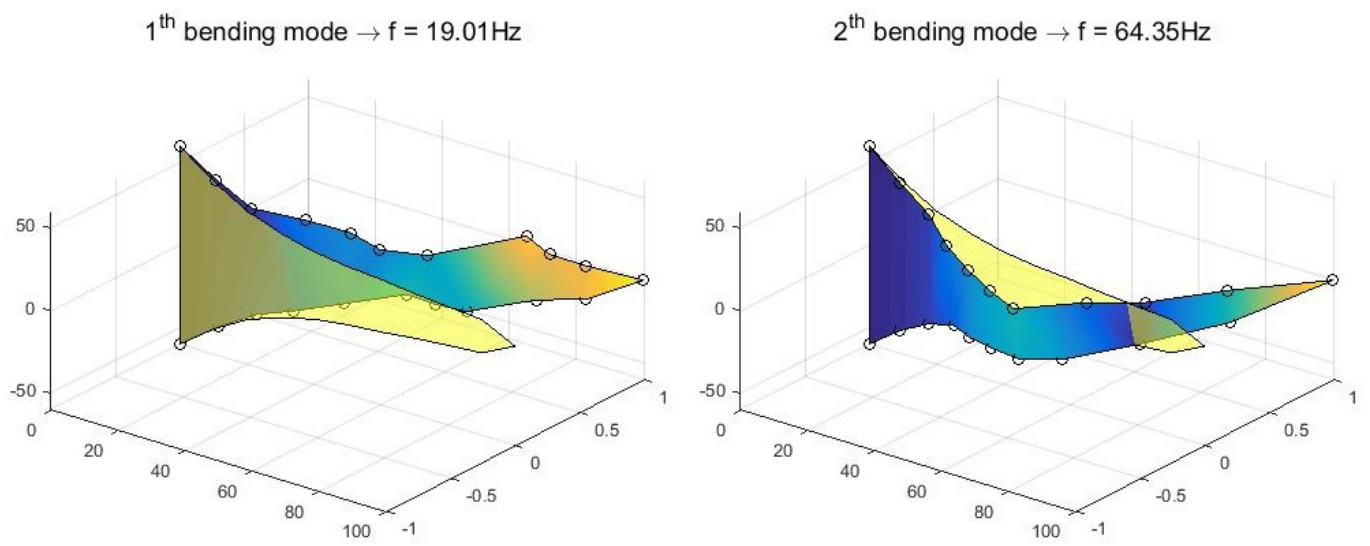

$3^{\text {th }}$ bending mode $\rightarrow \mathrm{f}=146 \mathrm{~Hz}$

$4^{\text {th }}$ bending mode $\rightarrow f=249.94 \mathrm{~Hz}$
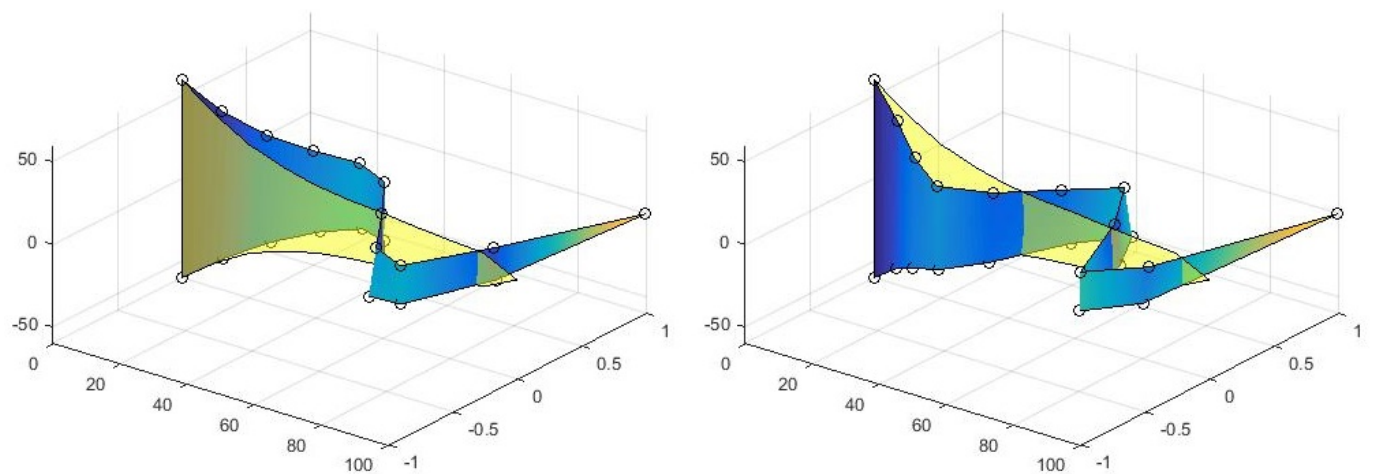

$1^{\text {st }}$ torsional mode $-\mathrm{f}=290.2 \mathrm{~Hz}$

$5^{\text {th }}$ bending mode $\rightarrow f=377.2 \mathrm{~Hz}$
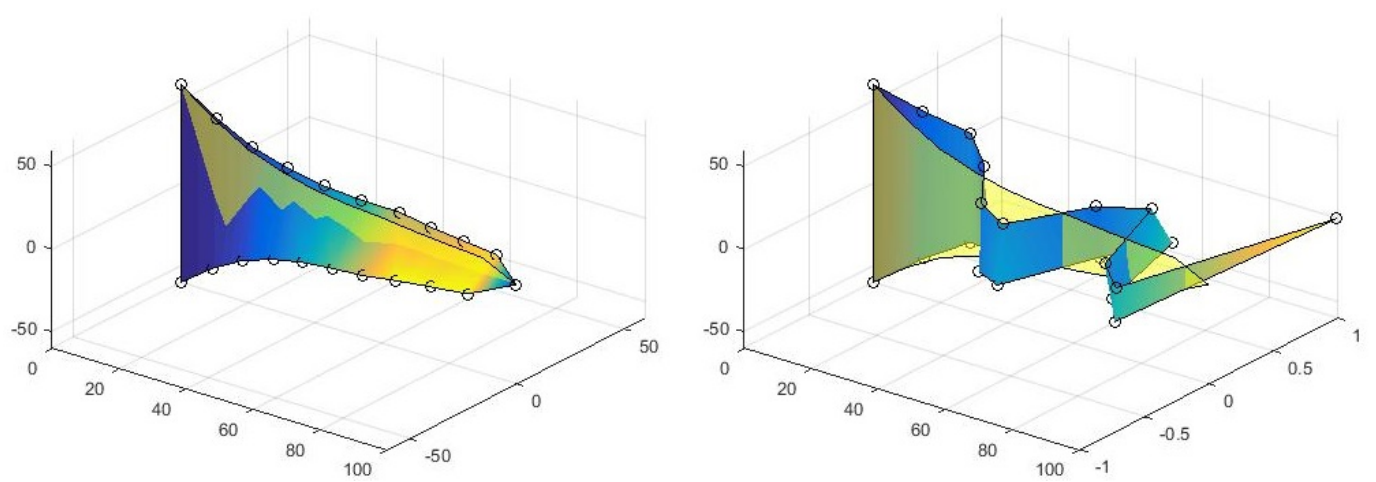

Figura 6.11: Formato dos modos de vibração da pá eólica identificados pelo método de subespaço estocástico 


\section{7 \\ Novos métodos de identificação no domínio do tempo}

Diferentemente do capítulo anterior, os próximos dois métodos foram desenvolvidos e aprimorados nesta dissertação. Por serem inéditos na literatura, esse capítulo representa a principal contribuição do ponto de vista de inovação. Em análise modal operacional, os métodos de decomposição ortogonal constituem uma nova família de técnicas de identificação. Todos eles foram desenvolvidos como extensões da Decomposição Ortogonal Própria (POD) $[52,53,54,55]$ para melhorar seu desempenho em análise modal .

As inovações apresentadas a seguir aparecem na forma com que os métodos são implementados. Elas consistem na adição de um filtro passa banda deslizante e na utilização de correlações com diferentes defasagens de tempo nos métodos de Smooth Orthogonal Decomposition (SOD) e State-Variable Modal Decomposition (SVMD). Através do filtro deslizante, elimina-se a relação entre a quantidade de modos identificados com o número de observações feita no sistema. Dessa forma pode-se identificar mais modos que o número de sensores utilizados durante os testes. Já a utilização de correlações com diferentes defasagens de tempo permite uma melhora da sensibilidade dos métodos em relação aso ruídos. Além disso, essa implementação permite que as incertezas dos parâmetros modais sejam quantificadas.

Embora as duas contribuições sejam aplicáveis nos dois métodos (SOD e SVMD), escolheu-se por apresentá-las de forma separadamente, isto é, apenas uma em cada um dos método. Por isso, o filtro deslizante só será apresentado junto ao método SOD, enquanto a utilização de correlações com diferentes defasagens de tempo só será apresentada no método SVMD. Para ilustrar os procedimentos de identificação, simulações numéricas são utilizadas.

\section{1}

\section{Smooth orthogonal decomposition}

O método de Smooth Orthogonal Decomposition, também conhecido como Smooth Karhunen-Loève Decomposition [56], é um método que adiciona uma restrição de suavidade na Decomposição Ortogonal Própria. Dessa forma, o SOD permite que, além dos modos normais, as frequências naturais de um sistema conservativo sejam identificadas [57, 58, 59, 60, 61, 62]. Ao ser 
comparado com os três métodos do capítulo anterior, o SOD é muito mais simples do ponto de vista computacional.

Neste método, a base para a decomposição é definida como sendo aquela que, ao ser usada na projeção de um campo escalar (deslocamento, velocidade e aceleração), obtém como resultado um outro campo com máxima variância e o mais suave possível em relação ao tempo. Esse método foi introduzido em análise modal por Chelidze e Zhou [63] em 2006 para o caso de vibração livre e por Farroq e Feeny [64] em 2008 para o caso de forçamento aleatório. Bellizzi e Sampaio [65] transformaram o SOD em uma ferramenta para análise modal de sistemas não lineares.

Seja $\mathbf{y}(t) \in \mathbb{R}^{n_{o}}$ o vetor de observações das respostas do sistema forçado por um sinal aleatório. Supõe-se que $\mathbf{y}(t)$ seja um processo estacionário com média zero. A ideia do SOD é encontrar vetores $\gamma_{i} \in \mathbb{R}^{n_{o}}, i=1, \ldots, n_{o}$, que criam uma base para a projeção ortogonal do campo gerado por $\mathbf{y}(t)$, cujo resultado tenha a máxima variância e que seja o mais suave possível em relação ao tempo.

Seja $\mathbf{v}_{i}(t)$ a projeção ortogonal de $\mathbf{y}(t)$ em relação ao vetor $\gamma_{i}$ definida por

$$
\mathbf{v}_{i}(t)=\operatorname{proj}_{\gamma_{i}} \mathbf{y}(t)=\frac{\left\langle\mathbf{y}(t), \boldsymbol{\gamma}_{i}\right\rangle}{\left\|\boldsymbol{\gamma}_{i}\right\|} \gamma_{i}, \quad i=1, \ldots, n_{o},
$$

onde $\langle\square, \square\rangle$ é o produto interno e $\|\square\|$ é a norma euclidiana de um vetor. Supondo por simplicidade que todos os vetores $\gamma_{i}$ são ortonormais, a norma de $\mathbf{v}_{i}(t)$ passa a ser o produto interno $\left\langle\mathbf{y}(t), \boldsymbol{\gamma}_{i}\right\rangle$. A máxima variância desta norma é então definida pela equação

$$
\begin{aligned}
\max _{\gamma_{i}}\left\{\mathbf{E}\left[\left\|\mathbf{v}_{i}(t)\right\|^{2}\right]\right\} & =\max _{\gamma_{i}}\left\{\mathbf{E}\left[\left\langle\boldsymbol{\gamma}_{i}, \mathbf{y}(t)\right\rangle \cdot\left\langle\mathbf{y}(t), \boldsymbol{\gamma}_{i}\right\rangle\right]\right\} \\
& =\max _{\gamma_{i}}\left\{\boldsymbol{\gamma}_{i}^{T} \mathbf{E}\left[\mathbf{y}(t) \mathbf{y}^{T}(t)\right] \boldsymbol{\gamma}_{i}\right\}
\end{aligned}
$$

$\mathrm{Na}$ equação acima, utilizou-se a suposição de média zero para $\mathbf{y}(t)$ e a propriedade comutativa do produto interno.

De acordo com a definição do SOD, o campo resultante aós a projeção também precisa ser o mais suave possível em relação a $t$. Para alcançar esse requisito, a variância da norma da derivada de $\mathbf{v}_{i}(t)$ precisa ser minimizada, resultando na equação

$$
\begin{aligned}
\min _{\gamma_{i}}\left\{\mathbf{E}\left[\left\|\dot{\mathbf{v}}_{i}(t)\right\|^{2}\right]\right\} & =\min _{\gamma_{i}}\left\{\mathbf{E}\left[\left\langle\gamma_{i}, \dot{\mathbf{y}}(t)\right\rangle \cdot\left\langle\dot{\mathbf{y}}(t), \gamma_{i}\right\rangle\right]\right\} \\
& =\min _{\gamma_{i}}\left\{\boldsymbol{\gamma}_{i}^{T} \mathbf{E}\left[\dot{\mathbf{y}}(t) \dot{\mathbf{y}}^{T}(t)\right] \boldsymbol{\gamma}_{i}\right\}
\end{aligned}
$$

Os problemas de maximização e de minimação acima podem ser transformados em apenas um problema de maximização ao definir a função $\alpha\left(\boldsymbol{\gamma}_{i}\right)$ 
na forma

$$
\max _{\gamma_{i}}\left\{\alpha\left(\boldsymbol{\gamma}_{i}\right)=\frac{\mathbf{E}\left[\left\|\mathbf{v}_{i}(t)\right\|^{2}\right]}{\mathbf{E}\left[\left\|\dot{\mathbf{v}}_{i}(t)\right\|^{2}\right]}\right\} \Rightarrow \max _{\gamma_{i}}\left\{\frac{\boldsymbol{\gamma}_{i}^{T} \mathbf{E}\left[\mathbf{y}(t) \mathbf{y}^{T}(t)\right] \boldsymbol{\gamma}_{i}}{\boldsymbol{\gamma}_{i}^{T} \mathbf{E}\left[\dot{\mathbf{y}}(t) \dot{\mathbf{y}}^{T}(t)\right] \boldsymbol{\gamma}_{i}}\right\} .
$$

Utilizando a definição das matrizes de correlação apresentada pela Eq. (3-2), esse problema de maximização pode ser reescrito na forma

$$
\max _{\gamma_{i}}\left\{\frac{\boldsymbol{\gamma}_{i}^{T} \mathbf{R}_{y y}(0) \boldsymbol{\gamma}_{i}}{\boldsymbol{\gamma}_{i}^{T} \mathbf{R}_{\dot{y} \dot{y}}(0) \boldsymbol{\gamma}_{i}}\right\}
$$

onde $\mathbf{R}_{y y}(0)$ e $\mathbf{R}_{\dot{y} \dot{y}}(0)$ são as matrizes de correlação das respostas e das sua derivadas, respectivamente, sem defasagem de tempo entre os sinais $(\tau=0)$.

A solução para esse problema de é obtida encontrando o seu ponto de estacionalidade, que é dado pela equação

$$
\nabla \alpha\left(\boldsymbol{\gamma}_{i}\right)=0=\frac{2\left[\boldsymbol{\gamma}_{i}^{T} \mathbf{R}_{\dot{y} \dot{y}}(0) \boldsymbol{\gamma}_{i}\right] \mathbf{R}_{y y}(0) \boldsymbol{\gamma}_{i}-2\left[\boldsymbol{\gamma}_{i}^{T} \mathbf{R}_{y y}(0) \boldsymbol{\gamma}_{i}\right] \mathbf{R}_{\dot{y} \dot{y}}(0) \boldsymbol{\gamma}_{i}}{\left[\boldsymbol{\gamma}_{i}^{T} \mathbf{R}_{\dot{y} \dot{y}}(0) \boldsymbol{\gamma}_{i}\right]^{2}}
$$

Com algumas manipulações algébricas, a Eq. (7-6) se transforma no problema de autovalor

$$
\mathbf{R}_{y y}(0) \boldsymbol{\gamma}_{i}=\lambda_{i} \mathbf{R}_{\dot{y} \dot{y}}(0) \boldsymbol{\gamma}_{i}, \quad i=1, \ldots, n_{o} .
$$

Supondo que $\mathbf{R}_{y y}(0)$ e $\mathbf{R}_{\dot{y}}(0)$ sejam matrizes simétricas e positiva-definida, o problema acima gera $n_{o}$ diferentes autovalores e autovetores. Para calcular as frequências naturais e modos do sistema, é preciso que os problemas de autovalor definidos pelas equações (2-37) e (7-7) sejam relacionados. Para isso, utiliza-se novamente a suposição de estacionalidade dos sinais e a respectiva propriedade para as funções de correlação, definida pela Eq. (5-14).

Substituindo a matriz $\mathbf{R}_{\dot{y} \dot{y}}(0)$ por $-\mathbf{R}_{\ddot{y} y}(0)$, a Eq. (7-7) se transforma em

$$
\mathbf{R}_{y y}(0) \boldsymbol{\gamma}_{i}=-\lambda_{i} \mathbf{R}_{\ddot{y} y}(0) \boldsymbol{\gamma}_{i}, \quad i=1, \ldots, n_{o} .
$$

Utilizando o modelo de um sistema MDoF conservativo definido pela Eq. (2-35), onde o vetor do deslocamento real $\mathbf{q}(t)$ é substituído pelo observado $\mathbf{y}(t)$, e supondo que a matriz de massa $\mathcal{M}$ seja inversível, pode-se expressar a aceleração do sistema como sendo

$$
\ddot{\mathbf{y}}(t)=-\mathcal{M}^{-1} \mathcal{K} \mathbf{y}(t)+\mathcal{M}^{-1} \mathbf{u}(t)
$$

Aplicando a expressão acima na definição de $\mathbf{R}_{\ddot{y} y}(0)$, a equação (7-8) se 
transforma em

$$
\begin{aligned}
\mathbf{R}_{y y}(0) \boldsymbol{\gamma}_{i} & =-\lambda_{i} \mathbf{R}_{\ddot{y y y}}(0) \boldsymbol{\gamma}_{i} \\
& =-\lambda_{i} \mathbf{E}\left[\ddot{\mathbf{y}}(t) \mathbf{y}^{T}(t)\right] \boldsymbol{\gamma}_{i} \\
& =-\lambda_{i} \mathbf{E}\left[\left\{-\mathcal{M}^{-1} \mathcal{K} \mathbf{y}(t)+\mathcal{M}^{-1} \mathbf{u}(t)\right\} \mathbf{y}^{T}(t)\right] \boldsymbol{\gamma}_{i} \\
& =\lambda_{i} \mathcal{M}^{-1} \mathcal{K} \mathbf{E}\left[\mathbf{y}(t) \mathbf{y}^{T}(t)\right] \boldsymbol{\gamma}_{i}-\lambda_{i} \mathcal{M}^{-1} \mathbf{E}\left[\mathbf{u}(t) \mathbf{y}^{T}(t)\right] \boldsymbol{\gamma}_{i} \cdot(7-10)
\end{aligned}
$$

Como o vetor de força é composto por ruídos brancos, ele não é correlacionado com $\mathbf{y}(t)$ em um mesmo instante de tempo. O termo $\mathbf{E}\left[\mathbf{u}(t) \mathbf{y}^{T}(t)\right]$ se torna então zero, simplificando a Eq. (7-10) para

$$
\mathbf{R}_{y y}(0) \boldsymbol{\gamma}_{i}=\lambda_{i} \mathcal{M}^{-1} \mathcal{K} \mathbf{R}_{y y}(0) \boldsymbol{\gamma}_{i}, \quad i=1, \ldots, n_{o} .
$$

Ao comparar a Eq. (7-11) com a Eq. (2-37), percebe-se que os problemas de autovalor são semelhantes. Os autovalores e autovetores destes dois problemas são então relacionados pela equação

$$
\left\{\begin{array}{l}
\omega_{i}=\frac{1}{\sqrt{\lambda_{i}}} \\
\boldsymbol{\psi}_{i}=\mathbf{R}_{y y}(0) \boldsymbol{\gamma}_{i}
\end{array} \quad, \quad i=1, \ldots, n_{o} .\right.
$$

A relação acima mostra como a frequência natural e o modo do sistema podem ser estimados a partir do método de SOD. Ao ser comparado com os outros métodos de identificação, o SOD é mais simples de ser implementado. Basta que as correlações $\mathbf{R}_{y y}(0)$ e $\mathbf{R}_{\dot{y} \dot{y}}(0)$ sejam calculadas, para que o problema de autovalor da Eq. (7-7) seja construído e resolvido, possibilitando assim a identificação dos parâmetros modais pela relação da Eq. (7-12).

No entanto, duas grandes desvantagens do SOD podem ser observadas pela Eq. (7-7). A primeira consiste no fato de que ambas as correlações serem calculadas com $\tau=0$ (sem defasagem entre os sinais). Como visto no capítulo 5, as correlação para este valor de $\tau$ são as mais afetadas pelos ruídos. Conclui-se então que o método SOD tem mais sensibilidade a ruído que os demais. Além disso, a segunda desvantagem consiste no fato de que apenas $n_{o}$ autovalores e autovetores podem ser calculados. Assim, a quantidade de parâmetros modais que se pode identificar está atrelado ao número de sensores utilizados. Essa segunda desvantagem é superada ao realizar a identificação do sistema repetidamente com as respostas filtradas por diferentes bandas de frequência, garantindo assim que existam menos modos no sinal que o número de respostas $n_{o}$ em todas as etapas de identificação.

Esse processo pode ser realizado utilizando um filtro passa banda que desliza pelo espectro. O movimento é feito de forma discreta com um incremento de frequência $\Delta f$, como mostrado pela Figura 7.1. É preciso que a 


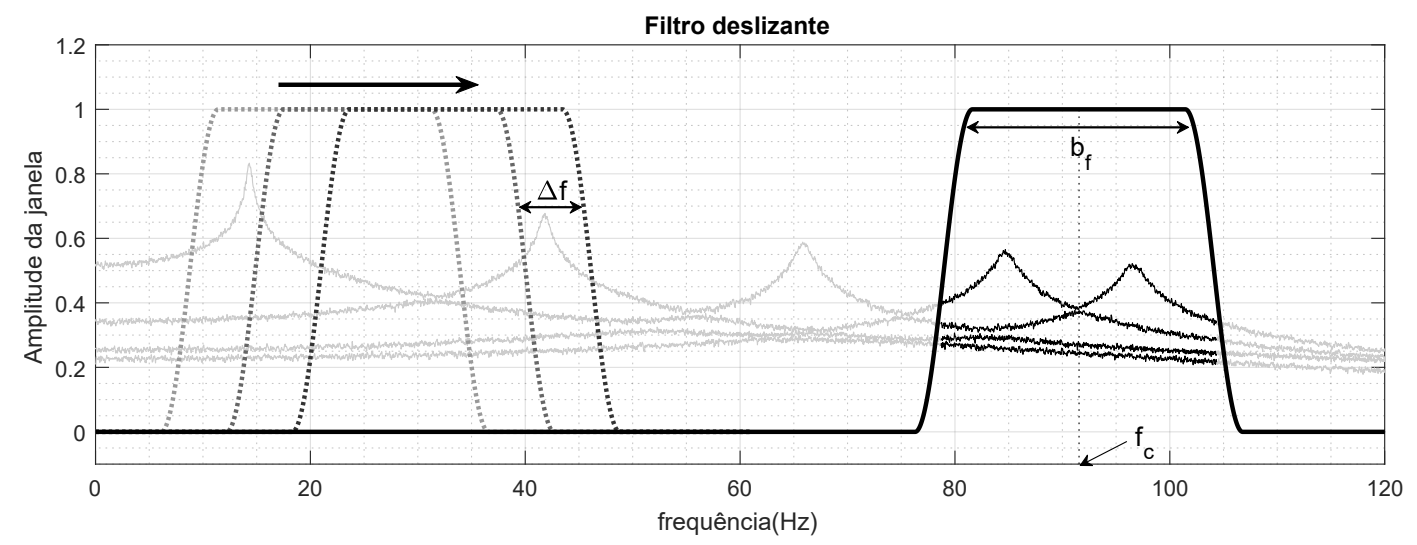

Figura 7.1: Ilustração do filtro passa banda que desliza sobre o espectro durante a identificação por SOD

espessura da banda $b_{f}$ também seja definida de forma que dentro dela nunca haja mais modos do que o número de respostas observadas (garantindo assim que todos os modos sejam identificados). A identificação é então realizada para cada uma das posições do filtro. Um digrama de estabilização pode ser construído marcando as frequências naturais estimadas em função da posição do filtro. Diferentemente dos diagramas anteriores, a quantidade de frequências naturais estimadas se mantém a mesma. O parâmetros que se altera, neste caso, é a posição do filtro, que é definida pela frequência central da banda. Um exemplo da implementação desse filtro e do diagrama de estabilização é mostrado a seguir no Exemplo 7.1.

\section{Exemplo 7.1}

Para ilustrar o processo de identificação apresentado acima, um exemplo numérico de uma viga engastada é utilizado. A geometria da estrutura e as respectivas propriedades do material são apresentadas na Figura 7.2. Uma força aleatória concentrada na ponta da viga foi escolhida para excitar o sistema. O sinal desta força consiste em um ruído branco com média zero, desvio padrão de $100 N$ e com distribuição Gaussiana.

Para simular a resposta desse sistema, uma discretização utilizando o método de elementos finitos (FEM) foi realizada. A malha criada contém 10 elementos de Euller-Bernoulli igualmente espaçados como mostrado na Figura 7.2. Em cada um dos elementos apenas dois nós foram utilizados. Como o método SOD foi desenvolvido para sistemas conservativos, nenhum fator de dissipação foi introduzido no modelo. Para se aproximar de um caso experimental, onde apenas 5 acelerômetros seriam utilizados, somente as respostas verticais dos nós 2,4,6,8 e 10 foram armazenados para a identificação. As respectivas respostas foram simuladas com uma frequência de amostragem igual a $5 \mathrm{kHz}$. 


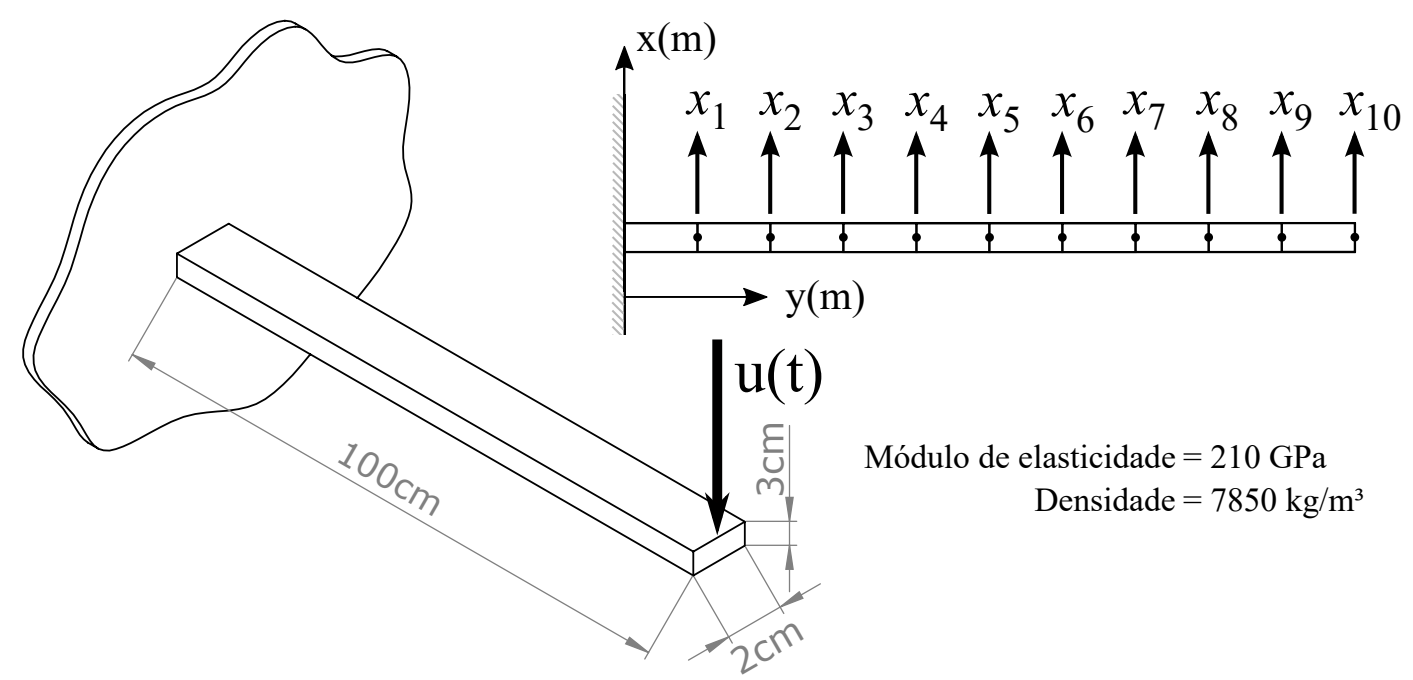

Figura 7.2: Geometria e propriedades do modelo numérico de uma viga engatada utilizada para ilustrar a identificação por SOD

O filtro passa banda que desliza pelo espectro foi projetado com a largura da banda $b_{f}$ igual a $500 \mathrm{~Hz}$. O incremento de frequência $\Delta f$ escolhido para mover o filtro tem valor igual a $2 \mathrm{~Hz}$. A posição inicial e final do filtro foram definidas pela frequência central da banda, com valores iguais a $-250 \mathrm{~Hz}$ e $1800 \mathrm{~Hz}$.

O diagrama de estabilização resultante para este exemplo é mostrado na Figura 7.3. Para cada posição do filtro, foi aplicado o método SOD que identificou cinco frequência naturais e modos (mesma quantidade de "sensores"na estrutura). Cada uma das frequências naturais estimadas foi marcada no diagrama em função da frequência central do filtro na posição em que se encontrava e foi classificada como estável ou instável. Uma frequência natural estável é aquela que possui um valor igual a de uma das frequência naturais estimadas pelo filtro na posição anterior. Como mostrado na Figura 6.14, linhas estáveis são construídas próximas as frequências naturais do sistema (linhas pontilhadas vermelhas). Isso ocorre porque, independente do valor da frequência central, se um modo do sistema estiver dentro da banda do filtro ele deve ser identificada.

Ao selecionar as frequências naturais estáveis mais próximas da linha pontilhada (que representa a frequência central do filtro) como resultados finais para os parâmetros do sistema, a Tabela 7.1 com os respectivos valores pode ser construída. Nesta mesma tabela também é mostrado os valores das frequências naturais calculadas analiticamente, pelo método dos elemento finitos e pelo método SOD sem o filtro deslizante. Nota-se que o modelo de elementos finitos resultou em frequências naturais próximas as analíticas, validando assim a discretização utilizada nas simulações. Percebe-se também que, além 


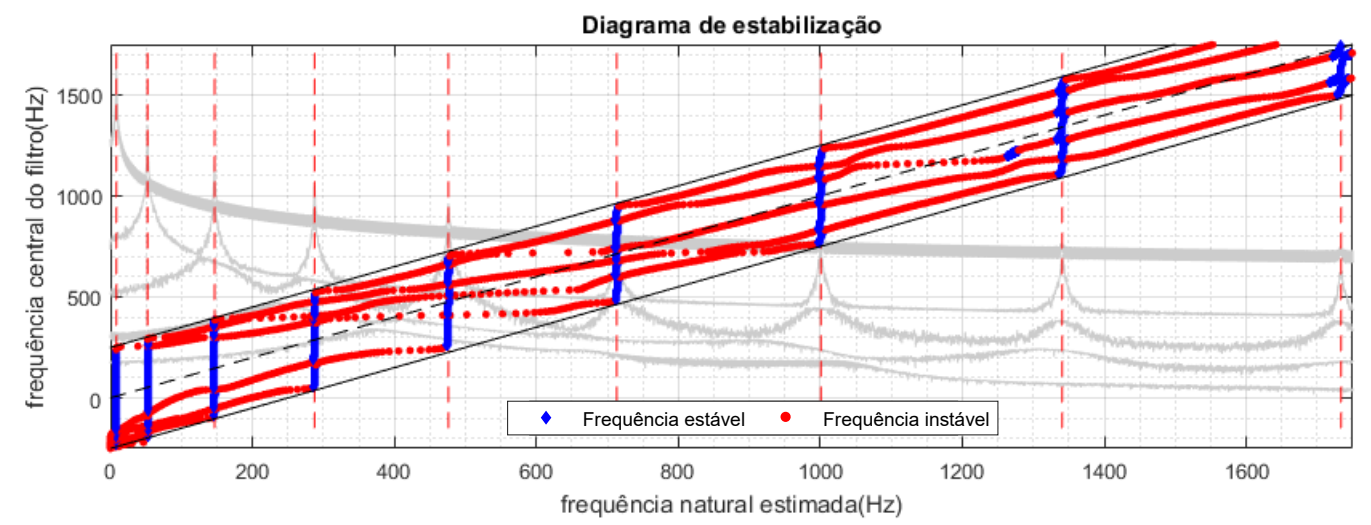

Figura 7.3: Diagrama de estabilidade obtida utilizando o filtro passa banda e o método de identificação SOD

de melhorar a identificação, a utilização do filtro passa banda permitiu a determinação de todas as frequências naturais, e não só as cinco primeiras. Os respectivo modos identificados pelo método SOD com o filtro deslizante são mostrados na Figura 7.4. Percebe-se que todos os oito modos foram perfeitamente identificados. (Fim do exemplo)

Tabela 7.1: Frequências naturais da viga identificadas pelo método SOD

\begin{tabular}{c|cccc}
\hline$\omega_{\mathbf{n}}$ & Analítica & FEM & SOD & $\begin{array}{c}\text { SOD } \\
\text { com filtro }\end{array}$ \\
\hline $1^{\mathrm{o}}$ & 8.355 & 8.355 & 8.475 & 8.342 \\
$2^{\mathrm{o}}$ & 52.361 & 52.363 & 54.780 & 52.335 \\
$3^{\mathrm{o}}$ & 146.613 & 146.650 & 154.475 & 146.556 \\
$4^{\mathrm{o}}$ & 287.299 & 287.575 & 307.973 & 287.540 \\
$5^{\mathrm{o}}$ & 474.931 & 476.127 & 500.543 & 476.537 \\
$6^{\mathrm{o}}$ & 709.462 & 713.292 & - & 713.283 \\
$7^{\mathrm{o}}$ & 990.902 & 1000.783 & - & 1000.290 \\
$8^{\mathrm{o}}$ & 1319.248 & 1340.774 & - & 1340.731 \\
\hline
\end{tabular}

\section{2}

\section{State-Variable Modal Decomposition}

Assim como o Smooth Orthogonal Decomposition, o método StateVariable Modal Decomposition (SVMD) consiste em uma extensão da Decomposição Ortogonal Própria. Ele foi desenvolvido para permitir que, além das frequências naturais e modos, o amortecimento da estrutura também fosse identificado. Desenvolvido por Feeny e Farooq [66], o SVMD é semelhante ao SOD só que com algumas modificações para ajustar o método ao modelo de espaço de estado.

Como proposto em [66], o SVMD permite apenas a identificação de sistemas oscilando livremente e por isso não pode ser aplicado em OMA. 


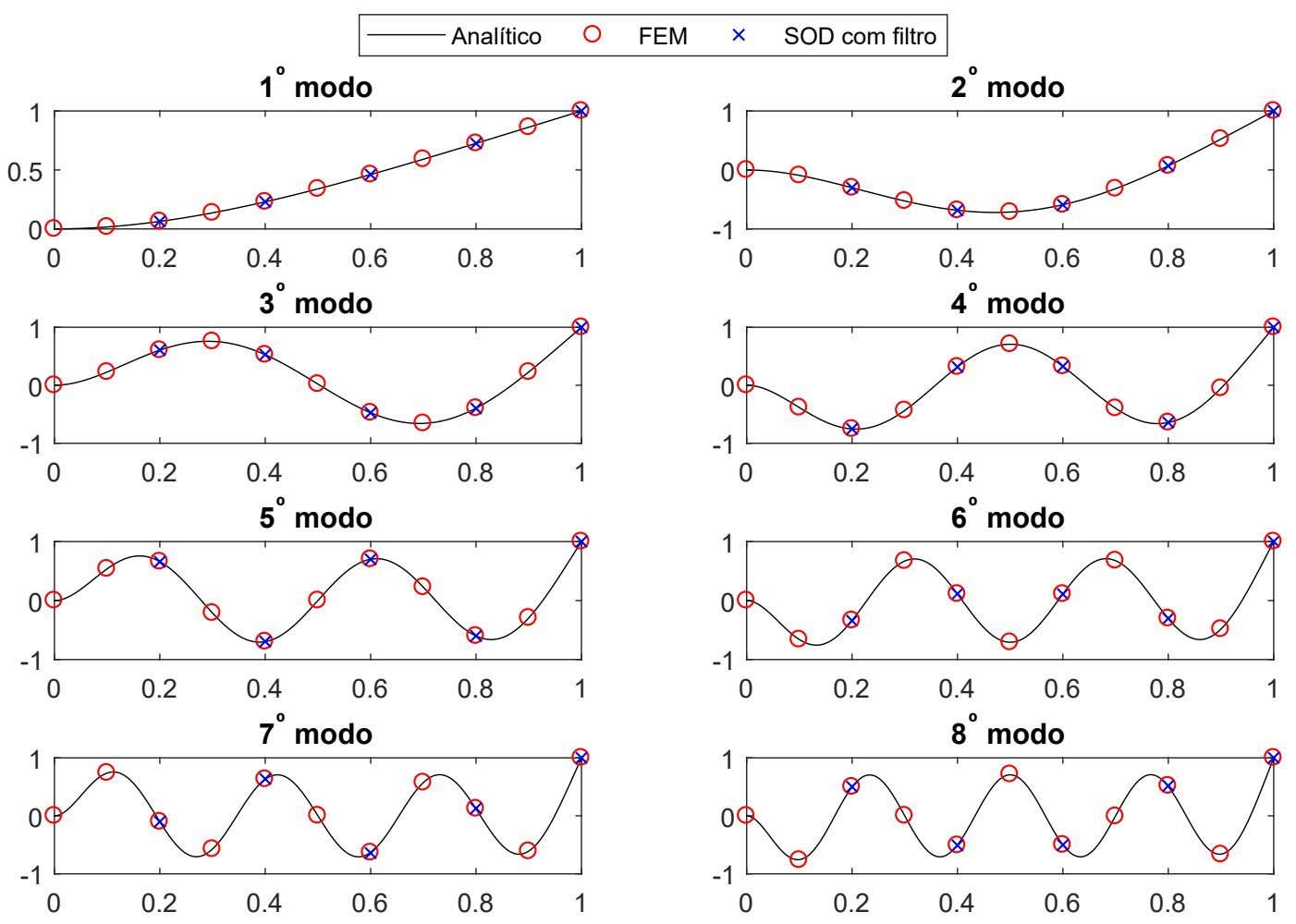

Figura 7.4: Formato do modos de vibração identificados pelo método SOD

Um nova implementação deste método foi proposto por Wagner et al. [67] para resolver essa limitação, permitindo que a identificação também seja feita para sistemas sob forçamento aleatório. Além disso, essa nova implementação permite que as incertezas dos parâmetros modais também sejam quantificadas.

O problema de autovalor que será utilizado no SVMD é semelhante ao do SOD definido pela Eq. (6-50), no entanto utilizando correlações dos estados do sistema. O resultado é a equação

$$
\mathbf{R}_{\dot{x} x}\left(\tau_{k}\right) \boldsymbol{\gamma}_{i}=\alpha_{i} \mathbf{R}_{x x}\left(\tau_{k}\right) \boldsymbol{\gamma}_{i}, \quad \tau_{k}>0, \quad i=1, \ldots, 2 n_{o},
$$

onde $\mathbf{x}=\left[\mathbf{y}^{T}(t) \dot{\mathbf{y}}^{T}(t)\right]^{T}$. A primeira diferença entre essa equação e a Eq. (7-7) consiste no fato da matriz de correlação $\mathbf{R}_{\dot{x} x}\left(\tau_{k}\right)$ só utilizar a derivada em um dos seus sinais. Isso ocorre porque o modelo de espaço de estado tem a ordem reduzida quando comparada à equações da dinâmica do sistema (utilizada no SOD). A segunda observação consiste no fato das matrizes de correlação não serem mais calculadas para $\tau_{k}=0$, e sim para qualquer valor positivo. Isso permite que os parâmetros modais sejam identificados diversas vezes, uma para cada valor de $\tau_{k}$. Assim, um histograma dos resultados podem ser construídos e as incertezas quantificadas. Além disso, pelo fato das correlações não serem mais calculadas para $\tau_{k}=0$, a sensibilidade do método em relação aos ruídos é melhorada.

Para mostrar como o problema de autovalor proposto pela Eq. (7-13) 
pode ser usado para identificar os parâmetros modais, uma relação com o problema de autovalor da Eq. (2-80) precisa ser estabelecida. Explicitando o termo da derivada do estado na Eq. (2-77), obtêm-se como resultado a equação

$$
\dot{\mathbf{x}}(t)=-\mathbf{A}^{-1} \mathbf{B} \mathbf{x}(t)+\mathbf{A}^{-1} \mathbf{f}(t) .
$$

Utilizando essa expressão e a definição das matrizes de correlação, o problema de autovalor da Eq. (7-13) pode ser reescrito na forma

$$
\begin{aligned}
\mathbf{R}_{\dot{x} x}\left(\tau_{k}\right) \boldsymbol{\gamma}_{i} & =\alpha_{i} \mathbf{R}_{x x}\left(\tau_{k}\right) \boldsymbol{\gamma}_{i} \\
\mathbf{E}\left[\mathbf{x}\left(t+\tau_{k}\right) \mathbf{x}^{T}(t)\right] \boldsymbol{\gamma}_{i} & = \\
\mathbf{E}\left[\left\{-\mathbf{A}^{-1} \mathbf{B} \mathbf{x}\left(t+\tau_{k}\right)+\mathbf{A}^{-1} \mathbf{f}\left(t+\tau_{k}\right)\right\} \mathbf{x}^{T}(t)\right] \boldsymbol{\gamma}_{i} & = \\
-\mathbf{A}^{-1} \mathbf{B} \mathbf{R}_{x x}\left(\tau_{k}\right) \boldsymbol{\gamma}_{i} & =
\end{aligned}
$$

Na última linha da Eq. (7-15) utilizou-se a hipótese do sistema ser causal, o que significa que os futuros forçamentos não são correlacionados com o presente estado (deslocamento e velocidade). Por isso o termo $\mathbf{E}\left[\mathbf{f}\left(t+\tau_{k}\right) \mathbf{x}^{T}(t)\right]$ é zero para $\tau_{k}>0$. Ao comparar a Eq. (7-15) com a Eq. (2-80), uma relação entre os autovalores e autovetores pode ser estabelecida na forma

$$
\left\{\begin{array}{l}
\lambda_{i}=\alpha_{i} \\
\boldsymbol{\phi}_{i}=\mathbf{R}_{x x}\left(\tau_{k}\right) \boldsymbol{\gamma}_{i}
\end{array}, \quad i=1, \ldots, 2 n_{o} .\right.
$$

Para que os parâmetros modais sejam identificados, basta então que o problema de autovalor da Eq. (7-13) seja resolvido para qualquer valor positivo de $\tau_{k}$ e os polos e $\phi_{i}$ estabelecidos pela relação da Eq. (7-17). O valor dos parâmetros modais são obtido então pelas equações

$$
\omega_{n i}=\sqrt{\lambda_{i} \lambda_{i}^{*}}, \quad \zeta_{i}=-\frac{\Re\left(\lambda_{i}\right)}{\omega_{n i}}, \quad \boldsymbol{\psi}_{i}=\left[\begin{array}{ll}
\mathbf{I} & 0
\end{array}\right] \boldsymbol{\phi}_{i}, \quad i=1, \ldots, 2 n_{o}
$$

Embora esse método identifique $2 n_{o}$ parâmetros modais, apenas $n_{o}$ valores são distintos já que o problema de autovalor da Eq. (7-13) resulta em autovalores e autovetores complexos conjugados. Teoricamente, os parâmetros modais poderiam ser identificados diversas vezes para qualquer valor positivo de $\tau_{k}$. No entanto, como as funções de correlação têm sua magnitude decaindo exponencialmente, existe um valor limite para $\tau_{k}$ antes que a identificação comece a ser prejudicada por ruídos (quando as correlações se aproximam de zero). Isso será melhor discutido no exemplo a seguir.

Para quantificar as incertezas dos parâmetros, basta que histogramas com resultados obtidos por todos os $\tau_{k}$ sejam construídos. 


\section{Exemplo 7.2}

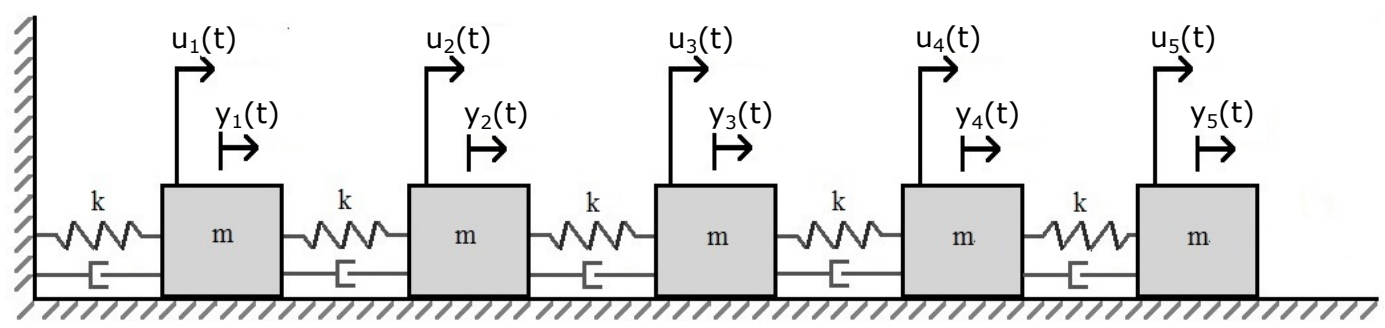

Figura 7.5: Sistema discreto de cinco graus de liberdade utilizado para ilustrar a identificação por SVMD

Neste exemplo, utiliza-se o modelo discreto apresentado pela Figura 7.5, onde $m=0.1 \mathrm{~kg}$ e $k=1000 \mathrm{~N} / \mathrm{m}$. A dissipação do sistema foi definida pelo fator de amortecimento modal, cujos valores foram escolhidos como sendo $\zeta_{i}=1.5 \%$. Os forçamentos $u_{i}(t)$ foram definidos como ruídos brancos não-correlacionados de distribuição Gaussiana e com desvio padrão igual a 100N. Para simular as respostas do sistema, utilizou-se uma frequência de amostragem igual a $3 \mathrm{kHz}$. Um total de 409.600 amostras foram geradas, resultando em 136,5 segundos de simulação. Para cada uma das resposta, adicionou-se um ruído branco com desvio padrão igual a $10 \%$ do desvio padrão das respectivas respostas. Isso foi realizado para simular um possível ruído causado pelo sensor durante as medições.

Em seguida, após as derivações necessárias, os vetores $\mathbf{x}(k)$ e $\mathbf{\mathbf { x }}(k)$ foram montados. As matrizes $\mathbf{R}_{x x}\left(\tau_{k}\right)$ e $\mathbf{R}_{\dot{x} x}\left(\tau_{k}\right)$ foram então calculadas para valores de $\tau_{k}$ entre 1 e 4096. Uma das funções de correlação é mostrada pela Figura 7.6. Como previsto, percebe-se que essa função tem o formato de decaimento exponencial. Através desse gráfico, pode-se determinar a quantidade de $\tau_{k}$ que será utilizado no método. A escolha desse parâmetro deve ser feita escolhendo a maior quantidade possível de $\tau_{k}$ sem que a função de correlação já tenha se aproximado de zero. Para valore altos de $\tau_{k}$, a correlação têm valores próximos de zero e por isso qualquer ruído passa a influenciar bastante no seu resultado. Neste exemplo, escolheu-se identificar os parâmetros modais utilizando os primeiros 1500 valores de $\tau_{k}$. Assim, a identificação pode ser feita 1500 vezes utilizando apenas uma simulação. Como resultado, um histograma das frequências naturais e fatores de amortecimento podem ser construídos como mostrado na Figura 7.7. Além dos histogramas, um envelope dos modos também foi construído para ilustrar a incerteza desse parâmetro.

Percebe-se que, caso o valor médio do histograma seja escolhido como valor final dos parâmetros, uma boa identificação foi realizada. Nota-se que, a medida que a ordem do modos aumentam, maiores são os desvios padrões 


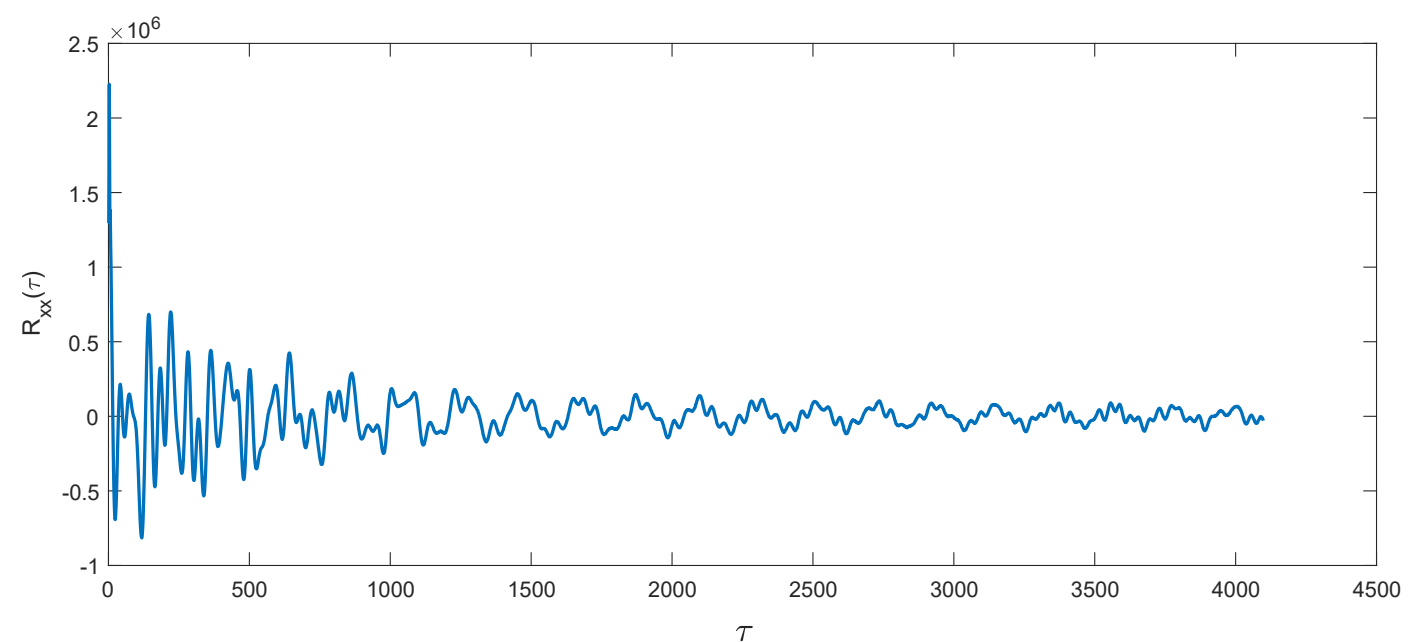

Figura 7.6: Exemplo de uma das funções de correlação obtidas na matriz $\mathbf{R}_{x x}\left(\tau_{k}\right)$

dos histogramas. Isso significa que mais incerto é o valor dos parâmetros identificados. (Fim do exemplo) 

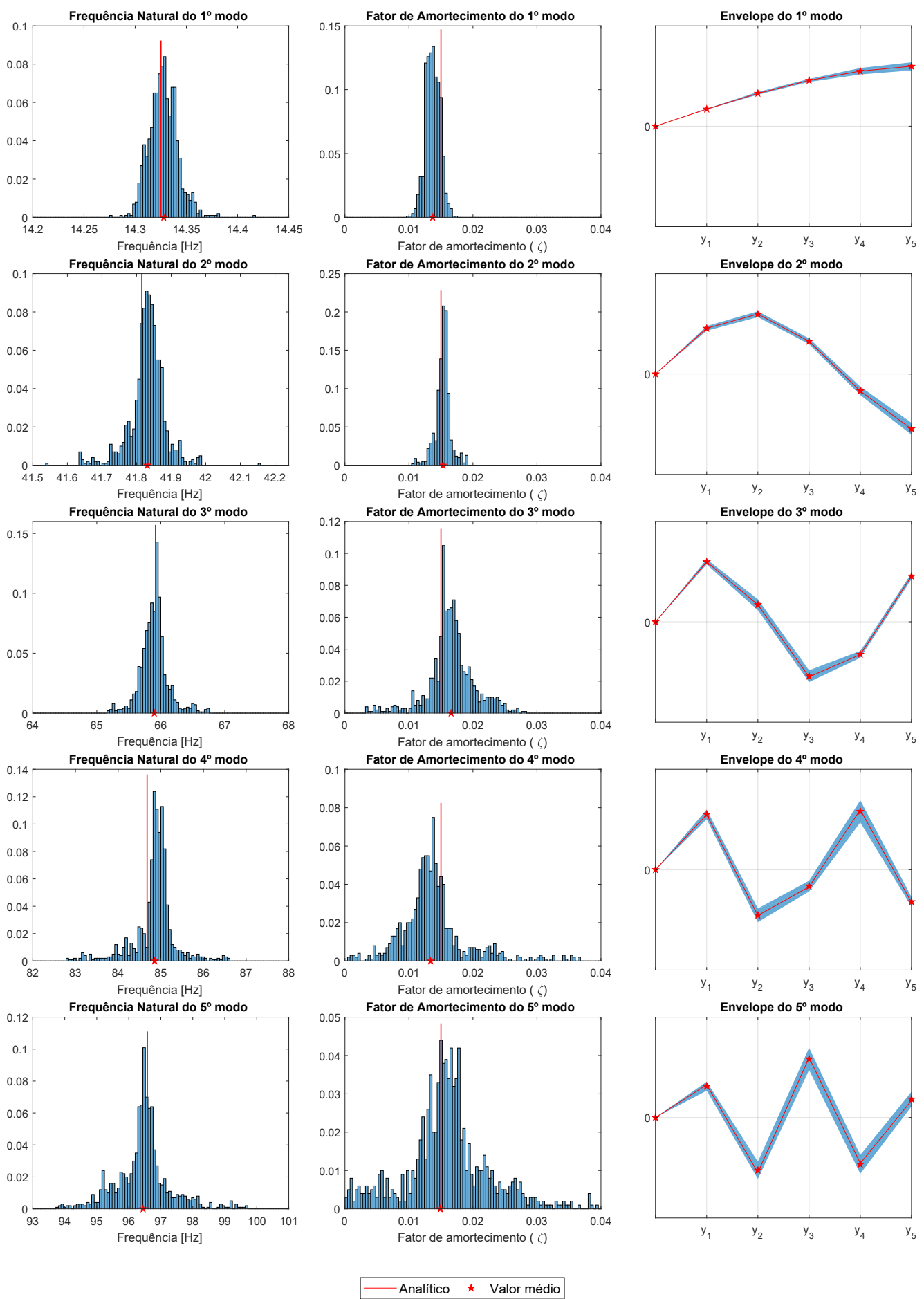

Figura 7.7: Histograma e envelope dos parâmetros modais identificados pelo método de SVMD 


\section{8 \\ Conclusões}

O principal objetivo desta dissertação foi definir o que é análise modal operacional (OMA) e como essa técnica pode ser aplicada experimentalmente. Neste documento, OMA foi definida como sendo o processo de identificação dos parâmetros modais de um sistema quando excitado pelo ambiente, onde somente as suas respostas são conhecidas. Para os forçamentos, supôs-se que eles eram aleatórios e que tinham energia distribuída por todo espectro analisado. Dessa forma, garante-se que todos os modos são excitados e consequentemente identificados.

Na primeira etapa desta dissertação, realizou-se um estudo sobre como sistemas lineares podem ser caracterizados em função dos seus parâmetros modais. No capítulo 2, mostrou-se que sinais de força podem ser processados e transformados em sinais de resposta. Isso pôde ser realizado através de duas funções: a de resposta ao impulso unitário (domínio do tempo) e a de resposta em frequência (domínio da frequência). Ambas funções são importantes porque podem ser escritas em função dos parâmetros modais do sistema.

Utilizando esses resultados, um estudo semelhante foi realizado no capítulo 3 para sistemas excitados por forças aleatórias. Quando modelado por processos estacionários, as estatísticas do forçamento puderam ser relacionadas com as estatísticas dos deslocamentos através dos parâmetros do sistema. Utilizando as hipóteses de forçamento por ruído branco ou de coordenadas modais não correlacionadas, mostrou-se que as funções de correlação e de densidade espectral de potência poderiam ser utilizadas como fonte de informação para os métodos identificação. Isso porque ambas as funções puderam ser decompostas em relação ao parâmetros modais. Como o foco desta dissertação foi o estudo dos métodos de identificação no domínio do tempo, as funções de correlação foram analisadas com mais detalhes. Ainda no capítulo 3 mostrou-se que essas funções podiam ser escritas como um somatório de oscilações cujas magnitudes decaiam exponencialmente.

Já no capítulo 4, utilizou-se modelagens discretas para representar um sistema. Ao adotar o modelo de espaço de estado discreto no tempo, as funções de correlação puderam ser escritas de forma semelhante às funções de resposta ao impulso (através de parâmetros de Markov). O modelo ARMA também foi 
apresentado representar um sistema. Para ambos os casos, mostrou-se como os parâmetros modais poderiam ser extraídos desses modelos.

A primeira importante contribuição desta dissertação foi a realização de um estudo sobre a deformação causada pelos ruídos de medição nas funções de correlação. No capítulo 5, analisou-se essa deformação em três diferentes casos: quando a correlação foi estimada diretamente do sinal ruidoso, quando a correlação foi estimada após uma derivação e quando a correlação foi estimada após uma integração. Para o segundo e terceiro caso, utilizou-se deferentes filtros para processar os sinais. Em todos os casos constatou-se que a deformação causada pelo ruído era igual a sua própria função de correlação. Além disso, concluiu-se que quando $\tau_{k}=0$, as funções de correlação são mais afetada pelo ruído. Esse resultado foi utilizado para melhor entender porque os métodos SOD e SVMD eram mais sensíveis ao ruído.

Utilizando os resultados dos capítulos 2,3 e 4, diferentes métodos de identificação puderam ser apresentados. No capítulo 6 discutiu-se três dos métodos de identificação mais populares no domínio do tempo. Em todos eles, matrizes de Hankel precisaram ser definidas e utilizadas. Diferentes estruturas foram identificadas para validar a implementação dos algorítimos em Matlab. Além disso, os exemplos serviram para ilustrar os procedimentos experimentais necessários para uma correta identificação. Diagramas de estabilização também foram apresentados e utilizados no procedimento de análise dos resultados. Para o método AR/PR, identificou-se o modelo de um prédio de dois andares. Neste método, mostrou-se que a ordem do sistema identificado é dependente do número de matrizes utilizadas no modelo ARMA. Já para o método ERA, a bancada de uma ponte suspensa foi utilizada para ilustrar a identificação. Nesta bancada um total de 11 modos foram identificados. Por conta de dois modos pouco espaçados, pode-se mostrar como a identificação nesses casos pode se torna difícil. Por último, mostrou-se a identificação de uma pá eólica utilizando o método SSI. Dois algorítimos de foram mostrados, mas apenas o covariancedriven foi utilizado no exemplo. Com ele um total de 6 modos puderam ser bem identificados. Esse exemplo permitiu ilustrar como a identificação com múltiplos testes pode ser feita. Além disso, pode-se mostrar como a escolha dos sensores pode afetar os resultados, já que o primeiro modo não foi bem identificado por estar fora da banda útil de frequência dos acelerômetros selecionados.

A segunda importante contribuição desta dissertação inclui a apresentação de duas novas implementações de métodos de decomposição ortogonais no capítulo 7. Essas implementações são inéditas na literatura e permitem solucionar alguns dos problemas desses métodos. Em relação ao problema da 
quantidade de modos identificados, um filtro passa banda que desliza sobre o espectro dos sinais das respostas foi proposto no método SOD. Com ele, a quantidade de modos identificados não fica mais atrelada ao número de sensores utilizados. Além disso, um novo tipo de diagrama de estabilização pôde ser construído e apresentado. Para solucionar o problema da sensibilidade em relação aos ruídos, generalizou-se as matrizes de correlação no método SVMD com $\tau=0$ para $\tau$ com qualquer valor positivo. Dessa forma, correlações menos afetadas pelo ruído puderam ser utilizadas. Além disso a identificação dos parâmetros pôde ser feita diversas vezes utilizando apenas um único teste. Assim, um histograma dos parâmetros modais identificados também pode ser construído e as respectivas incertezas quantificadas.

Como contribuição ao laboratório de vibrações, uma interface gráfica também foi construída para realizar testes de análise modal operacional. Programado em Matlab, essa interface possibilitou o controle de placas de aquisições comerciais e permite a visualização os resultados em tempo real. No anexo A encontra-se o manual completo desta interface.

Para futuros trabalhos, outros métodos existentes na literatura podem ser incorporados a este estudo. Exemplos são os métodos ARMA [14], Ibrahim Time Domain [68], Decomposição no domínio da frequência [69] e o LSCF [20]. Além disso, um importante aspecto em identificação de sistemas que não foi tratado nesta dissertação é a incerteza nos valores dos parâmetros. Ao repetir um experimento em uma estrutura, os valores dos parâmetros identificados dificilmente se repete. Os principais motivos para essa variação são: quantidade finita de dados, ruídos nos sinais e erros nos modelos do sistema e do forçamento. Uma boa forma de abordar a incerteza dos parâmetros é interpreta-los como variáveis aleatórias, onde a incerteza é quantificada através de suas distribuições de probabilidade. Isso pode ser realizado utilizando a inferência Bayesiana [21]. 


\section{Referências bibliográficas}

[1] HAYLEN, W.; LAMMENS, S.; SASS, P. Modal analysis theory and testing. Department of Mechanical Engineering, Kathoelieke Universiteir Leuven, 1997.

[2] HE, J.; FU, Z.F. Modal Analysis. Butterworth-Heinemann, Jordan Hill, Oxford, 2001.

[3] DALLARD, P.; FITZPATRICK, A. J.; FLINT, A.; BOURVA, S.; SMITH, R. M. R.; WILLFORD, M. The london millennium footbridge. Shock and Vibration, 20:493-502, 2013.

[4] MAIA, N. M. M.; SILVA, J. M. M. Theoretical and Experimental Modal Analysis. Research Studies Press LTD., Somerset,England, 1997.

[5] EWINS, D. J. Modal Testing: Theory and Practice. Wiley, 1984.

[6] FRISWELL, M.; MOTTERSHEAD, J.E. Finite Element Model Updating in Structural Dynamics. Springer Netherlands, 2010.

[7] MARWALA, T. Finite Element Model Updating Using Computational Intelligence Techniques: Applications to Structural Dynamics. Springer Science \& Business Media, 2010.

[8] PEeters, B.; CliMENT, H.; DIEGO, R.; ALBA, J.; AHLQUIST, J. R.; CARREÑO, J. M.; HENDRICX, W.; REGA, A.; GARCÍA, G.; DEWEER, J.; DEBILLE, J. Modern solutions for ground vibration testing of large aircraft. In: PROCEEDINGS OF THE XXVI IMAC, p. 4-7, 2008.

[9] WEnZEL, H.; PICHLER, D.. Ambient Vibration Monitoring. John Wiley \& Sons, West Sussex, UK, 2005.

[10] FERRAR, C. R.; WORDEN, K. Structural Health Monitoring. John Wiley \& Sons, West Sussex, UK, 2013.

[11] REYNDERS, E.; WURSTEN, G.; DE ROECK, G. Output-only structural health monitoring in changing environmental conditions by means of nonlinear system identification. Structural Health Monitoring, 13:82-93, 2014. 
[12] MAGAlhÃES, F. M. R. L. Operational Modal Analysis for testing and monitoring of bridges and special structures. $\mathrm{PhD}$ thesis, Universidade do Porto, Porto, 2010.

[13] RAINIERI, C.; FABBROCINO, G. Operational Modal Analysis of Civil Engineering Structures. Springer Science and Business Media, New York, 2014.

[14] ANDERSEN, P.; BRINCKER, R.; KIRKEGAARD, P. H. Identification of Civil Engineering Structures using Vector ARMA Models. Aalborg: Dept. of Building Technology and Structural Engineering, Aalborg University, 1998.

[15] RODRIGUES, J. Identificação Modal Estocástica: Métodos de análise e aplicações em estruturas de engenharia civil. Tese de doutorado, Universidade do Porto, Porto, 2004.

[16] CABBOI, A. Automatic operational modal analysis: Challenges and applications to historic structures and infrastructures. $\mathrm{PhD}$ thesis, Università degli Studi di Cagliari, Cagliari, 2013.

[17] CHATZI, E. N.; PAPADIMITRIOU, C. Identification Methods for Structural Health Monitoring. Springer, 2016.

[18] YAUN, F.G. Structural Health Monitoring (SHM) in Aerospace Structures. Elsevier, Cambridge, USA, 2016.

[19] CURSI, E. S.; SAMPAIO, R. Uncertainty Quantification and Stochastic Modeling with Matlab. ISTE Press, 2015.

[20] CAUBERGHE, B. Applied Frequency-Domain System Identification in the Field of Experimental and Operational Modal Analysis. PhD thesis, Vrije Universiteit, Brussel, 2004.

[21] AU, S. K.. Operational Modal Analysis: Modeling, Bayesian Inference, Uncertainty Laws. Springer, 2017.

[22] ADHIKARI, S.. Structural Dynamic Analysis with Generalized Damping Models. ISTE Ltd and John Wiley \& Sons, Uk and USA, 2014.

[23] INMAN, D.J. Engineering vibration. Englewood Cliffs, NJ, Prentice Hall, 2014.

[24] GENTA, G. Vibration Dynamics and Control. Springer Science and Business Media, New York, NY, USA, 2009. 
[25] GATTI, P. L.. Applied Structural and Mechanical Vibrations: Theory and Methods. CRC Press, 2014.

[26] BRANDT, A. Noise and Vibration Analysis: Signal Analysis and Experimental Procedures. John Wiley \& Sons, West Sussex, UK, 2011.

[27] BRINCKER, R.; VENTURA, C. Introduction to Operational Modal Analysis. John Wiley \& Sons, West Sussex, UK, 2015.

[28] LIMA, R.; SAMPAIO, R. Analysis of random signals: First part of a course on random vibration. In: COURSE PRESENTED IN THE XVII INTERNATIONAL SYMPOSIUM ON DYNAMIC PROBLEMS OF MECHANICS (DINAME), 2017.

[29] SAMPAIO, R; CATALDO, E; BRANDÃO, A. Análise e processamento de sinais. 22. SBMAC: Notas de aula em matemática aplicada, 2006.

[30] LIMA, R.; SAMPAIO, R. Processing of random signals: Second part of a course on random vibration. In: COURSE PRESENTED IN THE XVII INTERNATIONAL SYMPOSIUM ON DYNAMIC PROBLEMS OF MECHANICS (DINAME), 2017.

[31] SOIZE, C.. Fundamentals of Random Signal Analysis: Application to Modal Identification in Structural Dynamics. Manustrito não puplicado, 1997.

[32] OPPENheIM, A. V.; WILLSKY, A. S. Signals and Systems. Pearson Education Limited, Edinburgh Gate, Harlow, 1996.

[33] BRINCKER, R.; ZHANG, L.; ANDERSEN, P. Modal identification of output-only systems using frequency domain decomposition. Smart Materials and Structures, 10:441-445, 2001.

[34] BRINCKER, R.; ZHANG, L.; ANDERSEN, P. Modal identification from ambient responses using frequency domain decomposition. In: PROCEEDINGS OF THE INTERNATIONAL MODAL ANALYSIS CONFERENCE, p. 625-630, 2000.

[35] BRINCKER, R.; ZHANG, L.; ANDERSEN, P. Modal identification of output-only systems using frequency domain decomposition. Smart Materials and Structures, 10(3):441-445, 2000.

[36] BRINCKER, R.; ZHANG, L. Frequency domain decomposition revised. In: PROCEEDINGS OF THE INTERNATIONAL OPERATIONAL MODAL ANALYSIS CONFERENCE, p. 615-626, 2009. 
[37] JUANG, J.N. Applied System Identification. PTR Prentice-Hall, Englewood Cliffs, New Jersey, 1994.

[38] OVERSCHEE, P. V.; DE MOOR, B.. Subspace Identification for Linear System: Theory - Implementation - Applications. Kluwer Academic Publishers, Katholieke Universiteit Leuven, Belgium, 1996.

[39] REYNDERS, E.; DE ROECK, G. New Trends in Vibration Based Structural Health Monitoring, chapter 3. Subspace identification for operational modal analysis, p. 55-106. Springer-Verlag Wien, 2010.

[40] PINTELON, R.; SCHOUKENS, J. Real time integration and differentiation of analog-signals by means of digital filtering. IEEE Transactions on Instrumentation and Measurement, 39:923-927, 1990.

[41] PARKS, T. W., MCCLELLAN, J. Chebyshev approximation for nonrecursive digital filters with linear phase. IEEE Transactions on Circuit Theory, CT19:189-194, 1972.

[42] OPPENhEIM, A. V.; SCHAFER, R. W.; BUCK, J. R. Discrete-Time Signal Processing. Pearson Education Limited, Edinburgh Gate, Harlow, 1999.

[43] BEndAT, J. S.; PIERSOL, A. G. Random Data: Analysis and Measurement Procedures. John Wiley \& Sons, Hoboken, New Jersey, 2010.

[44] WIRSCHING, P. H.; PAEZ, T. L.; ORTIZ, K. Random Vibrations: Theory and Practice. Dover Publications, Mineola, New York, 2006.

[45] FONSECA, C.A.; WAGNER, G.; LIMA, R.; SAMPAIO, R. Robust identification and passive control of vibration of a test rig under uncertain conditions. Mecánica Computacional, XXXIII:1767-1781, 2014.

[46] JUANG, J.N.; PAPPA, R.S. An eigen system realization algorithm for modal parameter identification and modal reduction. Journal of Guidance, Control, and Dynamics, 8(5):620-627, 1985.

[47] PAPPA, R.S.; ELLIOTT, K.B.; SCHENK, A. Consistent-mode indicator for the eigensystem realization algorithm. Journal of Guidance, Control, and Dynamics, 16(5):852-858, 1993.

[48] PAPPA, R.S. Eigensystem realization algorithm, user's guide for vax/vms computers. NASA Technical Memorandum 109066, 1994. 
[49] REYNDERS, E.; WURSTEN, G.; DE ROECK, G. Uncertainty quantification in operational modal analysis with stochastic subspace identification. Mechanical Systems and Signal Processing, 66-67:13-30, 2016.

[50] MELLINGER, P.; DÖHLER, M.; MEVEL, L.. Variance estimation of modal parameters from output-only and input/output subspacebased system identification. Jornal of Sound and Vibration, 379:1-27, 2016.

[51] WAGNER, G.; FOINY, D.; LIMA, R.; SAMPAIO, R.. Operational modal analysis under wind load using stochastic subspace identification. In: PROCEEDINGS OF THE XVII INTERNATIONAL SYMPOSIUM ON DYNAMIC PROBLEMS OF MECHANICS (DINAME), 2017.

[52] FEENY, B.F.; KAPPAGANTU, R. On the physical interpretation of proper orthogonal modes in vibration. Journal of Sound and Vibration, 211(4):157-160, 1998.

[53] FEENY, B.F. On the proper orthogonal modes and normal modes of continuous vibration systems. Journal of Sound and Vibration, 124(1):157-160, 2002.

[54] BELLIZZI, S.; SAMPAIO, R. Poms analysis of randomly vibrating systems obtained from karhunen-loève expansion. Journal of Sound and Vibration, 297:774-793, 2006.

[55] BELLIZZI, S.; SAMPAIO, R. Karhunen-loève modes obtained from displacement and velocity fields: Assessments and comparisons. Mechanical Systems and Signal Processing, 23:1218-1222, 2009.

[56] BELLIZZI, S.; SAMPAIO, R. Smooth karhunen-loève decomposition to analyze randomly vibrating systems. Mechanical Systems and Signal Processing, 325:491-498, 2009.

[57] SAMPAIO, R.; BELLIZZI, S. Analysis of nonstationary random processes using smooth decomposition. Journal of Mechanics of Materials and Structures, 6:1137-1151, 2011.

[58] BELLIZZI, S.; SAMPAIO, R. Reduced models based on smooth decomposition for random mechanical systems. International Review of Mechanical Engineering, 6:74-84, 2012. 
[59] BELLIZZI, S.; SAMPAIO, R. Smooth decomposition of random fields. Journal of Sound and Vibration, 331:3509-3520, 2012.

[60] BELLIZZI, S.; SAMPAIO, R. Analysis of stationary random vibrating systems using smooth decomposition. Shock and Vibration, 20:493502, 2013

[61] FOINY, D.; WAGNER, G.; LIMA, R.; SAMPAIO, R.. Dynamical system identification with smooth decomposition. In: PROCEEDINGS OF THE XVII INTERNATIONAL SYMPOSIUM ON DYNAMIC PROBLEMS OF MECHANICS (DINAME), 2017.

[62] WAGNER, G.; FOINY, D.; LIMA, R.; SAMPAIO, R. The robust smooth orthogonal decomposition method for operational modal analysis. In: PROCEEDINGS OF THE 7TH IOMAC, 2017.

[63] CHELIDZE, D.; ZHOU, W. Smooth orthogonal decomposition-based vibration mode identification. Journal of Sound and Vibration, 292:461473, 2006

[64] FAROOQ, U.; FEENY, B.F.. Smooth orthogonal decomposition for modal analysis of randomly excited systems. Journal of Sound and Vibration, 316:137-146, 2008.

[65] BELLIZZI, S.; SAMPAIO, R. The smooth decomposition as a nonlinear modal analysis tool. Mechanical Systems and Signal Processing, 64-65:245-256, 2015.

[66] FEENY, B.F.; FAROOQ, U. A nonsymetric state-variable decomposition for modal analysis. Journal of Sound and Vibration, 310(4-5):792800,2008

[67] WAGNER, G.; FOINY, D.; LIMA, R.; SAMPAIO, R. A new robust implementation of SVMD for output-only modal analysis. In: PROCEEDINGS OF THE 3RD EMI-IC, 2017.

[68] RODRIGUES, J.; BRICKER, R.. Application of the random decrement technique in operational modal analysis. In: 1ST INTERNATIONAL OPERATIONAL MODAL ANALYSIS CONFERENCE, Copenhagen, Denmark, 2005.

[69] RODRIGUES, J.; BRICKER, R.; ANDERSEN, P. Improvent of frequency domain output-only modal identification from the application of 
the random decrement technique. In: IMAC-XXII: CONFERENCE \& EXPOSITION ON STRUCTURAL DYNAMICS, Dearborn, Michigan, 2004. 


\section{Sistema de aquisição de dados}

O objetivo deste apêndice é detalhar o sistema de aquisição de dados desenvolvido e utilizado no Laboratório de Vibrações para análise modal operacional. Será apresentado a seguir tanto os equipamentos utilizados quanto o software desenvolvido.

Em relação aos equipamentos, os sensores e os respectivos condicionadores de sinal podem variar dependendo da estrutura testada. Parâmetros como peso, rigidez e geometria influenciam bastante na seleção dos sensores. Além disso, a banda de frequência analisada também pode restringir a utilização de um determinado sensor. Por exemplo, acelerômetros piezoelétricos costumam não funcionar bem para baixas frequências e por isso acelerômetros piezo resistivos são mais indicados. Independente do sensor escolhido, a sequência em que os equipamentos são utilizados não se altera. A Figura A.1 ilustra um típico set utilizado em OMA. A tabela A.1 mostra os equipamentos utilizados em cada uma das estruturas testadas nessa dissertação. Para o teste no prédio e na ponte, o condicionamento do sinal é feito pelo sensor.

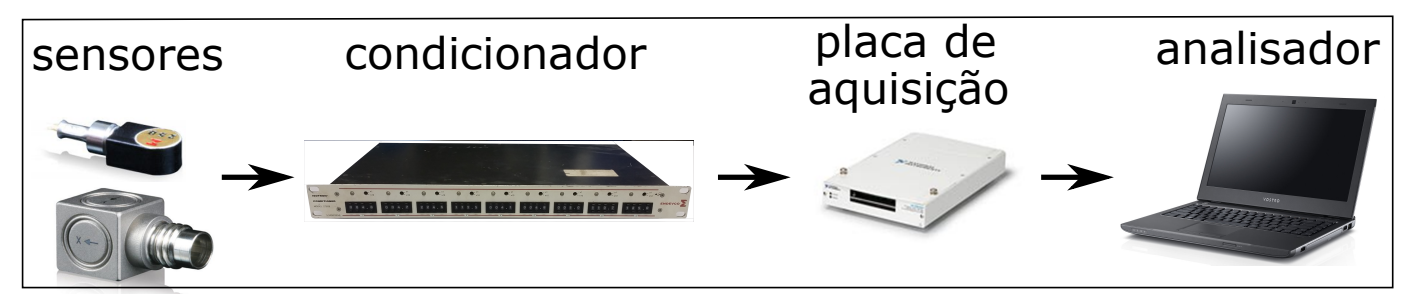

Figura A.1: Diagrama de estabilidade obtida utilizando o filtro passa banda e o método de identificação SOD

Tabela A.1: Equipamentos utilizados em cada um dos testes

\begin{tabular}{ccccc}
\hline Estrutura & Sensores & Condicionador & $\begin{array}{c}\text { Placa de } \\
\text { aquisição }\end{array}$ & Analizador \\
\hline Prédio & LD1607-100 & - & NI USB-6229 & PC/Matlab \\
Ponte & MMA7341LC & - & NI USB-6229 & PC/Matlab \\
Pá eólica & Micro 25B & Endevco 2792B & NI USB-6229 & PC/Matlab \\
\hline
\end{tabular}

Uma importante contribuição desta dissertação foi utilizar o Matlab para realizar a comunicação entre a placa de aquisição e o analisador (computador). 
Isso foi realizado através de uma interfase gráfica desenvolvida no Laboratório de Vibrações que permite um fácil e intuitivo processo de aquisição. As principais etapas são listadas a seguir.

1. Definir a frequência de aquisição, o número de amostras por teste e o número de teste.

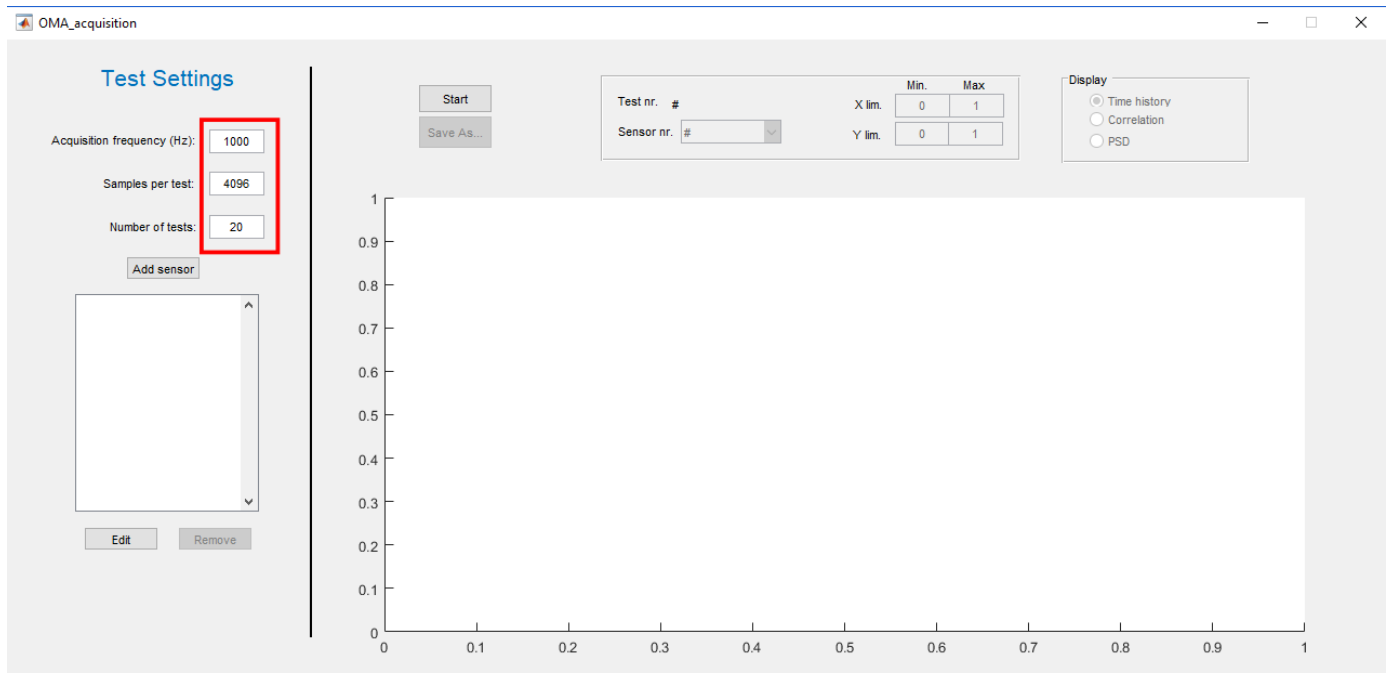

Figura A.2: Seleção dos parâmetros do teste no programa.

2. Definir os sensores que serão utilizados no teste. Para isso, é preciso definir o tipo de sinal que será amostrado (analógico, analógico com complementação de ponte ou digital), a placa de aquisição e a porta em que o sensor está conectado. Isso é realizado através da janela que se abre ao clicar no botão "Add sensor".

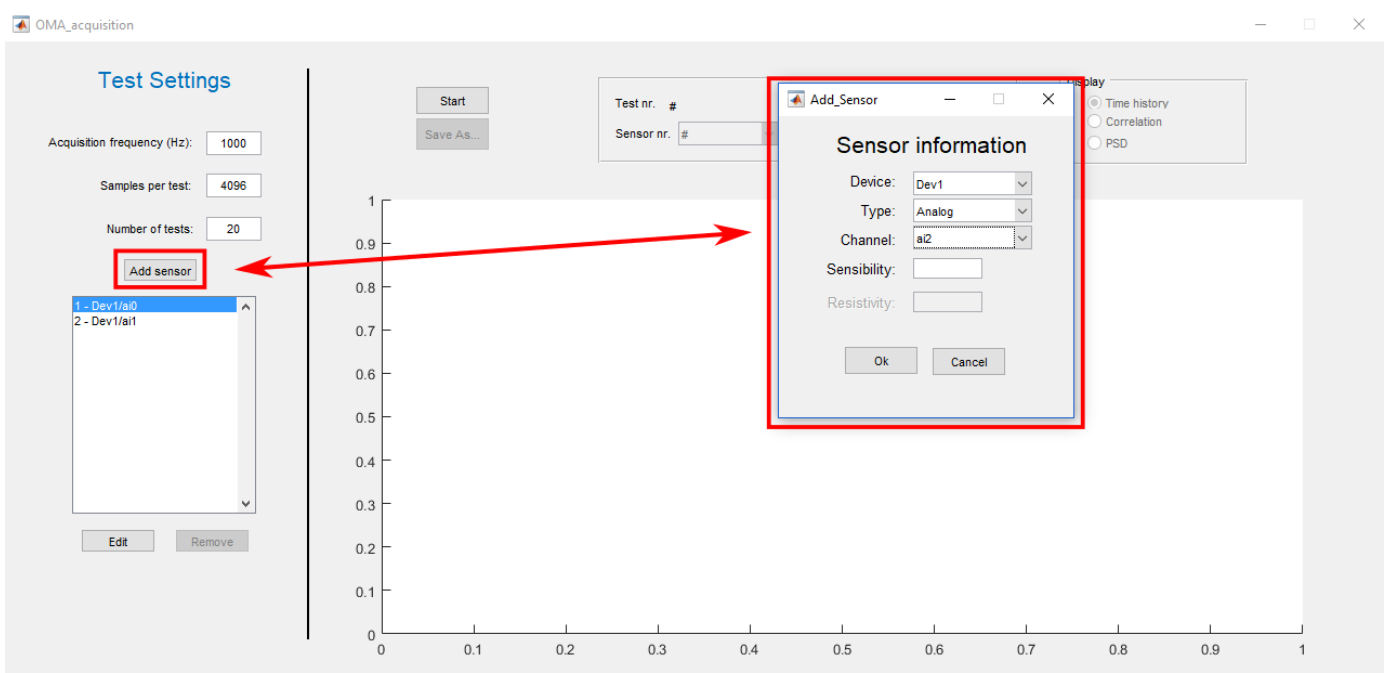

Figura A.3: Adição dos sensores utilizados no teste. 
3. Uma vez que todos os sensores estão listados, o teste pode ser inicializado clicando em "Start".

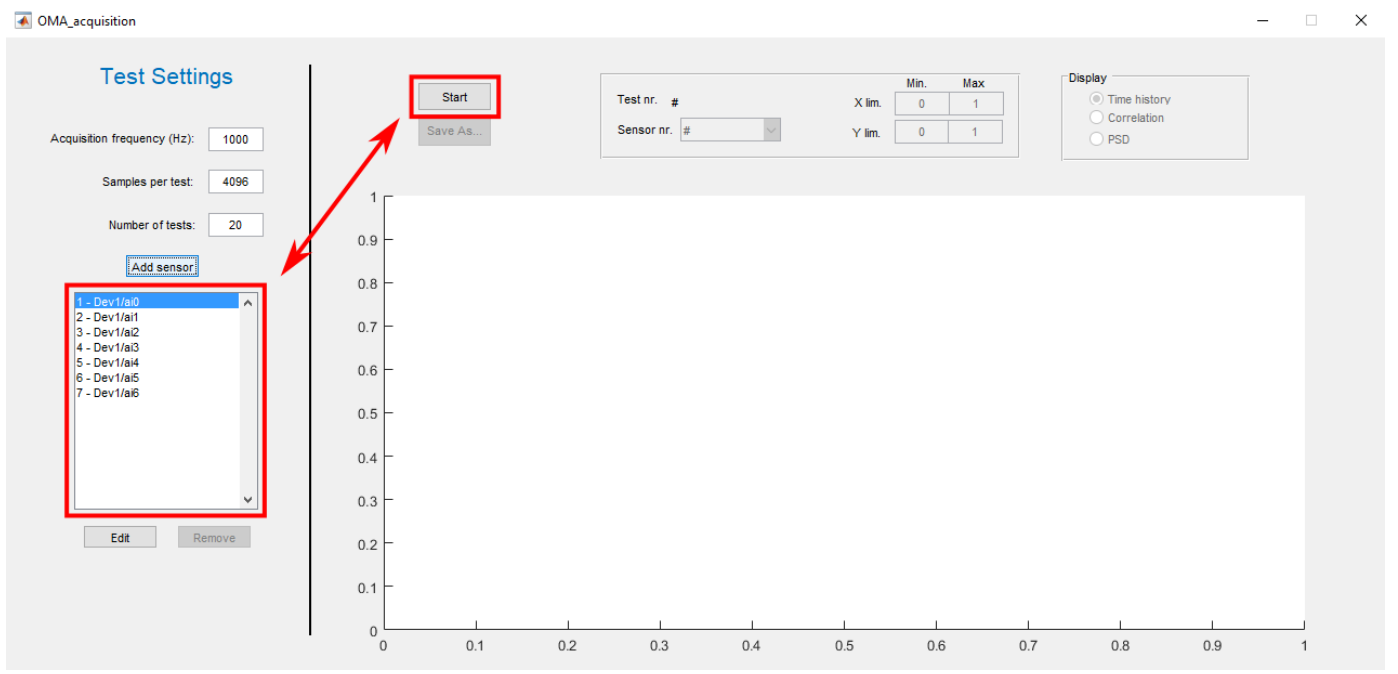

Figura A.4: Início da aquisição dos dados.

4. Ao completar a quantidade de amostras de um teste (definido na etapa 1), os resultados para cada sensor podem ser observados de diferente formas: no tempo, na forma de funções de correlações e na forma de densidades espectrais de potência. Nas funções de correlação e densidades espectrais, os cálculos são realizados utilizando os testes anteriores (fazendo médias) enquanto no gráfico das amostras no tempo apenas o atual teste é utilizado.

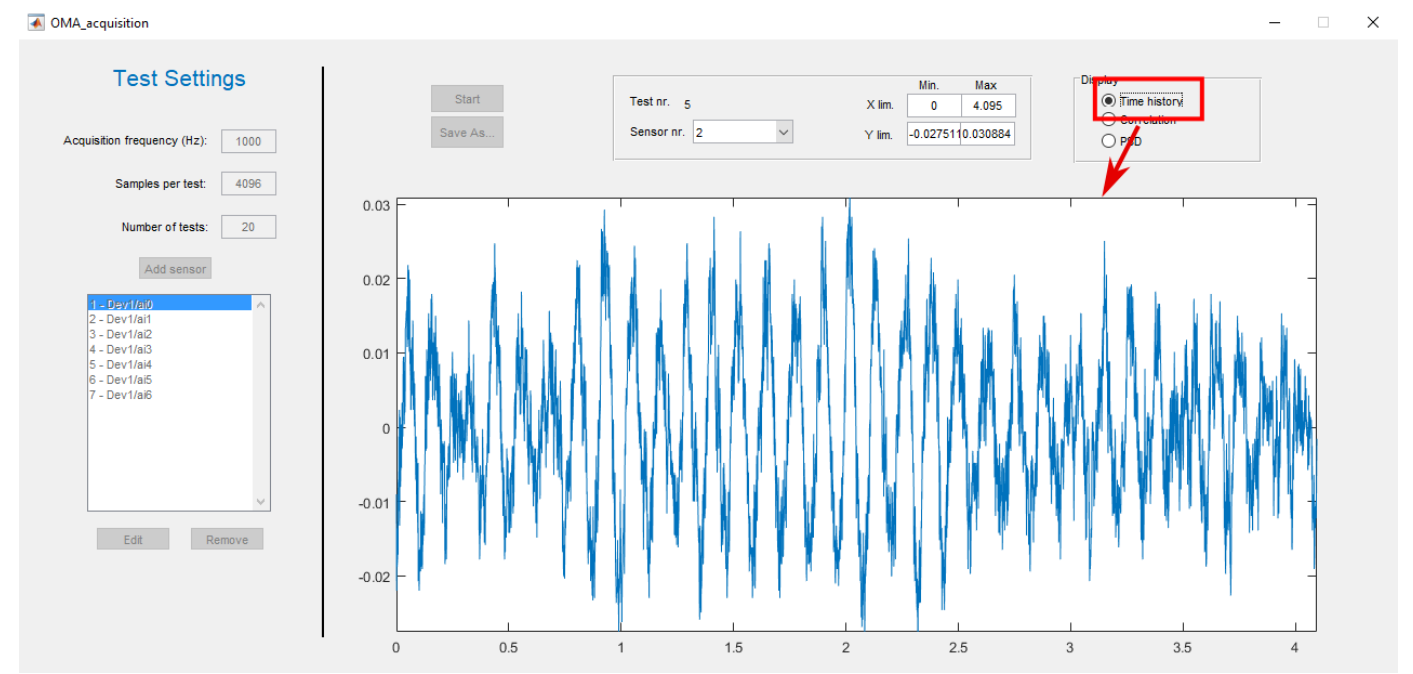

Figura A.5: Gráfico das amostras obtidas no tempo. 


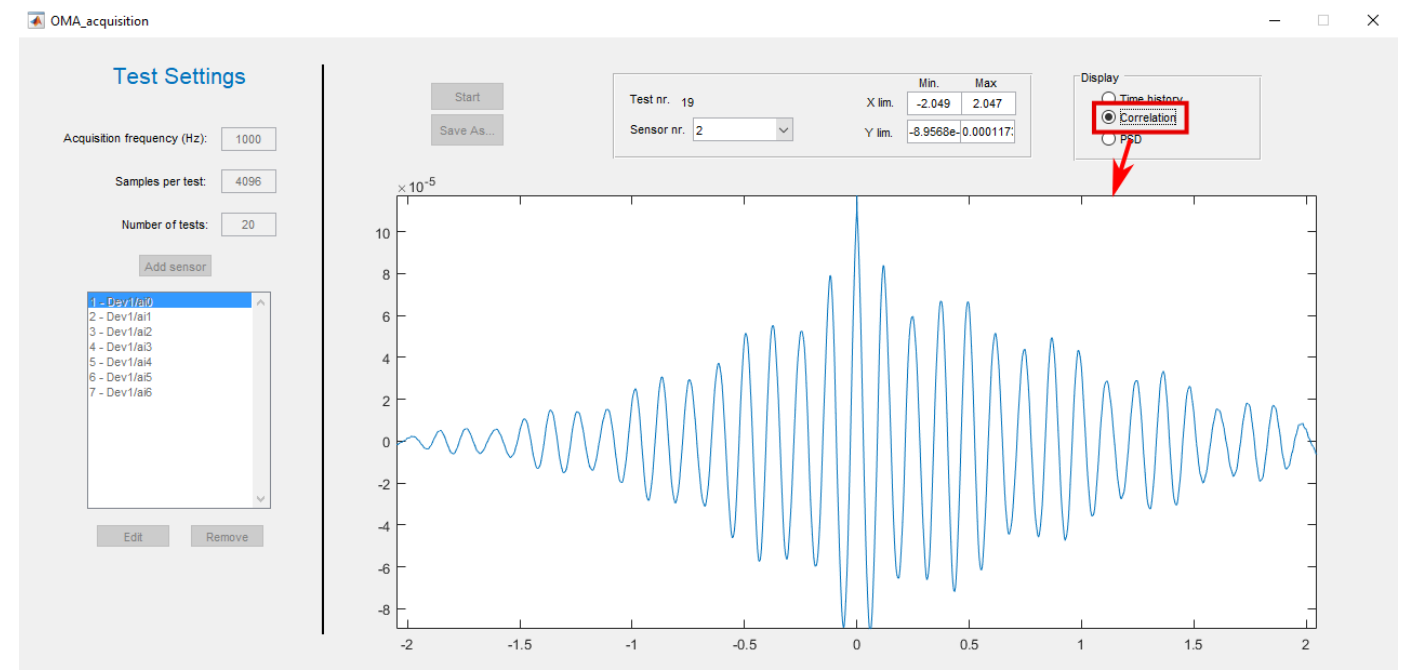

Figura A.6: Função de correlação das amostras coletadas.

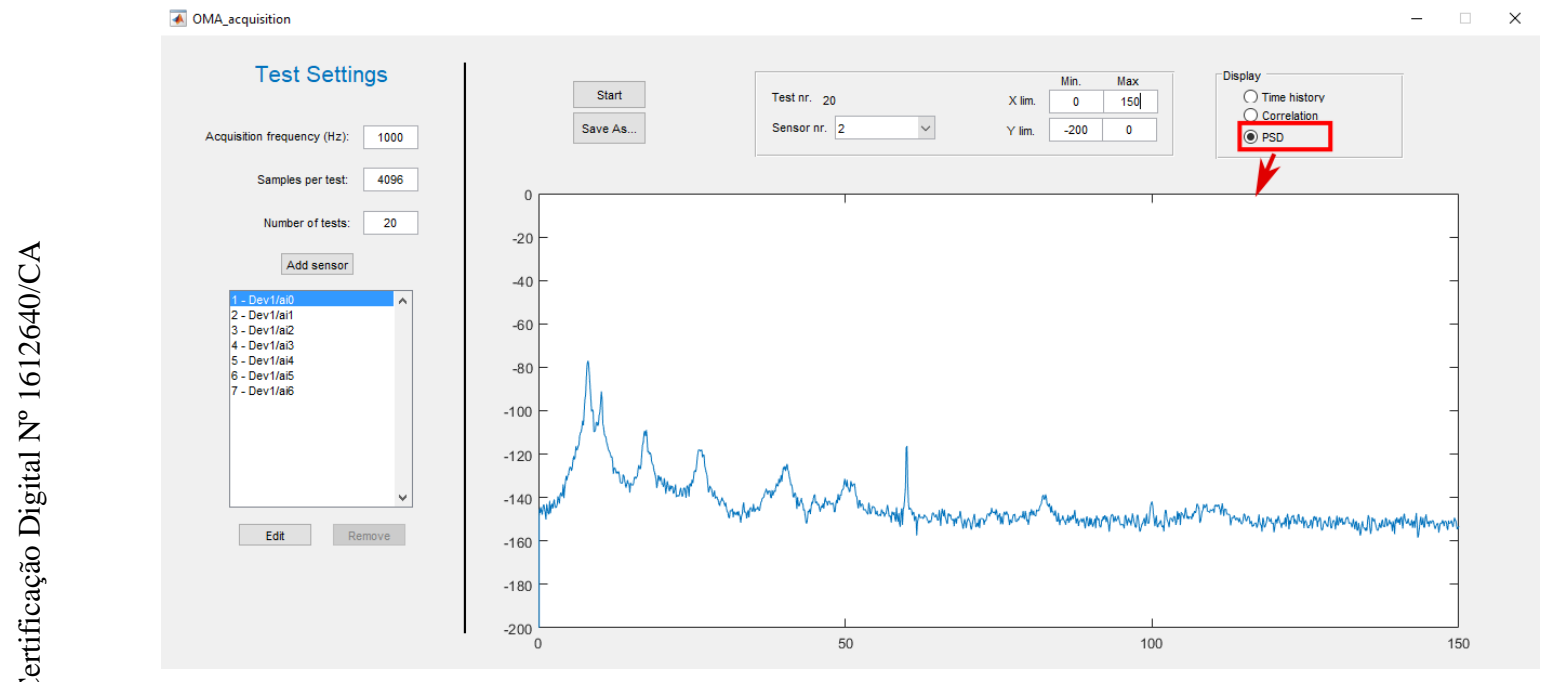

Figura A.7: Densidade espectral de potência das amostras coletadas.

5. Ao acabar todos os testes o botão "Save"é habilitado, permitindo que as amostras e suas estatísticas sejam salvas em formato ".mat". 


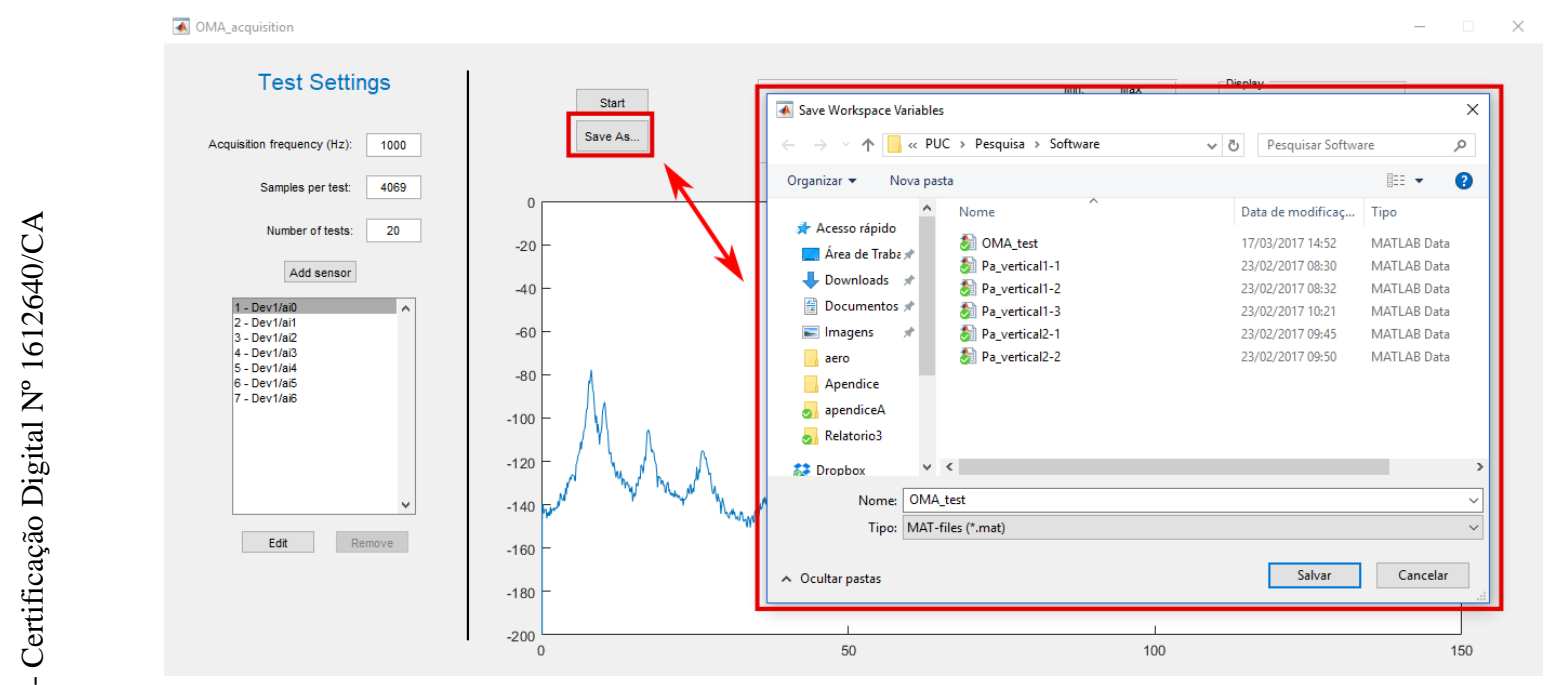

Figura A.8: Salvamento das amostras coletadas durante os testes. 
B

Artigos produzidos

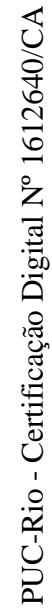


DINAME 2017 - Proceedings of the XVII International Symposium on Dynamic Problems of Mechanics A. T. Fleury, D. A. Rade, P. R. G. Kurka (Editors), ABCM, São Sebastião, SP, Brazil, March 5-10, 2017

\title{
Operational modal analysis under wind load using stochastic sub- space identification
}

\author{
Gustavo B. Wagner ${ }^{1}$, Damien Foiny ${ }^{1}$, Rubens Sampaio ${ }^{1}$, Roberta Lima ${ }^{1}$ \\ ${ }^{1}$ Pontifícia Universidade Católica do Rio de Janeiro - Rua Marquês de São Vicente, 225, Gávea - Rio de Janeiro, RJ \\ - Brasil - 22451-900 , gustavo_gbw@hotmail.com, damien.foiny@gmail.com, rsampaio@puc-rio.br, robertalima@puc- \\ rio.br
}

Abstract: The extraction of modal parameters from a real structure represents an important step in modal analysis. When only the output signal is available in an experiment, the system identification process is referred as operation modal analysis (OMA). Applications of those cases are fond for structures where the ambient excitation (wind, traffic, waves, nearby systems, etc.) can not be removed or is the only possible one. Since the input signals can not be measured, some assumptions in their random nature are needed together with a stochastic modeling of the system. Among several methods, the stochastic subspace identification (SSI) has shown to be a consistent one and, therefore, was chosen to be used in this paper. Here, the modal analysis of a system under wind load is studied. The fluid-structure interaction force is usually not easy to be represented and its whiteness (assumption made in most of OMA methods) can not be easily conformed. Therefore, a two floor building model is used for an experimental validation, where different fluidstructure interactions were created. The paper begins with a presentation of the discrete state space model followed by the SSI theory. Two popular SSI algorithms are presented: covariance-driven and data-driven. A efficient way to correctly select the modal parameters is discussed together with a procedure to analyze the results. To exemplify the identification process, experimental results are shown and the identified parameters are listed. As conclusion, the wind has shown to be a good excitation source for OMA once the system has been correctly identified.

Keywords: Operational modal analysis, System identification, Stochastic subspace methods, wind excitation, experimental validation

\section{INTRODUCTION}

Operational Modal Analysis (OMA) consist in finding the dynamic characteristic of a structure through its modal parameters using output-only signals. Differently from the classical approach of Experimental Modal Analysis (EMA), where the input signal are also measured, OMA only uses the stochastic nature of the inputs, assumed to be random due the ambient conditions. This fact allows system identification to be done under circumstances where EMA is limited, which includes: large and heavy structures, where a controlled input is hard to apply and expensive, and identification of systems under operational conditions, where interferences from the location can not be eliminated.

With its majors developments happening in the early 1990s, applications of OMA in the structural dynamic field is far from reaching its total potential. Nowadays, OMA has been used as tool in two main areas. The first is in the model validation of large structures such as bridges, tall buildings, stadiums and oil rigs (Rainieri and Fabbrocino, 2014)(Rodrigues, 2004)(Brincker and Ventura, 2015). Those structures have in common the heavy weight and an acting ambient forces. The excitation sources can be the wind, traffic, and waves which are difficult to model and measure. Therefore, OMA methods for parameters estimations suits very well in those cases (Reynders et al., 2016) (Reynders et al.,2008a). Recent articles have also focused on the variance estimation of the modal parameters. Mellinger et al. (2016), for example, measured the uncertainties in the modal parameter of an aircraft during in-flight tests. OMA has also been developed in the recent years for the field of structural health monitoring (SHM)(Liu, 2011)(Farrar and Worden, 2013)(Deraemacker and Worden, 2010). SHM is done by a periodic modal identification, which evaluates a possible change in the modal parameters. Cracks, corrosion, unfastened bolts, etc. usually reduces the system stiffness modifying natural frequencies and mode shapes.

The purpose of this article is to demonstrate a complete procedure for system identification of a real structure under wind load using stochastic subspace method. Becoming popular in the 2000s, stochastic subspace identification (SSI) consists in a collection of techniques that can be formulated in a consistent framework, where properties of the system can be estimated through a matrix subspaces. The two principal subspace algorithms found in the literature are the covariance-driven and the data-driven, which consist in estimating the system controllability matrix using covariance matrices or orthogonal projections of the output signals. For a clear understanding of such methods, the extension of the state space model are done by arranging the data in Hankel matrices. The method performance heavily depends in the Hankel matrices dimensions, and therefore are parameters that need to be carefully chosen. 
DINAME 2017 - Proceedings of the XVII International Symposium on Dynamic Problems of Mechanics A. T. Fleury, D. A. Rade, P. R. G. Kurka (Editors), ABCM, São Sebastião, SP, Brazil, March 5-10, 2017

DINAME 2017

\title{
Dynamical Systems Identification with Smooth Decomposition
}

\author{
Damien Foiny ${ }^{1}$, Gustavo B. Wagner ${ }^{1}$, Rubens Sampaio ${ }^{1}$, Roberta Lima ${ }^{1}$ \\ 1 Pontifical Catholic University of Rio de Janeiro (PUC-Rio), Department of Mechanical Engineering, Vibrations \\ and Dinamics Laboratory, Rua Marquês de São Vicente, 225, Gávea - 22453-900, Rio de Janeiro - RJ, Brazil,
} damien.foiny@gmail.com, gustavo_gbw@hotmail.com, rsampaio@puc-rio.br, robertalima@puc-rio.br

Abstract: Smooth Decomposition (SD) is a multivariate data or statistical analysis method to find normal modes and natural frequencies in an spatial data field. The projection used for this method is made such as it keeps the maximum variance possible for the displacement vector and also as it keeps the smoothest motions along time. From this method we can get the "energy" participation in the response of each normal mode during the simulation or the experimental test which can be a relevant information to validate results concerning the identification process. This method of identification can be used for linear and nonlinear systems and uses only output data given that the excitation satisfies some properties normally met by a well chosen random excitation, as a white noise, for example. The objective of this method is to identify systems from their displacement field under ambient excitation which, in many cases, can be hard to compute or to describe. As the method is only based on the covariance matrices of the displacement field and the corresponding velocity field, it is no needed further considerations and approximations. In this point the method is a great tool for modal analysis and system identification. In this paper, the presentation of the method is firstly done which will show us how we can interpret the results of SD for different systems and then the application of SD on simulated multi-DoF damped and undamped systems is performed and discussed to understand how SD can be a great tool for modal analysis. A discussion about the quality of the excitation is also performed.

Keywords: Smooth Decomposition (SD), System Identification, Operational Modal Analysis (OMA), Nonlinear Parameters Identification

\section{INTRODUCTION}

The Smooth Decomposition (SD) is a statistical analysis technique for finding structures in an ensemble of spatially distributed data such that the vector displacement not only keeps the maximum possible variance but also the motion, as the velocity field, is as smooth in time as possible. Closely related with the SD are the dual smooth modes used in the framework of oblique projection to expand a random response of a system. The concept of dual mode with the associated decomposition defines a tool that transforms the SD in an efficient modal analysis tool. This method of identification can be used for linear and nonlinear systems and uses only output data as soon as the excitation satisfies some properties normally met by a well chosen random excitation, as a white noise, for example.

The main properties of the SD are discussed and some optimality characteristics of the expansion are deduced. The parameters of the SD (using the dual smooth modes and the smooth values) give access to a modal parameters of a linear system in terms of mode shapes, resonance frequencies and modal energy participations. This part is a remarkable improvement with respect to the standard modal analysis methods. This novel modal analysis of a linear system is illustrated by examples.

One of the examples, to show the main features of the method, is a simple multi-DoF undamped system subject to a random excitation that is identified from the output signal. Then, more complex examples of a multi-DoF system are identified. A discussion concerning the difficulty to identify systems with high damping coefficient is made. We also study a case which can be a first step before considering continuous systems with the partially observed case. Finally we will discuss about the importance of the excitation quality for such a method.

It is interesting to say that this is a new method, not yet compared with the methods known in the literature as Operational Modal Analysis (OMA). So far the only association between SD and OMA is the fact that both methods use output signals for the identification and they require random excitation. However the theories are different. SD is a type of Karhunen-Loève Decomposition, using correlations and projections in the modes whereas OMA uses the controllability matrix and correlations of the measured signals that are not necessarily the state of the system.

\section{DESCRIPTION OF THE SMOOTH DECOMPOSITION METHOD}

First we will present the basis of this method and its main objective. There is already another well known method called the "Karhunen-Loève Decomposition (KLD)" or the "Proper Orthogonal Decomposition (POD)" used to analyze random data. This method is not presented in this article. The main objective of KLD or POD consists in finding the base 


\title{
THE ROBUST SMOOTH ORTHOGONAL DECOMPOSITION METHOD FOR OPERATIONAL MODAL ANALYSIS
}

\author{
G.B. Wagner ${ }^{1}$, D. Foiny ${ }^{2}$, R. Lima $^{3}$, R. Sampaio ${ }^{4}$ \\ ${ }^{1}$ Master’s student, Pontifícia Universidade Católica do Rio de Janeiro, Brazil, gustavo.wagner@aluno.puc-rio.br \\ ${ }^{2}$ Master's student, Pontifícia Universidade Católica do Rio de Janeiro, Brazil, damien.foiny@gmail.com \\ ${ }^{3}$ Prof. , Pontifícia Universidade Católica do Rio de Janeiro, Brazil, robertalima@puc-rio.br \\ ${ }^{4}$ Prof. , Pontifícia Universidade Católica do Rio de Janeiro, Brazil, rsampaio@ puc-rio.br
}

\begin{abstract}
The smooth orthogonal decomposition (SOD) method has been studied in the last past years as an outputonly modal parameters identification technique for linear normal modes and natural frequencies extraction. Seen as a variant of the proper orthogonal decomposition (POD), the SOD method consists in the identification of a projection base that not just maintain the maximum variance of a scalar field, but also perform it in the smoothest possible way. In this paper a new implementation of the method is proposed to overcame its noise sensitivity problem. This new implementation also allows the modal parameters uncertainties to be quantified. With a numerical simulation and an experimental test, the method's performance is demonstrated and validated.
\end{abstract}

Keywords: smooth orthogonal decomposition, system identification, noise control

\section{INTRODUCTION}

In operational modal analysis (OMA), orthogonal decomposition methods are a recent family of identification techniques based on a multivariate statistics method. They all have been developed as extension of the proper orthogonal decomposition (POD) to overcome some of its limitations when applied to modal analysis. For linear structures, POD requires a priori knowledge of the system's inertial matrix to relate the proper orthogonal modes (POMs) to the linear normal modes (LNMs), as presented in [1][2][3]. Another inherent limitation consists in the fact that the proper orthogonal values (POVs) contains only informations about the energy of the POMs in the acquired data, and therefore are not uniquely related to them. Those disadvantages were first overcome by the smooth orthogonal decomposition method (SOD) in [4] and after by the state-variable modal decomposition method (SVMD) in [5][6].

The smooth orthogonal decomposition (SOD), also known as smooth Karhunen-Loève decomposition [7], consists in the identification of a projection base that not just maintain the maximum variance of a scalar field (in case of modal analysis, the structure displacement, velocity or acceleration), but also 
Noname manuscript No.

(will be inserted by the editor)

\title{
Operational modal analysis of a wind turbine blade under wind load using stochastic subspace identification
}

\author{
Gustavo B. Wagner · Damien Foiny · Pablo \\ Milheiro - Rubens Sampaio
}

Received: date / Accepted: date

\begin{abstract}
System identification is an important step when performing modal analysis. When dealing with structures where only the response signal are measured, the identification technique is addressed in the literature as operational modal analysis (OMA). Applications of those cases are found for structures where the ambient excitation (wind, traffic, waves, nearby systems, etc.) can not be removed or is the only possible one. Since the input signals can not be measured, some conditions in their random nature must be fulfilled together with a stochastic modeling of the system. In this paper, the modal parameters of a small wind turbine are extracted using the stochastic subspace identification (SSI) method which became popular thanks to its consistency and robustness. Two of the most popular SSI algorithms, covariance-driven and data-driven, are presented. The wind was chosen to be the random excitation and it allowed the modal identification to be performed in this paper prescribed frequency band. A procedure to analyze and validate the experimental results is also given.
\end{abstract}

Keywords Operational modal analysis $\cdot$ Stochastic subspace $\cdot$ Wind excitation $\cdot$ System identification · Wind turbine blade

\section{Introduction}

Operational Modal Analysis (OMA) consist in finding the dynamic characteristics of a structure through its modal parameters using output-only signals. Differently from the classical approach of Experimental Modal Analysis (EMA), where the input signals are also measured, OMA only uses the stochastic nature of the inputs, assumed to be random and excite all the modes in the prescribing frequency band. This fact allows system identification to be done under circumstances where EMA does not work properly, which includes: large and heavy structures, where a controlled input is hard to get and expensive, and identification of systems under operational conditions, where interferences from the ambient can not be eliminated.

G. B. Wagner

PUC-Rio, Rua Marquês de São Vicente, 225, Gávea, Rio de Janeiro, Brazil

Tel.: +55-21-XXXXXXXX

E-mail: gustavo.wagner@aluno.puc-rio.br 
Noname manuscript No.

(will be inserted by the editor)

\title{
Implementation of sliding filters in the smooth orthogonal decomposition
}

\author{
Gustavo Wagner · Damien Foiny · Roberta Lima \\ Rubens Sampaio
}

Received: date / Accepted: date

\begin{abstract}
In the last past years, the Smooth Orthogonal Decomposition (SOD) has been studied as an output-only modal analysis technique. It consists in a multivariate statistics method that has been formulated as an extension of the Proper Orthogonal Decomposition by adding a constraint on the decomposition's smoothness. The benefits of this extension are: the possibility of a direct estimation of the system's natural frequencies and the identification of the normal modes without the necessity of a priory knowledge of the mass matrix. The SOD's projection basis is defined as the basis that maintains the maximum variance of a scalar field and also performs it in the smoothest possible way in relation to time. With the formulation developed by this definition, the amount of identified modes with this method is restricted by the number of system's measured responses. Another characteristic of this method is the fact that the identification of the modal parameters is always performed in an ascending order in relation to the natural frequencies. This means that the modes with higher natural frequencies can only be identified after the lower ones. Therefore, the identification of high frequency modes is only possible when the amount of measured responses exceeds the number of lower frequency modes. This limitation is overcome in this paper by presenting a new way to implement the SOD with the addition of a sliding band pass filter. This new process also allows a stabilization diagram to be constructed.
\end{abstract}

Keywords Smooth Orthogonal Decomposition · Operational Modal Analysis · System Identification · Sliding Filter $\cdot$ Stabilization Diagram

\section{Introduction}

In operational modal analysis (OMA), orthogonal decomposition methods are a recent family of identification techniques based on a multivariate statistics method. They all have been developed as extensions of the Proper Orthogonal Decomposition (POD) to overcome some of its limitations when applied to modal analysis [1-4]. The Smooth Orthogonal Decomposition (SOD), also known as smooth Karhunen-Loève Decomposition [5], is a method that

G. B. Wagner

PUC-Rio, Rua Marquês de São Vicente, 225, Gávea, Rio de Janeiro, Brazil

Tel.: +55-21-XXXXXXXX

E-mail: gustavo.wagner@aluno.puc-rio.br 


\title{
Dynamical System Identification and Modal Analysis using Smooth Decomposition
}

\author{
Damien Foiny ${ }^{1}$, Gustavo B. Wagner ${ }^{1}$, Roberta Lima ${ }^{2}$, Rubens Sampaio ${ }^{2, *}$ \\ Pontifical Catholic University of Rio de Janeiro (PUC-Rio) \\ Department of Mechanical Engineering \\ Vibrations and Dynamics Laboratory \\ Rua Marquês de São Vicente, 225, Gávea - 22453-900, Rio de Janeiro - RJ, Brazil
}

\begin{abstract}
Smooth Decomposition (SD) is a multivariate data or statistical analysis method to find normal modes, natural frequencies and energy partition in a spatial data field. The projection used for this method is chosen to keep the maximum variance possible for the generalized displacement vector and also to keep the smoothest motions along time. This method gives the "energy" participation of each normal mode in the response during the simulation or the experimental test which is a crucial information for identification of non-linear systems. This method of identification can be used for linear and nonlinear systems (since we do have access to the energy partition) and uses only output data provide the excitation satisfies some properties normally met by a well chosen random excitation, as a white noise, for example. The objective of this method is to identify systems from their generalized displacement field under ambient excitation which, in many cases, can be hard to compute or to describe. As the method is only based on the covariance matrices of the generalized displacement field and the corresponding velocity field, no further considerations and approximations are needed. Due to this feature, the method is a great tool for modal analysis and system identification. In this paper, the presentation of the
\end{abstract}

\footnotetext{
*Corresponding author. Postal address defined before. Tel.: +55 (21) 3527-1172

Email address: rsampaio@puc-rio.br (Rubens Sampaio)

${ }^{1}$ Master's student, Pontifical Catholic University of Rio de Janeiro, Brazil

${ }^{2}$ Professor, Pontifical Catholic University of Rio de Janeiro, Brazil
} 